\title{
THE VELOCITY STRUCTURE WITHIN THE SUBDUCTED SLAB BELOW THE LOWER NORTH ISLAND, NEW ZEALAND
}

\author{
Pauline Maria Galea
}
A thesis presented in fulfilment of the requirements for the degree of
Doctor of Philosophy
in
Geophysics

at

Victoria University of Wellington

Institute of Geophysics

Research School of Earth Sciences

Victoria University of Wellington

Wellington, New Zealand

1994 
To

the memory of

Professor Jim Ansell

who left this world

and to

Clara

who was born into it

both during the last year of this study 


\begin{abstract}
A shallow aftershock sequence in the Hawkes Bay region of the North Island, New Zealand (May 1990) was recorded with high quality on an L-shaped, 7-station array of 3-component, short-period seismographs at Wellington, such that the seismic waves travelled almost along strike of the subducted Pacific plate in this region. The arrival times at the stations of the $P_{n}$ wave pulse from a number of aftershocks could be picked sufficiently accurately for a least-squares inversion to be carried out for wavefront speed, $c$, and incident azimuth, $\phi$. The results show a high apparent velocity, $8.7 \pm 0.2 \mathrm{~km} / \mathrm{s}$, and an azimuth which is shifted by $6.0 \pm 2.5^{\circ}$ east of the true epicentre - station azimuth.

The azimuthal anomaly, $\delta \phi$, has been interpreted as due to lateral refraction of $\mathrm{P}_{\mathrm{n}}$ off the subducted slab. The effect of different geometries of the slab on the $P_{n}$ wavefront characteristics ( $c$ and $\delta \phi$ ) at Wellington have been explored through both simple geometrical considerations (in the case of a plane or cylindrical slab) as well as through 3-dimensional ray tracing (in the case of irregular curvature of the slab). It has been shown that a plane or cylindrical slab would require P-wave velocities of about $9.0 \mathrm{~km} / \mathrm{s}$ to exist within it in order to fit both $c$ and $\delta \phi$, whereas a model of the slab which departs from a regular cylinder and has a small updip component along strike can fit the observations with P-wave velocities of $8.75 \mathrm{~km} / \mathrm{s}$ in the high velocity medium. This model has been proposed by Ansell and Bannister (1991) after detailed consideration of the shallow seismicity that defines the slab surface in the lower North Island.

Information about the nature of the high velocity medium has been obtained by modelling the waveforms through generation of synthetic seismograms by the reflectivity technique of Kennett (1983). The large number of aftershocks within a small source region, and the sampling of much the same wavepath, meant that a sufficient number of seismograms had very similar and characteristic features that could be modelled. The typical seismogram of the data set had a simple $P_{n}$ wavepulse, followed immediately by a complex, high frequency (up to $15 \mathrm{~Hz}$ ) phase (here referred to as $\mathrm{P}_{\mathrm{hf}}$ ) and a high amplitude, lower frequency phase that dominated the $\mathrm{P}$ wavetrain (here referred to as $\bar{P}$ ). A velocity profile that contained a layer of $8.75 \mathrm{~km} / \mathrm{s}$ material at least $4 \mathrm{~km}$ thick, underlying "normal" mantle material of P-velocity $8.2 \pm 0.2 \mathrm{~km} / \mathrm{s}$, and whose surface lies approximately $18 \mathrm{~km}$ below the slab surface reproduced the observed seismogram features well. The presence of velocity gradients above and below the layer is not excluded. A gradual decrease in velocity below the layer in fact gives a better fit of the $P_{n}$ pulse shape. By breaking down the synthetic seismogram into simpler versions, using Kennett's wavefield approximation technique, it has been shown that the $P_{n}$ wave propagates through the high velocity layer, the $\mathrm{P}_{\mathrm{hf}}$ phase through the overlying layers as a sequence of reflections and refractions, and the $\tilde{\mathrm{P}}$ group as a reverberatory phase in a crustal waveguide, with its energy mostly in the form of free surface reflections and $\mathrm{S}$ to $\mathrm{P}$ conversion. These results have also been confirmed by ray tracing.
\end{abstract}


Waveform modelling has also clearly shown that a low velocity layer (representing subducted sediment) on the top of the subducted slab produces a highly characteristic imprint on the synthetic seismogram, in the form of an energetic, reverberatory, lower frequency signal late in the P-wavetrain. Wavefield approximations show that this is also a crustal waveguide effect, with a strong component of mode conversion at the free surface, but $\mathrm{P}$ - S conversion appears to be the dominant mechanism. Seismograms very similar to such synthetic ones have been observed for the Weber aftershocks recorded at stations along the northern East Coast. The presence of such a low velocity layer in the East Coast region is thus implied, consistently with previous proposals.

The petrological implications of the high velocity layer in the subducted Pacific plate are discussed. The most likely explanation is that it represents the maximum $P$ velocity of an anisotropic layer within the Pacific upper mantle. It is proposed that the conditions of stress orientation, pressure and temperature at approximately $36-50 \mathrm{~km}$ depth in this region induces a strong realignment of olivine crystals with their fast direction along strike of the slab, normal to the maximum compressive stress axis. The upper mantle of the segment of the Pacific ocean just east of the Tonga - Kermadec trench and the North Island has been shown in this study to possess $\mathrm{P}$-wave anisotropy, with the P-velocity reaching a maximum of $8.37 \mathrm{~km} / \mathrm{s}$ in a direction $\mathrm{N} 60^{\circ} \mathrm{E}$. This result was obtained by analysing a large set of ISC travel times from earthquakes along the Tonga - Kermadec - New Zealand subduction zone recorded at stations Niue, Rarotonga and the Chatham Islands. It is suggested that an enhancement of this anisotropy, accompanied by some re-orientation, takes place as the upper mantle medium is subjected to the new stress conditions in the initial stages of subduction. 


\section{CONTENTS}

Abstract

Acknowledgements

Figures

Tables

Chapter 1: Geological/Tectonic Setting - Introduction

1.1 New Zealand and the South West Pacific 1

1.2 The Tectonics and Seismicity of New Zealand .............................................

1.3 Velocity Models for New Zealand ...................................................................

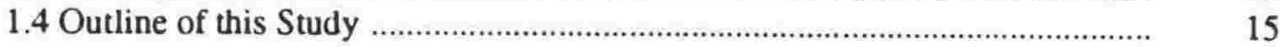

Chapter 2: Array Measurement of Apparent Velocities along strike of the Subducted Slab

2.1 Introduction 17

2.2 The L-Network 17

2.3 The Data Sets used 19

2.4 Inversion of Arrival Times for Wavefront Velocity ..........................................

2.5 Results

2.6 Travel Time/Distance Graph

2.7 Particle Motions

Chapter 3: The Effects of Slab Geometry on the Speed and Azimuth of a Refracted Wavefront

3.1 Introduction 45

3.2 A Plane Dipping Refractor

3.3 A Cylindrically Curved Refractor

3.4 3-Dimensional Ray Tracing

\section{Chapter 4: The Reflectivity Method}

4.1 Introduction

4.2 Matrix Representation of Plane Waves ....................................................... 65

4.3 The Stress-Displacement Propagator Matrix P ................................................. 69

4.4 The Wave Propagator ...................................................................................

4.5 Reflection and Transmission Matrices ......................................................... 72

4.6 A Stack of Uniform Layers ........................................................................... 77

4.7 The Response of a Medium to a Source .......................................................... 79

4.8 The Surface Displacement ........................................................................... 81

4.9 Recovery of the Time-Domain Response ..................................................... 83

4.10 Allowance for Attenuation ...................................................................... 85

4.11 Practical Application of the Reflectivity Method ....................................... 86 


\section{Chapter 5: Waveform Modelling}

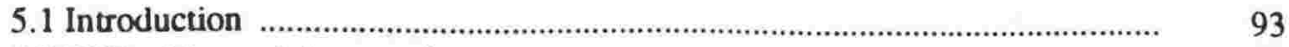

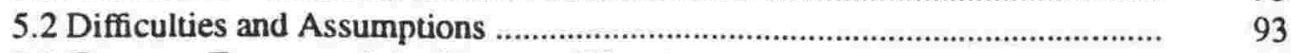

5.3 Common Features of the Observed Waveforms ............................................ 95

5.4 Basic Velocity/Attenuation Model ............................................................. 98

5.5 Features and Explanation of the Synthetic Seismogram ................................. 102

5.6 Modelling Velocity Features in the Subducted Upper Mantle ...................... 111

5.7 The Effect of a Low-Velocity Layer at the top of the Subducted Slab ............. 115

5.8 The Effects of other Parameters on the Synthetic Seismogram ..................... 119

5.9 Comparison with 3-dimensional Ray-Tracing Results .................................. 126

5.10 Conclusion ............................................................................................ 127

Chapter 6: Upper Mantle Anisotropy in the South West Pacific Ocean

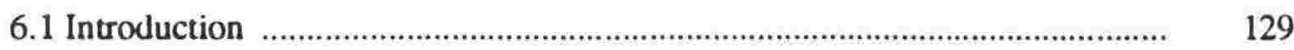

6.2 Data …….................................................................................... 130

6.3 Azimuthal Dependence of P-residuals and P-velocities ............................... 135

6.4 Implications and Discussion ..................................................................... 141

Chapter 7: Summary and Discussion of Results

7.1 Main Results ............................................................................................... 143

7.2 Petrological Implications of the High Velocities ............................................... 144

7.3 Discussion of the Velocity Profile ............................................................... 154

7.4 Unanswered Questions and Suggestions for Further Research ..................... 155

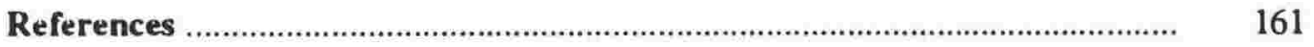

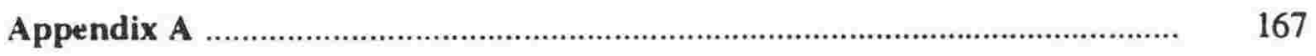

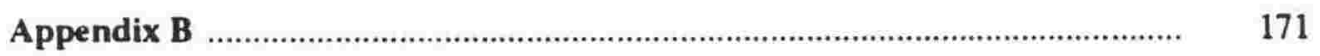

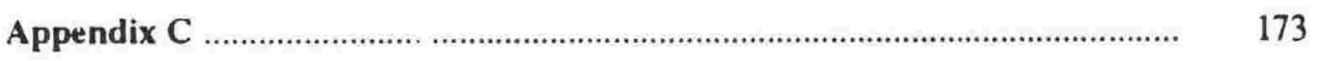

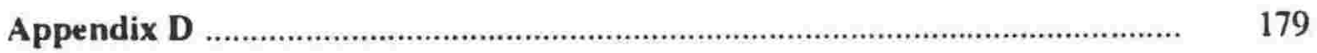

Published Papers I, II ......................................................................... Inside. Back Cover 


\section{ACKNOWLEDGEMENTS}

The years leading up to this thesis have brought me in contact with a number of wonderful people, each of who has helped in some way or another toward making this work possible.

Sadly, the foremost person I wish to thank is no longer here to read this - my supervisor, Professor James H. Ansell. I am glad of this opportunity to express my deep respect and affection for Jim, who was an inspiration throughout. His keen sense of understanding in all fields - academic and human - helped me through all the difficult patches, and made my stay at Victoria University all the more pleasant and significant. Thank you, Jim.

Dr. Tim Stern took over as my supervisor after Jim passed away - I am grateful for his assistance and valued comments on my thesis. Dr. John Taber had the task of re-reading the thesis. I greatly appreciated his help and much valued advice, knowing that he had to work under difficult circumstances.

All staff and students who frequented the Data Processing Laboratory and Institute of Geophysics gave their help at one time or another - Wayne Richardson, Allister Gorman, Mark Chadwick, John Taber, Ken Gledhill, Jennifer Rollo, Luo Xun, Graham Mckenzie and others. These and other staff members provided useful discussions. I am especially grateful to Allister Gorman and John Taber, who continued to send their help through e-mail and other means when I had to finish my thesis in Malta.

This Ph.D. was carried out under the auspices of a Commonwealth scolarship provided by the New Zealand Government. For this I am very grateful. I also wish to thank the New Zealand Vice Chancellor's Committee, which administers the scholarships, in particular Ms. Cathe Carpenter, as well as Ms. Maureen Penning, Scholarships Officer at Victoria. These ladies were always extremely understanding and ready to help. This was especially appreciated when I had to finish my thesis under several difficult circumstances. Without their help, it would not have been possible.

The staff at the Institute of Geological and Nuclear Sciences (formerly Geophysics Division of the Department of Scientific and Industrial Research (D.S.I.R.)) are thanked for their cooperation in providing data from National Network stations, and for useful discussions.

The final stages of writing-up of the thesis were carried out in Malta. I thank the Department of Information Science of the University of Malta for allowing me full use of their computer facilities, and the Faculty of Science for practical assistance.

My husband Joe deserves special thanks for actually being with me in New Zealand, and for his constant practical and moral support, and his patience, especially in the final year. His help in getting programs to run on new machines was especially appreciated. My parents and parents-in-law provided long-distance encouragement (and many prayers) from the other side of the world, and also much practical help during the final stages in Malta. Our little daughter Clara also helped by being a such a good baby through it all!

Finally, Joe and I would like to thank the many friends and acquaintances we made in New Zealand for their warmth and hospitality. They helped make this beautiful country an even better place for us to spend these past few years. 


\section{FIGURES}

1.1 Geographical setting of New Zealand

1.2 The Pacific/Indo-Australian plate boundary in New Zealand

1.3 Epicentres of New Zealand earthquakes deeper than $33 \mathrm{~km}$ in 1986

1.4 Epicentres of New Zealand earthquakes shallower than $33 \mathrm{~km}$ in 1986

1.5 National Digital Seismic Network of New Zealand

1.6 Deformation patterns in the subducted plate (Reyners, 1979)

1.7 Velocity models for the lower North Island

1.8 Upper mantle P and S velocities in New Zealand (Haines, 1979)

2.1 L-network. Wellington network and aftershocks of the 13 May 1990 Weber earthquake

2.2 Displacement response of an EARSS seismograph

2.3 Epicentres of Weber aftershocks - comparison of National Network locations and microearthquake network locations

2.4 Depth distribution of Weber aftershocks

2.5 3-component seismograms from Weber aftershocks

2.6 Array sections across L-network of some Weber aftershocks

2.7 Frequency - time analysis diagrams of some Weber aftershocks

2.8 Amplitude spectra of two time windows of a typical seismogram

2.9 Location of other events recorded on the L-network and used in this study

2.10 Seismograms from other events in the S. Hawkes Bay area and in the Lake Tennyson area

2.11 An example of the picking window in the xpick programme

2.12 A plane wavefront incident on an array of seismographs

2.13 First arrival travel time curve for North Island events recorded on KSE

2.14 Particle motion diagrams for some selected events

3.1 Geometrical setting for a refracted ray travelling long a plane dipping interface

3.2 Change of coordinate system at the point where a ray refracts off a dipping interface

3.3 Equal travel-time contours defining a wavefront at the earth's surface due to the wave path geometry of Figure 3.1

3.4 Variation of wavefront speed and azimuth with epicentre-station azimuth, and with slab dip

3.5 Geometry of a ray travelling along a cylindrically curved slab

3.6 Cylindrical model constructed for 3-d ray tracing

3.7 Cross-sections of 3-d velocity models

3.8 Modified cylindrical curvature model constructed for 3-d ray tracing following Ansell and Bannister (1991)

3.9 One velocity model that gives a good fit of both wavefront speed and azimuth 
4.1 Reflectivity of a stack of homogeneous layers

4.2 Reflection and transmission coefficients for incident $\mathrm{S}$ and $\mathrm{P}$ waves

4.3 Reflection and transmission matrices for a stratified region

4.4 Layering system used in recursive scheme (Kennett, 1983)

4.5 Response of a medium to a source

4.6 Illustration of the separation level $z_{J}$ (from Kennett (1988))

4.7 Contamination of the signal onset by "wraparound"

4.8 Real part of the integrand in the wavenumber integration at $6 \mathrm{~Hz}$

4.9 An arbitrary fault plane geometry

5.1 Some examples of vertical component seismograms showing similarity of features to be modelled

5.2 Two examples of record sections across the L-network, illustrating the $\bar{P}$ group

5.3 Data showing similarity of $P_{n}$ pulse shape

5.4 Existing P-velocity profiles for Wellington and Hawkes Bay areas

5.5 Layer version of basic velocity model MOD01

5.6 3-component synthetic seismogram generated by MOD01

5.7 Long - range synthetic profile generated by MOD01

5.8 Comparison of synthetic seismogram with some data

5.9 Ray-tracing through model MOD01

5.10 Wavefield approximations for synthetic seismogram from MOD01

5.11 Effect of low-velocity surface layer on synthetic seismogram

5.12 Effect of different velocity profiles in the region of the subducted upper mantle

5.13 Effect of depth of high-velocity medium

5.14 Effect of thickness of layer of high-velocity medium

5.15 Effect of velocity gradients bounding the high-velocity layer

5.16 Effect of low-velocity layer at top of subducted slab

5.17 Wavefield approximations for synthetic seismogram of Figure 5.16

5.18 Long-range profile for synthetic seismogram of Figure 5.16

5.19 A Weber aftershock recorded at East Coast stations PUZ, HBZ

5.20 Waipawa earthquake recorded at stations of the Hikurangi Margin refraction line

5.21 Effect of thickness of subducted low-velocity layer

5.22 Effect of P-velocity within the subducted low-velocity layer

5.23 Effect of source depth, for a source within the overlying crust

5.24 Effect of small changes in the fault plane parameters

5.25 Effect of velocity gradient in the crust

5.26 Effect of high-velocity layer at base of overlying crust

5.27 Effect of attenuation parameters in different regions of the lithosphere 
5.28 Synthetic sesimograms for a normal-faulting source at different depths in the subducted lithosphere

5.29 Cross-section of 3-d model

5.30 Final velocity profile

5.31 Final velocity profile, incorporating low-velocity subducted layer

6.1 Stations and selected Tonga - Kermadec - North Island events January 1970 - August 1987

6.2 P-residuals for all depths

6.3 Azimuth and latitude coverage by CIZ, NUE and RAR for path lengths shorter than $17^{\circ}$

6.4 Reduced travel-time plot for all path lengths, for shallow $(<100 \mathrm{~km})$ events

6.5 Variation of P-residual with propagation azimuth

6.6 Variation of P-velocity with azimuth

6.7 Dependence of measured P-velocity on source depth

6.8 Variation of mean P-velocity with azimuth, and least-squares fit sinusoid

6.9 As Figure 6.8, but events grouped into $0-50 \mathrm{~km}$ and $50-100 \mathrm{~km}$ depth ranges

7.1 Zonation of the oceanic lithosphere (Ringwood, 1982)

7.2 Layering of the ocean crust (Hsu, 1987)

7.3 Compressional wave velocities along crystallographic axes of olivine

7.4 Bending stress with depth below sea level within a subducting slab (Chapple and Forsyth 1979)

7.5 Orientation of olivine grains under axial compression (Wenk et al, 1991)

7.6 Example of shear wave splitting observed in the Weber data set

7.7 Creation of the Pacific plate $190 \mathrm{My}$ ago (Hilde et al, 1977)

7.8 Present day tectonics and magnetic lineations in the South Pacific (Molnar et al, 1975) 


\section{TABLES}

1.1 The New Zealand standard velocity model

2.1 L-network and Wellington network station data

2.2a The Wellington standard velocity model

2.2b The Hawkes Bay standard velocity model

2.3 Delay corrections applied to L-network stations and Wellington network stations

2.4a Array inversion results from the Weber data set, using the L-network

2.4b Array inversion results from the Weber data set, using the extended network

2.4c Array inversion results from S.Hawkes Bay data, using the L-network

2.4d Array inversion results from Lake Tennyson data set, using the L-network

3.1 P-velocities above and below a plane slab, of varying dip, that would produce observed values of $c$ and $\phi_{s}$

3.2 Wavefront characteristics at the surface due to refraction off a cylindrical surface

3.3 Construction of 3-d model used in CRT with modified cylindrical geometry

5.1 Starting velocity model MOD01 used in waveform modelling

5.2 Different attenuation models tested

A.1 Source parameters for the Weber data set

A.2 Source parameters for other central North Island events

A.3 Source parameters for Lake Tennyson data set 


\section{Chapter 1}

\section{Geological/Tectonic Setting \\ - Introduction}

\subsection{New Zealand and the South West Pacific}

The southwest corner of the Pacific Ocean, together with the smaller seas and land masses stretching to the east coast of Australia (Figure 1.1) is comprised of some interesting and intriguing geological domains. It is an area of complex tectonic history and active processes. The major tectonic process affecting the region at the present time is the interaction of the Pacific and Indo-Australian plates, which includes the subduction of the Pacific plate along a major portion of the length of the boundary zone. The plate boundary is characterised by the volcanism, shallow and deep seismicity and orogeny typical of such tectonic regions and delineated by a trench system composed of the Tonga, Kermadec and Hikurangi trenches. The North Island of New Zealand is situated on this plate boundary and therefore provides a suitable platform from which such processes may be closely observed.

The present ocean floors and continental masses making up the S.W. Pacific region are the result of a complex history of plate creation and destruction, movement, reorganization and deformation, which must be traced back to well before the break-up of Gondwana in the Jurassic. The process of unravelling this history is a fascinating challenge and has occupied numerous scientists for many decades. Using a diversity of geological and geophysical means, several reconstructions of the tectonic situation from the Jurassic to the present have been made $(e . g$ Christoffel and Falconer, 1972; Falconer, 1972; Larson and Chase,1972; Molnar et al,1975; Hilde et al,1977; Weissel et al,1977) yet many questions remain unresolved. Where clear magnetic lineations are preserved in the oceanic crust, such as on both sides of the Pacific-Antarctic ridge, the origin and movement of the oceanic plates can be traced back in time. Where such a magnetic signature is lacking, as in the case of oceanic crust formed during the Cretaceous Quiet Period (112 - 82 My b.p.), the task becomes harder, and other clues must be sought. The Pacific crust immediately east of the Tonga-Kermadec trench is one example of such crust (Larson and Chase, 1972). Another problem in reconstructions is that, with the commencement of subduction, a large volume of oceanic crust is consumed into the earth's interior, and with it all geological evidence of its past, such as ridges, transform faults and magnetic lineations. This is the case with the subduction of the Pacific plate around the Pacific margin (Larson and Chase, 1972). Further reference to the general tectonic history of the S.W. Pacific, and its relevance to this 


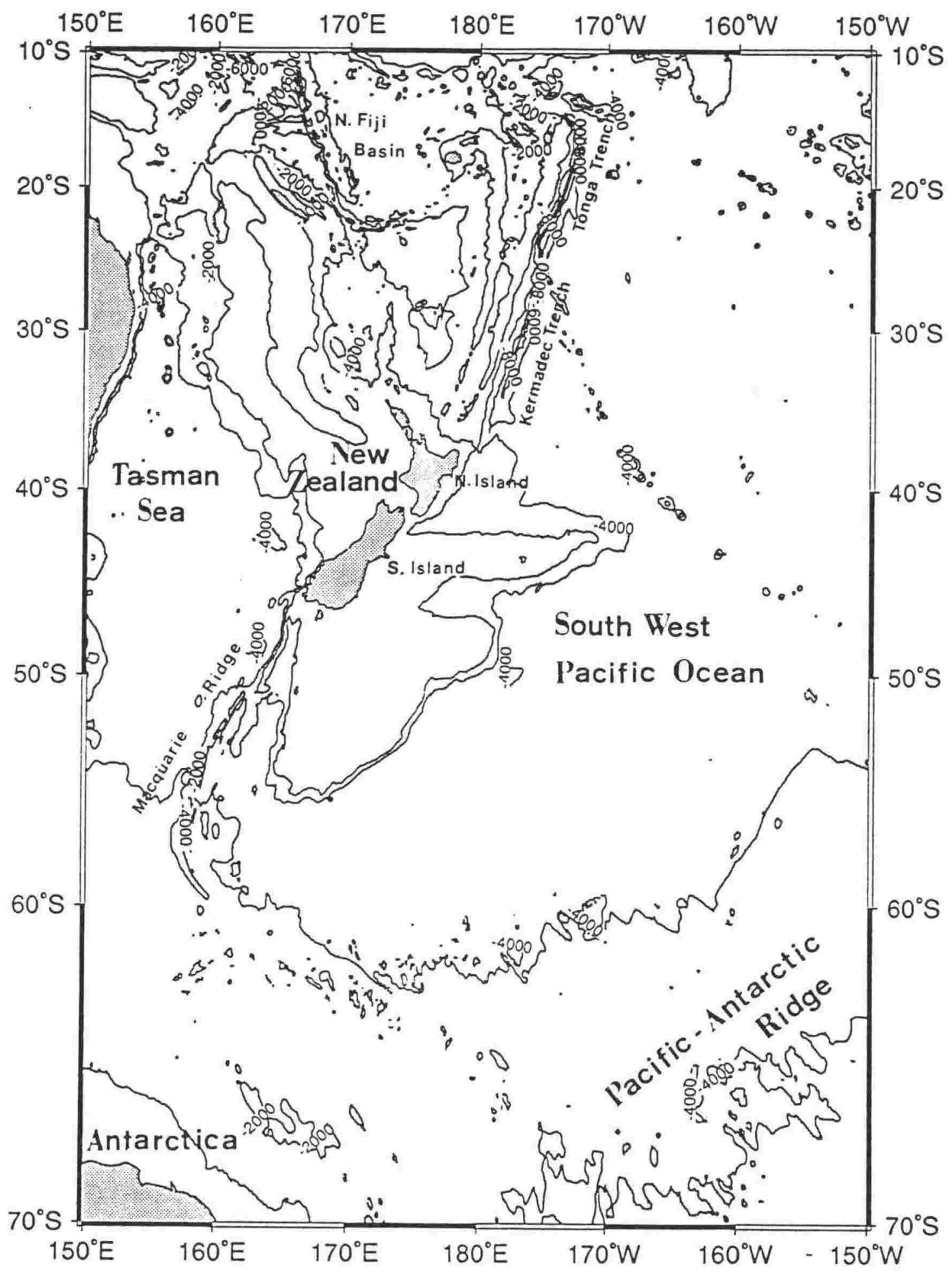

Fig. 1.1 Geographical setting of New Zealand in the south west Pacific ocean. 
Pacific, and its relevance to this thesis, is postponed to a later chapter.

Subduction zones themselves are regions of great geophysical and geological interest. The bent and dipping lithosphere and the overlying crust are subjected to a complex regime of stresses and to large changes in temperature and pressure, which manifest themselves in seismicity, volcanism, mountain building and back-arc spreading processes, and in chemical, physical and mechanical transformations to the lithosphere. The seismicity of subduction zones is a powerful tool for investigating the nature of such features and the processes taking place within them. Well located earthquake hypocentres help to delineate the broad geometry and morphology of subducted slabs, while the state of stress within the subducted lithosphere can, to a certain extent, be inferred from fault plane solutions of intraplate earthquakes. In recent years, tomographic inversion of earthquake travel times has been increasingly applied to image the velocity structure within regions of subduction throughout the world. One characteristic that has been shown to be common to all such regions is the presence of a high velocity anomaly with respect to the surrounding mantle, penetrating to hundreds of kilometres depth in correspondence with the cold descending lithosphere (e.g. Zhou, 1990; van der Hilst, 1991). The behaviour of materials under high pressures and temperatures can be duplicated in the laboratory to some extent, however the exact behaviour of lithospheric material in the most deformed sections of the descending slab are still not fully known.

\subsection{The Tectonics and Seismicity of New Zealand}

The convergent boundary between the Pacific and Indo-Australian plates extends to the north of New Zealand as the Tonga-Kermadec trench and subduction zone, and the Hikurangi trough off the eastern margin of the North Island represents the easternmost expression of the Pacific plate subduction in this region (Figure 1.2). Subduction beneath the North Island appears to come to an abrupt stop at approximately $42^{\circ} \mathrm{S}$, where the oceanic crust of the Pacific plate gives way to the continental-like crust of the Chatham Rise and Campbell Plateau (Adams, 1964; Cowan, 1992). In the southernmost South Island, subduction is resumed, however, here it is the Australian plate which descends below the Pacific plate underneath Fiordland and the Macquarie Ridge (Christoffel, 1971). The two subduction zones are linked by a major shear zone, the Alpine fault, which runs in a NE-SW direction through the South Island (Figure 1.2). The east-west motion of the Pacific plate with respect to the Australian plate results in oblique convergence at the rate of approximately $50 \mathrm{~mm} / \mathrm{yr}$ (Walcott, 1978). This motion is mostly accomodated by plate subduction, but due to the oblique character, the boundary zone in New Zealand is also characterized by a component of right-lateral transform motion. The relative direction of motion of the Pacific plate with respect to the Australian plate (Figure 1.2) is such that, on going further 


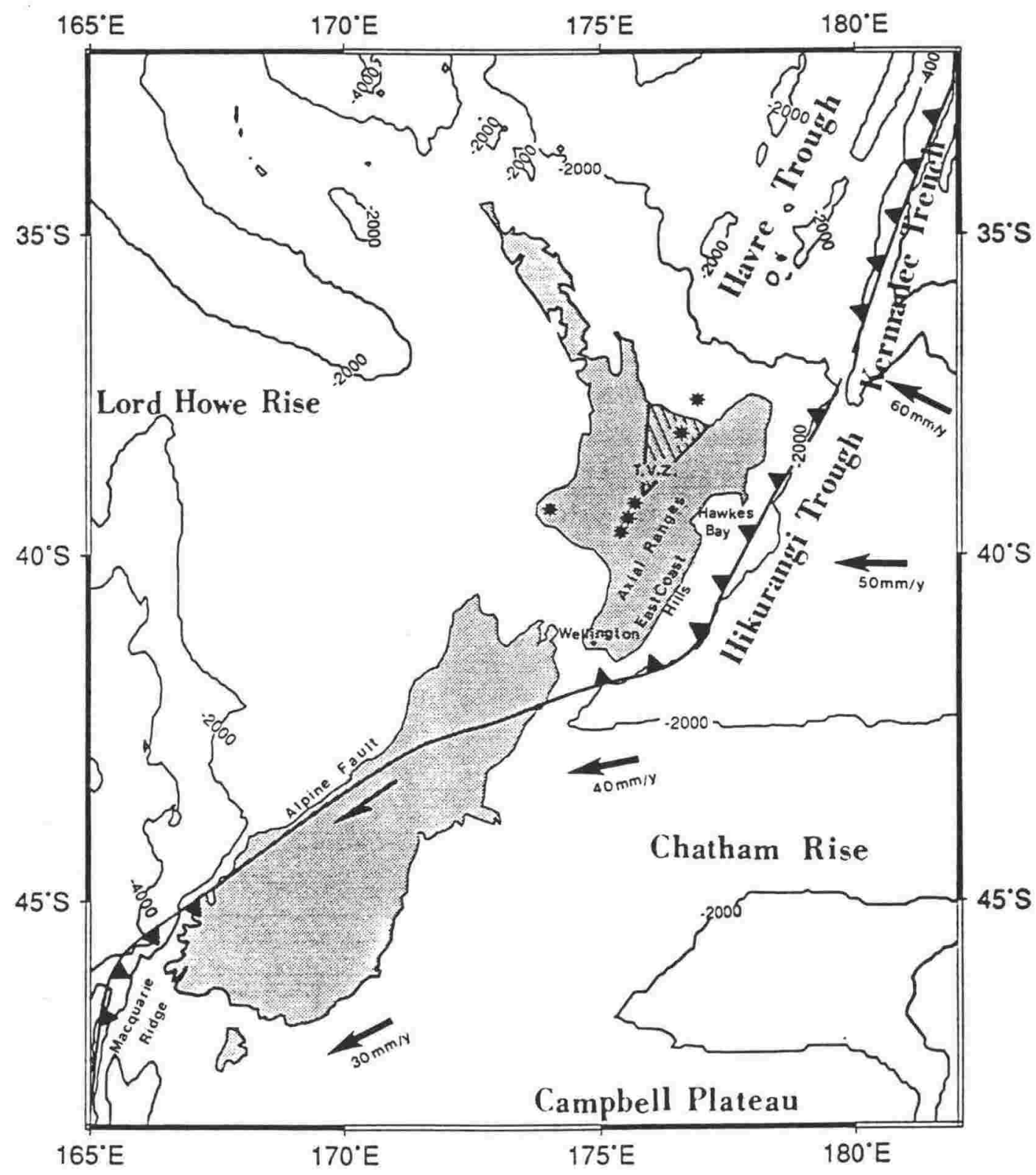

Fig. 1.2 Major tectonic and geological features of the Pacific/Indo-Australian plate boundary in New Zealand. TVZ = Taupo Volcanic Zone 
south, the convergence becomes increasingly oblique and parallel to the plate boundary, so that in the South Island, the motion is largely strike-slip (Cole and Lewis, 1981).

The plate boundary has been described by Walcott (1978) as forming an "axial tectonic belt" 70-100 km wide characterized by seismicity and pervasive deformation. Strain measurements throughout the country have confirmed the existence of an axis of compression along $\mathrm{N} 110^{\circ} \mathrm{E}$ within the axial tectonic belt (Walcott, 1978). This compression is taken up by crustal thickening and uplift, resulting in the Quaternary orogeny of the axial mountain ranges in the North Island and in the formation of the Southern Alps in the South Island.

The Havre trough west of the Tonga-Kermadec trench represents a young back-arc spreading centre associated with the subduction of the Pacific plate (Cole and Lewis, 1981). It is believed that this spreading ridge extends south to New Zealand, terminating at the Taupo rift zone, which is likewise a region of recent volcanism $(<1 \mathrm{My})$ and high heat flow. The Taupo rift zone also contains an active chain of andesitic volcanoes, running approximately parallel to the axial mountain ranges, and younger than $0.05 \mathrm{My}$ old. The sedimentary sequences and coastal hills along the North Island's east coast have been interpreted to represent the imbricate-controlled accretionary borderland (Cole and Lewis, 1981) formed by the interaction of the front edge of the Indo-Australian plate with the subducting Pacific plate. There is a general trend of progressive aging of the sedimentary sequence on going inland from the east coast.

Below the North Island, subduction gives rise to a band of seismicity within the plate, striking $\mathrm{N} 40^{\circ} \mathrm{E}$ and dipping to the north-west. There is a marked termination of this pattern of seismic activity below the northernmost South Island, in correspondence with the change of character of plate convergence, as discussed above. It is generally agreed that the upper limit of the dipping band of intense seismicity coincides with the upper surface of the slab and therefore the broad geometry of the descending plate is relatively well known. Its surface lies at shallow depths $(<20 \mathrm{~km})$ beneath the eastern coast of the North Island (Reyners, 1980) but below the central North Island, the dips at about $50^{\circ}$, with the upper surface reaching depths of more than $300 \mathrm{~km}$ off the western coast (Adams \& Ware, 1977). A map of deep seismicity is shown in Figure 1.3. The deeper limit of seismicity increases progressively from about $200 \mathrm{~km}$ below the northern South Island to about $350 \mathrm{~km}$ beneath the Bay of Plenty (Adams and Ware, 1977; Reyners,1989). By accurate relative relocation of hypocentres, Ansell and Smith (1975) demonstrate that the seismogenic thickness of the slab at depth may be as thin as $9 \mathrm{~km}$.

Shallow seismicity throughout New Zealand, associated mainly with deformation in the overlying plate, is more diffuse, and shows only a weak correlation with the major fault patterns. A map of the shallow seismicity is shown in Figure 1.4.

The seismicity of New Zealand is generally monitored by the New Zealand Seismological Observatory (NZSO), through the New Zealand National Seismic Network, for which the average station spacing is approximately $150 \mathrm{~km}$ (Figure 1.5). This network now consists almost completely of digital seismographs. Over the past 12 years or so, considerable improvement in 


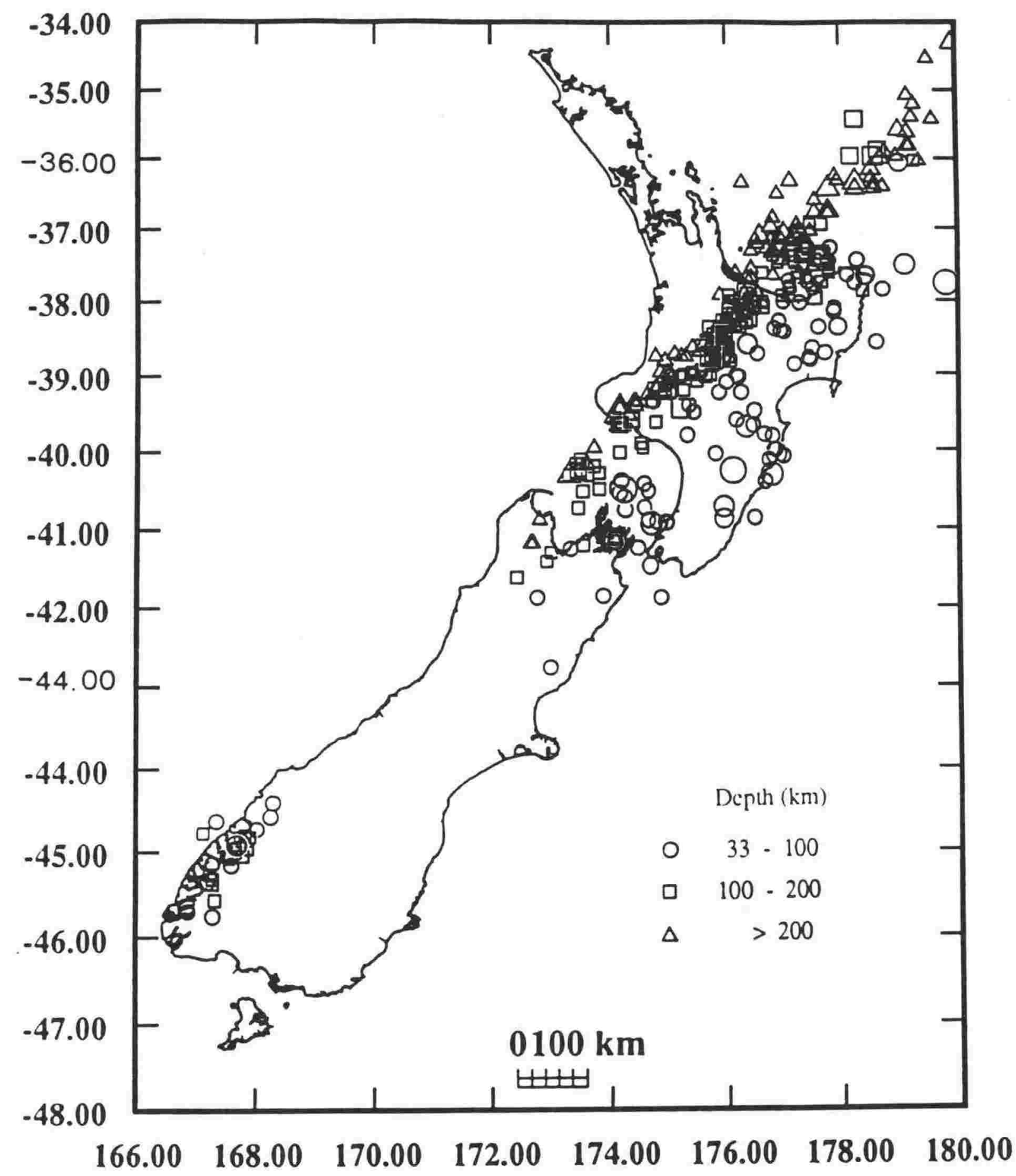

Fig. 1.3 Epicentres of earthquakes deeper than $33 \mathrm{~km}$ located by I.S.C. during 1986 
the knowledge of earthquake source parameters and crustal structure has been made through the use of temporary local networks of portable seismographs deployed for detailed microearthquake studies of particular areas. Also important have been the local, telemetered subnetworks of the national network, such as the ones in Wellington and Hawkes Bay. The Wellington network has been particularly fruitful in enabling a more detailed understanding of the subducted plate below the southern North Island (Robinson, 1986). The use of close-spaced networks is particularly important in enabling much more accurate depth estimates of crustal earthquakes, this being far less reliable with the large station spacing of the national network.

The microearthquake studies in the North Island have concentrated on the regions of Hawkes Bay (Reyners, 1979; Chong, 1982; Bannister, 1986), the Taupo Volcanic Zone (Reyners, 1979) and the Wairarapa region in the south east corner of the North Island (Kayal, 1983, 1986). There appears to be consistency between the results of these microearthquake studies as regards the position of the top surface of the subducted slab and its general geometry. The estimated depth of the plate interface varies between $10 \mathrm{~km}$ (Wairarapa) and $18 \mathrm{~km}$ (Hawkes Bay) below sea level along the east coast, increasing gently to about $25 \mathrm{~km}$ below the axial ranges, and then more rapidly to about $70 \mathrm{~km}$ below the Taupo Volcanic zone. A change in the dip of the plate from about $16^{\circ}$ to about $22^{\circ}$ is indicated below the axial ranges.

There is less agreement about the thickness of the subducted crust, which the above authors have associated with the main band of seismic activity below the plate interface, as well as inferred from the results of seismic velocity inversions. Kayal (1983) claims a thickness of 18-23 $\mathrm{km}$ for the "Benioff zone", increasing from south to north along the Wairarapa coast. Reyners (1979) and Bannister (1986) found thicknesses of $15 \mathrm{~km}$ and $12 \mathrm{~km}$ respectively in Hawkes Bay, while Chong (1982) arrives at $6 \mathrm{~km}$ of oceanic crust from his final velocity model obtained by inversion of earthquake travel times. A common feature of the pattern of seismic activity within the subducted lithosphere is the presence of a second band of far less intense activity below the main crustal band. This lower band is especially evident in the Hawkes Bay region, where it occurs between 40 and $70 \mathrm{~km}$ depth below sea level. Kayal (1983) reports this second band as persisting down the Wairarapa coast between 50 and $70 \mathrm{~km}$ depth.

In his microearthquake study of the Wellington region, using the Wellington network, Robinson (1986) also deduces the plate interface to lie at about $22 \mathrm{~km}$ depth below Wellington city, dipping at approximately $15^{\circ}$. The hypocentres lie within a dipping band of $15 \mathrm{~km}$ thickness. The hypocentre locations in this study are reliable enough to reveal a $7 \mathrm{~km}$ vertical offset of the plate interface along a NW - SE line through Cook Strait, which the author attributes to plate deformation as a result of the abrupt change in the subduction process that takes place further south. Robinson also observes the double-banded seismic zone within the Pacific plate, the two zones being separated by about $20 \mathrm{~km}$, and the lower zone again being far less intense than the upper one.

The determination of focal mechanisms of intraplate earthquakes has consistently revealed 


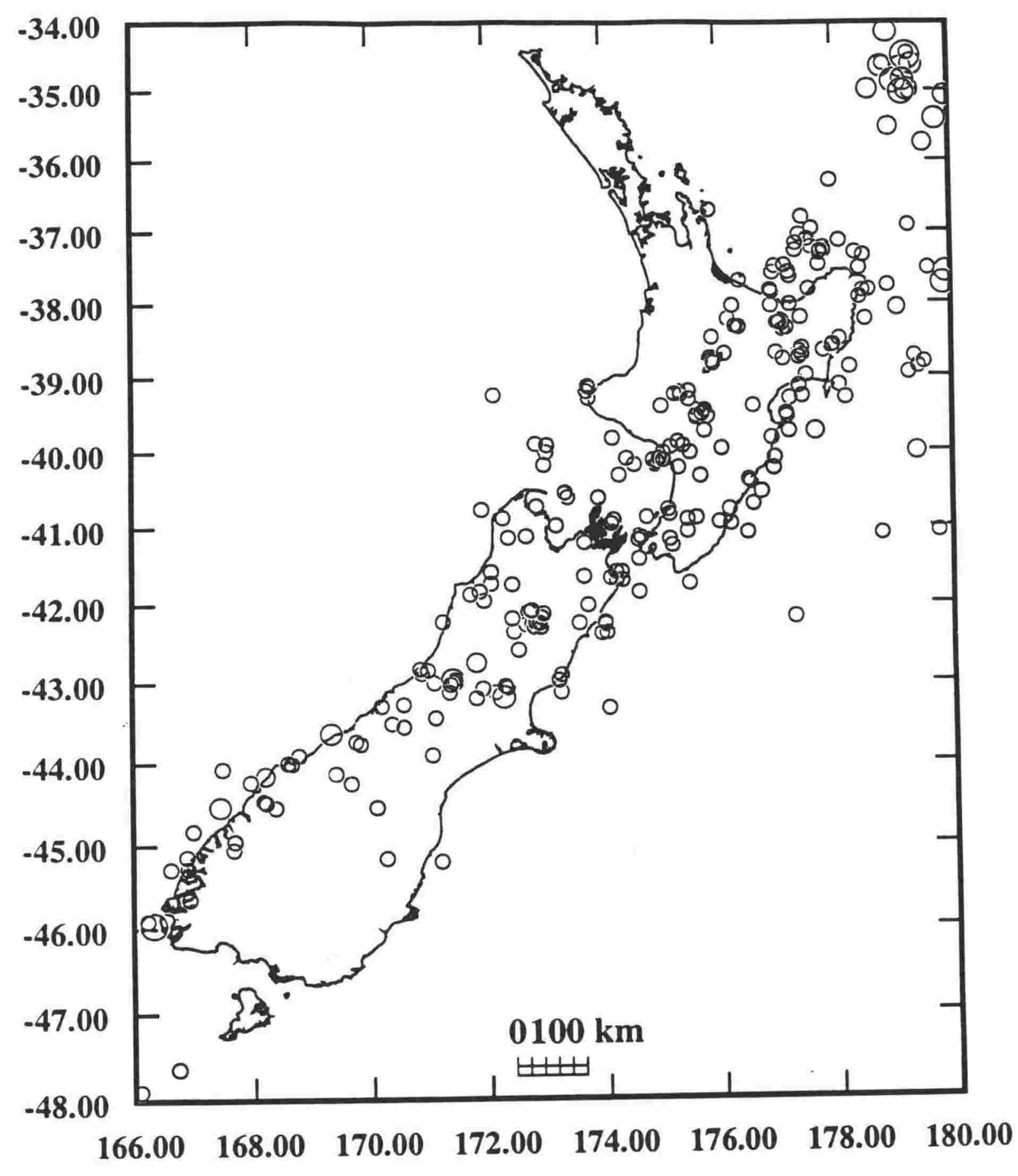

Fig. 1.4 Epicentres of earthquakes shallower than $33 \mathrm{~km}$ located by I.S.C. during 1986 
that the top $15 \mathrm{~km}$, or so, of the shallow subducted slab below the North Island is affected mainly by tensional effects due to slab pull and curvature of the lithosphere. This is evident in the predominance of normal faulting with the T-axis parallel to the dip of the slab (Chong, 1982; Kayal, 1983; Robinson, 1986; Bannister, 1988). Deeper down, there is some evidence that the stress pattern within the subducted plate may reverse, with thrust faulting becoming the dominant mechanism, and the P-axis aligned downdip. There are very few earthquakes which have been analysed at this depth for focal mechanism. Bannister (1986) found one event, in Hawkes Bay, at $51 \mathrm{~km}$ depth that displayed this mechanism, while Chong (1982) reports two such events in the Dannevirke region at depths between 50 and $64 \mathrm{~km}$ depth. Bannister $(1986,1988)$ interprets these mechanisms in terms of a flexure model of the subducting oceanic lithosphere of approximate elastic thickness $50 \mathrm{~km}$. Following the analysis of bending plates by Chapple and Forsyth (1979), the observed mechanism patterns would be consistent with a "neutral surface" at $45-50 \mathrm{~km}$ below sea level in the $\mathrm{S}$. Hawkes Bay region, at which the bending stress pattern changes over from extensional downdip in the upper (convex) part of the plate, to compressional downdip in the concave curved lower part. This is somewhat in disagreement with findings by Reyners (1984) who gathers information about focal mechanisms of 5 previous large earthquakes occurring at or below $40 \mathrm{~km}$ depth along the east coast of the North Island. For these earthquakes, the faulting mechanism is predominantly normal faulting. There is no indication, however, as to the extent by which the depths of these events may be in error. In the same publication, Reyners describes results of an aftershock study of a magnitude 5.6 subcrustal event, occurring in S. Hawkes Bay. For the aftershocks, located between 40 and $60 \mathrm{~km}$ depth with the use of a portable microearthquake network, a composite first motion plot does not yield a single solution consistent with any one mechanism, suggesting that normal faulting is probably not the only kind of deformation taking place. In the Wellington region, the events in the deeper seismic band located by Robinson show no indication of thrust faulting. A composite first motion plot of these events reveals a tension axis downdip and a compression axis along strike, resulting in right lateral shear.

In the overlying Australian plate, the predominant focal mechanism is right-lateral strike slip in the Wellington region (Robinson, 1986), with an east-west directed compression axis, parallel to the direction of convergence. Further towards the east coast, however, the deformation appears to be more dominated by thrust faulting, again with an east-west oriented axis of compression. Figure 1.6, taken from Reyners (1979) is a schematic illustration of the types of deformation that may be associated with the bending Pacific lithosphere. 


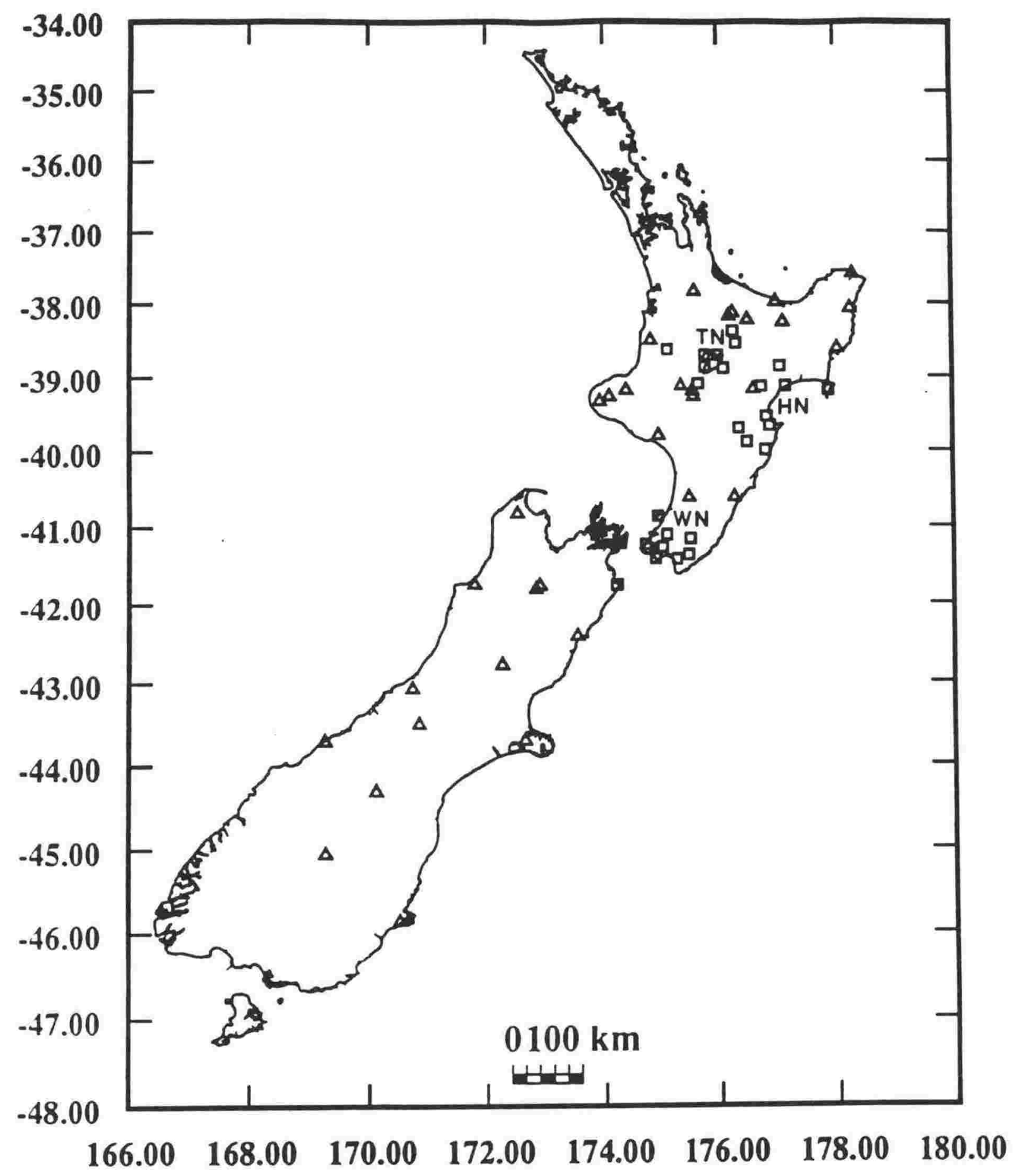

Fig. 1.5 Station distribution for the National Digital Seismic Network of New Zealand. Stations shown as squares form part of regional subnetworks. TN = Taupo Network; HN = Hawkes bay Network; $W N=$ Wellington Network 


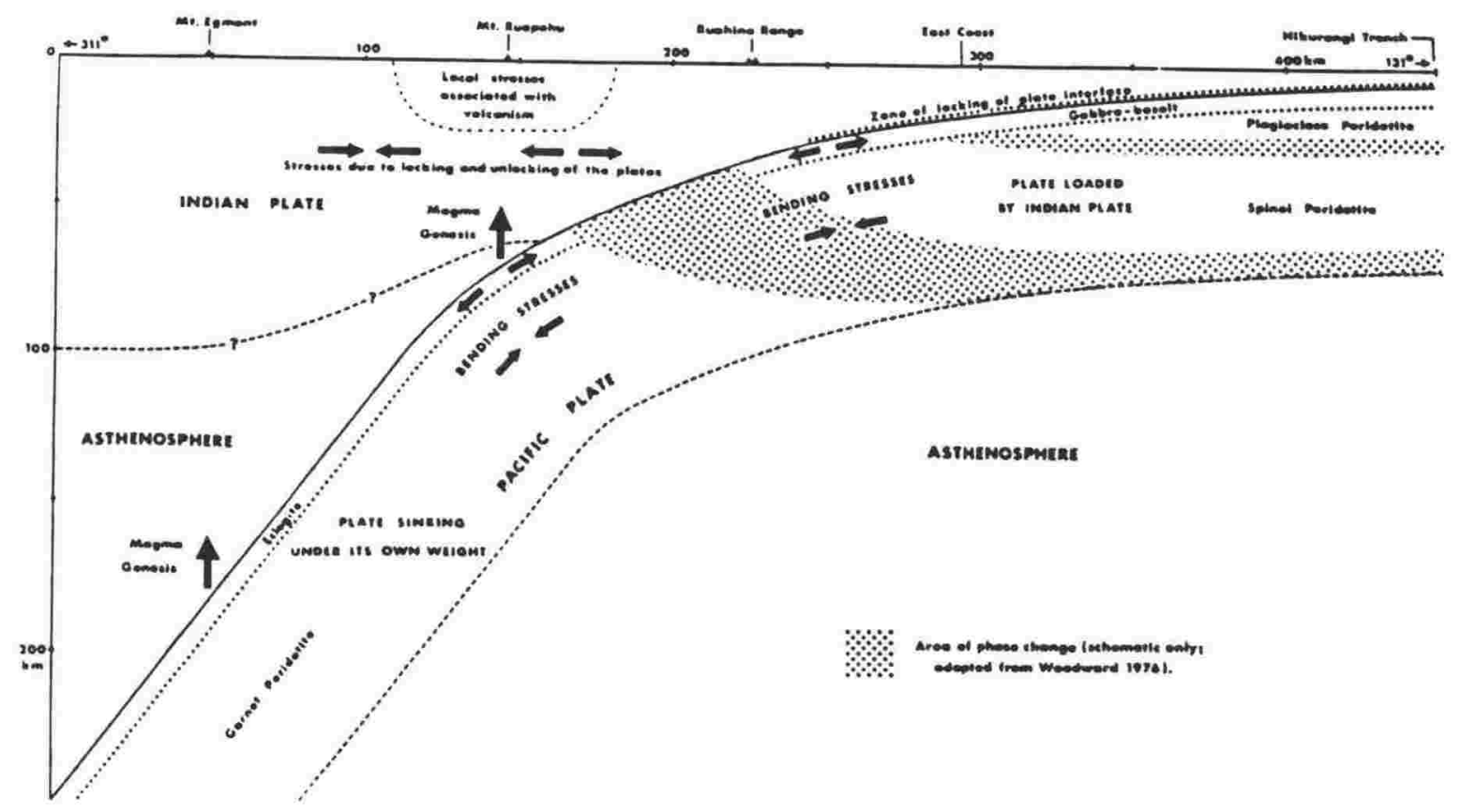

Fig. 1.6 Postulated deformation patterns in the subducted plate, from Reyners (1979)

\subsection{Velocity Models for New Zealand}

The setting up of an adequate velocity model suitable for the whole of New Zealand is unrealistic, in view of the complexity of structures and the large extent of lateral heterogeneity. Nonetheless, un:il recently the location of earthquakes all over New Zealand by the National Seismic Network made use of one, laterally homogeneous, horizontally-layered model (Table 1.1). This resulted in inconsistencies in hypocentre locations, especially in depth. One major reason is the presence of the high velocity anomalies associated with the subducting Pacific lithosphere (Ansell, 1978). Behind the Taupo Volcanic Zone, on the other hand, velocities are lower than average, and the material is highly attenuating (Mooney, 1970), being the probable continuation of the low-velocity anomaly beneath the Lau-Havre trough and South Fiji Basin (Mitronovas and Isacks, 1971). Adams and Ware (1977) reduced the inconsistencies in hypocentre locations by using a velocity model which took into account the higher average velocities associated with the descending lithosphere. In their model the velocities of both $\mathrm{P}$ and $S$ waves in the Benioff zone are increased by $11 \%$ over the standard Jeffreys-Bullen model. 
Table1.1 The New Zealand standard velocity model

\begin{tabular}{|ccc|}
\hline $\begin{array}{c}\text { Depht to top } \\
\text { of layer } \\
(\mathrm{km})\end{array}$ & $\begin{array}{c}\text { P-wave } \\
\text { velocity } \\
(\mathrm{km} / \mathrm{s})\end{array}$ & $\begin{array}{c}\text { S-wave } \\
\text { velocity } \\
(\mathrm{km} / \mathrm{s})\end{array}$ \\
\hline 0.0 & 5.5 & 3.3 \\
12.0 & 6.5 & 3.7 \\
33.0 & 8.1 & 4.6 \\
\hline
\end{tabular}

With the deployment of microearthquake networks, considerably more detailed knowledge has been gained of the seismic velocity variation in the crust and upper mantle below several regions. Several such studies have been carried out in the lower half of the North Island. In his Wellington study, using a joint velocity-hypocentre inversion for earthquakes located over a 6-year period by the Wellington network, Robinson (1986) arrived at a reliable 3-dimensional working model for the velocity structure below the Wellington region (Figure 1.7a). His model is now routinely used in the location of earthquakes in the Wellington region. The model also shows in itself a variation of character from east to west, notably a decrease in average crustal $\mathrm{P}$ velocity on going from the west to the east bounds of the model.

Simultaneous least squares inversions of arrival times for velocity structure were also carried out by Chong (1982) and Bannister (1986) in their microearthquake surveys, and their final results are shown in Figures $1.7 \mathrm{~b}$ and 1.7c. From the models of Figure 1.7, it can be seen that, whereas results about the geometry of the subducted plate are quite consistent among the various studies, the velocity models deduced show a considerable variation, both in the average velocities as well as in the distribution. An interesting feature of Bannister's model is the thin layer of low velocity material forming the upper surface of the subducted plate, which he interprets as oceanic sediment being drawn down together with the descending lithosphere. This feature has also been inferred by Xun (1992) for the subducted crust off Cape Palliser at the southeast point of the North Island. Such low velocity layers have also been modelled in subduction zones below Japan (Hori, 1990) and the west coast of North America (Mundal et al,1990).

The New Zealand standard model of Table 1.1 is still used to an extent for hypocentre locations throughout New Zealand, but in "special areas" where the velocity structure has been inferred in more detail, the model for that area is used in the inversion.

A feature of the velocity distribution which appears to result from most attempts to calculate oceanic upper mantle velocities is the presence of unusually high P-wave velocities at some depth within the subducted lithosphere below the North Island. Velocities of $8.68 \mathrm{~km} / \mathrm{s}$ and $8.61 \mathrm{~km} / \mathrm{s}$ 


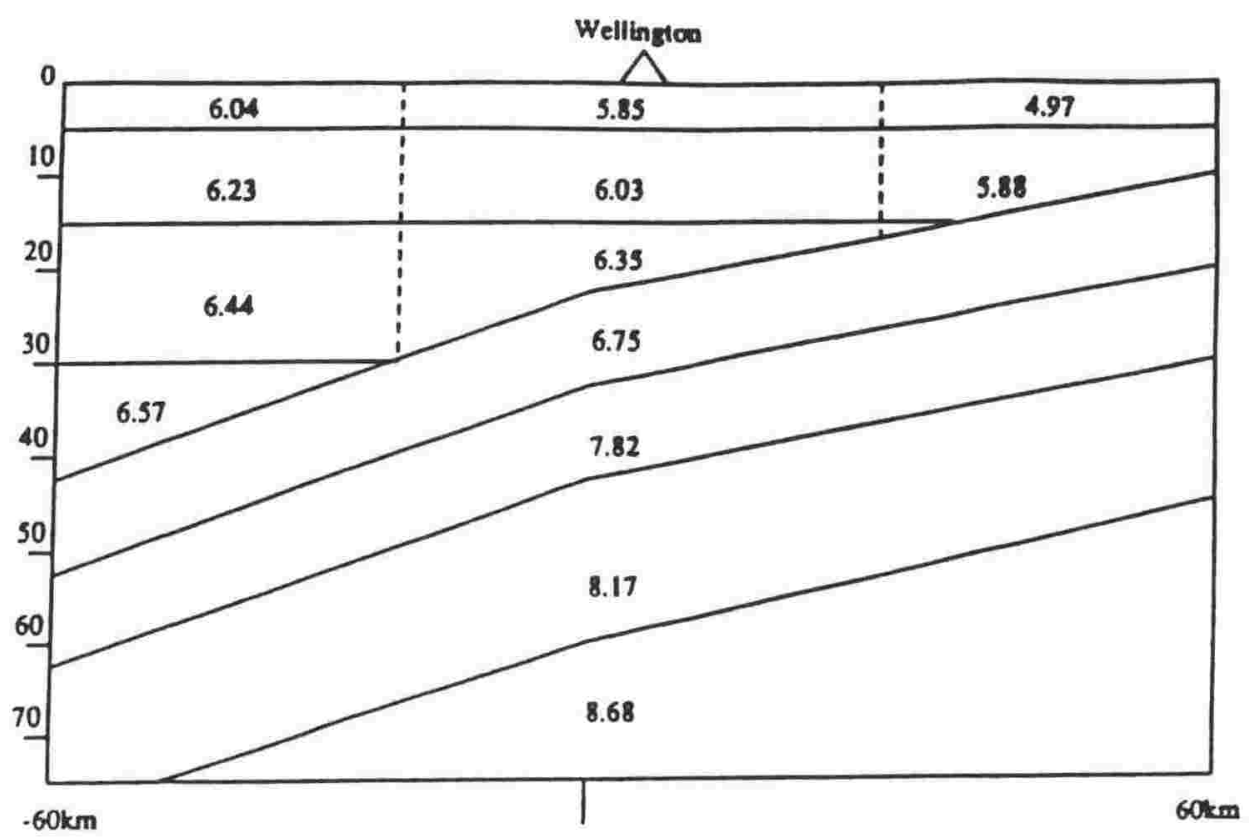

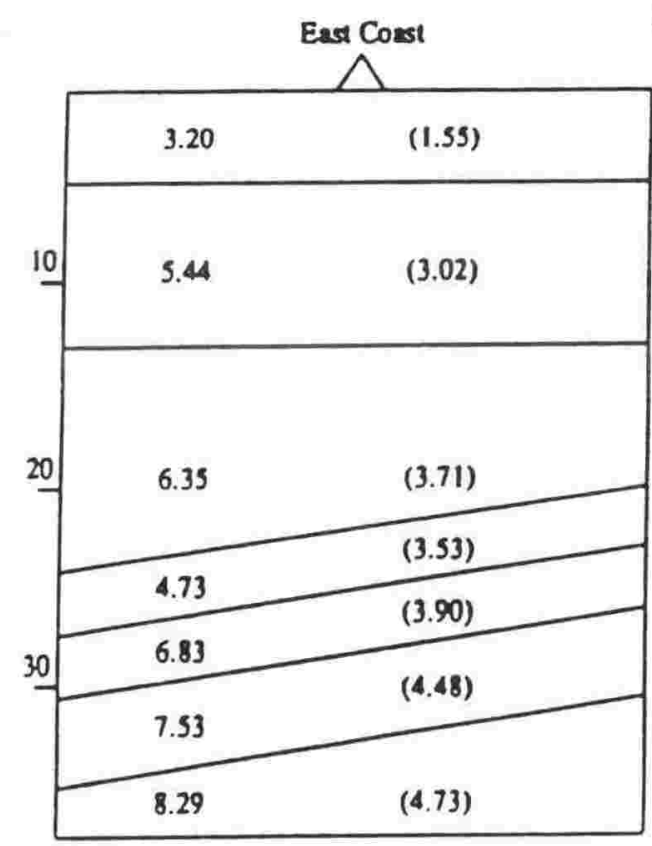

(b) (a)

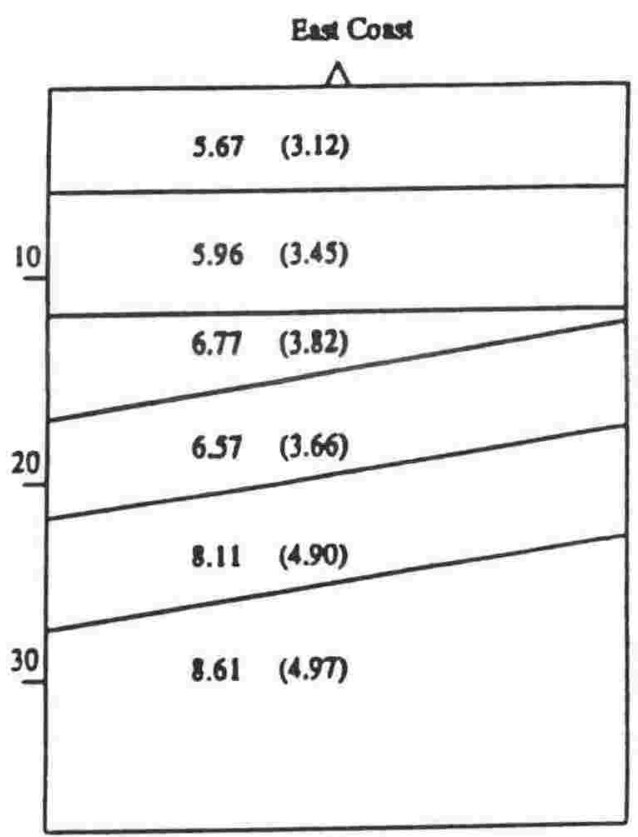

(c)

Fig. 1.7 Velocity models in the lower half of the North Island from inversion of earthquake travel times in recent microearthquake surveys. Values in parentheses denote $S$-wave velocities.

(a) Wellington Region, Robinson (1986)

(b) Northern Hawkes Bay, Bannister (1986)

(c) Southern Hawkes Bay, Chong (1982) 
result from the velocity inversions of Robinson (1986) and Chong (1982) (Figures 1.7a and 1.7c), while in his inversions, Bannister also arrives at one possible model which has an upper mantle Pwave velocity of $8.76 \mathrm{~km} / \mathrm{s}$. Most attempts to measure the upper mantle velocities, however, have made use of arrival times of critically refracted $P_{n}$ waves at a set of stations, from shallow earthquakes more than $100 \mathrm{~km}$ away. The most comprehensive work on upper mantle velocities remains that of Haines $(1976,1979)$, who regionalised $P_{n}$ and $S_{n}$ velocities all over New Zealand. using time-term analysis. His results are shown in Figure 1.8. The high average $P_{n}$ velocity of $8.5 \mathrm{~km} / \mathrm{s}$ for the south eastern North Island is significant. Moreover, some individual path velocities from which the average is calculated are as high as $8.7 \mathrm{~km} / \mathrm{s}$, after correction for station terms have been carefully made. Such velocities are in line with other observation of high $P_{n}$ velocities in this region. Dibble (1957) reports one of the earliest such observations - $8.7 \mathrm{~km} / \mathrm{s}$ from earthquakes between New Zealand and the Kermadec trench recorded along a line through Wellington, at distances greater than $4^{\circ}$. Kayal and Smith (1989), from travel-time differences,

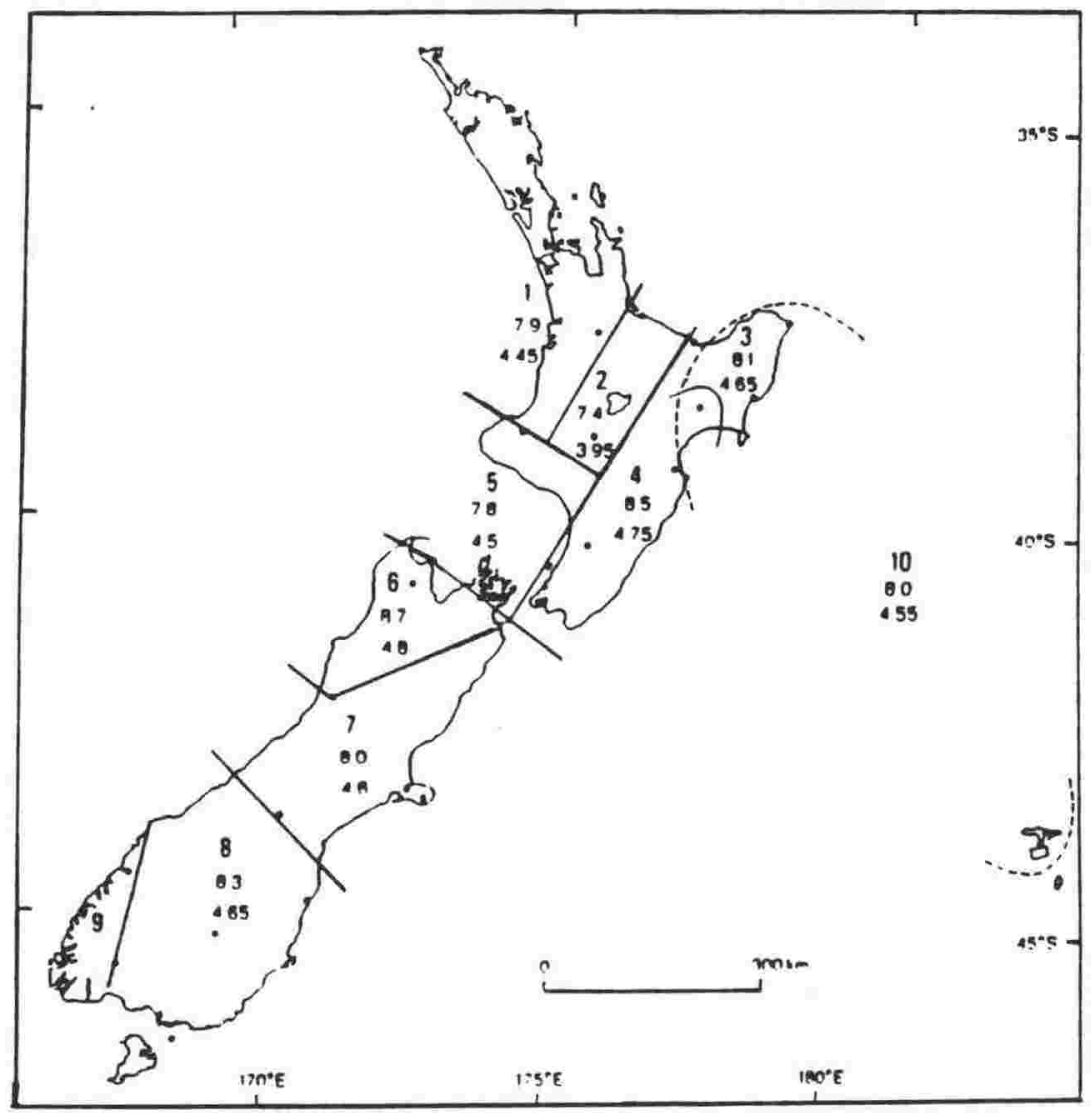

Fig. 1.8 Upper mantle $P$ and $S$ velocities, from Haines (1979) 
measured $\mathrm{P}_{\mathrm{n}}$ apparent velocities of up to $8.9 \mathrm{~km} / \mathrm{s}$ downdip along the subducted slab in the Wairarapa region. Bannister (1986) and Chong (1982), in their microearthquake surveys also make use of $P_{n}$ waves from more distant earthquakes recorded across their seismic arrays, and observe $P_{n}$ velocities of around $8.6 \mathrm{~km} / \mathrm{s}$ in this way.

Fast P-velocities have been measured along strike of the subducted Pacific plate further north, below the Tonga-Kermadec trench (Aggarwal et al,1972;Ansell and Gubbins, 1986; Gubbins and Snieder, 1991) and are often attributed to the transformation of basalt, making up the oceanic crust, to the high-density eclogite. However, whether there is any relation between this feature and the locally higher velocities below the lower half of the North Island is to be determined. In particular, it is desirable to seek a rheological/mineralogical explanation for these higher seismic velocities, and how, if at all, they are related to processes occurring within the subduction of the Pacific lithosphere.

A major step has recently been taken towards a better understanding of the nature of the descending Pacific lithosphere below New Zealand. A large-scale seismic refraction experiment, referred to as the Hikurangi Margin experiment, was undertaken jointly by the I.N.G.S. (Institute of Geological and Nuclear Sciences, formerly D.S.I.R. Geology and Geophysics), the Geophysics Institute of Victoria University of Wellington, the Geological Survey of Canada and the University of Leeds (Chadwick and Reyners, 1992). The refraction line consisted of some 100 stations, spread along a $270 \mathrm{~km}$ stretch down the east coast of the North Island, parallel to the strike of subduction. Processing and analysis of the data are now under way, and are expected to yield important results.

\subsection{Outline of this Study}

In this study, several aspects of the subducted slab structure and velocity characteristics are explored, using a variety of techniques and data. In Chapters 2 and 3, the data used are mainly those from an earthquake and aftershock sequence in southern Hawkes Bay, recorded on a temporary, close-spaced network of portable seismographs in Wellington. These data are used to confirm the existence of unusually high $\mathrm{P}$ velocities in the mantle of the Pacific lithosphere, and also provides a clear image of off-azimuth first arrivals due to lateral refraction off the dipping slab. The combination of possible slab geometries and velocity structure are explored (Chapter 3 ) through modelling of the observed wavefront speed and azimuth at Wellington.

Whereas the results of Chapter 3 rely on the arrival times of the first phases on the seismograms, in Chapters 4 and 5, attention is turned to the whole waveforms, in particular the Pwavetrain. The overall character of the waveforms is distinctive and consistent for the whole data set. This has enabled the use of synthetic seismogram modelling, using a reflectivity method. 
Although seismograms of shallow earthquakes are notoriously difficult to model, the approach here has been to explain the gross features, such as the main energy arrival groups and frequency content. The first phases on the seismograms are sufficiently stable features as to enable somewhat more detailed waveform modelling and hence more detailed information about the velocity structure in the lowermost crust and upper mantle of the oceanic lithosphere, where these features originate.

In Chapter 6 we look farther afield, using a completely different data set, from the TongaKermadec trench, to elicit information about upper mantle velocities in the stable Pacific ocean lithosphere before it begins to subduct below the Tonga-Kermadec arc and New Zealand. The emphasis is on searching for anisotropic P-wave behaviour.

Chapter 7 contains a discussion of the results and of the possible relationship between the wave velocities in the subducted and the pre-subduction Pacific lithosphere. 


\section{Chapter 2}

\section{ARRAY MEASUREMENT OF APPARENT VELOCITIES ALONG STRIKE OF THE SUBDUCTED SLAB}

\subsection{Introduction}

In this chapter, some data sets are presented which provide evidence of the presence of very high seismic velocities at some level within the subducted Pacific lithosphere below the North Island. The main data set, referred to as the Weber data set, was a fortuitious occurrence because a large distant $(175 \mathrm{~km})$ earthquake and its aftershocks occurred during a period when a closelyspaced, 3-component digital seismograph network happened to be deployed in the Wellington region. This network will be referred to as the L-network. This data, apart from being numerous, yielded high quality seismograms on the small network, which enabled good estimates to be made of wavefront velocity, and later, modelling of the waveforms, for some conclusions to be drawn about the velocity structure (Chapter 5). Use was also made of other data collected on the L-network during the whole period of its operation.

Most of the data that has been used are associated with wave paths that are sub-parallel to the strike of the subducted slab.

\subsection{The L-Network}

From 2 March, 1990, a temporary L-shaped network of seven portable, digital seismographs had been set up in the Wellington region for a separate, local study (Gledhill, 1991). Figure 2.1 shows the location of the stations making up the L-network, as well as some of the stations of the Wellington network operated by the NZSO, while Table 2.1 lists their site properties. The L-network stations had a mean spacing of $1.1 \mathrm{~km}$, and were all sited on outcrops of Mesozoic greywacke. The stations consisted of $1 \mathrm{~Hz}, 3$-component Mark Products L4C seismometers, recording on portable versions of EARSS (Equipment for the Automatic Recording of Seismic Signals) instruments (Gledhill, Randall and Chadwick, 1989). The EARSS instruments have high dynamic range (>120dB), being equipped with gain-ranging amplifiers. Timing is kept by an internal clock, and corrected hourly against time signals from local radio stations. In this survey, the sampling frequency was set to $100 \mathrm{~Hz}$. EARSS instruments are now used in almost all the permanent seismograph stations of the National Network of New Zealand. 
Figure 2.2 shows the displacement response of a typical seismometer/EARSS instrument combination (Chadwick, 1991)

Table2.1 Location and component data for the L-network and for the 3 stations of the Wellington network used in this study (taken from Gledhill, 1991)

\begin{tabular}{|cccccc|}
\hline $\begin{array}{c}\text { Station } \\
\text { Code }\end{array}$ & $\begin{array}{c}\text { Latitude } \\
\mathrm{S}\end{array}$ & $\begin{array}{c}\text { Longitude } \\
\mathrm{E}\end{array}$ & $\begin{array}{c}\text { Elevation } \\
(\mathrm{m})\end{array}$ & Components & Lithology \\
\hline PDD & 41.2832 & 174.7263 & 190 & ZNE & Greywacke \\
SAL & 41.2977 & 174.7190 & 220 & ZNE & Greywacke \\
RKK & 41.3049 & 174.7110 & 100 & ZNE & Greywacke \\
KSE & 41.3107 & 174.7043 & 90 & ZNE & Greywacke \\
SEF & 41.3058 & 174.6874 & 75 & ZNE & Greywacke \\
EGB & 41.3035 & 174.7190 & 350 & ZNE & Greywacke \\
NDE & 41.2996 & 174.6496 & 375 & ZNE & Greywacke \\
& & & & & Greywacke \\
WEL & 41.2861 & 174.7683 & 122 & ZNE & Greywacke \\
CAW & 41.1088 & 175.0678 & 330 & Z & Greywacke \\
MRW & 41.2325 & 174.7050 & 235 & ZNE & Gy \\
\hline
\end{tabular}

Data recorded by the EARSS instruments is stored in the field on 20Mbyte magnetic cartridge tapes, which are later read into a PC-based event sorting and filing system (Gledhill,1991). From there, the data may be converted into an ah format for use in the seismic processing and analysis packages available on the SUN workstations of the Institute of Geophysics.

The Wellington seismograph network, established in 1976 by the Geophysics Division of the D.S.I.R. (now I.N.G.S.) consists of 11 seismograph stations, mostly 1-component, spread over the Wellington/Wairarapa/Marlborough regions, which relay data to a central site in Wellington via radio telemetry or telephone line (Robinson, 1975). The seismic signals are recorded on a SNARE network recording system (Gledhill and Randall, 1986), similar in principle to EARSS. The Wellington network records at $50 \mathrm{~Hz}$ sampling, as do the EARSS stations of the National Network. 


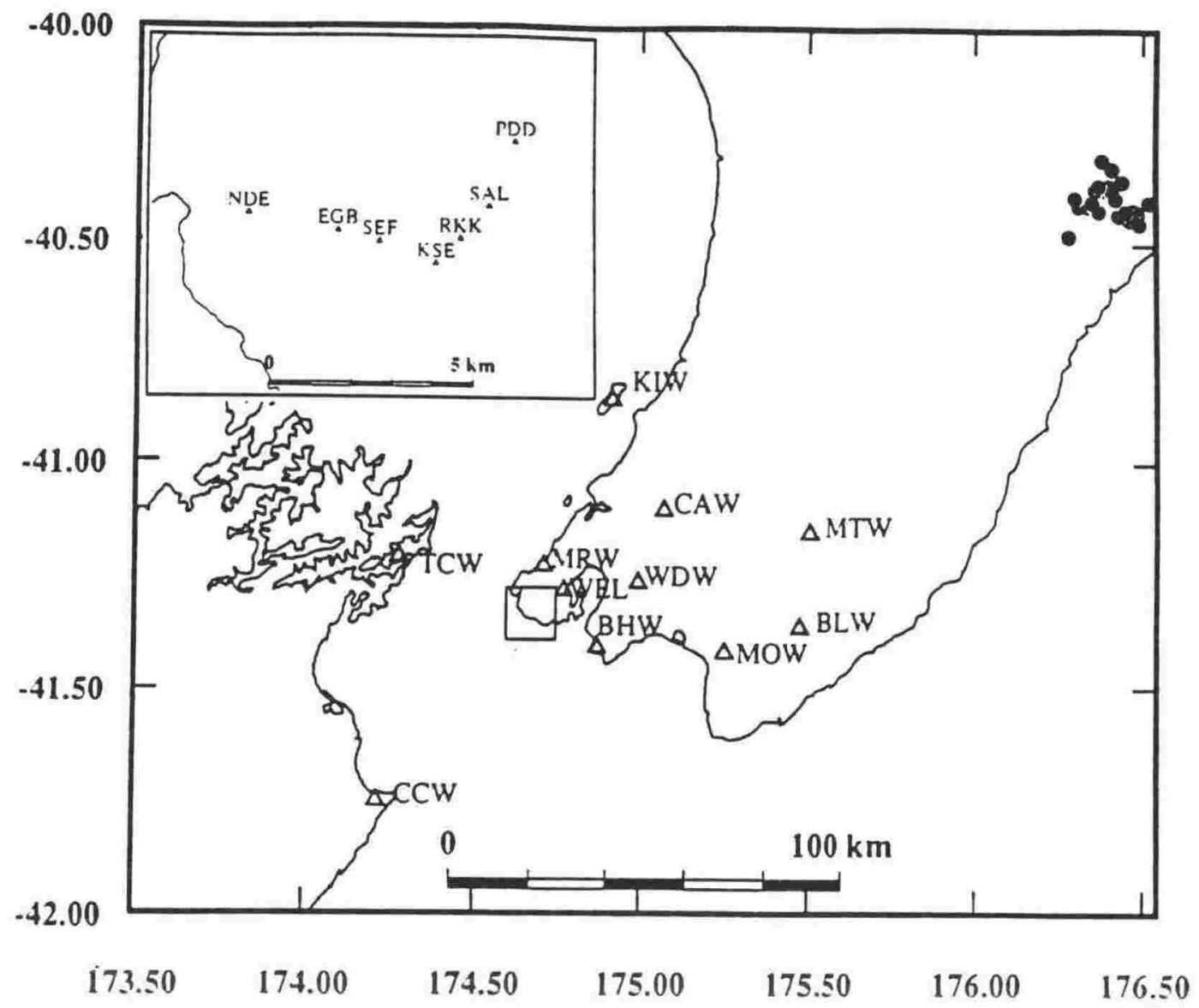

Fig. 2.1 Location of the L-network and some of the Wellington network stations. Inset shows the L-network configuration. Also shown are the locations of aftershocks of the 13 May 1990 Weber earthquake

\subsection{The Data Sets used}

\subsubsection{The Weber Earthquake Sequence}

The area of Southern Hawkes Bay was particularly active during 1990, being affected by two earthquakes of magnitude greater than 6 in a 3-month span. The aftershock sequences of these two events lasted for several months. Further activity occurred later in the year, including a magnitude 5.7 event on 15 August 1990 and a magnitude 4.5 event on 24 September 1990, all in the same region.

The first large earthquake $(M=6.1)$ occurred on 19 February 1990 , and the second $(M=6.3)$ on the 13 May 1990, both close to the small town of Weber in the S. Hawkes Bay region. The epicentres of these two earthquakes as given by the National Network locations were $44.40^{\circ} \mathrm{S}$, 176. $33^{\circ} \mathrm{E}$ and $40.34^{\circ} \mathrm{S}, 176.27^{\circ} \mathrm{E}$ respectively. They were also reported to have occurred at different depths, the first at $35 \mathrm{~km}$, within the subducted Pacific plate, and the second at $19 \mathrm{~km}$ depth, within the overlying Australian plate. For the second, shallower earthquake, the hypocentre solution is expected to be less reliable, since the nearest station used in the location, 


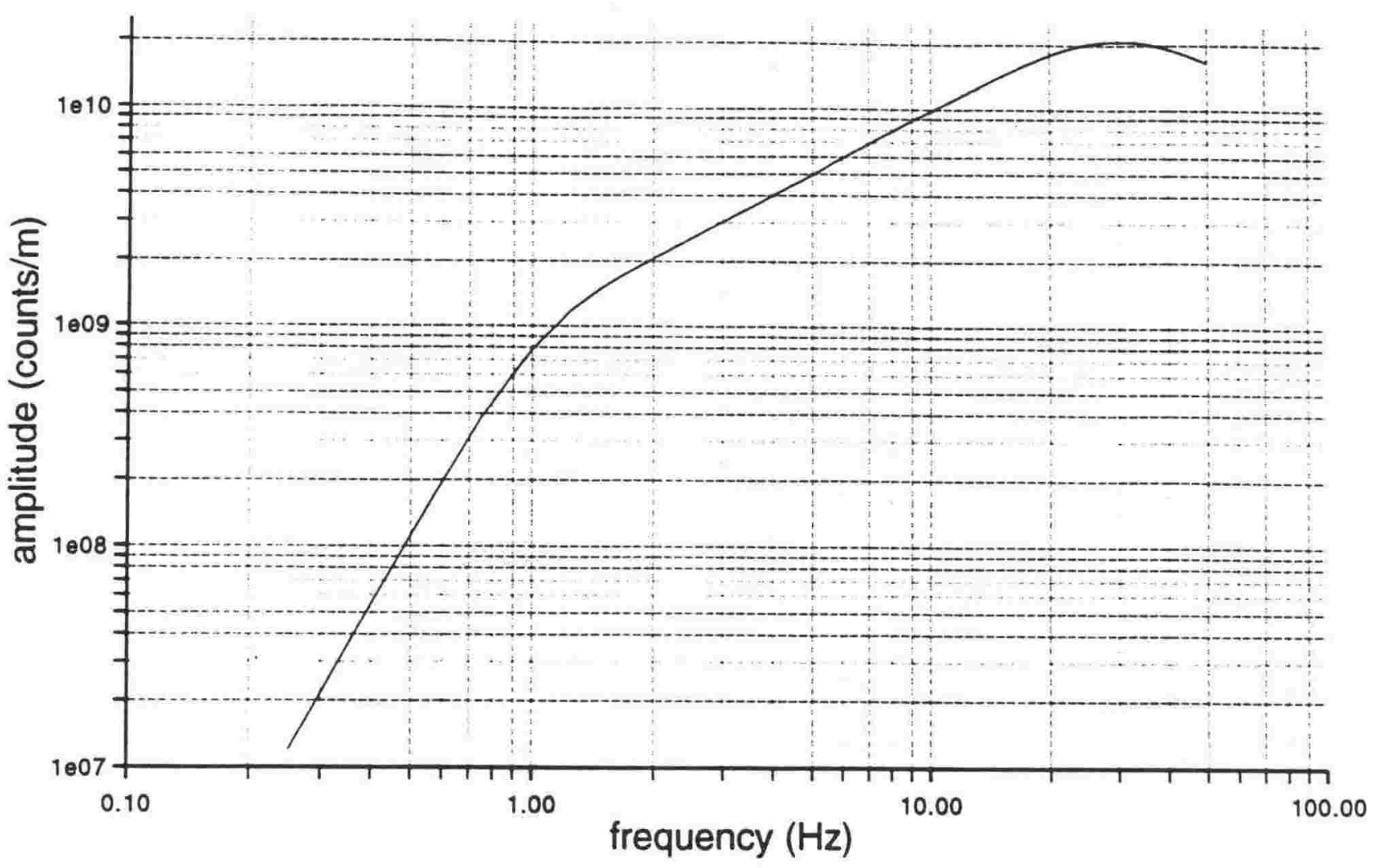

Fig.2.2 Displacement response of a typical EARSS seismograph

PGZ, was about $40 \mathrm{~km}$ away.

At the time of the May earthquake, the activity from the February event was still ongoing, so that after the May event, two groups of aftershocks were occurring at the same time, at two different levels within the crust. During its period of operation, the L-network recorded the aftershock activity of the February event, the mainshock occurring on the $13 \mathrm{May}$, and numerous aftershocks of this second event occurring on the same and few ensuing days. After that, most of the instruments had to be pulled out for use in the aftershock survey in the epicentral region. In all, more than 110 aftershocks following the 13 May event were large enough to trigger the $\mathrm{L}$ network before it was completely dismantled. The minimum magnitude of such events was 2.6 , but the large majority of events had magnitude larger than 3.0. During the period of operation of the network prior to the May event (66 days), only 16 events of the 19 February aftershock sequence triggered the network. There is evidence (Robinson, 1994) that the deeper level of activity increased after the May event, however, during the first and second days of the May sequence (which yielded most of the data used in this study), events in the shallow group well 
outnumbered those in the deeper group.

The L-network began to be dismantled at about 10:00 UT of the 13 May 1990. Thus recordings of the 13 May aftershocks on the full network are available for about 5 hours after the mainshock, during which time there were approximately 40 events that triggered the network. During the following 48 hours, about 65 further aftershocks were recorded on the partial network consisting of PDD, SAL, KSE, NDE and a further 11 events were recorded on the remaining tripartite network PDD, KSE, NDE. Unfortunately, station EGB was inoperative at the time of the mainshock and ensuing days, so no data are available from it for this data set.

In this section attention will be focussed on the set of earthquakes immediately following the 13 May event, and the data set will be referred to as the Weber data set. The epicentres of these earthquakes were, on average, $175 \mathrm{~km}$ away from Wellington, and the wave travel path was close to the strike of the subducted slab in the North Island. The direct line between the L-network and the epicentres made an angle of approximately $11^{\circ}$ downdip with the strike of the subducted slab.

The deployment of a microearthquake network consisting of 12 closely-spaced portable seismographs in the epicentral region shortly after the 13 May mainshock occurrence, ensured that accurate hypocentre locations could be made of the aftershocks. Thus a much more reliable image could be made of the extent and nature of the source region than would have been possible with the National Network alone. Of course it is unfortunate that for the mainshock and earlier aftershocks (whicn were recorded on the L-network and used for analysis in this study) the locations were far less reliable, being done with the use of the National network stations alone. This is especially a problem for the shallower events. Thus for the data set used, it is not possible to identify the aftershocks occurring in the deeper group (and associated with the February event) on the basis of reported hypocentre depths alone. More accurate locations, using the portable network stations, are available from about 16 hours after the mainshock, at approximately 00:00 UT of the 14 May 1990. Before this time, more than 350 aftershocks had been located routinely by the National Network.

Although the locations of the mainshock and earlier aftershocks are probably in error, it is possible to get an estimate of the extent of their mislocation. Figure 2.3 shows two separate sets of aftershocks. The filled circles represent the largest aftershocks ( $>2.6$ ) occurring in a 24 hour period after the portable network had been installed, beginning at 00:00 UT of the 14 May 1990. The locations were made by the NZSO. The open circles, on the other hand, represent the reported locations of the mainshock and largest aftershocks up to 00:00 UT, 14 May 1990, a period of around 19 hours, determined using the National Network stations alone. The latter set of aftershocks appear to be consistently located between 5 and $15 \mathrm{~km}$ too far southeast. This was confirmed by choosing some of the later, better-located aftershocks, and relocating them, using HYPO-71 (Lee and Lahr, 1975) excluding the picks from the portable network stations. This procedure also showed the depths to be in error, the events being located generally between 2 and $12 \mathrm{~km}$ too deep. 


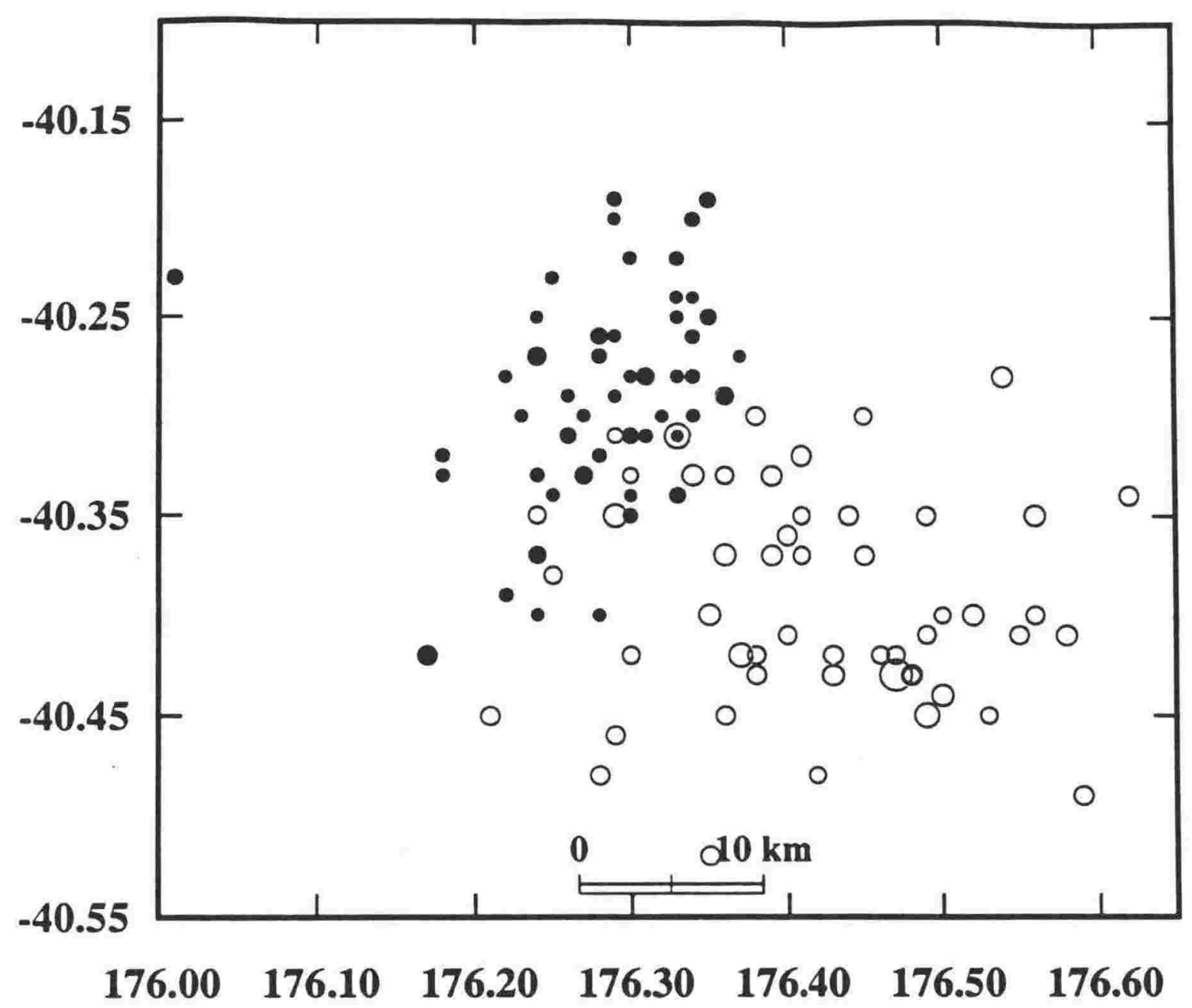

Fig. 2.3 Epicentres of the 13 May event aftershocks. Open circles - locations by National Network alone; closed circles - locations made using the portable micrearthquake network, together with the National Network stations. All locations are routine ones carried out by NZSO

Figure 2.4 shows the depth distribution of events occurring during the period 14-18 May 1990, these events being ones calculated with the inclusion of the portable network observations. There is a peak of activity at around $20 \mathrm{~km}$ depth, close to the presumed plate interface, and significant activity to depths as shallow as $10 \mathrm{~km}$. The activity falls off sharply below $20 \mathrm{~km}$. The small rise in the number of events between 25 and $36 \mathrm{~km}$ depth is to be associated with the ongoing aftershock sequence of the February earthquake, which is seen to be low in comparison with the shallower activity.

It is to be said that these locations were carried out by the Seismological Observatory using the standard procedure and standard velocity models. The Weber area happens to lie in the border region between the Wellington and Hawkes Bay regions, for which, different velocity models are defined. The Wellington standard model and the Hawkes Bay standard model are listed in Table 2.2. In such a case, the location procedure automatically computes a theoretical travel time which 


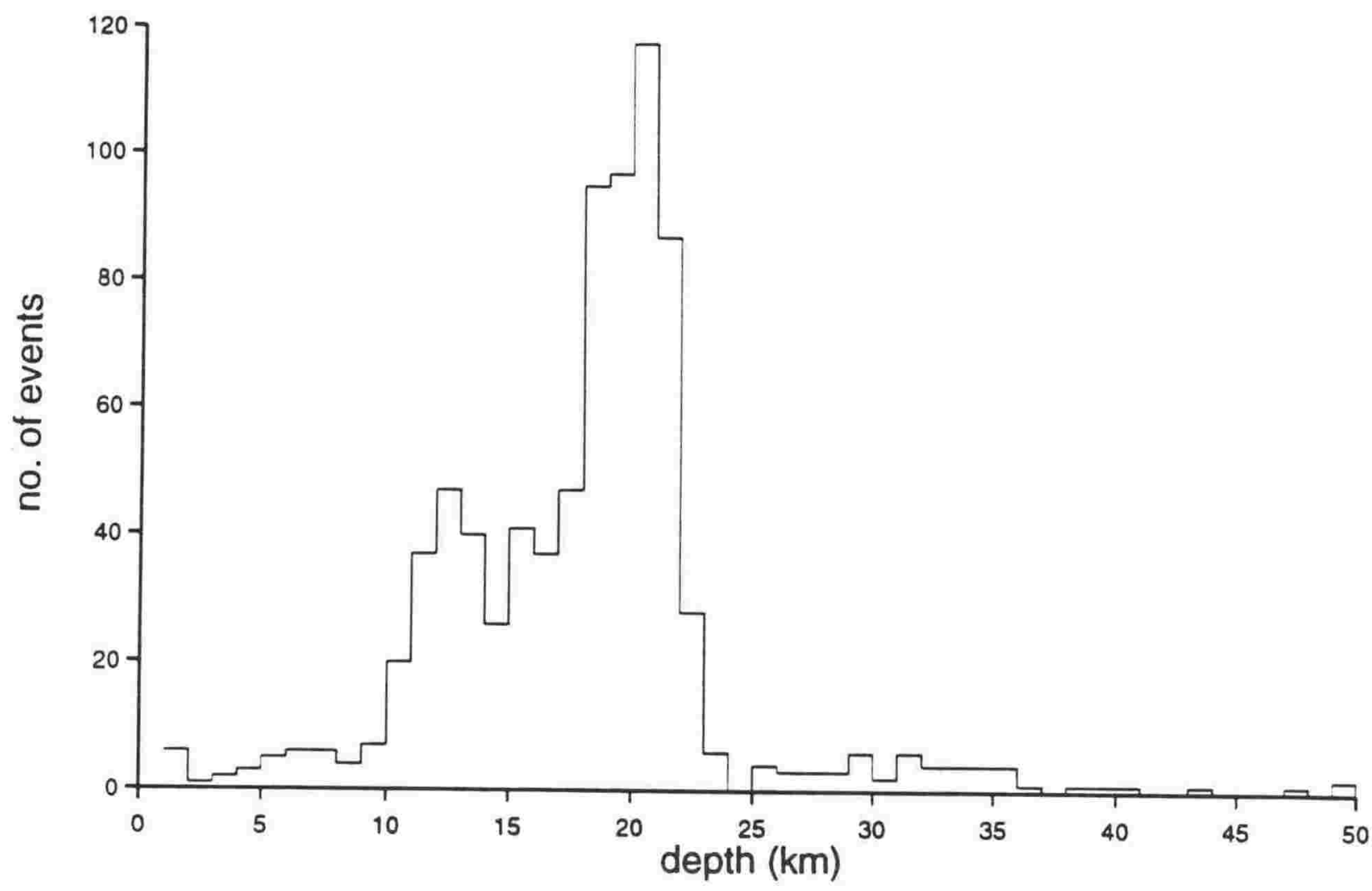

Fig.2.4 Depth distribution of the 13 May aftershocks in the period 14-18 May, when the microearthquake network was in place

is a weighted average of the travel times based on both velocity models, in such a way that there is a smooth transition as one moves across a boundary region.

Subsequently, further and more detailed analysis was carried out on all the Weber aftershock sequences by Robinson (1994) who performed a joint inversion of arrival times for hypocentres and velocity structure, thus arriving also at the best-fitting velocity model beneath the epicentral region. Robinson's final hypocentres from the February and May sequences together again cluster in two distinct depth ranges, almost exactly on top of each other, and with an approximately $4 \mathrm{~km}$ aseismic gap between them. The two groups of aftershocks lie in the depth ranges $5-18 \mathrm{~km}$ and $22-35 \mathrm{~km}$, somewhat shallower than the depths calculated by the routine inversions.

A knowledge of the focal mechanisms of the earthquakes in the two sequences is expected to reveal the general state of stress in the crust below Southern Hawkes Bay. The 13 May 
Table2.2a The Wellington standard velocity model

\begin{tabular}{|ccc|}
\hline $\begin{array}{c}\text { Depth to top } \\
\text { of layer } \\
(\mathrm{km})\end{array}$ & $\begin{array}{c}\text { P-wave } \\
\text { velocity } \\
(\mathrm{km} / \mathrm{s})\end{array}$ & $\begin{array}{c}\text { S-wave } \\
\text { velocity } \\
(\mathrm{km} / \mathrm{s})\end{array}$ \\
\hline 0.0 & 4.4 & 2.54 \\
0.4 & 5.63 & 3.16 \\
5.0 & 5.77 & 3.49 \\
15.0 & 6.39 & 3.50 \\
25.0 & 6.79 & 3.92 \\
35.0 & 8.07 & 4.80 \\
45.0 & 8.77 & 4.86 \\
\hline
\end{tabular}

Table2.2b The Hawkes Bay standard velocity model

\begin{tabular}{|ccc|}
\hline $\begin{array}{c}\text { Depth to top } \\
\text { of layer } \\
(\mathrm{km})\end{array}$ & $\begin{array}{c}\text { P-wave } \\
\text { velocity } \\
(\mathrm{km} / \mathrm{s})\end{array}$ & $\begin{array}{c}\text { S-wave } \\
\text { velocity } \\
(\mathrm{km} / \mathrm{s})\end{array}$ \\
\hline 0.0 & 5.5 & 3.3 \\
12.0 & 6.5 & 3.7 \\
33.0 & 8.1 & 4.6 \\
\hline
\end{tabular}

mainshock was large enough to yield a fairly good azimuthal coverage of first motions and has been interpreted as a thrust event in the overlying plate (Smith,1990). The aftershocks in the shallow group studied by Robinson have also revealed predominantly thrust mechanisms along a clearly defined fault-plane dipping $35^{\circ}$ to the north-west. Thrust events are common in this region (Reyners, 1989), and concur with the general direction of compression between the converging Australian and Pacific plates.

Also from Robinson's work, the deeper group of aftershocks lie on another clearly defined fault plane, steeply dipping at about $75^{\circ}$ to the north-west, and the composite fault plane solution is one of normal faulting, in agreement with the general state of tension within the upper $15 \mathrm{~km}$ of the bending, subducted plate. This is the same mechanism that had been found for the February mainshock (Smith, 1990). 


\subsubsection{Waveforms from the Weber Data Set}

From the data recorded on the L-network, a set of 35 events was chosen for further analysis, on the basis of the data quality, and the number of network stations triggered. Origin times and hypocentre information for these events are given in Table Al of Appendix A. In general, the waveforms from all aftershocks showed quite similar characteristics. Furthermore, the spacing of the L-network stations was close enough to give good coherence of waveforms throughout the array for many events, especially for the first onset of the signal. Figure 2.5 shows examples of aftershocks recorded on the L-network stations. The phases observed visually on these seismograms are typical of the majority of the aftershocks from the Weber event. An immediately visible feature of most of the seismograms, evident in Figure 2.5, is the difference in frequency content between different sections of the waveforms, particularly in the P-wave and Pcoda. The first $4 \mathrm{~s}$, approximately, of the seismograms contain frequencies which are clearly higher than those in the rest of the P-coda, leading to the suggestion that these sections of the signal are associated with propagation through different parts of the lithosphere. The frequencies contained in this first signal vary from station to station and from event to event. The amplitude of this signal also varies. In several cases it is quite strong and has the form of a "pulse" of limited duration ( $1-2 s)$. It appears to consist of a complex, closely-spaced group of sharp arrivals. In other cases, the amplitude in these first $4 \mathrm{~s}$ is low, relative to the next major arrival, and no dominant "pulse-like" phase is seen.

A prominent second group, about $4 \mathrm{~s}$ after the initial onset, is present on almost all aftershocks. It has conspicuously lower frequencies than the first $\mathrm{P}$ arrival, and generally larger amplitude. Within this second arrival, several sharp phases, traceable through the L-network, may often be distinguished. Further reference to these features is made in Chapter 5. The shallow depths of the aftershocks meant that the whole of the P-coda was in general complex and reverbatory, although the amplitude of the $4 \mathrm{~s}$ arrival is clearly dominant.

Further enlargement of the initial portion of the signal reveals that the high-frequency group is often preceeded by a small-amplitude, lower-frequency onset, which takes the form of a single, simple pulse, followed $0.5-1.5 \mathrm{~s}$ later by the usually impulsive onset of the higher frequency energy. The initial pulse has been interpreted as the first-arriving $\mathrm{P}_{n}$. The small station spacing of the L-network meant that, where the noise level was low enough, good coherence was observed for this first onset, as well as, although to a lesser extent, for the onset of the higher frequency signal, over most of the network. Some examples in which the phases discussed are particularly clear are seen in Figure 2.6, in the form of array sections, reduced at a velocity of 8.0 $\mathrm{km} / \mathrm{s}$.

The frequency characteristics of the seismograms were better displayed by applying frequency-time analysis (FTAN) to the relevant portions of the signals. The method used was a slight modification of the original multiple-filtering technique of Dziewonski et al (1969) for surface wave dispersion calculations. A flow chart and brief description of the technique is given 

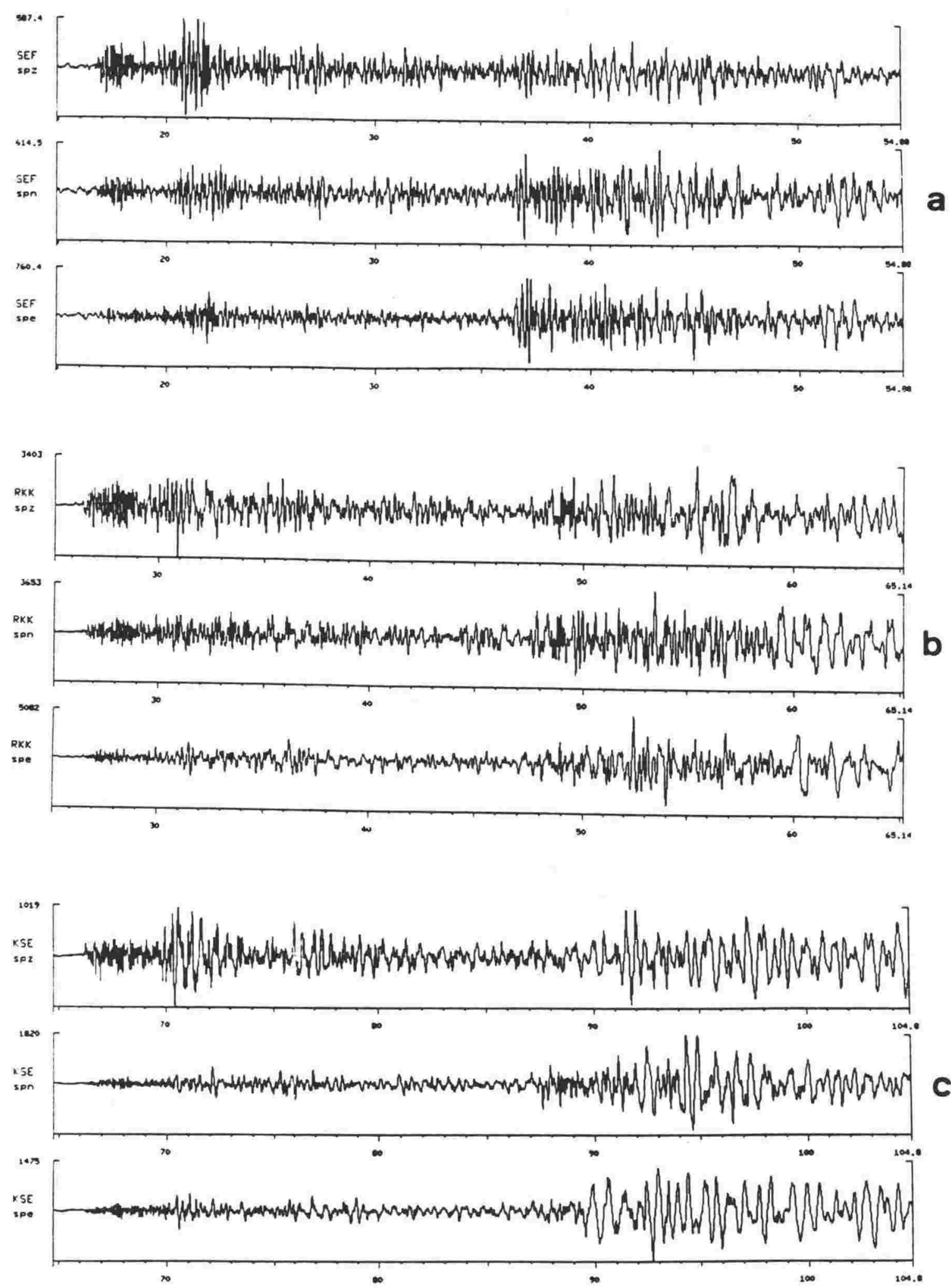

Fig. 2.5 3-component seismograms of Weber aftershocks recorded on L-network stations.

(a) Event 900513.0511

(b) Event 900513.0459

(c) Event 900513.0637 
in Appendix B. The basic program used was that described by Burton and Blamey (1972). The technique essentially decomposes a given time series into a "map" representing the instantaneous amplitudes of the signal as a function of frequency and group velocity. The program was here modified to express the amplitudes at equal steps of arrival time rather than of group velocity.

A few examples of the results of this technique are shown in Figures $2.7 \mathrm{a}-\mathrm{e}$, with the time axes coinciding with those of the signals for easy comparison. The dark areas of the diagrams correspond to high instantaneous amplitudes, and the output matrices have been scaled to a maximum of $99 \mathrm{~dB}$. In Figures $2.7 \mathrm{a}-\mathrm{d}$, the first $16 \mathrm{~s}$ of the $\mathrm{P}$ and P-coda are shown. The start of the signal is clearly evident by the end of the "white" area, representing the background noise, in the first $2 \mathrm{~s}$, or so, of the seismograms. The presence of isolated dark areas in all the diagrams is indicative of the presence of "modes" arriving at the receiver, the different frequency contents suggesting propagation through different parts of the lithosphere, and/or different content of Swave. The high frequencies in the first phase of the signal appear as isolated energy maxima, of differing intensity. Figure $2.7 \mathrm{~b}$ shows an especially intense energy arrival of dominant frequency approximately $10 \mathrm{~Hz}$. In Figure $2.7 \mathrm{c}$, the dominant frequency for this phase is around $12.5 \mathrm{~Hz}$, while in Figure 2.7d, there is a suggestion of a frequency content up to $15 \mathrm{~Hz}$. The second energy arrival group, about $4 \mathrm{~s}$ after the initial onset is normally apparent as the most intense dark area on the diagrams, its frequency content varying between 2 and $8 \mathrm{~Hz}$. It often appears to contain 2 separate, simultaneously arriving "modes", as in Figures $2.7 \mathrm{c}$ and $2.7 \mathrm{~d}$. A second low-frequency arrival is also recognisable on most diagrams at about $8-11 \mathrm{~s}$ after the first onset. Note that very little of the low-frequency energy arrives before the $4 \mathrm{~s}$ onset, while there are none of the highest frequencies in the later part of the P-coda. Sometimes there appears to be a repetition of the highfrequency pattern after a few seconds (see e.g. Figure $2.7 \mathrm{~d}$ ), perhaps representing a near-source reflection and re-transmission through the same wavepath.

Figure $2.7 \mathrm{e}$ shows a longer time sample, including the S-wave, for the same event as in Figure $2.7 \mathrm{~d}$. The S-wave arrival is quite clear, with dominant frequencies of around $2.0 \mathrm{~Hz}$ for the first 10 s of the S signal. An isolated higher frequency arrival (at $35 \mathrm{~s}, 7.0 \mathrm{~Hz}$ ) seems to mirror the pattern of P-wave behaviour in the first few seconds of the seismogram. For a given event, there may be a variation in frequency content between different stations of the L-network. This is believed to be due to geological conditions at the seismograph site. Even though all stations are located on the same rock type, Mesozoic greywacke, there may be differences in the state of fracture within the rock which affect the frequency response. For example, the site at NDE appears to be inefficient at recording the high frequencies as can be seen at a glance from Figure 2.6, while SEF and PDD seem to reproduce them well. Instrument response is unlikely to be the reason for these differences since all instruments have similar response characteristics.

The frequency characteristics are also evident in the frequency spectra of consecutive time windows of the seismograms. Some examples are displayed in Figure 2.8. The amplitude spectra have been computed for the first $3-4 s$ of the P-wavetrain, containing the high frequency group, 
and for the subsequent 4-5s, containing the higher amplitudes. It is clear from the spectra that the high frequencies $(>10 \mathrm{~Hz})$ are almost invariably confined to the first portion of the signal, often occurring as narrow peaks in the spectrum. The second time window generally shows peaks in the 3 - $5 \mathrm{~Hz}$ range. For each selected event, all the spectra from available L-network records are displayed. The absence of high frequencies, even in the

\subsubsection{Other Events Recorded on the L-Network}

During the period of operation of the L-network, a large number of events were recorded from all over New Zealand. Of these events, some were selected to provide additional estimates of along-strike P-wave velocity in the subducted slab. The events chosen were those in the Southern Hawkes Bay area, and a set in the Lake Tennyson area of the South Island, which were more than $100 \mathrm{~km}$ from Wellington. Figure 2.9 shows the epicentres, as located routinely by the NZSO, of events in these regions of interest, a selection of which were then used for further study. The event identification numbers and source parameters are listed in Tables A2 and A3 of Appendix A.

During this period, a further group of events from the Weber region were recorded, as seen in Figure 2.9, representing the ongoing aftershock sequence of the 19 February earthquake. As can be seen from Table A2, these events were generally deeper than the ones selected in the Weber data set. The waveforms from these events were also rather different from the ones in the Weber data set, with the main energy arrivals having different characteristics than those discussed in the previous section. An example is shown in Figure 2.10a.

Another cluster of earthquakes during the same period was that in the South island, shown in Figure 2.9. These events were also part of an aftershock sequence, that of a magnitude 6.0 earthquake which had occurred at Lake Tennyson on the 10 February 1990. These aftershocks were all shallow, most of their depths being restricted to $5 \mathrm{~km}$ in the NZSO location procedure. The waveforms from these events also showed a general similarity, with a high-frequency first phase, followed closely after by a dominant group. An example is shown in Figure $2.10 \mathrm{~b}$. The other events occurring in the South Island were either too close to Wellington, or else very deep, occurring where the subducted slab has already begun to dip steeply. 

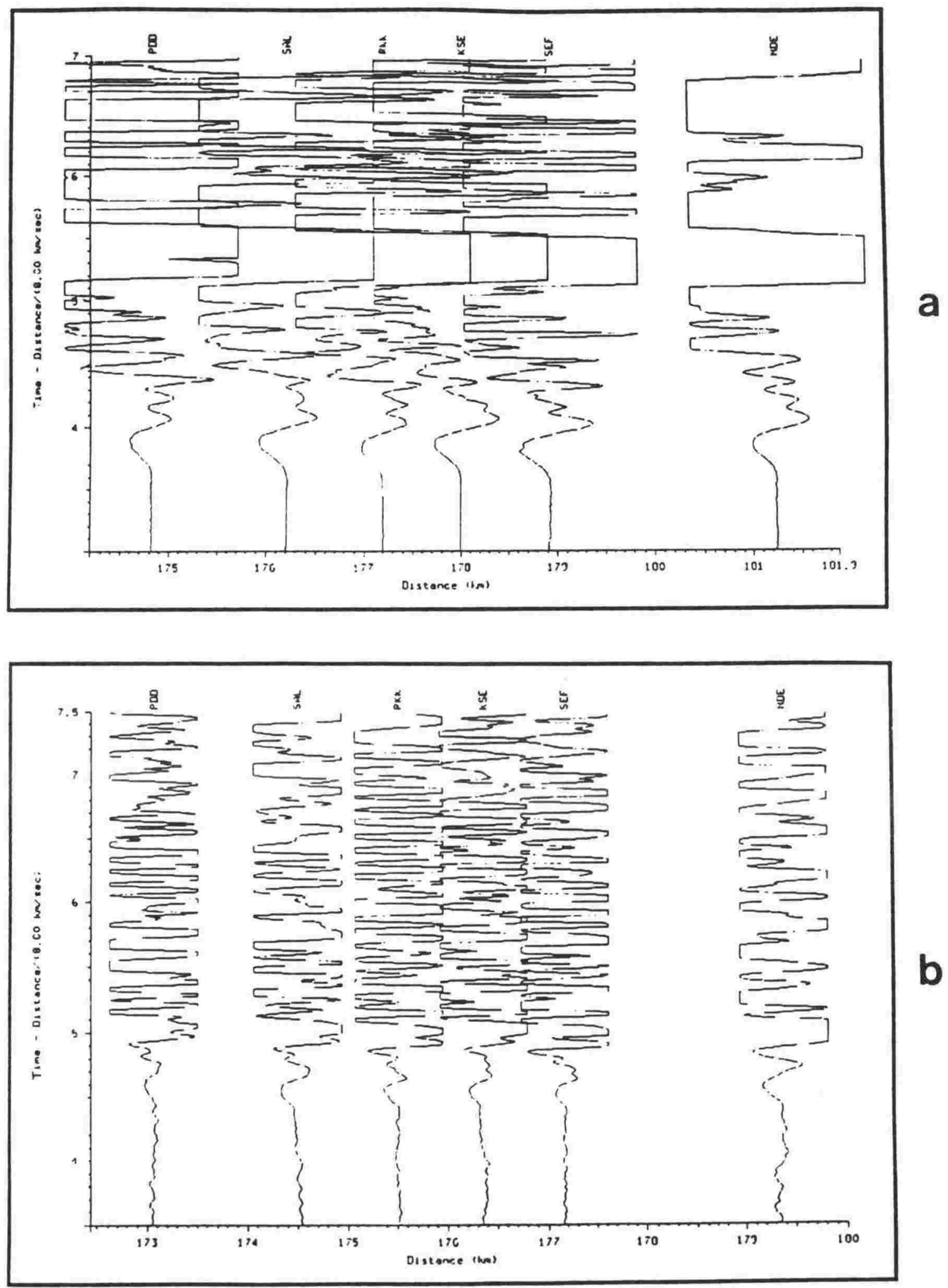

Fig. 2.6 Array sections of some of the Weber data recorded across the L-network, shown at high vertical amplification to clarify the first onset. Reducing velocity is $8.0 \mathrm{~km} / \mathrm{s}$ in all cases.

(a) Event 900513.0423 (mainshock)

(b) Event 900513.0459 

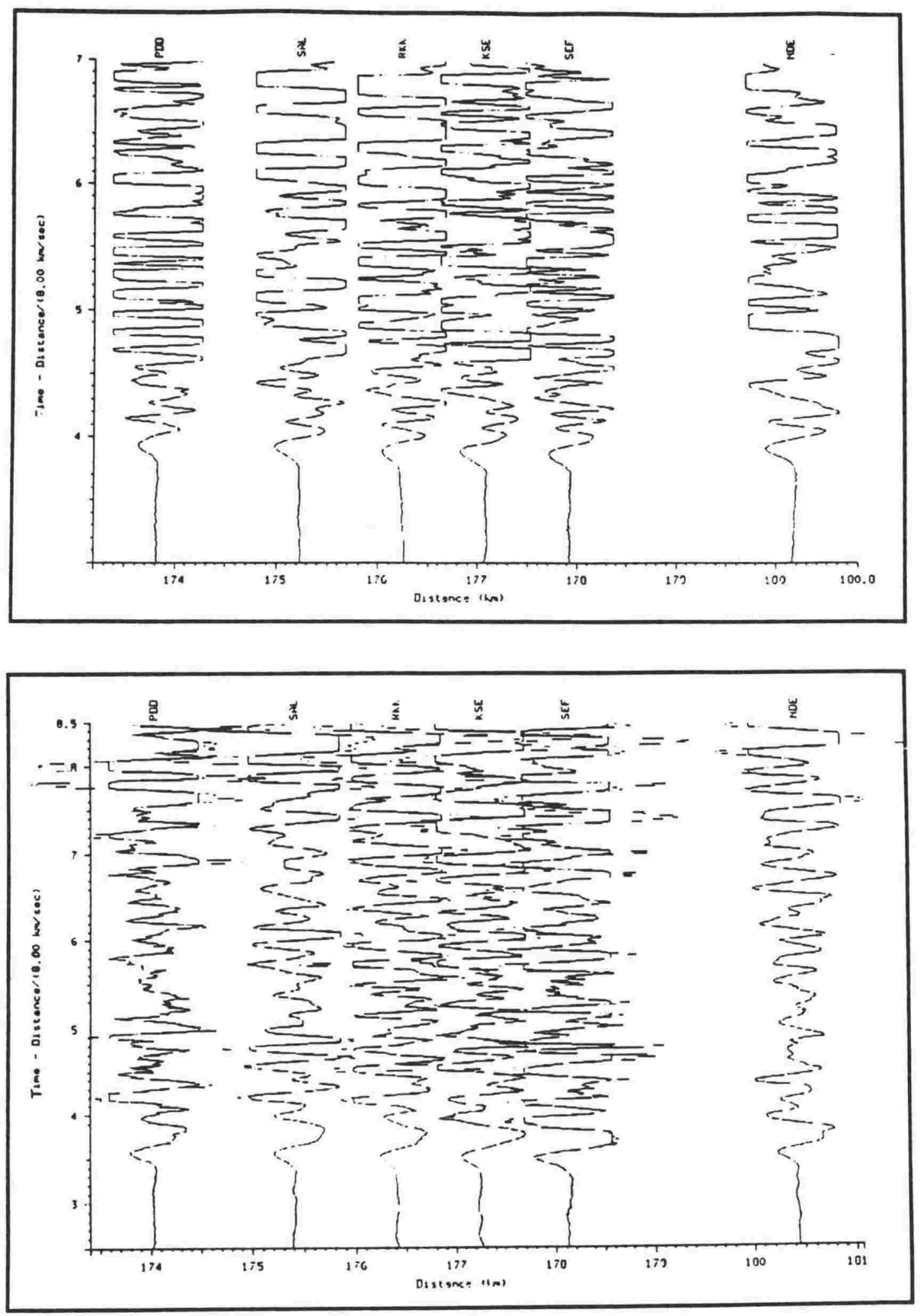

Fig. 2. 6(cont.)

(c) Event 900513.0541

(d) Event 900513.0711 


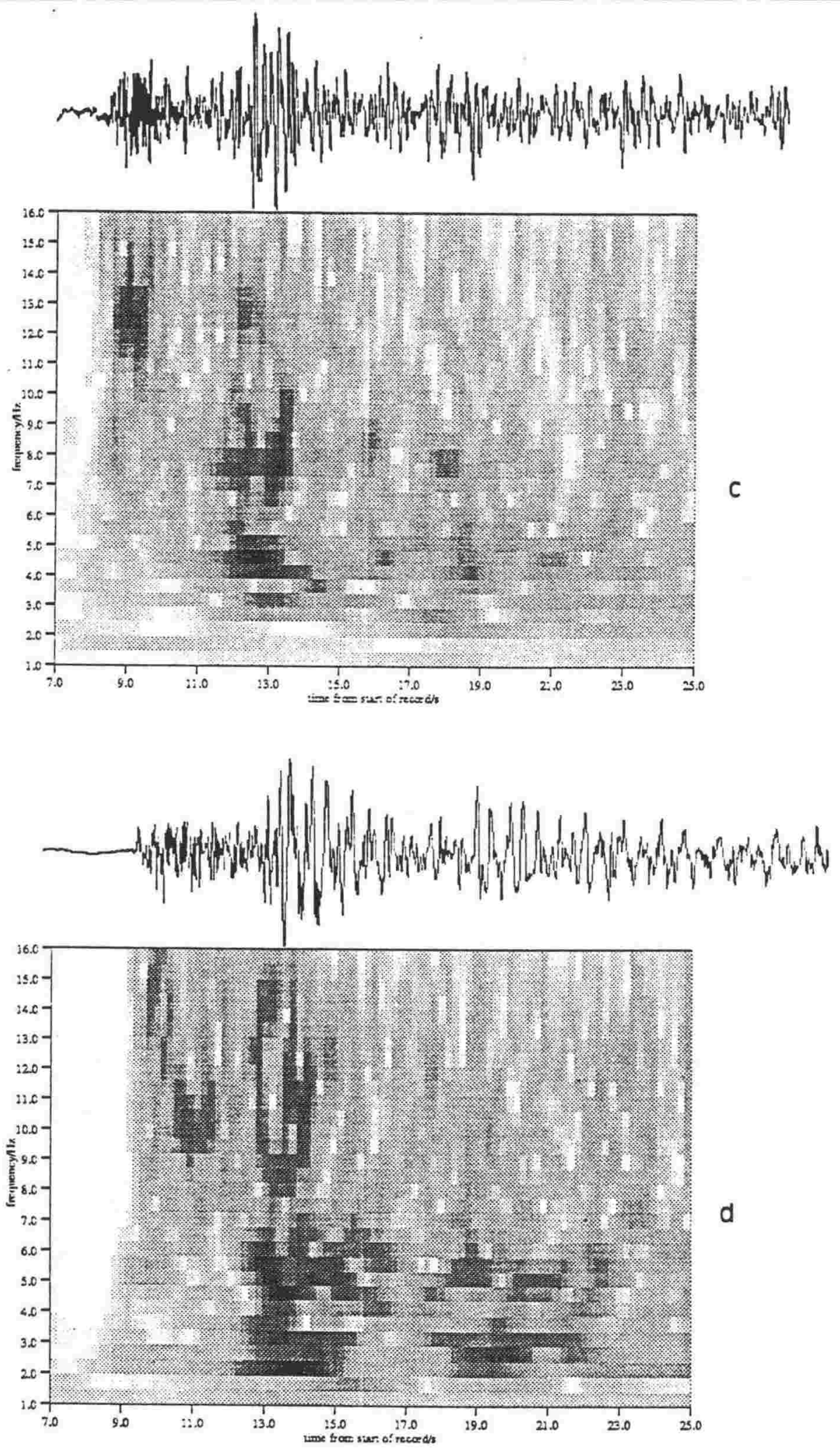

Fig. 2. 7(cont.)

(c) Event 900513.0511 at SEF

(d) Event 900513.0637 at KSE 


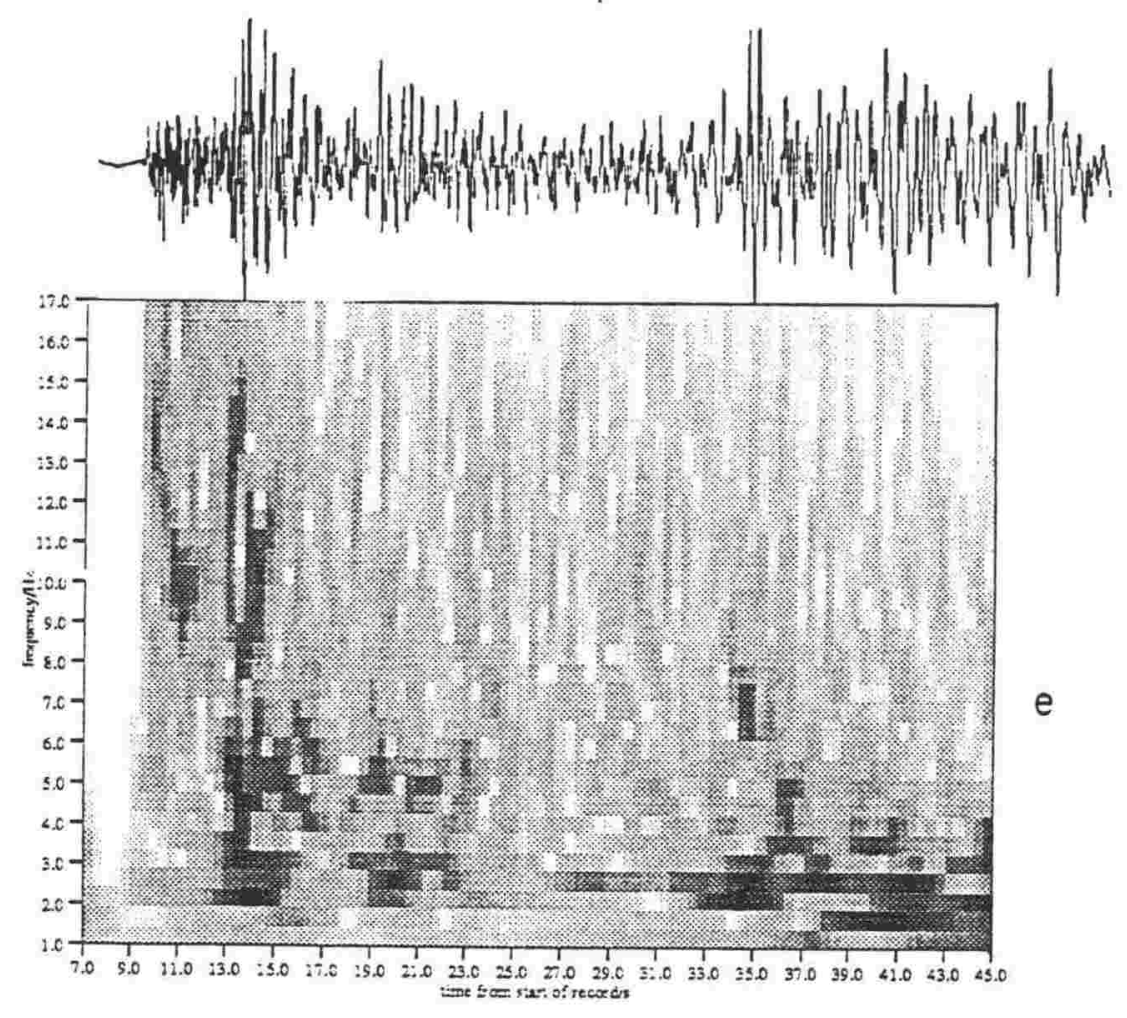

Fig. 2.7(cont.) (e) Longer time-sample FTAN diagram, including S-wave, for event 900513.0637 at KSE.

\subsection{Inversion of Arrival Times for Wavefront Velocity}

In this chapter, we shall restrict ourselves to the estimation, as accurately as possible, of the apparent velocity of the very first phase in the seismograms. The distances involved are such that this phase can be assumed to be a refracted arrival. The nature of later phases on the seismograms will be dealt with in a later chapter.

The availability of network arrival times from a number of earthquakes of similar origin made it possible to obtain an estimate of the wavefront apparent velocity corresponding to the first arrival, by using a least-squares inversion method. A small-aperture array such as the Lnetwork has the advantage that it may be considered as a "point" along the wavefront. This could be assumed to be the case here, where the epicentral distance was approximately $175 \mathrm{~km}$, while the linear dimension of the network was about $3 \mathrm{~km}$. Waves arriving at different stations of the network may be assumed to have travelled exactly the same path. A small array, however, has the disadvantage that, since the stations are so close (in this case about $1.1 \mathrm{~km}$ ), the arrival times at adjacent stations vary by only a fraction of a second, and therefore the calculation becomes 


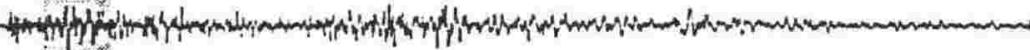

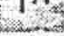
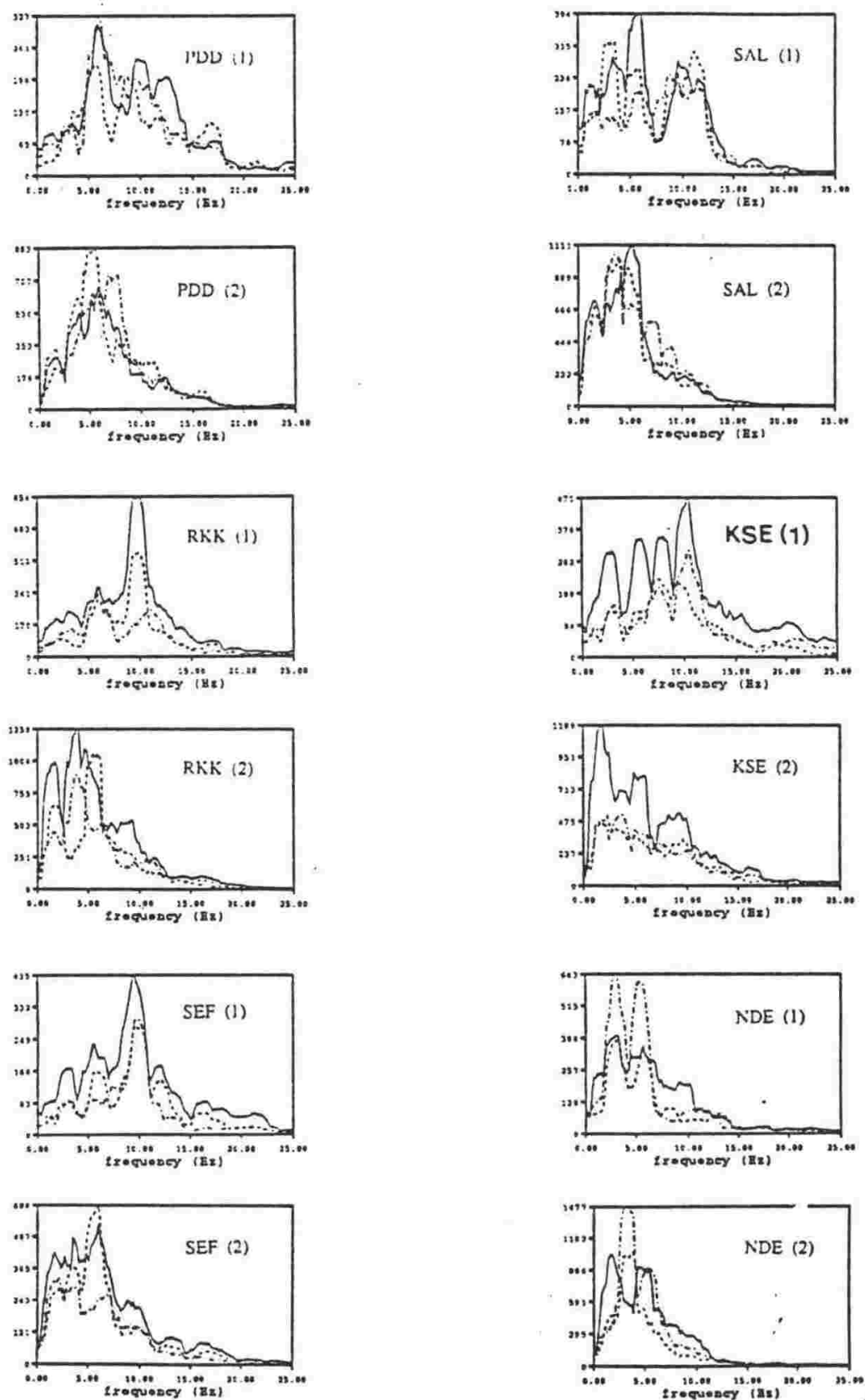

Fig. 2.8 Amplitude spectra for (1) the first 3s, and (2) the subsequent $5 s$, of event 900513.0459, recorded on all 6 operative stations. The seismograms shown are vertical component. In the spectra figures, the dashed curves are the spectra for the horizontal components 


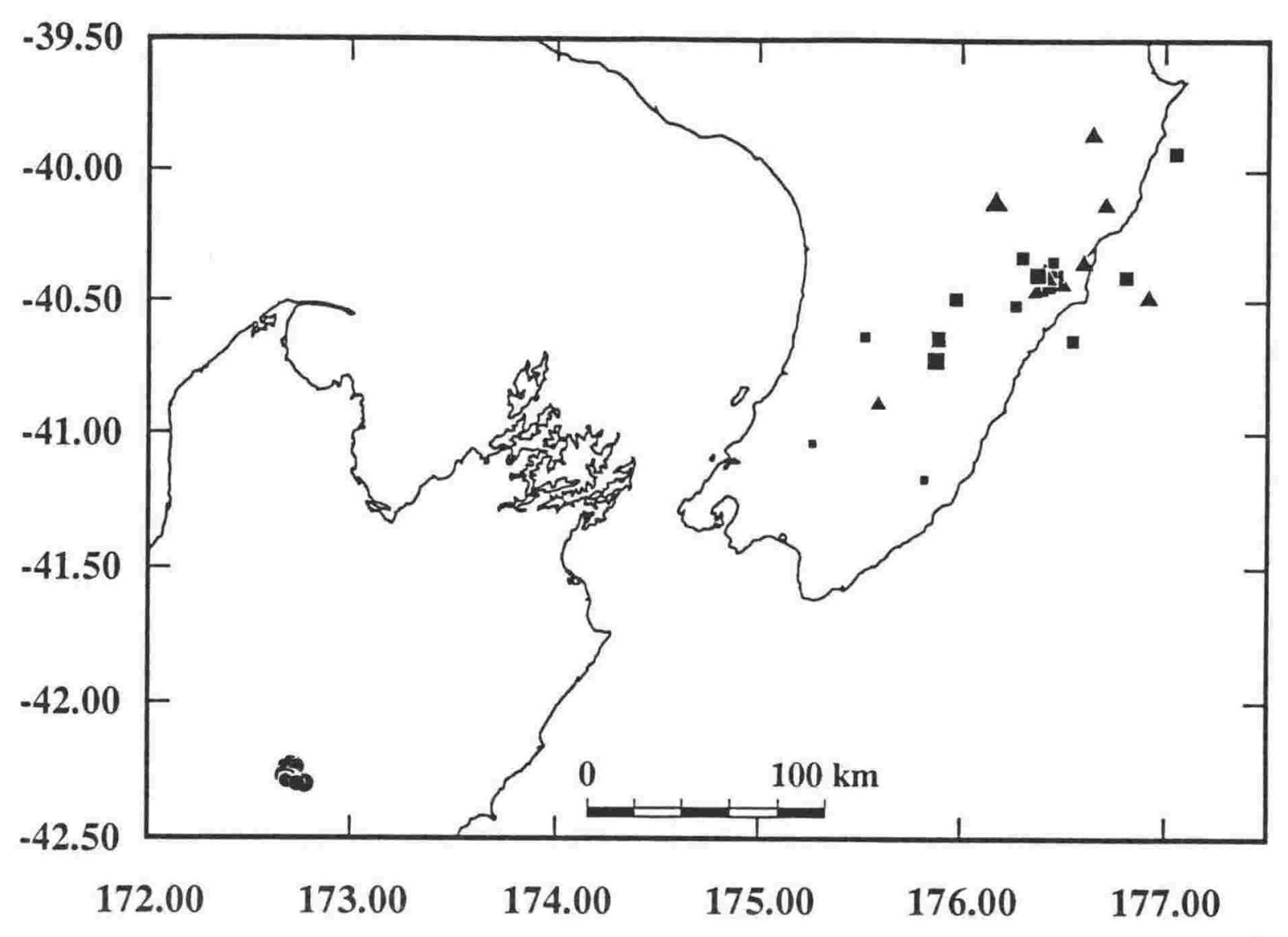

Fig. 2.9 Events in S. Hawkes Bay and the Lake Tennyson area that were recorded on the L-network besides the Weber aftershock sequence. Size of symbols represents magnitude, ranging from 2.0 to 4.8 (see Tables A.2 and A.3)

very sensitive to errors in pick times. This consideration was one of prime importance, and the best compromise was to use only those events of the highest quality, having a high signal-to-noise ratio at the start of the signal. Moreover, the events chosen had to have a coherent first pulse throughout the network.

Another problem arose with events which were of high quality according to the abovementioned criteria, but which either, for some reason, did not trigger all the stations in the network, or gave an unreliable pick at one of the stations. If the "unusable" station was one of the intermediate ones, such as SAL, RKK, SEF or EGB, this did not affect the inversion too seriously. However, if the missing station was one of the end stations PDD or NDE, then the geometry of the array departed appreciably from the ideal L-shape, and the reliability of the inversions suffered. This was especially a problem since EGB was inoperative throughout the May 13 sequence, and therefore if NDE was missing the array would be reduced to almost a single-line leg, and the azimuthal control of the wavefront solution would suffer drastically. Station PDD 


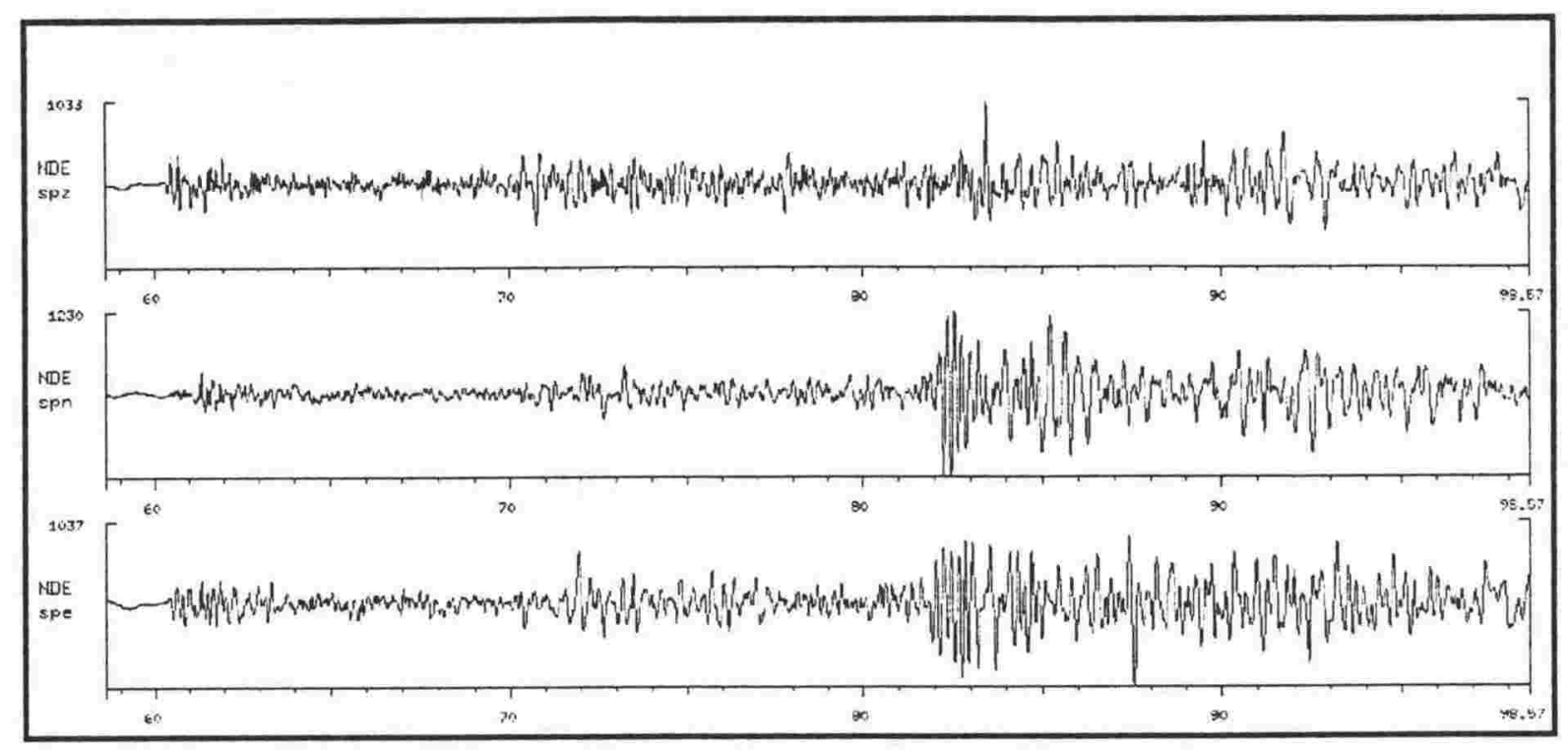

Fig. 2.10a 3-component seismogram recorded at station NDE of the L-network, from an event occurring in the Weber area on the 21 April 1990 (event no. 137). The event was reported to be $44.1 \mathrm{~km}$ deep. Note the difference in appearance of the waveforms compared with those of the shallow, 13 May 1990 aftershocks (Figure 2.5).

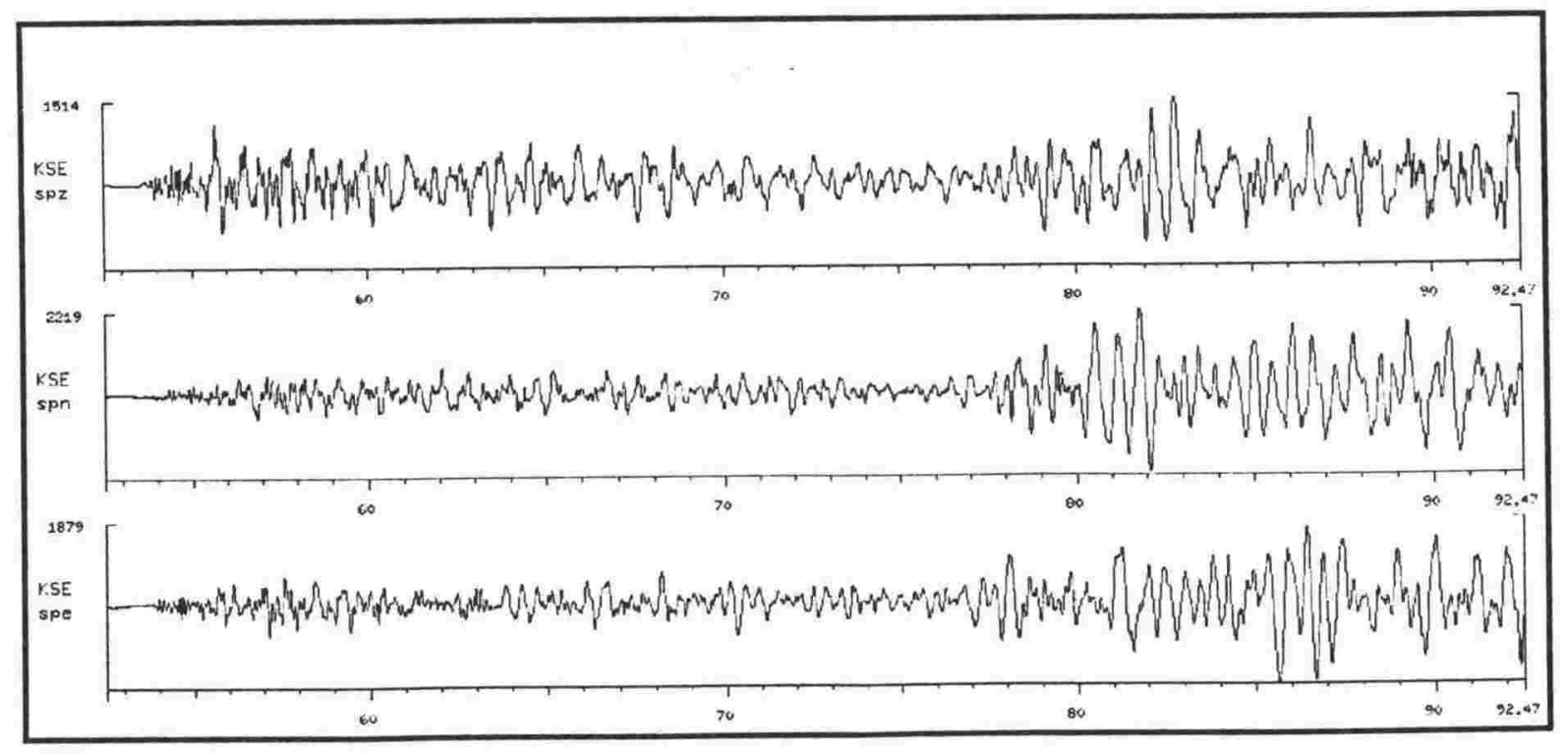

Fig. 2.10b 3-component seismogram recorded at station KSE of the L-network of an event occurring in the Lake Tennyson area, S. Island, on the 29 March 1990 (event no. 304). The event was part of an aftershock sequence at very shallow depth in the crust (depth restricted to $5.0 \mathrm{~km}$ in the NZSO solution). The general waveform features are similar to those of the Weber data set. 
also caused some problems in this respect since it was often contaminated with a high frequency noise which made picking of the first arrival too difficult. Events that presented these problems were not used.

Fortunately, the large amount of data available ensured that a number of events that satisfied the criteria could be found. The selection process left 15 events which were of high enough quality for their array inversion to be deemed reliable.

Picks of the first arrival were only made for the vertical component. The procedure was carried out by viewing the data on the seismic analysis package xpick (Figure 2.11) which allows enlargement of the traces to any extent along both horizontal and vertical scales. Since, for the purpose of apparent velocity calculations, only the delay times between stations were of interest, the arrival times were measured at the first peak or trough of the signal, rather than its onset, as this could be done with better precision. By this method, and for the chosen events, the picking accuracy was at least $\pm 0.02 \mathrm{~s}$. The seismograph absolute timing accuracy was $\pm 0.01 \mathrm{~s}$.

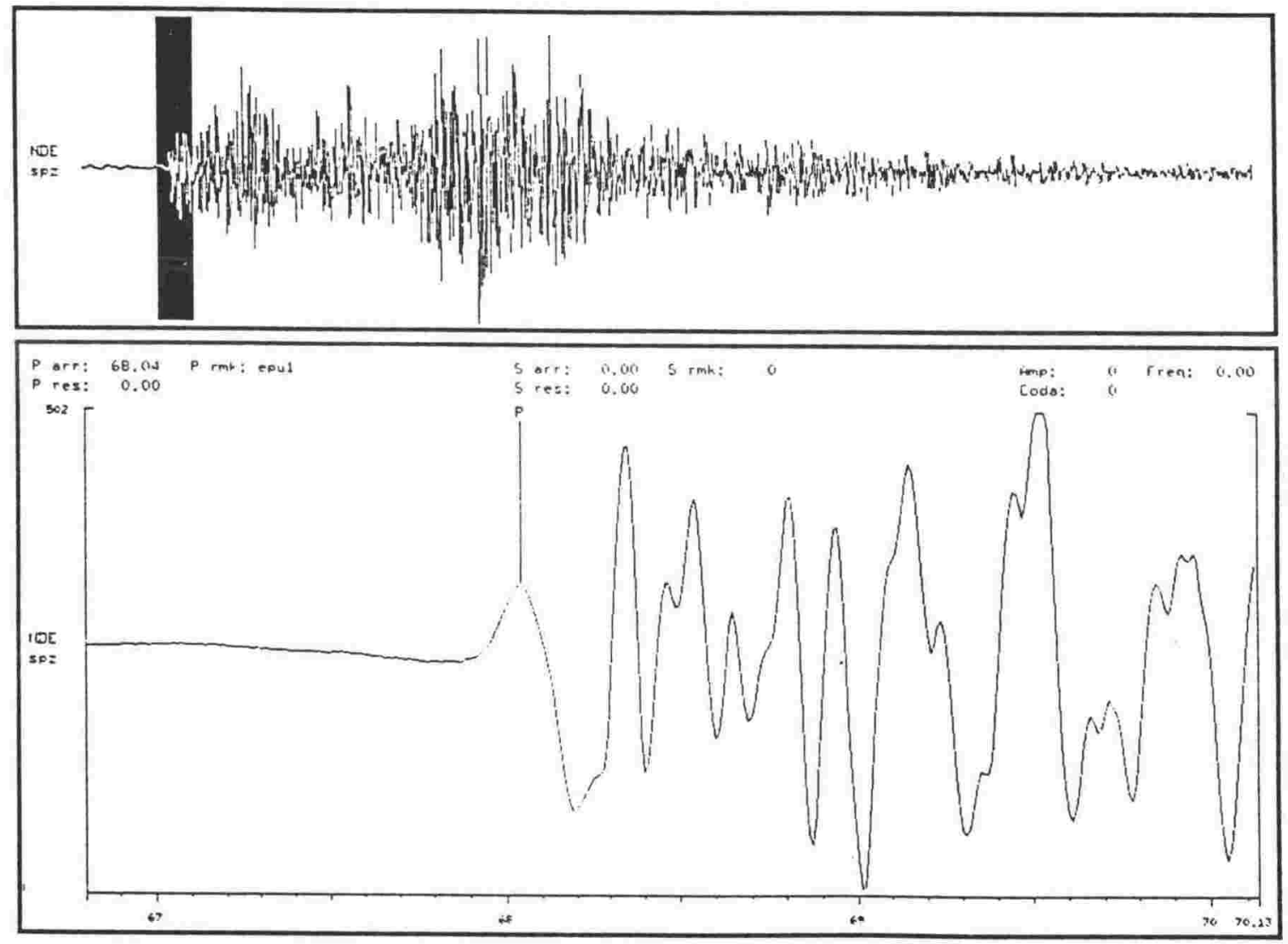

Fig. 2.11 An example of the picking window in the xpick programme 
The method of estimation of wavefront velocity (which will be taken to mean both speed and azimuth) was as follows:

Consider an array of $m$ stations, having coordinates $\left(x_{1}, y_{1}\right), \ldots\left(x_{m}, y_{m}\right)$, and an arbitrary reference point outside the array, for simplicity having coordinates $(0,0)$ (Figure 2.12 ). Let the arrival time at this station be $t_{o}$, and the observed arrival time at the $i$-th station be $t_{i}$. Then

$$
t_{i}=t_{0}+\mathbf{p} \cdot \mathbf{r}+\tau_{i}
$$

where $\mathbf{p}$ is the wavefront slowness, $\mathbf{r}$ the distance vector to the station, and $\tau_{i}$ is the time residual at that station.

For $m$ observations at $m$ array stations, we may write

$$
\begin{gathered}
t_{1}=t_{0}+\frac{\sin \phi}{c} x_{1}+\frac{\cos \phi}{c} y_{1}+\tau_{1} \\
: \\
t_{m}=t_{0}+\frac{\sin \phi}{c} x_{m}+\frac{\cos \phi}{c} y_{m}+\tau_{m}
\end{gathered}
$$

where $x_{i}, y_{i}$ may here be taken as the absolute values of the corresponding distances, $c$ is the wavefront speed and $\phi$ the azimuth (measured from North). Provided there are more than 3 observations, this system of equations may be solved by a least-squares inversion for the quantities $t_{0}, \frac{\sin \phi}{c}, \frac{\cos \phi}{c}$ which minimise the norm of the residual vector $\left(\tau_{1}, \ldots, \tau_{m}\right)^{T}$. The speed and azimuth are then easily found from the last 2 quantities.

In the case of the Weber data set, and the Lake Tennyson data set, the events being dealt with were all coming from a relatively small source region, and the epicentral distances were large enough that the variation in azimuth for different events was not more than $5^{\circ}$ at Wellington. It was then possible to carry out another inversion using the "global" data set, and solving for one best-fitting pair of wave-speed and azimuth. For $p$ events and $m$ observations per event, the system of equations now becomes equivalent to an $(m p \times(p+2))$ matrix to be solved for the $(p+2)$-vector

$$
\left(t_{0}^{1}, \ldots, t_{0}^{p}, \frac{\sin \phi}{c}, \frac{\cos \phi}{c}\right)^{T}
$$

The solution of the least-squares problem was by the method of Householder transformations as described in Lawson and Hanson (1974).

Picks which were of good quality but not used in the individual event inversions due to insufficient available picks for that event, were used in the global inversions provided there were at least three picks for that event. 


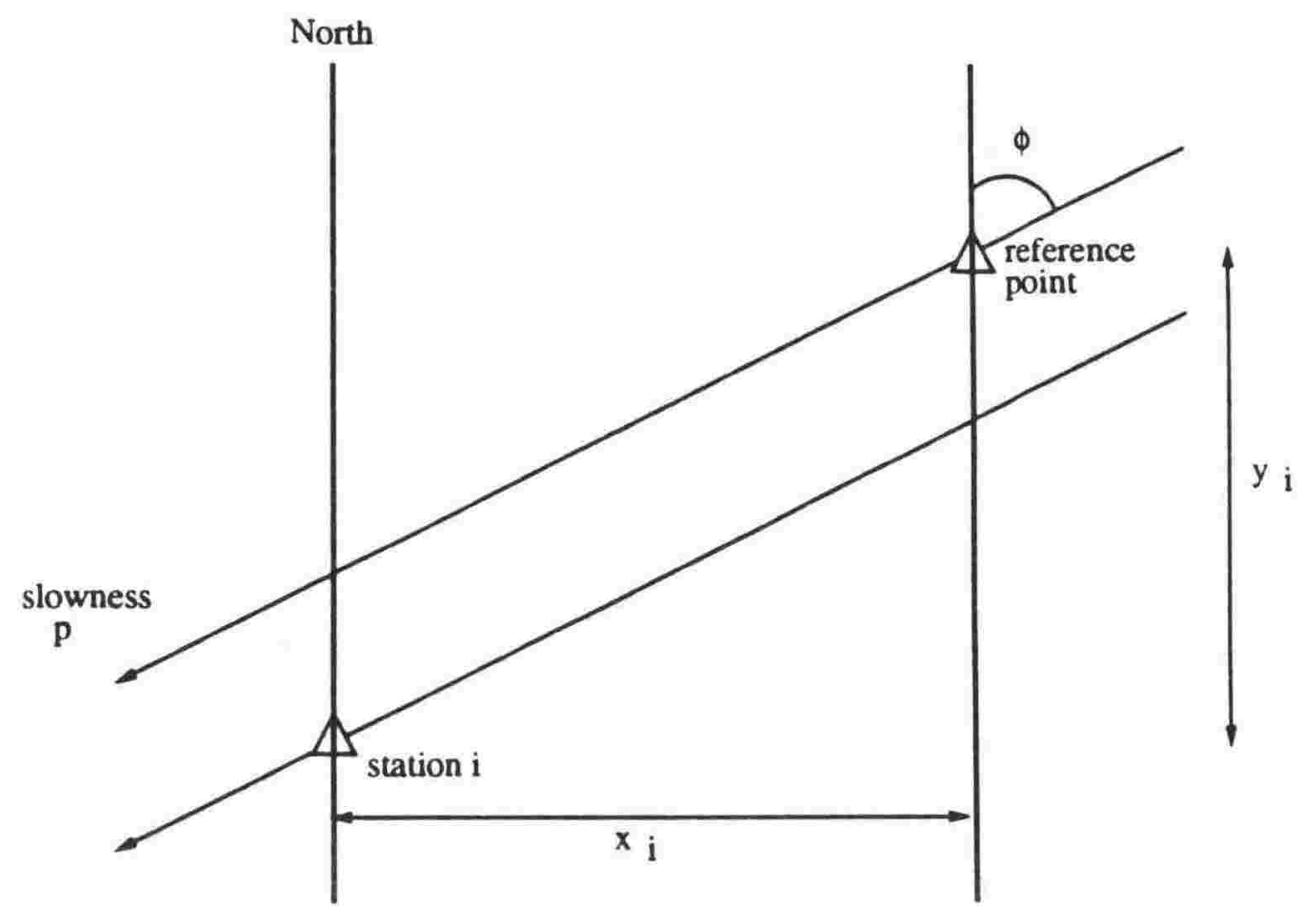

Fig. 2.12 A plane wavefront incident on an array of seismographs

The small arrival time differences meant that station delay terms would be significant, particularly since the network was sited on hilly terrain and there were considerable differences in elevation between stations. The maximum difference in height between any two stations was about $300 \mathrm{~m}$. Since all stations were on the same rock type, namely greywacke, it was possible to apply a correction for elevation to the individual arrival times. A P-velocity of $4.5 \mathrm{~km} / \mathrm{s}$ in the top few kilometres was assumed (Garrick, 1968). The station delays corrected for are shown in Table 2.3 .

Using the same procedure, it was decided to carry out separate calculations of the wavefront velocity using arrival times at an extended network consisting of the L-network and a few stations of the Wellington network. The stations chosen were WEL, CAW and MRW, since their location was such that the wavefront would be subject to a similar travel path geometry as for the L- 
network. Still, the result from the extended network was not expected to be as true a representation of the wavefront velocity as that from the much smaller L-network. The inclusion of the extra stations helped in that it increased the time difference across the whole network from about $1.1 \mathrm{~s}$ to about $4.6 \mathrm{~s}$, so that the inversion was much less sensitive to picking errors. Pick times for CAW and MRW were not read first-hand but were taken from the NZSO pick files, so that in this case, the arrival times at the initial onset had to be used. (A look at some printed waveforms from these two stations confirmed that the same phase was being picked as on the Lnetwork stations, judging from the great similarity of the onsets). Use of the extended network was made only for the Weber data set. The elevation corrections for the three extra stations are included in Table 2.3 .

\subsection{Results}

Tables $2.4 \mathrm{a}-\mathrm{d}$ list the selected events and contain results from the various inversions. The event identification numbers are found in Appendix A. $\phi_{e}$ is the azimuth of an epicentre from the network (measured at KSE) using the epicentral coordinates reported in the NZSO listing; $\phi$ is the calculated azimuth of the incident wavefront, as given by the least-squares inversion. The

Table2.3 Delay corrections applied to L-network stations and the three Wellington network stations used in the inversion

\begin{tabular}{|lc|}
\hline Station & $\begin{array}{c}\text { Delay } \\
(\mathrm{s})\end{array}$ \\
\hline PDD & 0.02 \\
SAL & 0.03 \\
RKK & 0.00 \\
KSE & 0.00 \\
SEF & 0.00 \\
EGB & 0.05 \\
NDE & 0.06 \\
& \\
WEL & 0.00 \\
CAW & 0.05 \\
MRW & 0.03 \\
\hline
\end{tabular}


difference between these two azimuths will be referred to as the azimuthal anomaly $\delta \phi . c$ is the measured wavefront speed. The station residuals from the global inversion were grouped by station, and their means and standard deviations are shown in the corresponding tables.

The different kinds of inversion involve errors of different significance. When using the Lnetwork alone, the maximum inaccuracy in reading the peak of the first wavelet from the seismogram was $\pm 0.02 \mathrm{~s}$. A few test inversions showed that, with this accuracy (and neglecting timing errors) the wavefront speed could be determined to $\pm 0.2 \mathrm{~km} / \mathrm{s}$, and the azimuth to $\pm 2.5^{\circ}$. When including the stations WEL, MRW, CAW, the picking errors will of course be less significant. The errors here are a measure of the reliability of the inversion by looking at the effect of removing one station at a time.

\subsubsection{The Weber Data Set}

The wavefront speeds given by the inversions are consistently high. The average of around $8.7 \mathrm{~m} / \mathrm{s}$ for the Weber data set, using the L-network alone, is unusually high compared with average upper mantle P-velocities, especially when considering that the path from Weber to Wellington is slightly downdip, and would therefore yield an apparent velocity smaller than the refractor velocity. The quantification of this effect in the case of a dipping slab is fully discussed in the next chapter.

In Table $2.4 \mathrm{a}$, the wavefront azimuths $\phi$ are seen to be, on average, $2^{\circ}$ larger than the actual azimuth of the epicentres relative to the network. In other words, the wavefront approaches from a more easterly direction than expected. The azimuthal anomaly $\delta \phi$ has not been included in the tables because of the uncertainty in $\phi_{e}$ arising from the mislocation of the epicentres. As discussed in Section 2.3.1 this mislocation may lie between 5 and $20 \mathrm{~km}$, which would imply that $\phi_{e}$ is consistently between about 1 and $5^{\circ}$ smaller than that given in Tables $2.4 \mathrm{a}$ and $2.4 \mathrm{~b}$. Rather than calculate the individual $\delta \phi$, which would contain a large, and unmeasurable error, a more realistic estimate of the azimuthal anomaly was made by using an "average epicentre" of $40.3 \mathrm{~S}$, 176.3E, estimated from the epicentres located with the micro-earthquake network. As can be seen from Figure 2.3, the accurately located epicentres are far less scattered, and this is a reasonable value. The azimuth of such an epicentre would be $50^{\circ}$, and it is seen that all of the wavefronts in Table 2.4a are now consistently eastwards of the expected azimuth. If we use the average wavefront azimuth of $55.7 \pm 3^{\circ}$, we may state that the average azimuthal anomaly for the Weber data set is $5.7 \pm 3^{\circ}$. This effect is intuitively attributed to the fact that the first arrival follows a refracting horizon within the dipping subducted slab, and is therefore laterally refracted off the slab. This is also fully discussed in the next chapter. 
Table2. 4a Inversion results from the Weber data set, using the L-network

\begin{tabular}{|c|c|c|c|}
\hline $\begin{array}{l}\text { Event } \\
\text { I.D. }\end{array}$ & $\begin{array}{c}c \\
( \pm 0.2 \mathrm{~km} / \mathrm{s})\end{array}$ & $\begin{array}{c}\phi \\
\left( \pm 2.5^{\circ}\right)\end{array}$ & $\begin{array}{l}\phi_{e} \\
\left(.^{\circ}\right)\end{array}$ \\
\hline 01 & 8.53 & 53.7 & 50.3 \\
\hline 02 & 8.57 & 55.2 & 53.2 \\
\hline 05 & 8.80 & 56.1 & \\
\hline 07 & 8.59 & 58.7 & 52.7 \\
\hline 08 & 8.72 & 57.9 & 54.2 \\
\hline 09 & 8.72 & 56.2 & 56.1 \\
\hline 15 & 8.72 & 60.8 & 57.0 \\
\hline 16 & 8.50 & 53.9 & 54.3 \\
\hline 17 & 8.45 & 50.8 & 52.1 \\
\hline 20 & 8.74 & 56.8 & 52.9 \\
\hline 26 & 8.76 & 56.6 & 55.5 \\
\hline 29 & 8.72 & 58.4 & 56.0 \\
\hline 30 & 8.55 & 51.5 & 52.5 \\
\hline $\begin{array}{c}\text { Average } \\
\text { (standard deviation) } \\
\end{array}$ & $\begin{array}{c}8.64 \\
(0.11) \\
\end{array}$ & $\begin{array}{l}55.7 \\
(2.9) \\
\end{array}$ & \\
\hline $\begin{array}{c}\text { Global Solution } \\
\text { (picks from } 21 \text { events) }\end{array}$ & 8.72 & 56.1 & \\
\hline \multirow[t]{7}{*}{$\begin{array}{l}\text { Station Residuals in seconds } \\
\text { (standard deviation in brackets) }\end{array}$} & & & \\
\hline & PDD & $-0.006(0.008)$ & \\
\hline & SAL & $0.012(0.012)$ & \\
\hline & RKK & $0.003(0.010)$ & \\
\hline & KSE & $-0.008(0.008)$ & \\
\hline & SEF & $0.000(0.012)$ & \\
\hline & NDE & $0.001(0.013)$ & \\
\hline
\end{tabular}


Table2. 4b Inversion results from the Weber data set, using the extended network

\begin{tabular}{|c|c|c|c|}
\hline $\begin{array}{l}\text { Event } \\
\text { I.D. }\end{array}$ & $\begin{array}{c}c \\
( \pm 0.2 \mathrm{~km} / \mathrm{s}) \\
\end{array}$ & $\begin{array}{c}\phi \\
\left( \pm 2.5^{\circ}\right) \\
\end{array}$ & $\begin{array}{l}\phi_{e} \\
\left(.^{o}\right)\end{array}$ \\
\hline 01 & 8.84 & 63.8 & 50.3 \\
\hline 02 & 8.81 & 61.8 & 53.2 \\
\hline 05 & 8.85 & 61.2 & \\
\hline 07 & 8.66 & 56.6 & 52.7 \\
\hline 09 & 8.95 & 52.4 & 56.1 \\
\hline 15 & 8.72 & 62.5 & 57.0 \\
\hline 16 & 8.93 & 59.3 & 54.3 \\
\hline 17 & 8.83 & 52.2 & 52.1 \\
\hline 18 & 8.96 & 61.4 & \\
\hline 20 & 8.76 & 61.5 & 52.9 \\
\hline 26 & 8.87 & 63.7 & 55.5 \\
\hline 29 & 8.72 & 64.9 & 56.0 \\
\hline 30 & 8.50 & 53.5 & 52.5 \\
\hline $\begin{array}{c}\text { Average } \\
\text { (standard deviation) } \\
\end{array}$ & $\begin{array}{c}8.80 \\
(0.13) \\
\end{array}$ & $\begin{array}{l}61.0 \\
(4.4) \\
\end{array}$ & \\
\hline \multicolumn{4}{|l|}{ Global Solution } \\
\hline & 8.80 & 58.8 & \\
\hline \multicolumn{4}{|l|}{$\begin{array}{l}\text { Station Residuals in seconds } \\
\text { (standard deviation in brackets) }\end{array}$} \\
\hline & PDD & $-0.008(0.036)$ & \\
\hline & SAL & $0.037(0.042)$ & \\
\hline & RKK & $0.017(0.035)$ & \\
\hline & KSE & $-0.011(0.030)$ & \\
\hline & SEF & $0.020(0.044)$ & \\
\hline & NDE & $0.016(0.031)$ & \\
\hline & WEL & $-0.045(0.032)$ & \\
\hline & CAW & $0.015(0.037)$ & \\
\hline & MRW & $-0.026(0.050)$ & \\
\hline
\end{tabular}


Table2. 4c Inversion results from other S. Hawkes Bay data, using the L-network

\begin{tabular}{|cccc|}
\hline $\begin{array}{c}\text { Event } \\
\text { I.D. }\end{array}$ & $\begin{array}{c}c \\
( \pm 0.2 \mathrm{~km} / \mathrm{s})\end{array}$ & $\begin{array}{c}\phi \\
\left( \pm 2.5^{\circ}\right)\end{array}$ & $\begin{array}{c}\phi_{e} \\
\left(.^{\circ}\right)\end{array}$ \\
\hline 102 & 8.43 & 58.0 & 55.9 \\
104 & 9.15 & 74.7 & 63.2 \\
107 & 8.21 & 54.0 & 55.7 \\
113 & 9.66 & 57.2 & 49.0 \\
115 & 9.04 & 63.0 & 56.9 \\
116 & 8.56 & 57.7 & 54.0 \\
119 & 8.69 & 61.4 & 55.1 \\
120 & 8.22 & 52.6 & 52.6 \\
121 & 8.24 & 53.2 & 52.6 \\
127 & 8.65 & 57.2 & 55.1 \\
129 & 8.44 & 49.6 & 51.5 \\
131 & 9.13 & 62.8 & 55.7 \\
\hline
\end{tabular}

Table2.4d Inversion results from the Lake Tennyson data set, using the L-network

\begin{tabular}{|c|c|c|c|}
\hline $\begin{array}{l}\text { Event } \\
\text { I.D. }\end{array}$ & $\begin{array}{c}c \\
( \pm 0.2 \mathrm{~km} / \mathrm{s})\end{array}$ & $\begin{array}{c}\phi \\
\left( \pm 2.5^{\circ}\right)\end{array}$ & $\begin{array}{l}\phi_{e} \\
\left({ }^{\circ}\right)\end{array}$ \\
\hline 302 & 9.68 & 220.4 & 236.0 \\
\hline 318 & 9.19 & 216.5 & 238.0 \\
\hline 328 & 10.26 & 223.0 & 237.8 \\
\hline $\begin{array}{c}\text { Average } \\
\text { (standard deviation) }\end{array}$ & $\begin{array}{c}9.71 \\
(0.53)\end{array}$ & $\begin{array}{r}220.0 \\
(3.27)\end{array}$ & 237.3 \\
\hline $\begin{array}{c}\text { Global Solution } \\
\text { (picks from } 5 \text { events) }\end{array}$ & 9.71 & 225.35 & \\
\hline $\begin{array}{l}\text { Station Residuals in seconds } \\
\text { (standard deviation in brackets) }\end{array}$ & $\begin{array}{l}\text { PDD } \\
\text { SAL } \\
\text { KSE } \\
\text { SEF } \\
\text { EGB } \\
\text { NDE }\end{array}$ & $\begin{array}{r}0.002(0.019) \\
-0.004(0.018) \\
-0.008(0.029) \\
0.000(0.009) \\
0.035(0.078) \\
-0.026(0.008)\end{array}$ & \\
\hline
\end{tabular}

The wavefront parameters $c$ and $\delta \phi$ appear to have no direct relation with the depths of the 
events, suggesting that the first-arriving energy is always the one that follows the implied highvelocity medium.

The apparent velocities given by the extended network (Table $2.4 \mathrm{~b}$ ) are noticeably higher than those from the L-network alone, giving an average of $8.80 \mathrm{~km} / \mathrm{s}$. There is however a much larger measure of unreliability associated with these inversions, due to the possible regional variation between the stations, which makes the assumption of a completely uniform wavefront less valid.

In summary, considering all available results and their reliability, we may take a working average of $8.7 \pm 0.2 \mathrm{~km} / \mathrm{s}$ and $6.0 \pm 2.5^{\circ}$ to represent the apparent velocity and azimuthal anomaly respectively for events in the Weber data set recorded at Wellington.

\subsubsection{Other events}

The higher quality events in the Central North Island which were chosen for inversion also yielded the high velocities given by the Weber data set, as well as the eastward-shifted azimuth of approach. The results are given in Table $2.4 \mathrm{c}$. In this case, no average value was calculated, and a global inversion was not carried out, since the events are scattered over too wide an area. Also, no reliable measure of the mislocation is available.

The South Island events (Table 2.4c), as expected, gave much higher apparent velocities since the wave paths from these sources were now updip. Also, the azimuthal anomalies are now of opposite sign to those form the North Island, and this is in agreement with the idea of the first arriving wave following a path within the dipping slab.

If we treat the Weber events and the Lake Tennyson events as lying on opposite ends of a reversed profile, with the Weber events yielding downdip paths and the Lake Tennyson events yielding updip paths, we can then estimate an approximate P-wave velocity for the refracting horizon from

$$
v=\frac{1}{2}\left(v_{\text {updip }}+v_{\text {downdip }}\right)
$$

Using the average values in Tables $2.4 \mathrm{a}$ and $2.4 \mathrm{~d}$, we get an average value of $9.2 \mathrm{~km} / \mathrm{s}$ ! This value however is only a first approximation, since as shall be seen in the next chapter, the actual geometry and morphology of the subducted slab along the wavepath, is an important factor in determining the wavefront at a particular point above the slab. 


\subsection{Travel Time/Distance Graph}

For the North Island events shown in Figure 2.9, the travel time of the first arrival to station $\mathrm{KSE}$ was calculated, and a graph of travel time against distance plotted. This graph is shown in Figure 2.13. The points were fitted to a best straight line having the equation

$$
t=\frac{1}{8.8 \pm 0.3} r+(5.2 \pm 0.2)
$$

indicating an apparent velocity of $8.8 \mathrm{~km} / \mathrm{s}$ along the average path sampled by refracted waves from these events. This is in quite good agreement with the values obtained in Table 2.4a-c.

The events for which this data was plotted were mostly within the subducted slab (see Appendix A). The three points in Figure 2.13 that lie substantially above the best-fit line in fact

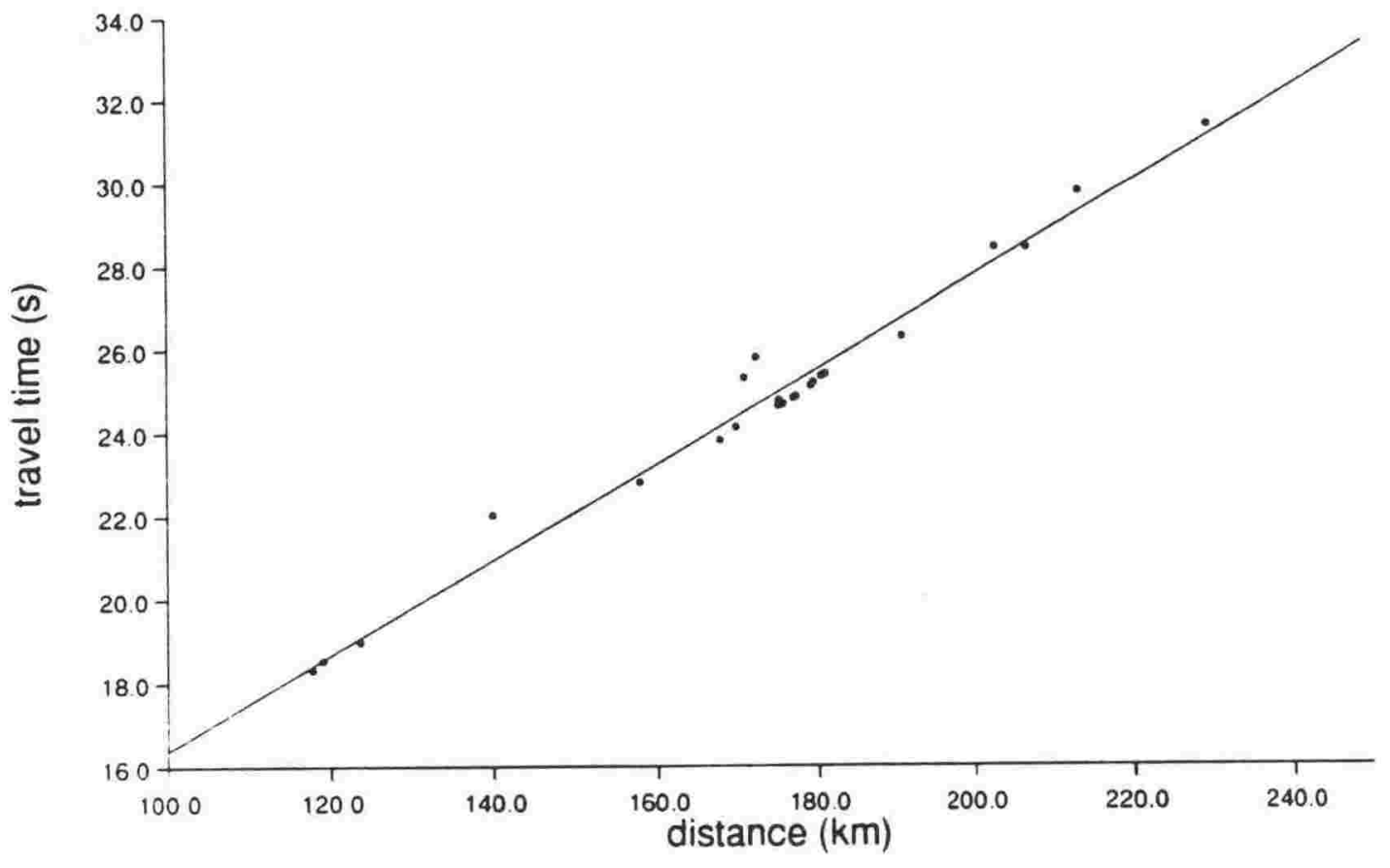

Fig. 2.13 First arrival travel time curve for North Island events recorded on $K S E$ 
correspond to three of the shallowest events in this data set, having depths less than $30 \mathrm{~km}$ (events 113,117 and 126 at distances $140.05 \mathrm{~km}, 171.34 \mathrm{~km}$ and $172.84 \mathrm{~km}$ respectively). Given that the first arrival is a refracted phase along some horizon within the slab, a time delay is expected to be introduced for these shallow sources.

\subsection{Particle Motions}

The off-azimuth character of the first arrivals implied by the results in Tables $2.4 \mathrm{a}-\mathrm{d}$ is supported by examining particle motion diagrams for the first phase of the seismograms. For each event, the N-S and E-W axes were rotated to radial (r) and transverse ( $t$ ) axes, where the positive radial axis was along the direction from epicentre to station. The projection of the particle motions on the $z-r, z-t$ and $r-t$ planes (where $z$ represents the vertical axis) for some selected
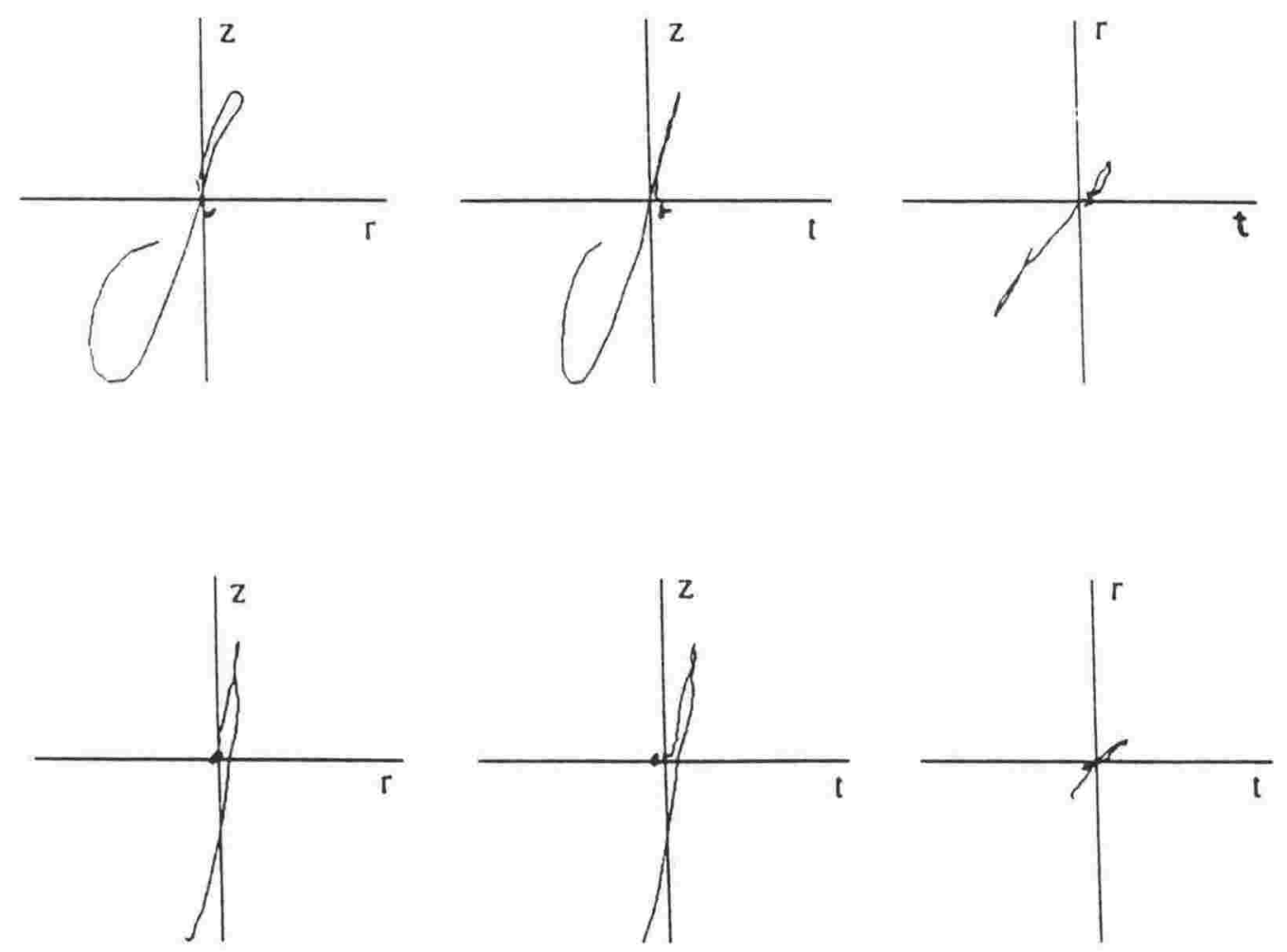

Fig. 2. 14 Particle motion diagrams for two selected events 
seismograms are shown in Figure 2.14. The time windows were 1s long and included some preevent noise. Since the first arrival was usually of small amplitude, the diagrams could only be plotted when the pre-event noise was low.

Looking at the r-t projections, the first direction of motion for many of the seismograms appears to be from a more easterly direction relative to the radial axis. Where this behaviour is observed, the r-t angle varies between about $10^{\circ}$ and $40^{\circ}$. Of the seismograms in which the preevent noise was low enough for the first motion to be well discerned, about $65 \%$ showed this polarization anomaly. This behaviour was more apparent in stations PDD, RKK, KSE and NDE than in the other two stations, SAL and SEF. The sections were sited on hilly terrain, many of them on hillside slopes. Buchbinder and Haddon (1990) have shown that the surface topography has a pronounced effect on the polarization vector of the P-wave. For this reason, although there appears to be a consistent eastward shift of the incident direction of the P-wave which supports the inversion results, it is difficult to make a quantitative evaluation of the polarization anomaly. No corrections for the topography effect have been attempted here. 


\section{Chapter 3}

\section{The Effects of Slab Geometry on the Speed and Azimuth of a Refracted Wavefront}

\subsection{Introduction}

The apparent velocities recorded at Wellington as discussed in Chapter 2 are much higher than average upper mantle P-velocities recorded worldwide, which are normally closer to 8.0 $8.2 \mathrm{~km} / \mathrm{s}$ (Suyehiro, 1988). High apparent velocities may either reflect intrinsically high Pvelocities in the medium, or else be produced as a result of the geometry of the refracting horizon, specifically by an updipping interface. The gross features of the geometry of the subducted slab below the North Island are such that the path from the Weber region to Wellington is downdip, which would tend to produce lower, rather than higher, apparent velocities at Wellington, implying that the intrinsic P-velocities are even higher than the apparent velocities recorded. There is still the possibility, however, that local departures from the assumed geometry may significantly affect the apparent velocity. Thus, it is unreasonable to reach any conclusion about the real P-velocicies below the North Island before taking the combined effect of intrinsic velocities and slab geometry fully into account.

In this chapter, some forward modelling is carried out, in which, for given velocity structures, the effects of slab shape and curvature on the observed apparent velocity are explored. In this modelling, the apparent wavefront speed as well as the wavefront azimuth of approach are predicted by a given structure and compared with the observations of these two parameters. The use of two such parameters provides a better constraint on the structure. The variations of the slab geometry are done within the bounds of previous observations, by other means, about the structure of the subducted slab. The effects on the apparent velocity of a refracted wavefront travelling within the slab in any direction are examined. Both a plane dipping slab, as well as a curved one, are explored by different techniques.

The case of a refracted wave travelling purely updip or downdip along a dipping refracting horizon, such as a dipping Moho, is well known, and the respective increase or decrease in apparent velocity as a function of the horizon dip and critical angle can be easily calculated. In the case of a wave travelling either along strike of a dipping refractor, or partially down- or updip, the situation is a little more complex, since the wavefront arriving at the receiver has an azimuth of approach that is shifted from the direct line between the source and receiver. It is then necessary to predict both the apparent speed and the azimuth of the wavefront, each of which is a 
function of the slab dip, the seismic velocities above and below the refracting horizon, as well as the take-off angle of the wave within the slab. This is the case with most source-receiver geometries for earthquakes in New Zealand, and in particular the Weber earthquakes recorded at Wellington. For these earthquakes, the source-receiver line makes an angle of about $10^{\circ}$ with the strike of the subducted plate in this region, and therefore the wavepath is partially downdip. For the $\mathrm{L}$. Tennyson earthquakes recorded at Wellington, the azimuth of the first arrival confirms that it also follows a path along the dipping slab, in this case the path being partially updip.

If the refracting horizon is not planar but curved, then the azimuthal shift still occurs but is not as easy to describe analytically. Such a situation is probably present beneath the North Island, although in some regions, the slab may be approximated as planar.

The problem of interpretation of apparent velocities and azimuths in terms of dipping structures has been tackled at arrays in California (Otsuka, 1966 (a), (b)) and Arizona (Niazi, 1966). These two studies, however, dealt only with plane interfaces.

\subsection{A Plane Dipping Refractor}

\subsubsection{The Downdip Case}

Since our main data set to be modelled is that of the Weber earthquake sequence, we shall consider first the case of a partially downdip travel path, which is illustrated in Figure 3.1. The plane boundary $\mathrm{ABCD}$, dipping at angle $\delta$, separates two homogeneous media having $\mathrm{P}$-wave velocities $v_{1}$ and $v_{2}$. The critical angle is therefore

$$
\gamma=\sin ^{-1}\left(\frac{v_{1}}{v_{2}}\right)
$$

For simplicity the ray path is assumed to originate at $\mathrm{O}$, on the refracting horizon, at depth $h$ below the surface, and to travel down the slab, making an angle $\alpha$ with the strike, within the slab. It then refracts off the slab at $\mathbf{R}$.

The Fermat path of least time for a ray travelling from $O$ to a receiver $L$ at the surface must lie in a plane containing $\mathrm{L}$ and the normal $\mathrm{ON}$ to the dipping surface at $\mathrm{O}$. Using this principle, we can first find an expression for the angle $\alpha$, which is constrained by the dip $\delta$ and the relative positions of $\mathrm{O}$ and $\mathrm{L}$. The derivation is given in Appendix $\mathrm{C}$, and the expression is given by:

$$
\tan \alpha=\left(\frac{X}{Y}\right) \cos \delta
$$

$\mathrm{X}$ and $\mathrm{Y}$ are the coordinates of $\mathrm{L}$ relative to an origin at $\mathrm{N}$, the point where the normal to the slab at $\mathrm{O}$ intersects the surface.

The wavefront apparent velocity as measured at a small network at $\mathrm{L}$ is found by evaluating 


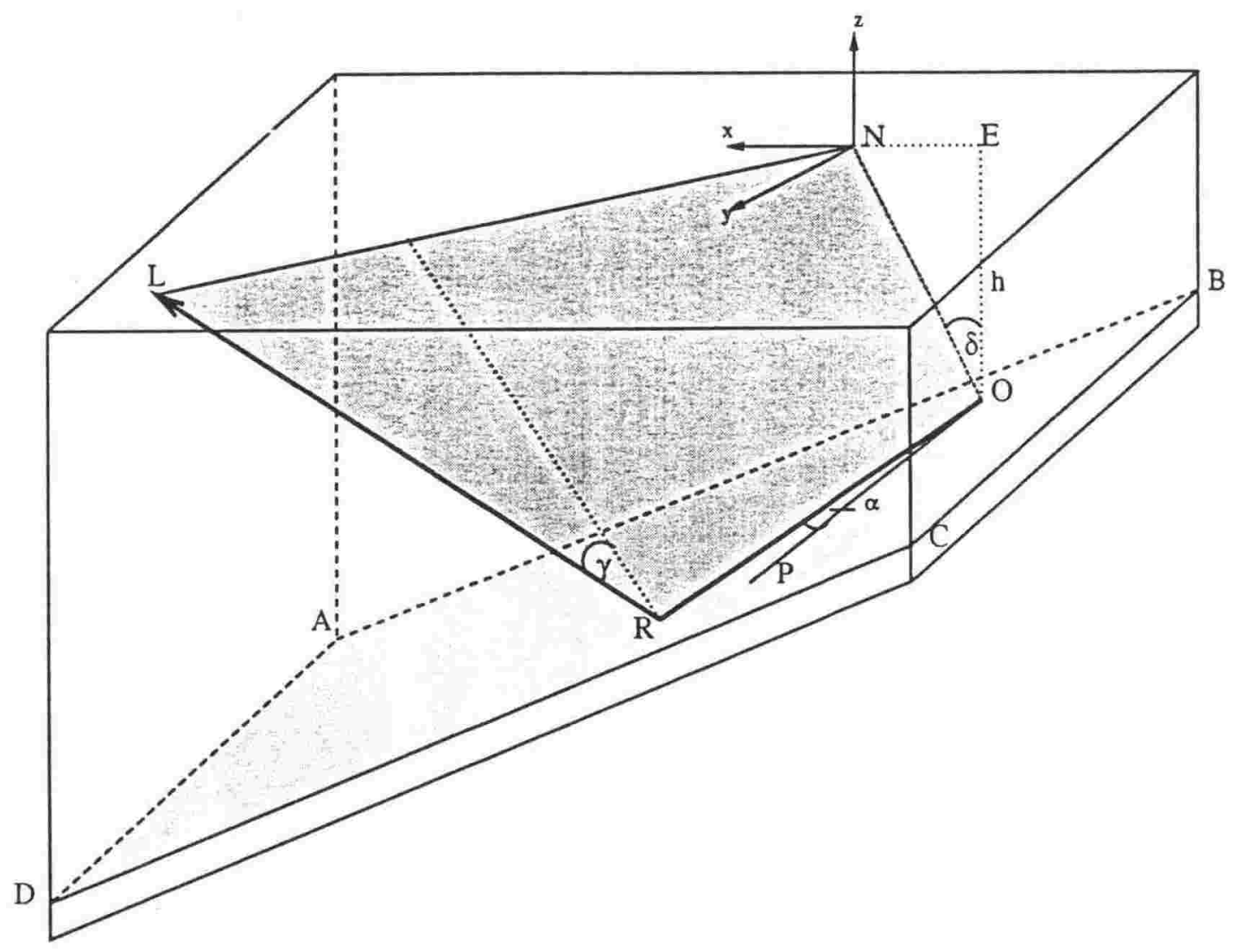

Fig. 3.1 Geometrical setting for a refracted ray travelling along a plane dipping interface. The velocities in the upper and lower media are $v_{1}$ and $v_{2}$ respectively.

the horizontal slowness vector $\mathrm{p}$ at $\mathrm{L}$. Now for a ray travelling across any number of parallel boundaries, such as in a horizontally layered medium, Snell's law states that the horizontal slowness is constant. However, at a point such as $\mathrm{R}$, where the boundary is not horizontal, there is a change in the slowness $\mathbf{p}$. We can evaluate this change in $\mathrm{p}$ by making a simple change of coordinate systems at $\mathrm{R}$. Consider Figure 3.2, in which $x, y, z$ is the frame of reference representing the vertical and horizontal axes, and $x^{\prime}, y^{\prime}, z^{\prime}$ are the coordinate axes defining the dipping slab. Thus $z^{\prime}$ lies along the normal to the dipping slab at $\mathrm{R}, x^{\prime}$ lies along the dip and $y^{\prime}$ lies along strike, coinciding with axis $y$.

From the laws of refraction, the refracted ray at $\mathrm{R}$ lies in the same plane as the $z^{\prime}$ axis and the ray travelling in the slab. $\gamma$ is the critical angle made by the refracted ray with the $z^{\prime}$ axis. A unit vector along the direction of the refracted ray has coordinates with respect to the $x^{\prime}, y^{\prime}, z^{\prime}$ axes given by 


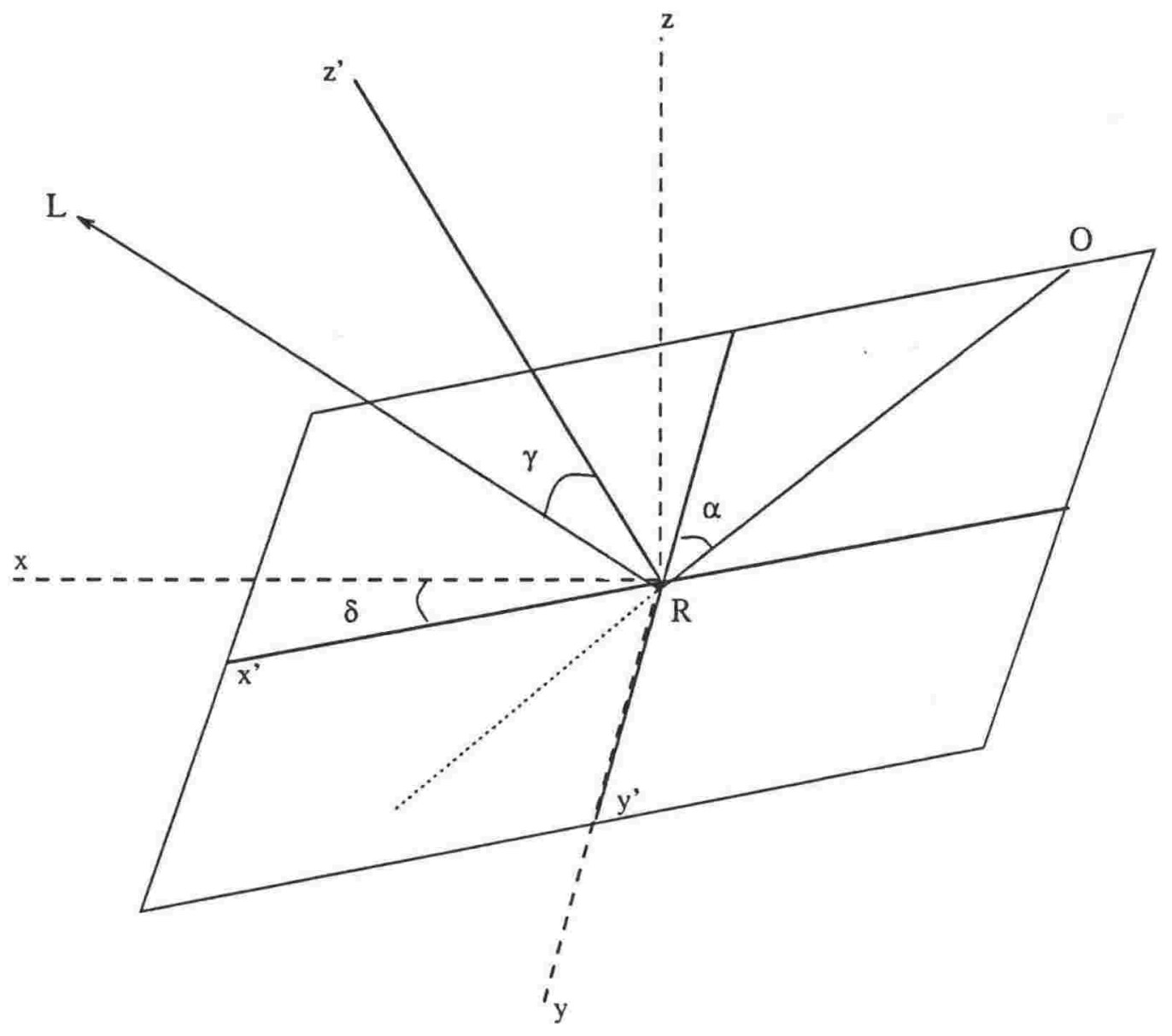

Fig. 3.2 Change of coordinate system at the point where the ray refracts off the dipping interface. $\delta$ is the dip of the interface, $\gamma$ is the critical angle, $\alpha$ is the take-off angle of the ray within the slab.

$$
(\sin \gamma \sin \alpha, \sin \gamma \cos \alpha, \cos \gamma)^{T}
$$

We simply have to write this vector in terms of the $x, y, z$ frame of reference. This is achieved by multiplying the vector by the rotation matrix

$$
\left(\begin{array}{ccc}
\cos \delta & 0 & \sin \delta \\
0 & 1 & 0 \\
-\sin \delta & 0 & \cos \delta
\end{array}\right)
$$

Thus the final slowness vector relative to the $x, y, z$ axes is given by

$$
\mathbf{s}=s\left(\begin{array}{ccc}
\cos \delta & 0 & \sin \delta \\
0 & 1 & 0 \\
-\sin \delta & 0 & \cos \delta
\end{array}\right)\left(\begin{array}{c}
\sin \gamma \sin \alpha \\
\sin \gamma \cos \alpha \\
\cos \gamma
\end{array}\right)
$$




$$
\mathbf{s}=\frac{1}{v_{1}}\left(\begin{array}{c}
\cos \delta \sin \gamma \sin \alpha+\sin \delta \cos \gamma \\
\sin \gamma \cos \alpha \\
-\sin \delta \sin \gamma \sin \alpha+\cos \delta \cos \gamma
\end{array}\right)
$$

This is also referred to as the polarization vector.

The horizontal components of s yield the horizontal slowness vector $\mathrm{p}$ observed at the surface. Rewriting these components in terms of $v_{2}$, we get

$$
\mathbf{p}(\alpha)=\frac{1}{v_{2}}(\cos \delta \sin \alpha+\cot \gamma \sin \delta, \cos \alpha)
$$

Note that as a result of Snell's law, no amount of horizontal layering in the upper medium will change the value of $\mathbf{p}$ once the ray has left $\mathrm{R}$, which means that the velocity $v_{1}$ in effect refers to the velocity immediately above the dipping boundary. (Of course, changes in velocity as the ray travels up to the surface from $R$, will dictate the point where the ray hits the surface, and thus will slightly influence the slowness $\mathbf{p}$ in the sense that $\alpha$ must vary accordingly).

From equation 3.2 , we may write expressions for the magnitude and direction of the slowness vector at any point $(X, Y)$. Thus

$$
\begin{gathered}
c=\frac{1}{\sqrt{p_{x}^{2}+p_{y}^{2}}} \\
c=v_{2}\left(\cos ^{2} \alpha+\cot ^{2} \gamma \sin ^{2} \delta+\sin 2 \delta \sin \alpha \cot \gamma+\cos ^{2} \delta \sin ^{2} \alpha\right)^{-\frac{1}{2}} \\
\tan \phi_{s}=\left(\frac{p_{x}}{p_{y}}\right) \\
\tan \phi_{s}=\cos \delta \tan \alpha+\frac{\cot \gamma \sin \delta}{\cos \alpha}
\end{gathered}
$$

where $\phi_{s}$ is the azimuth relative to the strike of the slab (or y-axis).

The azimuthal anomaly $\delta \phi$ is then given by the difference between the angle $\phi_{s}$ made by $\mathrm{p}$ with the $y$-axis, and the angle $\phi_{e s}$ made by the source-receiver line with the $y$-axis:

$$
\begin{gathered}
\tan \phi_{e s}=\frac{X+h \tan \delta}{Y} \\
\delta \phi=\phi_{s}-\phi_{e s}
\end{gathered}
$$

$\delta \phi$ is the angle that would be measured between the radial and horizontal components of the horizontal projection of the particle motion vector at the surface (Figure 3.3).

For source-receiver geometries such that the angle $\alpha$ is small, and for epicentral distances 


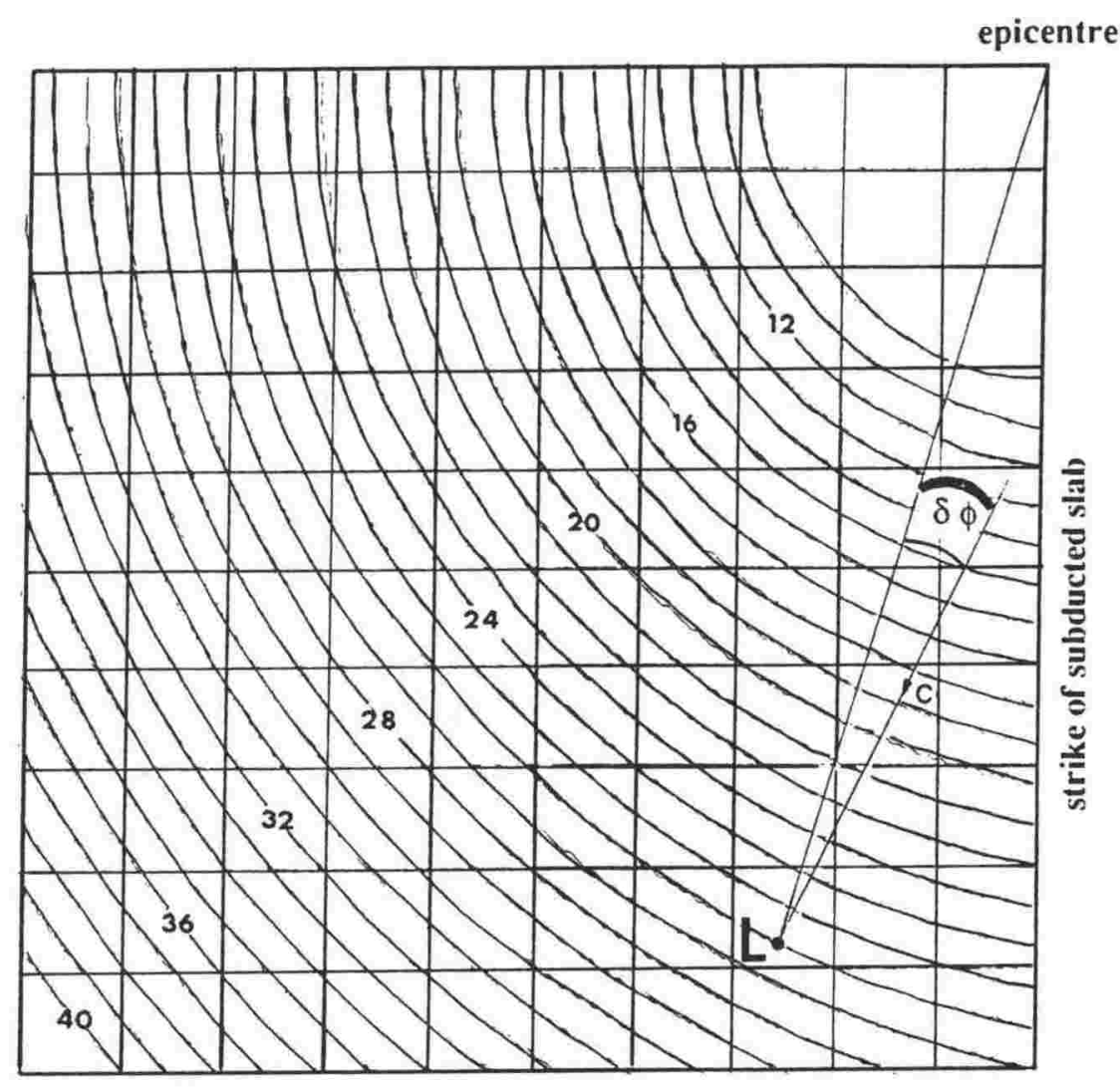

$\stackrel{20 \mathrm{~km}}{\longrightarrow}$

Fig. 3.3 Equal travel-time contours defining the wavefront observed at the earth's surface due to the wave path geometry of Figure 8. $L$ lies on the 26 s contour, and the contour spacing is 1.0s. These contours have been generated for the case when $v_{1}=6.3 \mathrm{~km} / \mathrm{s}, v_{2}=8.6 \mathrm{~km} / \mathrm{s}$, $\delta=10^{\circ}$ and $h=32 \mathrm{~km}$. The wavefront observed at $L$ is along the vector c. $\delta \phi$ is the azimuthal anomaly.

such that $\mathrm{Y} \gg \mathrm{X}$, the expressions for $c$ and $\delta \phi$ may be replaced by the approximations

$$
c=v_{2}\left(1+\cot ^{2} \gamma \sin ^{2} \delta+\sin ^{2} \delta \cot \gamma \sin \alpha\right)^{-\frac{1}{2}}
$$

and

$$
\tan (\delta \phi)=\cot \gamma \sin \delta
$$

Note that while the wavefront speed $c$ is directly dependent on both the velocity $v_{2}$ and the ratio of velocities $v_{1}$ and $v_{2}$ (through the factor $\cot \gamma$ ), the azimuthal anomaly $\delta \phi$ depends only on their ratio.

Refraction along a dipping boundary means that the horizontal projection of the wavefront 
along the earth's surface is not circular, as in the case of a horizontal refracting horizon, but elliptical. Figure 3.3 illustrates the contours of equal travel-time due to a source-receiver geometry as in Figure 3.1, in the case when $\delta=10^{\circ}, h=34 \mathrm{~km}, v_{1}=6.3 \mathrm{~km} / \mathrm{s}$, and $v_{2}=8.6$ $\mathrm{km} / \mathrm{s}$. The direction of the wavefront at $\mathrm{L}$ is represented by the normal at $\mathrm{L}$, and demonstrates the shift of the azimuth of the refracted arrival relative to the epicentre-receiver line. Appendix C contains the derivation of the travel-time of a ray such as ORL, and the equations of the elliptical wavefronts in Figure 3.3. It also demonstrates how, from the travel-time, the slowness vector may be derived in an alternative way.

An interesting and convenient result follows from equation 3.2 by noting that the component of the slowness along strike, $p_{y}$, is given by

$$
p_{y}=\frac{1}{c} \cos \phi_{s}
$$

and therefore

$$
\frac{1}{c} \cos \phi_{s}=\frac{1}{v_{2}} \cos \alpha
$$

Thus

$$
\frac{c}{\cos \phi_{s}}=v_{2}\left(1+\tan ^{2} \alpha\right)^{\frac{1}{2}}
$$

or

$$
\frac{c}{\cos \phi_{s}}=v_{2}\left(1+\frac{X^{2}}{Y^{2}} \cos ^{2} \delta\right)^{\frac{1}{2}}
$$

Thus it follows that if the slab and source-receiver geometries are known or assumed, then the observed values of $c$ and $\phi_{s}$ between them constrain the refractor velocity $v_{2}$ that must be present, independently of the upper medium velocity $v_{1}$.

Figure 3.4 illustrates graphically the effect of slab dip on the wavefront apparent velocity. $c$ and $\delta \phi$ have been computed as a function of the angle made by the source-receiver line with the strike direction at an epicentral distance of $175 \mathrm{~km}$. The variations are shown for slab dips of $5^{\circ}$, $10^{\circ}$ and $15^{\circ}$, with the slab surface at $34 \mathrm{~km}$ depth at the source in all cases. The upper and lower medium P-wave velocities have been arbitrarily taken as $6.3 \mathrm{~km} / \mathrm{s}$ and $8.6 \mathrm{~km} / \mathrm{s}$ respectively. The effect of an increase in the slab dip on both $c$ and $\delta \phi$ is quite strong. Note that there is no azimuthal anomaly at $90^{\circ}$, i.e. in the pure downdip case.

If we make the assumption of a plane dipping slab in the region between Southern Hawkes Bay and Wellington, we can use equation 3.4 and our average observed values of $c$ and $\phi_{s}$ from the Weber data set to make an estimate of the velocity $v_{2}$ below the refracting horizon. Some assumptions have to be made here. First of all, $\phi_{s}$ requires the strike of the subducted slab in the 

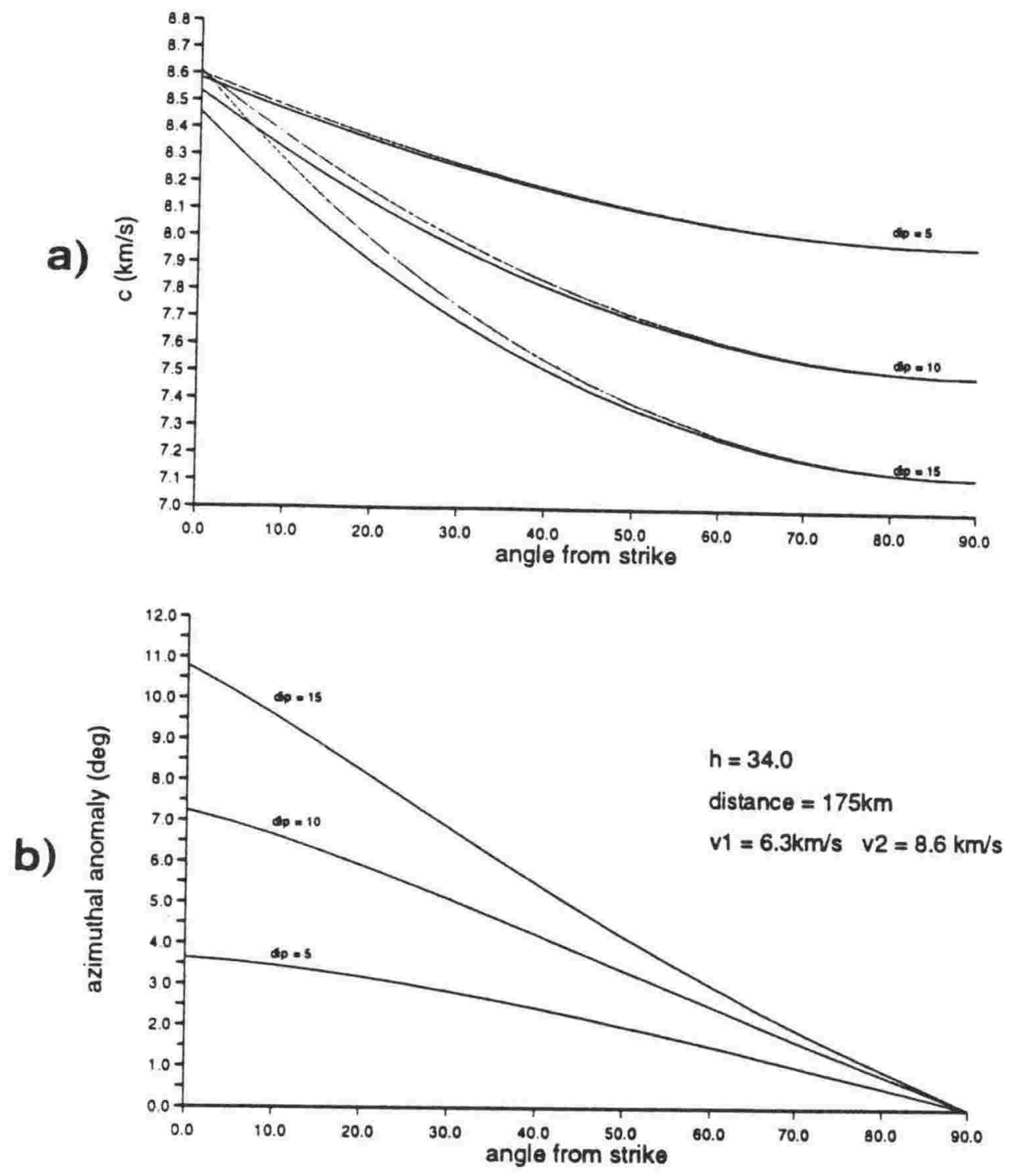

Fig. 3.4 Effect of slab dip on wavefront characteristics, as a function of epicentre-station azimuth downdip from the strike direction. An angle of $0^{\circ}$ represents a source-receiver line exactly along strike. (a) Variation of wavefront speed, $c$ (solid line) and apparent speed $\frac{c}{\cos \delta \phi}$, measured along a line joining source to receiver (dotted line); (b) Azimuthal anomaly.

lower North Island to be known. Estimates of this have ranged between $\mathrm{N} 45^{\circ} \mathrm{E}$ (Reyners, 1989) and $\mathrm{N} 40^{\circ} \mathrm{E}$ (Ansell and Bannister, 1991). We shall concur with the most recent data and use a 
strike of $\mathrm{N} 40^{\circ} \mathrm{E}$. Using the average azimuth of $56.0^{\circ}$ calculated by the array travel time inversions in Section 2.5, an average value of $16.0^{\circ}$ is obtained for $\phi_{s}$.

Secondly, an average position $X, Y$ of the receiver relative to the source must be chosen. With the "average epicentre" used in Section 2.5.1, and a source depth of $30 \mathrm{~km}, X, Y$ were calculated to be $34 \mathrm{~km}$ and $170 \mathrm{~km}$ respectively.

Based on the above assumptions, Table 3.1 shows the velocity $v_{2}$ that would be required for different values of dip $\delta$ in the case of a plane slab. A value for $v_{1}$ can then also be calculated using the value of $\cot \gamma$ given by 3.3b. It is seen that for given observed values of $c$ and $\phi_{s}$, the required value of $v_{2}$ is approximately fixed over quite a large range of the slab dip, but that the required values of $v_{1}$ undergo a much larger variation, between 4.59 and $7.72 \mathrm{~km} / \mathrm{s}$.

\subsubsection{The Updip Case}

In the case of a ray travelling partially updip, as in the L. Tennyson events recorded at Wellington. it is straightforward to show that the vector $\mathbf{s}$ becomes

$$
s=\frac{1}{v_{1}}\left(\begin{array}{c}
-\cos \delta \sin \gamma \sin \alpha+\sin \delta \cos \gamma \\
-\sin \gamma \cos \delta \\
\sin \delta \sin \gamma \sin \alpha+\cos \delta \cos \gamma
\end{array}\right)
$$

thus

$$
\mathbf{p}=\frac{1}{v_{2}}(-\cos \delta \sin \alpha+\cot \gamma \sin \delta, \cos \alpha)
$$

Table3. 1 For a plane slab geometry, the velocities

$v_{1}$ and $v_{2}$ which would produce observed values of $8.70 \mathrm{~km} / \mathrm{s}$ and $16.0^{\circ}$ for $c$ and $\phi_{s}$ respectively, at varying slab dip

\begin{tabular}{|ccccc|}
\hline $\begin{array}{c}\delta \\
\text { (degrees) }\end{array}$ & $\begin{array}{c}v_{2} \\
(\mathrm{~km} / \mathrm{s})\end{array}$ & $\begin{array}{c}\alpha \\
\text { (degrees) }\end{array}$ & cot $\gamma$ & $\begin{array}{c}v_{1} \\
(\mathrm{~km} / \mathrm{s})\end{array}$ \\
\hline 4 & 8.92 & 9.30 & 1.67 & 4.59 \\
6 & 8.93 & 8.81 & 1.20 & 5.72 \\
8 & 8.94 & 8.54 & 0.94 & 6.51 \\
10 & 8.95 & 8.15 & 0.80 & 6.99 \\
12 & 8.96 & 7.74 & 0.71 & 7.32 \\
14 & 8.96 & 7.32 & 0.64 & 7.45 \\
16 & 8.97 & 6.90 & 0.59 & 7.72 \\
\hline
\end{tabular}


giving

$$
\begin{gathered}
c=v_{2}\left(\cos ^{2} \alpha+\cot ^{2} \sin ^{2} \delta-\sin 2 \delta \sin \alpha \cot \gamma+\cos ^{2} \delta \sin ^{2} \alpha\right)^{-\frac{1}{2}} \\
\tan \phi_{s}=-\cos \delta \tan \alpha+\frac{\cot \gamma \sin \delta}{\cos \alpha}
\end{gathered}
$$

In this case, the expression for $\alpha$ would still be as equation 3.1, however the value of $X$ is now $X_{O}+h \tan \delta$. Likewise, the equation 3.4 will have the same form, but the value of $X$, together with the fact that $\phi_{s}$ will now be smaller than $\phi_{e s}$ (as opposed to the downdip case) will mean that $c$ will be larger than $v_{2}$.

\subsection{A Cylindrically Curved Refractor}

Separate microearthquake studies conducted in the Hawkes Bay and Wellington regions (e.g. Reyners, 1979; Bannister, 1988; Robinson, 1986) confirm that the dip of the subducting slab along the east coast is smaller than that below Wellington, increasing from about $5^{\circ}$ to about $15^{\circ}$. More detailed studies of the results from these and a number of other surveys in the lower North Island by Ansell and Bannister (1991) have revealed that this change in dip is in fact consistent with the morphology of the slab being cylindrical in this region. More exactly, the surface of the slab can be closely approximated by a cylindrical curvature which varies along the strike of the subducted slab. Such a shape has been described by the authors as most resembling a "rolled ball" and agrees with the mathematically expected curvature for a bending plate on a spherical surface. For the case of the North Island, New Zealand, the "cylinder" axis strikes $\mathrm{N} 40^{\circ} \mathrm{E}$ and lies just off the east coast. The radius of curvature at the top of the subducted plate varies from about $200 \mathrm{~km}$ in northern Hawkes Bay to about $300 \mathrm{~km}$ at Wellington. Further inland, beneath the Taupo Volcanic Centre, the dip increases abruptly and the plate becomes much more planar, so that this kind of curvature is believed to be confined to the initial stages of bending of the slab.

In this section we shall neglect the change in the radius of curvature, and represent the slab surface between Hawkes Bay and Wellington by a simple cylinder of radius, $\rho, 270 \mathrm{~km}$, whose axis lies $286 \mathrm{~km}$ below the free surface. Following Ansell and Bannister (1991), the perpendicular distance from Wellington to the line at the surface directly above the cylinder axis (physically, where the subducted plate begins to bend down) is taken as $70 \mathrm{~km}$. Interfaces below the subducted slab surface would then correspond to smaller radii of curvature.

The main consideration to be made in the case of a cylindrically shaped slab as opposed to a plane dipping one is the exact point at which the ray refracts off the slab, and hence the dip at that 
point, since it is this dip which determines the final vector. Figure 3.5 illustrates the situation, again in the simplified case where the ray originates at the interface between the two media. For situations in which the station-epicentre line makes a small angle with the strike direction, the ray travels very close to the interface, and the geometry may be taken as identical to that in Figure 3.2 , where the dipping plane now represents the tangent plane at point $R$. The same expression 3.2 for wavefront slowness will apply, in which the value of $\delta$ is to be replaced by the local slab $\operatorname{dip} \delta_{R}$, where, for a cylinder of radius $\rho$.

$$
\delta_{R}=\sin ^{-1}\left(\frac{x_{R}}{\rho}\right)
$$

and $x_{R}$ is the distance of $\mathrm{R}$ from the $\mathrm{y}$-axis in Figure 3.4.

The path taken by the ray, and hence the value of $\delta_{R}$, depends on the velocities $v_{1}$ and $v_{2}$. For a given source and target position, the refraction point $\mathbf{R}$ was not found analytically, but by

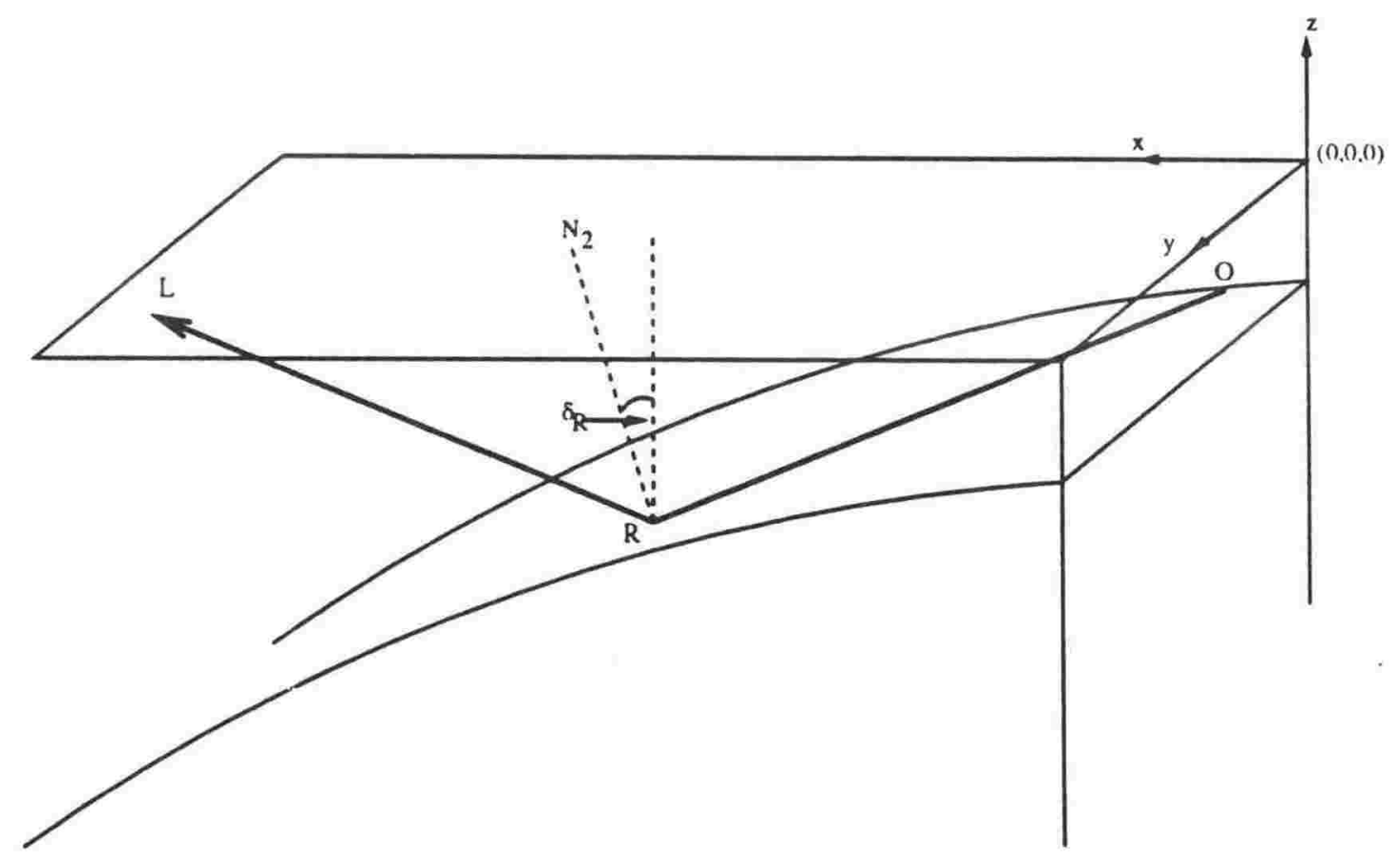

Fig. 3.5 A cylindrically curved slab. P-wave velocities above and below the interface are $v_{1}$ and $v_{2}$ respectively. 
using a simple ray-tracing procedure and searching for the coordinates of $\mathrm{R}$ by trial and error. When the coordinates of $\mathrm{R}$ were found, it was possible to calculate $\delta_{R}$, and then the problem could be treated in the same manner as a plane dipping slab of equivalent dip $\delta_{R}$.

Table 3.2 shows some results of this procedure, and indicates that, for various combinations of parameters, and for the source-receiver geometry of the Weber earthquakes relative to Wellington, the dip at the point of refraction does not vary much from an average of about $11^{\circ}$. Using $11^{\circ}$ as the slab dip in expression 3.4 yields a value of $8.95 \mathrm{~km} / \mathrm{s}$ for $v_{2}$.

The last two columns of Table 3.2 show the expected values of $c$ and $\delta \phi$ for the wavefront at Wellington and indicate the extent to which both $c$ and $\delta \phi$ can be individually fitted. It is hard to reconcile both the high speeds and the observed azimuthal anomaly without very high values of

Table3.2. Wavefront characteristics at $L$ due to refraction off a cylindrical surface

\begin{tabular}{|ccc|ccc|ccc|}
\hline $\begin{array}{c}v_{1} \\
(\mathrm{~km} / \mathrm{s})\end{array}$ & $\begin{array}{c}v_{2} \\
(\mathrm{~km} / \mathrm{s})\end{array}$ & $\begin{array}{c}h \\
(\mathrm{~km})\end{array}$ & $\begin{array}{c}x_{R} \\
(\mathrm{~km})\end{array}$ & $\begin{array}{c}y_{R} \\
(\mathrm{~km})\end{array}$ & $\begin{array}{c}\delta_{R} \\
(\text { degrees })\end{array}$ & $\begin{array}{c}c \\
(\mathrm{~km} / \mathrm{s})\end{array}$ & $\begin{array}{c}\phi_{s} \\
(\text { degrees })\end{array}$ & $\begin{array}{c}\delta \phi \\
(\text { degrees })\end{array}$ \\
\hline 6.5 & 8.6 & 32 & 16 & 124 & 12.3 & 8.27 & 17.4 & 5.0 \\
6.5 & 8.8 & 32 & 16 & 127 & 12.3 & 8.45 & 17.8 & 4.6 \\
6.5 & 9.0 & 32 & 16 & 129 & 12.3 & 8.62 & 18.3 & 4.1 \\
6.5 & 9.2 & 32 & 17 & 131 & 12.5 & 8.77 & 19.1 & 3.3 \\
& & & & & & & & \\
7.0 & 8.6 & 32 & 15 & 115 & 12.1 & 8.35 & 15.6 & 6.8 \\
7.0 & 8.8 & 32 & 15 & 118 & 12.1 & 8.53 & 16.0 & 6.4 \\
7.0 & 9.0 & 32 & 16 & 120 & 12.2 & 8.69 & 16.7 & 5.7 \\
7.0 & 9.2 & 32 & 16 & 124 & 12.3 & 8.86 & 17.3 & 5.1 \\
& & & & & & & & \\
7.5 & 8.6 & 32 & 13 & 99 & 11.6 & 8.43 & 13.8 & 8.6 \\
7.5 & 8.8 & 32 & 14 & 106 & 11.8 & 8.60 & 14.2 & 8.2 \\
7.5 & 9.0 & 32 & 14 & 110 & 11.8 & 8.77 & 14.8 & 7.6 \\
7.5 & 9.2 & 32 & 15 & 114 & 12.1 & 8.94 & 15.7 & 6.7 \\
& & & & & & & & \\
8.0 & 8.6 & 32 & 10 & 70 & 10.9 & 8.50 & 11.9 & 10.5 \\
8.0 & 8.8 & 32 & 11 & 84 & 11.2 & 8.67 & 12.4 & 10.0 \\
8.0 & 9.0 & 32 & 12 & 94 & 11.4 & 8.84 & 12.9 & 9.5 \\
8.0 & 9.2 & 32 & 13 & 101 & 11.6 & 9.01 & 13.7 & 8.7 \\
& & & & & & & & \\
\hline
\end{tabular}


$v_{2}$. The effect of $v_{1}$ on the wavefront can also be seen from the table. The higher the velocity $v_{1}$, the lower the velocity $v_{2}$ required to produce a given apparent velocity, however the azimuthal anomaly produced becomes smaller. It should be remembered here that the velocity $v_{1}$ refers to the medium immediately above the interface, and therefore in the real situation of a layered upper medium, $v_{1}$ need only be confined to a layer above the subducted slab surface.

\subsection{3-Dimensional Ray Tracing}

The results of the previous sections indicate that the observed wavefront speed and azimuth require P-wave velocities of at least $8.9 \mathrm{~km} / \mathrm{s}$ below the refracting horizon in the subducted slab. Such velocities are well in excess of P-wave velocities normally encountered in oceanic or continental upper mantle. It was therefore suspected that the high apparent velocities might be, at least partly, caused by some geometrical effect related to the shape of the subducted slab. As discussed in Section 3.3, the work of Ansell and Bannister has revealed that the morphology of the subducted slab is not of perfectly cylindrical shape, but is characterized by a varying radius of curvature on going along strike. It was therefore decided to investigate also the extent to which such a departure from the previously assumed cylindrical curvature would affect the apparent velocity.

For this purpose, use was made of a complete 3-dimensional ray tracing (CRT) program (Cerveny, Klimes and Psencik, 1988). In this program, an arbitrary, 3-dimensional structure of any complexity may be modelled, with the velocity defined at as many grid nodes as required, thus allowing any amount of lateral or vertical velocity gradient, besides first-order discontinuities. An appropriate ray code enables the user to trace a ray from any source position through any desired sequence of reflections and/or transmissions at the interfaces. The final slowness vector of the ray at the surface enables the apparent velocity and azimuth of the wavefront to be calculated.

In this application, the ray tracing was carried out by gradually adjusting the two take-off angles (in the horizontal and vertical planes) of the ray from the source point until the target point was reached. As the program had no graphic or automatic target finding capabilities at the time of use, this was a highly time-consuming procedure, because the nature of the curvature of the interfaces meant that small changes in one or both of the take-off angles could produce relatively large deviations of the final ray vector. The final adjustments were carried out in steps of less than 0.0001 radians.

With the use of this program it was also possible to model more realistic structures and 
situations, in particular placing the source at different depths, having a layered medium both above and below the subducted slab surface, and also tracing different ray types, such as reflections. The program was first used to test the approximate predictions of the simple cylindrical model of the previous section.

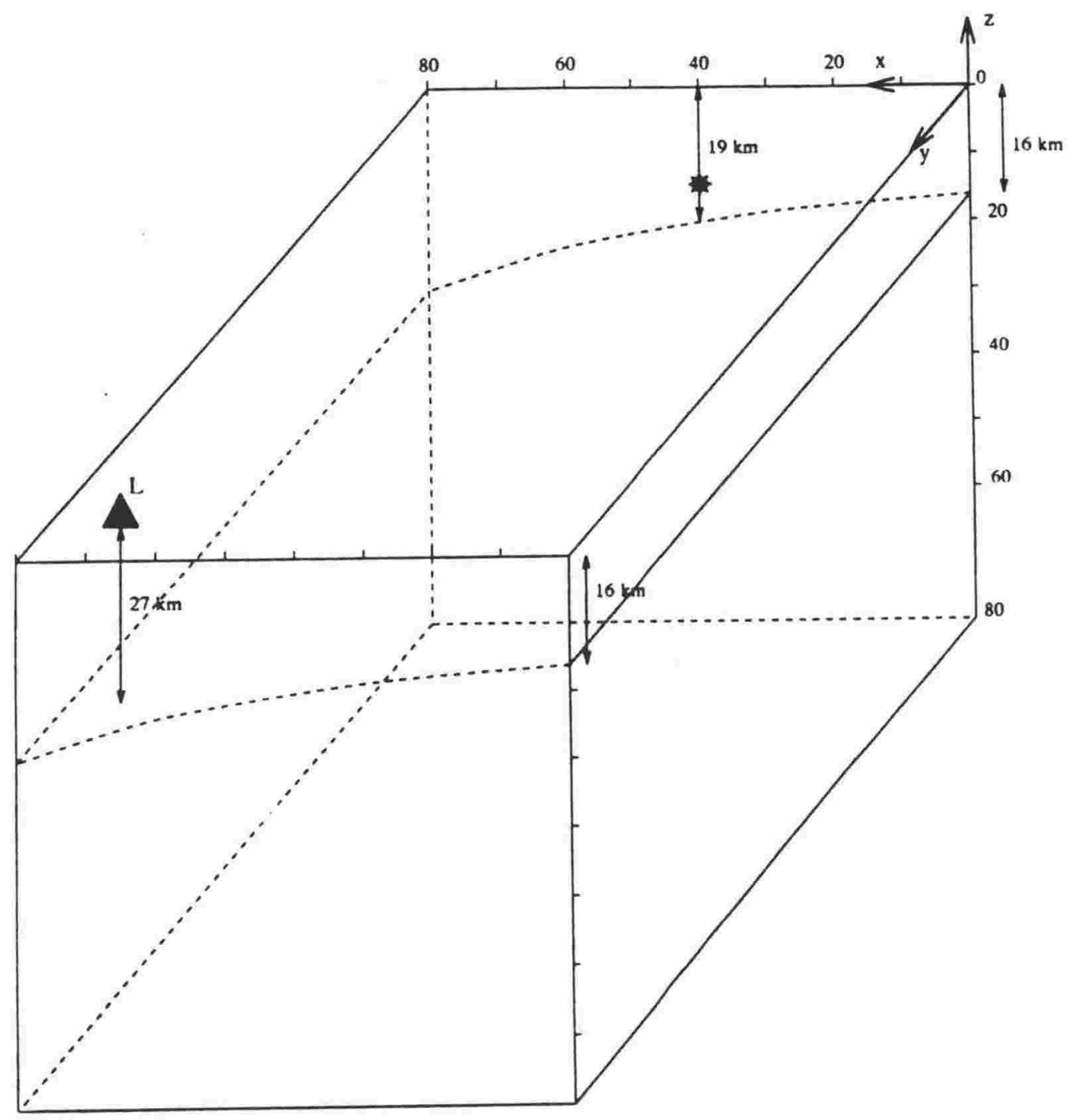

Fig. 3.6 Cylindrical curvature model constructed for 3-d ray tracing. The star represents the source position. $L$ represents the station position. 


\subsubsection{Regular Cylindrical Curvature}

The basic model constructed had the form and dimensions shown in Figure 3.6. The position of the source is shown by a star and was based upon the "average epicentre" of $40.3 \mathrm{~S}$, 176.3E used in Chapter 2. It was placed at $40 \mathrm{~km}$ from the $\mathrm{y}$-axis. Its depth was varied between 15 and $30 \mathrm{~km}$. The position of the target point, based on the coordinates of station KSE, was placed at $x=70 \mathrm{~km}$ and $y=170 \mathrm{~km}$, giving an epicentral distance of $173 \mathrm{~km}$. The slab surface was kept fixed, with its depth below the free surface calculated at different points according to the cylinder geometry used in the previous section. This resulted in a slab surface depth of $19 \mathrm{~km}$ at the source position and $25.2 \mathrm{~km}$ at the receiver (target) position. Additional interfaces were added both above the curved surface, as well as below, during the modelling. "Head waves" were generated by using slight gradients within the layers, although in most cases, the curvature of the surface was enough to ensure that a ray travelled just below an interface and emerged at some other point, without the need for a velocity gradient.

As a check on the predictions of the previous section for a cylindrically shaped slab surface, some models were constructed which had velocities corresponding to some of those in Table 3.2. Intermediate layers were added, but the velocities $v_{1}$ and $v_{2}$ in Table 3.2 always corresponded to the velocity immediately above the slab surface and that through which the ray was "critically" refracted respectively. These were the only two velocities which had a significant effect on the final slowness vector at $L$.

Figure 3.7 shows some cross-sections of the models used, together with the resulting slowness vector at $\mathrm{L}$ and the travel time, in the left-hand side of the accompanying table. All of these results refer to the ray critically refracted through the $8.8 \mathrm{~km} / \mathrm{s}$ medium. These models are not intended to provide conclusions about the velocity structure but merely to illustrate the effects of the various parameters. Models (c) and (d) are designed to show that the depth of the source has a very small effect on the apparent velocity at $\mathrm{L}$. Likewise the effect of the depth of the refracting horizon is shown by comparing (b) and (c). Again the effect of this parameter is slight. On the other hand, the effect of velocity $v_{1}$ just above the slab surface is quite pronounced, as seen in (a) and (b).

All in all, there is little difference in the case of a regular cylindrical surface between the results of the 3-dimensional ray tracing and the simple geometrical techniques used in the previous section, and it is evident that the only parameters which significantly affect the slowness vector are the curvature of the surface and the two velocities $v_{1}$ and $v_{2}$, as would be expected. 


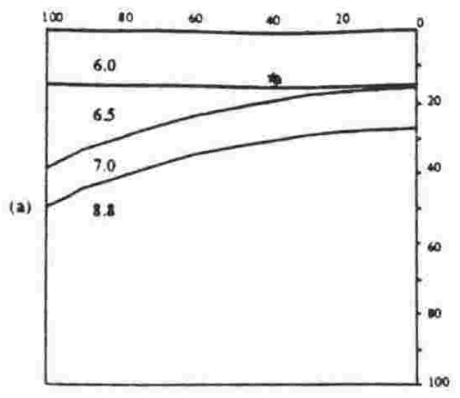

Cylindrical Curvature Modified Curvature

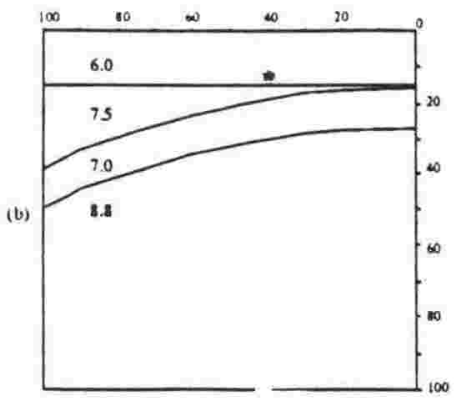

$$
\begin{aligned}
& t=24.5 \mathrm{~s} \\
& \mathrm{c}=8.41 \mathrm{~km} / \mathrm{s} \\
& \phi_{\mathrm{s}}=19.6^{\circ}
\end{aligned}
$$

$$
\begin{aligned}
& \mathrm{t}=24.1 \mathrm{~s} \\
& \mathrm{c}=8.59 \mathrm{~km} / \mathrm{s} \\
& \phi_{\mathrm{s}}=20.6^{\circ}
\end{aligned}
$$

$$
\begin{aligned}
& t=23.8 \mathrm{~s} \\
& \mathrm{c}=8.60 \mathrm{~km} / \mathrm{s} \\
& \phi_{\mathrm{s}}=16.4^{\circ}
\end{aligned}
$$$$
\mathrm{t}=23.9 \mathrm{~s}
$$$$
\mathrm{c}=8.71 \mathrm{~km} / \mathrm{s}
$$$$
\phi_{s}=16.7
$$

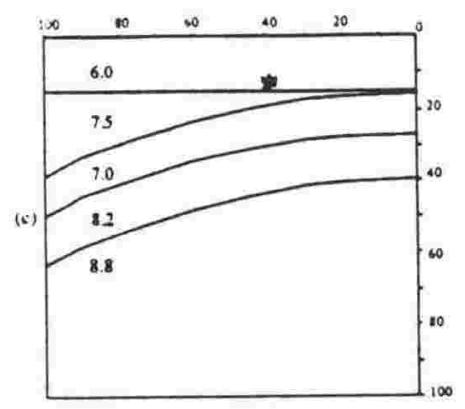

$$
\begin{aligned}
& t=25.6 \mathrm{~s} \\
& c=8.62 \mathrm{~km} / \mathrm{s} \\
& \phi_{\mathrm{s}}=15.0^{\circ}
\end{aligned}
$$

$$
\begin{aligned}
& t=25.6 \mathrm{~s} \\
& c=8.73 \mathrm{~km} / \mathrm{s} \\
& \phi_{\mathrm{s}}=16.2^{\circ}
\end{aligned}
$$

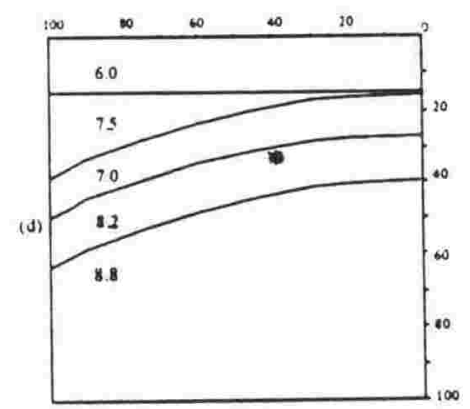

$$
\begin{aligned}
& t=24.4 \mathrm{~s} \\
& c=8.60 \mathrm{~km} / \mathrm{s} \\
& \phi_{\mathrm{s}}=15.5
\end{aligned}
$$

Fig.3.7 Cross-sections of the 3-d models used to demonstrate the effect of slab shape and velocity structure on travel time and wavefront velocity. In the case of the modified curvature, the cross-section is at the Hawkes Bay latitude. The star represents the source position

\subsubsection{Effect of Non-cylindrical Curvature}

The main reason for using 3-d ray tracing was to test the effect on the wavefront of departures of the slab surface from a simple cylindrical shape, since this would be difficult to reproduce analytically. Figure 3.8 shows the new model constructed, following the proposals of 
Ansell and Bannister (1991), while Table 3.3 gives more details of the model construction.

As can be seen in the figure, there is a slight updip component along any line parallel to the strike of the subducted slab. In this model, the uppermost slab surface below point $A$ at the $S$. Hawkes Bay latitude is $5 \mathrm{~km}$ deeper than that below point B at the Wellington latitude, so that the slab surface along this line has a dip of about $1.6^{\circ}$. The slab surface at the Wellington latitude was calculated as a cylinder of radius $290 \mathrm{~km}$, while that at the S.Hawkes Bay latitude had a radius of curvature of $235 \mathrm{~km}$. The curvatures at some intermediate latitudes were taken proportionally, and the program then constructed a smooth continuous surface. This model places the slab surface at approximate depths of $19 \mathrm{~km}$ at the source position, and $20 \mathrm{~km}$ below the receiver at Wellington - a smaller difference in depths than that which results from a regular cylindrical shape. The $20 \mathrm{~km}$ depth at Wellington is slightly less than that in Robinson's (1986) model $(22 \mathrm{~km})$ but this will not significantly affect the ray path, which is closer to the shallower

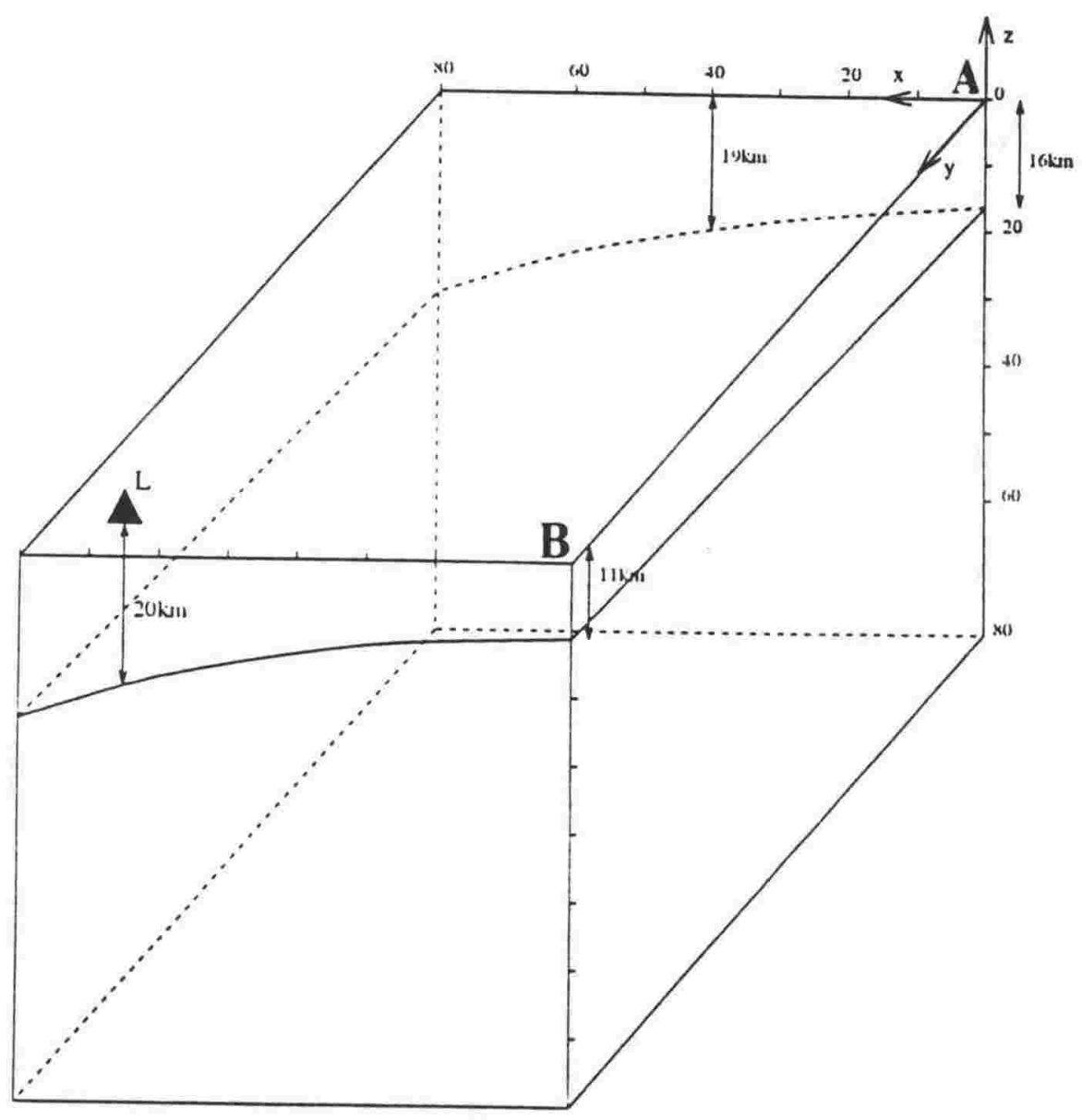

Fig. 3.8 Modified cylindrical curvature model constructed for 3-d ray tracing following Ansell and Bannister (1991). The star represents the source position. $L$ represents the station position 
end of the slab.

Some effects of the geometry can be seen in the right-hand column of the table in Figure 3.7. In this figure, the same velocities have been kept as the ones in the cylindrical case, so that the effect of slab shape can be compared. These effects are quite significant. Due to decreased curvature on going from S. Hawkes Bay to Wellington, the apparent velocity at Wellington for any given refractor velocity $v_{2}$ is higher than would be observed for a simple cylindrical surface of constant curvature. Indeed for a receiver situated directly along strike, as in the Hikurangi

Table3.3 Construction of 3-d model used in CRT with modified cylindrical geometry. Values in tables represent depths below the surface for the subduction interface and the two interfaces below. $x$ and $y$ values are in $\mathrm{km}$, and relative to the origin defined in Figure 3.8.

1st curved interface

\begin{tabular}{c|cccc}
\hline $\mathrm{x}$ & 0 & 30 & 60 & 90 \\
\hline 0 & -16.0 & -17.9 & -23.7 & -33.7 \\
\hline 85 & -13.5 & -15.2 & -20.4 & -29.4 \\
\hline 170 & -11.0 & -12.6 & -17.3 & -25.4 \\
\hline
\end{tabular}

2nd curved interface

\begin{tabular}{c|cccc}
$\mathrm{y}^{\mathrm{x}}$ & 0 & 30 & 60 & 90 \\
\hline 0 & -27.0 & -28.9 & -34.7 & -44.7 \\
\hline 85 & -24.5 & -26.2 & -31.4 & -40.4 \\
\hline 170 & -22.0 & -23.6 & -28.3 & -36.4 \\
\hline
\end{tabular}

3rd curved interface (variable)

\begin{tabular}{c|cccc}
$\mathrm{y}^{\mathrm{x}}$ & 0 & 30 & 60 & 90 \\
\hline 0 & -40.0 & -41.9 & -47.7 & -57.7 \\
\hline 85 & -37.5 & -39.2 & -44.4 & -53.4 \\
\hline 170 & -35.0 & -36.6 & -41.3 & -49.4 \\
\hline
\end{tabular}


Margin refraction experiment, one would expect to measure an increased apparent velocity relative to $v_{2}$, although there would still be a small off-azimuth component due to the sideways dip.

Because the depth of the refracting interface has such a small effect on the slowness, then modelling of the apparent velocity and azimuth alone cannot be used to cunstrain the depth of the high velocity medium. Nor can it tell us anything about other velocities present. There are, however, two other constraints that can be made - the absolute travel time of the ray, and the relative travel times of the rays critically refracted at the various interfaces, keeping in mind that the refracted ray through the high-velocity medium must be the first arrival at Wellington. The use of 3-d ray tracing turns out to be a powerful tool in such a situation because it would be too unreliable to use approximate travel times from either the simpler models of the previous sections or from a 2-d ray tracing program.

In Chapter 2, it was seen that, for those aftershocks accurately located by the microearthquake network, a "shallow" event at $15 \mathrm{~km}$ depth results in a travel time to Wellington of about 26.5s. The average velocities of the model in Figure 3.7a, for example, coupled with the shallow depth ( $12 \mathrm{~km}$ beneath the slab surface) of the high velocity medium, gives a travel time of $23.82 \mathrm{~s}$, which is too short compared with the observed time for shallow sources. Longer travel times were most effectively, and reasonably, produced by pushing the high velocity medium deeper, and introducing a "normal" $8.0-8.2 \mathrm{~km} / \mathrm{s}$ upper mantle velocity, as in Figures $3.7 \mathrm{c}$ and d. An upper limit to the depth at which the high velocity medium can exist is imposed by the point at which the head wave through the $8.0-8.2 \mathrm{~km} / \mathrm{s}$ medium overtakes the head wave through the higher velocity medium. This is a useful constraint because it does not depend at all on the velocities above the two media, but only on the separation between them. Further modelling shows that, for the $8.7-8.8 \mathrm{~km} / \mathrm{s}$ head wave to arrive first at Wellington, the high-velocity medium cannot be deeper than about $30 \mathrm{~km}$ beneath the surface of the subducted slab. It is of course not possible to make any deductions about the thickness of this high-velocity medium.

Figure 3.9 shows one model that gives a reasonably good fit of wavefront speed, wavefront azimuth, as well as travel time. The curvature of the slab used was the modified one of Figure 3.8. The average velocity in the overlying crust is similar to that of Robinson (1986) for the Wellington region. The azimuth of the wavefront obtained with this model is a little higher than the average observed one of $16.0^{\circ}$ but still within the scatter of the data.

Although there is still a trade-off between the velocities $v_{1}$ and $v_{2}$, the observed values of wavefront speed, azimuth and travel time still provide adequate constraints on some velocities that must be present below the lower half of the North Island. The use of 3-d ray tracing has also made it much easier to understand the extent to which the slab curvature affects the apparent velocity.

Whereas the plane dipping slab approximation or the simple cylindrical geometry require a P-velocity of at least $8.9 \mathrm{~km} / \mathrm{s}$ within the subducted slab to explain the observations, if we take 


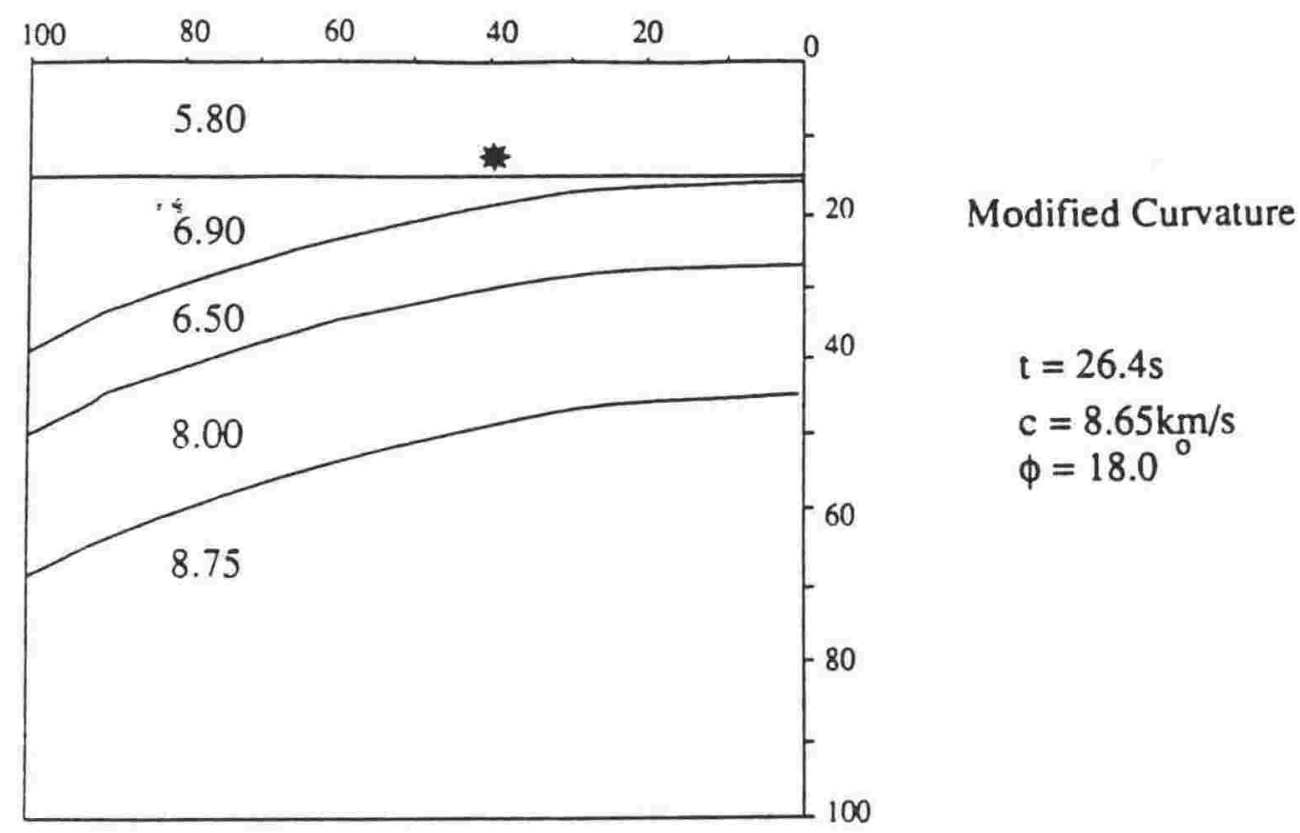

Fig. 3.9 One velocity model that gives a good fit of both wavefront speed and azimuth.

into account the modified slab geometry, we can get a good explanation with an $8.75 \pm 0.1 \mathrm{~km} / \mathrm{s}$ $\mathrm{P}$-velocity. This is still an unusually high upper mantle P-velocity. The effect of geometry could be taken further by considering more severe distortions in the subducted slab, such as sudden "warps" in the slab surface, or local rises in the topography of the slab surface. These could no doubt be designed to produce high apparent velocities if they were contrived to occur at the point where the ray leaves the surface of the slab. However, it must be kept in mind that high apparent velocities in this part of the North Island have been previously observed, and for travel path geometries different from the ones considered here, so that it would be difficult to use local irregularities in the slab surface morphology to explain every occurrence of high apparent velocity. Moreover, the seismicity studies of Ansell and Bannister (1991) do not suggest any such features other than the change of curvature.

In summary then, it is reasonable to conclude that the high apparent velocities measured in this study $(8.7 \mathrm{~km} / \mathrm{s})$, combined with the off-azimuth nature of the wavefront, are consistent with a slightly higher P-velocity of $8.75 \pm 0.1 \mathrm{~km} / \mathrm{s}$ at a depth of not more than $30 \mathrm{~km}$ below the surface of the subducted slab. 


\section{Chapter 4}

\section{The Reflectivity Method}

\subsection{Introduction}

This chapter will describe the basic theory underlying the "reflectivity method", which is an encompassing term for various techniques that calculate the complete response of a plane stratified medium to excitation by a source. The source is usually an explosive source or a double-couple approximation to an earthquake source. There are several such techniques, all of which involve the two basic procedures (a) computation of the complex response of a stack of uniform layers to a point source outside or within the stack, this response being at a particular frequency and slowness, and (b) some form of double integration over frequency and slowness (or wavenumber) to give the complete surface displacement in the time domain, at the desired spatial location relative to the source. The best known algorithms and procedures are those of Fuchs and Muller (1971), Kind (1976, 1978, 1979) and Kennett (1983). A good overview of the method is also given in Muller (1985).

For the purpose of modelling the general waveform features in this study, it was decided to use the approach of Kennett (1983) because of its greater numerical stability, and greater flexibility in simulating specific parts of the seismic wavefield. This made the identification of phases on the synthetic seismograms easier. The basic program source code was taken from Kennett (1988) and the computations were run on the UNIX-based Silicon Graphics mainframe computer of the Computer Services Centre, V.U.W.

\subsection{Matrix Representation of Plane Waves}

In a homogeneous medium, the propagation of a plane wave may be described by the motion-stress vector, $\mathbf{f}=\mathbf{f}(\mathbf{z})$ in that medium, where $z$ represents depth below the free surface. For $\mathrm{SH}$-waves, this vector is 2-component:

$$
\mathbf{f}_{S H}=\left(\begin{array}{l}
u_{y} \\
\tau_{y z}
\end{array}\right)
$$


where

$u_{y}$ is the displacement in the $y$-direction, in a reference frame in which propagation takes place along $\mathrm{x}$;

$\tau_{y z}$ is the component of the traction acting in the z-direction across the plane $y=0$.

For P-SV waves, the motion stress vector is 4-component:

$$
\mathbf{f}_{P S V}=\left(\begin{array}{l}
u_{x} \\
u_{z} \\
\tau_{z x} \\
\tau_{z z}
\end{array}\right)
$$

The components of $\mathbf{u}$ and $\tau$ are all assumed to have a time variation of the form $e^{i \omega(p x-t)}$.

For any type of plane wave, the equations of motion may be written in the form

$$
\frac{\partial \mathbf{f}}{\partial z}=\mathbf{A f}
$$

(Aki and Richards, 1980, pl63) where $\mathbf{A}$ is some matrix of constants, whose elements depend on the elastic constants of the medium, the angular frequency $\omega$ of the wave, and the horizontal slowness, or ray parameter $p$.

In the case of $\mathrm{SH}$ waves, the matrix $\mathbf{A}$ is a simple (2x2) matrix:

$$
\mathbf{A}_{S H}=\left(\begin{array}{cc}
0 & \frac{1}{\mu} \\
\left.\omega^{2(} \mu p^{2}-\rho\right) & 0
\end{array}\right)
$$

where $\mu$ is the rigidity modulus and $\rho$ is the density.

It is straightforward to show that with this matrix, equation (4.1) is equivalent to the equations of motion and Hooke's law:

$$
\begin{gathered}
\rho \ddot{v}=\frac{\partial \tau_{y z}}{\partial z}+\frac{\partial \tau_{y x}}{\partial x} \\
\tau_{y z}=\mu \frac{\partial v}{\partial z} ; \tau_{y x}=\mu \frac{\partial v}{\partial x}
\end{gathered}
$$

applicable to SH-waves. 
In the case of P-SV waves, the matrix $\mathrm{A}$ is $(4 \times 4)$ and somewhat more complex:

$$
\mathbf{A}_{P S V}=\left(\begin{array}{cccc}
0 & -i \omega p & \frac{1}{\mu} & 0 \\
\frac{-i \omega p \lambda}{\lambda+2 \mu} & 0 & 0 & \frac{1}{\lambda+2 \mu} \\
\frac{4 \omega^{2} p^{2} \mu(\lambda+\mu)}{\lambda+2 \mu}-\rho \omega^{2} & 0 & 0 & \frac{-i \omega p \lambda}{\lambda+2 \mu} \\
0 & -\rho \omega^{2} & -i \omega p & 0
\end{array}\right)
$$

By considering a function of the form

$$
f_{i}(z)=v_{i} e^{\lambda_{i}\left(z-z_{0}\right)}
$$

where $\lambda_{i}$ and $v_{i}$ are an eigenvalue and corresponding eigenvector of matrix $\mathbf{A}$ and $z_{o}$ is some reference level, it is easily shown that the general solution of (1) may be expressed as

$$
f(z)=F \cdot w
$$

where $\mathbf{F}$ is a $(2 \times 2)$ or $(4 \times 4)$ matrix consisting of the solutions $v_{i} e^{\lambda_{i}\left(z-z_{o)}\right)}$ as its columns, and $\mathbf{w}$ is a $(2 \times 1)$ or $(4 \times 1)$ matrix of constants which weight the different solutions. $F$ is called the layer, or fundamental, matrix for the particular medium and may be explicitly found in the two cases of SH and P-SV propagation by calculating the eigenvalues and eigenvectors of $\mathbf{A}_{S H}$ and $\mathbf{A}_{P S V}$.

For SH-waves:

$$
\mathbf{F}=\left(\begin{array}{cc}
e^{i \omega \eta\left(z-z_{o}\right)} & e^{-i \omega \eta\left(z-z_{o}\right)} \\
i \omega \rho \beta^{2} \eta e^{i \omega \eta\left(z-z_{o}\right)} & -i \omega \rho \beta^{2} \eta e^{-i \omega \eta\left(z-z_{o}\right)}
\end{array}\right)
$$


For P-SV waves:

$$
\mathbf{F}=\left(\begin{array}{cc}
\alpha p e^{i \omega \xi\left(z-z_{0}\right)} & \beta \eta e^{i \omega \eta\left(z-z_{0}\right)} \\
\alpha \xi e^{i \omega \xi\left(z-z_{0}\right)} & -\beta \rho e^{i \omega \eta\left(z-z_{o}\right)} \\
2 i \omega \rho \alpha \beta^{2} p \xi e^{i \omega \xi\left(z-z_{0}\right)} & i \omega \rho \beta\left(1-2 \beta^{2} p^{2}\right) e^{i \omega \eta\left(z-z_{o}\right)} \\
i \omega p \alpha\left(1-2 \beta^{2} p^{2}\right) e^{i \omega \xi\left(z-z_{0}\right)} & -2 i \omega \rho \beta^{3} p \eta e^{i \omega \eta\left(z-z_{o}\right)} \\
\alpha p e^{-i \omega \xi\left(z-z_{o}\right)} & \beta \eta e^{-i \omega \eta\left(z-z_{o}\right)} \\
-\alpha \xi e^{-i \omega \xi\left(z-z_{o}\right)} & \beta p e^{-i \omega \eta\left(z-z_{o}\right)} \\
-2 i \omega \rho \alpha \beta^{2} p \xi e^{-i \omega \xi\left(z-z_{o}\right)} & -i \omega \rho \beta\left(1-2 \beta^{2} p^{2}\right) e^{-i \omega \eta\left(z-z_{o}\right)} \\
i \omega \rho \alpha\left(1-2 \beta^{2} p^{2}\right) e^{-i \omega \xi\left(z-z_{0}\right)} & -2 i \omega \rho \beta^{3} p \eta e^{-i \omega \eta\left(z-z_{o}\right)}
\end{array}\right)
$$

where

$\alpha$ and $\beta$ are the compressional and shear wave velocities respectively, related to $\lambda$ and $\mu$ by

$$
\alpha^{2}=\frac{\lambda+2 \mu}{\rho} ; \beta^{2}=\frac{\mu}{\rho}
$$

$\xi$ and $\eta$ are the vertical slownesses of $\mathrm{P}$ and $\mathrm{S}$ waves respectively, given by

$$
\xi^{2}=\left(\frac{1}{\alpha^{2}}-p^{2}\right) ; \eta^{2}=\left(\frac{1}{\beta^{2}}-p^{2}\right)
$$

Note that the vertical phase factors $e^{ \pm i \omega \xi\left(z-z_{0}\right)}$ and $e^{ \pm i \omega \eta\left(z-z_{0}\right)}$ represent down- and upgoing $\mathrm{P}$ and $\mathrm{S}$ waves respectively. By the correct choice of the elements of the weighting matrix $w$ then, any combination of down- and upgoing waves can be represented by the formulation (5). For example, in the case of equation (4.5) applied to P-SV waves, the choice of $w=(1,0,0,0)^{T}$ yields the stress-displacement vector of a pure downgoing P-wave.

This representation of plane waves by a medium-dependent matrix enables the total wavefield at a given depth below the free surface to be expressed as the appropriate sum of contributions from upgoing and downgoing P-SV and SH waves. This is the basic formulation underlying most methods of calculating the response of a stratified medium to an incident plane wave. Note that evanescent waves are represented by (7) in the case when the value of $p$ is such that $\xi$ and $\eta$ become imaginary, i. e. $p>\alpha, p>\beta$. 


\subsection{The Stress-Displacement Propagator Matrix P}

The classical reflectivity method, as described, for example by Fuchs and Muller (1971), considers a stack of plane layers of homogeneous media whose upper surfaces lie at depths $z_{1}, z_{2}, \ldots z_{n}$ below the free surface $z=0$ respectively, and calculates the overall reflectivity of the stack using the idea of propagator matrices The propagator matrix is an operator which acts on the stress-displacement matrix at depth $z_{A}$ to give the stress-displacement matrix at depth $z_{B}$.

$$
\mathbf{f}\left(z_{B}\right)=\mathbf{P}\left(z_{B}, z_{A}\right) \mathbf{f}\left(z_{A}\right)
$$

If $z_{A}$ and $z_{B}$ lie within the same uniform layer, then $\mathbf{P}\left(z_{B}, z_{A}\right)$ is called the layer matrix for that layer, and simply transfers $f$ from the top to the bottom of the layer, or vice versa

It follows that

$$
\mathbf{P}\left(\mathbf{z}_{B}, z_{A}\right)=\mathbf{F}\left(z_{B}\right) \mathbf{F}^{-1}\left(z_{A}\right)
$$

so that the propagator matrices for each layer can be found. The explicit forms of $\mathbf{P}\left(z_{B}, z_{A}\right)$ for P-SV and SH waves, and the properties of propagator matrices are discussed in Aki and Richards (1980). In particular, propagator matrices obey the chain rule

$$
\mathbf{P}\left(z_{A}, z_{C}\right)=\mathbf{P}\left(z_{A}, z_{B}\right) \mathbf{P}\left(z_{B}, z_{C}\right)
$$

so that, for the stack of layers in Figure 4.1, the stress-displacement vectors at the upper and lower boundaries of the stack are related as follows

$$
\begin{gathered}
\mathbf{f}\left(z_{1)}=\mathbf{P}\left(z_{1}, z_{n}\right) \mathbf{f}\left(z_{n}\right)\right. \\
\mathbf{f}\left(z_{1)}=\mathbf{P}\left(z_{1}, z_{2}\right) \mathbf{P}\left(z_{2}, z_{3}\right) \cdots \mathbf{P}\left(z_{n-1}, z_{n}\right) \mathbf{f}\left(z_{n}\right)\right.
\end{gathered}
$$

given that there is no change in the stress-displacement vectors in going across interfaces. The propagator matrix for the whole stack can thus be found as the product of the individual layer matrices. As a brief illustration of the principles involved in this method, consider a downgoing $\mathrm{P}$ wave incident on the stack. In the upper half-space we have downgoing $\mathrm{P}$ and upgoing $\mathrm{P}$ and $\mathrm{S}$ waves, while in the lower half-space we assume that we have only downgoing $\mathrm{P}$ and $\mathrm{S}$ waves. If $F_{u}$ and $F_{l}$ are the fundamental matrices for the upper and lower half-spaces, we have

$$
\begin{aligned}
& \mathbf{f}\left(z_{1}\right)=\mathrm{F}_{u}\left(1,0 R_{P P}, R_{P S}\right)^{T} \\
& \mathbf{f}\left(z_{n}\right)=\mathrm{F}_{l}\left(T_{P P}, T_{P S}, 0,0\right)^{T}
\end{aligned}
$$

where $R_{P P}, R_{P S}, T_{P P}, T_{P S}$ are the reflection and transmission coefficients for the whole stack, and a power of $T$ denotes a vector transpose. The reference depths $z_{o}$ for the phase factors in $F_{u}$ and $\mathbf{F}_{l}$ may be arbitrarily taken as $z_{1}$ and $z_{n}$ respectively, so that the phase terms $e^{ \pm \omega \xi\left(z-z_{o}\right)}$ and $e^{ \pm i \omega \eta\left(z-z_{0}\right)}$ are all in turn equal to 1 .

Between equations (4.8) and (4.9) we have a system of 4 equations in 4 unknowns 


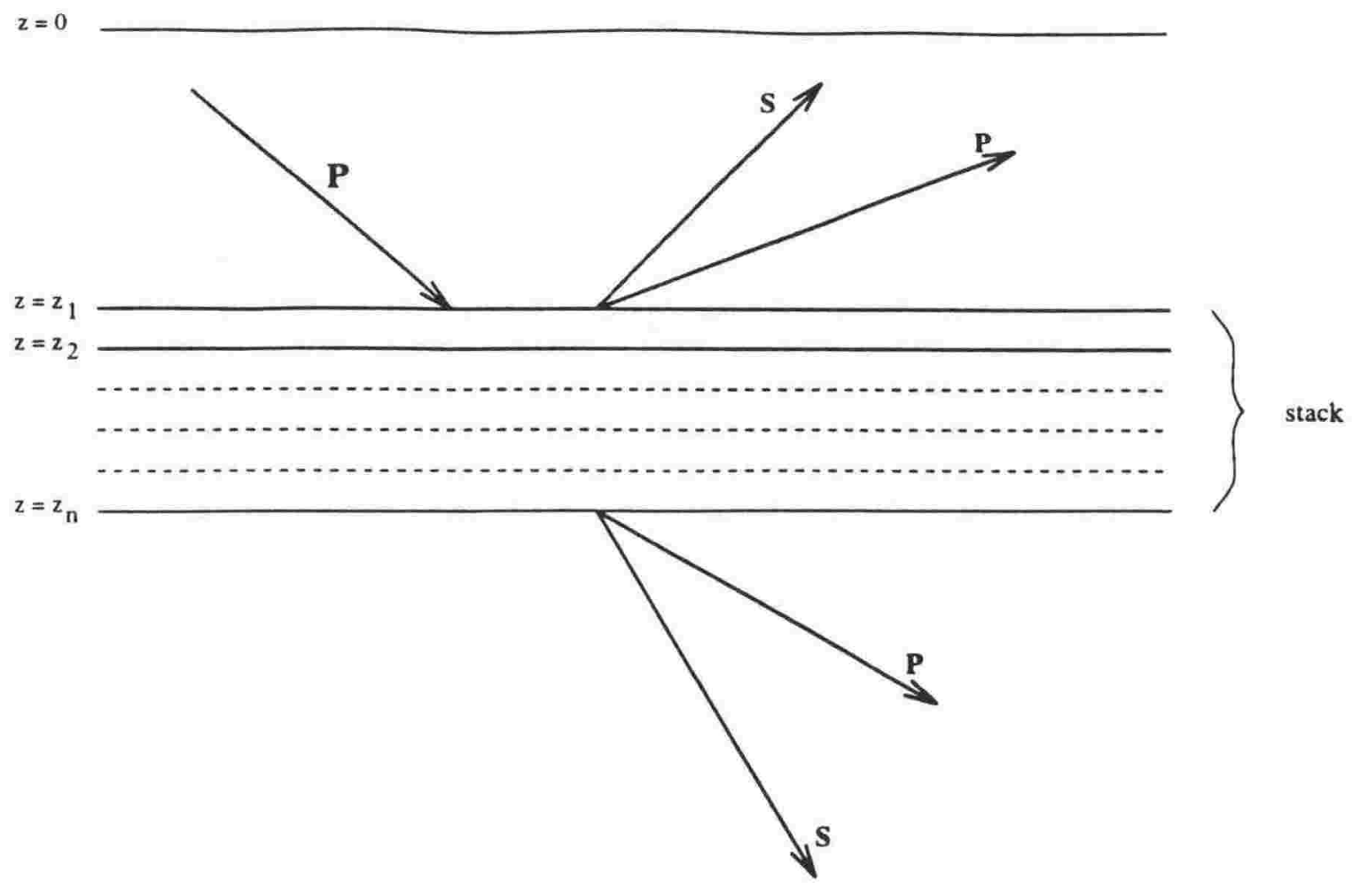

Fig. 4.1 Reflectivity of a stack of homogeneous layers

$\left(R_{P P}, R_{P S}, T_{P P}, T_{P S}\right)$ from which the reflectivities $R_{P P}, R_{P S}$ may in principle be computed.

Note that the propagator matrix $\mathbf{P}\left(z_{1}, z_{n}\right)$ and fundamental matrices $\mathbf{F}_{u}$ and $\mathbf{F}_{l}$ are functions of $\omega$ and $p$. hence the reflectivities and transmission coefficients will be functions of $\omega$ and $p$. The calculation of the response of a stratified medium must include a summation of the responses at all relevant frequencies and slownesses, and will include all possible wave types, both propagating and evanescent.

\subsection{The Wave Propagator}

Kennett uses widely the idea of a wave propagator which is similar to the stressdisplacement operator $\mathbf{P}$ but acts on the wave vector rather than on the stress-displacement vector. The fundamental matrix $\mathbf{F}$ in equation (4.5) is readily factorised into two matrices $\mathbf{D}$ and $\mathbf{Q}$, where $\mathbf{D}$ is the matrix whose columns are the eigenvectors of $\mathbf{A}$ and $\mathbf{Q}$ is a diagonal matrix 
containing the vertical phase factors $e^{ \pm i \omega \xi\left(z-z_{0}\right)}$ and $e^{ \pm i \omega \eta\left(z-z_{0}\right)}$. Thus for P-SV waves

$$
\mathbf{Q}=\left(\begin{array}{cccc}
e^{i \omega \xi\left(z-z_{o}\right)} & 0 & 0 & 0 \\
0 & e^{i \omega \eta\left(z-z_{o}\right)} & 0 & 0 \\
0 & 0 & e^{-i \omega \xi\left(z-z_{o}\right)} & 0 \\
0 & 0 & 0 & e^{-i \omega \eta\left(z-z_{o}\right)}
\end{array}\right)
$$

and

$$
\mathbf{D}=\left(\begin{array}{cccc}
\alpha p & \beta \eta & \alpha p & \beta \eta \\
\alpha \xi & -\beta \eta & -\alpha \xi & \beta p \\
2 i \omega \alpha \beta^{2} p \xi & i \omega \rho \beta\left(1-2 \beta^{2} p^{2}\right) & -2 i \omega \rho \alpha \beta^{2} p \xi & -i \omega \rho \beta\left(1-2 \beta^{2} p^{2}\right) \\
i \omega p \alpha\left(1-2 \beta^{2} p^{2}\right) & -2 i \omega \rho \beta^{3} p \eta & i \omega \rho \alpha\left(1-2 \beta^{2} p^{2}\right) & -2 i \omega \rho \beta^{3} p \eta
\end{array}\right)
$$

At depth $\mathrm{z}$, we have

$$
\mathbf{f}(z)=\mathbf{D Q}\left(z, z_{o}\right) \mathbf{w}
$$

At depth $z_{o}$ within the same medium, we have

$$
\mathbf{f}\left(z_{o}\right)=\mathrm{Dw}
$$

D is independent of $z$. Kennett(1983, p46) defines the wave-vector $\mathbf{v}$ by

$$
\mathbf{f}=\mathbf{D v}
$$

We thus have

$$
\mathbf{v}(z)=\mathbf{Q}\left(z, z_{o}\right) \mathbf{v}\left(z_{o}\right)
$$

and $Q\left(z, z_{o}\right)$ is identified as the wave-propagator. In a uniform medium, its entries represent the phase increments undergone by up- and downgoing $\mathrm{P}$ and $\mathrm{S}$ waves in travelling from $z_{o}$ to $z$. In a non-uniform medium, such as a stratified region, $\mathbf{Q}$ takes on a somewhat more complex role, as will be discussed in a later section. $\mathbf{Q}$ is easily related to $\mathbf{P}$ as follows:

$$
\begin{gathered}
\mathbf{f}(z)=\mathbf{P}\left(z, z_{o}\right) \mathbf{f}\left(z_{o}\right) \\
\operatorname{Dv}(z)=\mathbf{P}\left(z, z_{o}\right) \operatorname{Dv}\left(z_{o}\right) \\
\mathbf{v}(z)=\mathbf{D}^{-1} \mathbf{P}\left(z, z_{o}\right) \operatorname{Dv}\left(z_{o}\right)
\end{gathered}
$$

Thus

$$
\mathbf{Q}\left(z, z_{o}\right)=\mathbf{D}^{-1} \mathbf{P}\left(z, z_{o}\right) \mathbf{D}
$$

$\mathbf{Q}\left(z, z_{o}\right)$ has similar properties as $\mathbf{P}$, in particular, it obeys the chain rule:

$$
\mathbf{Q}\left(z_{A}, z_{C}\right)=\mathbf{Q}\left(z_{A}, z_{B}\right) \mathbf{Q}\left(z_{B}, z_{C}\right)
$$


An interesting way of writing (10) is by partitioning both the eigenvector matrix $\mathbf{D}$ and the wavevector $\mathbf{v}$ as follows:

$$
\mathbf{f}(z)=\left(\begin{array}{ll}
\mathbf{M}_{U} & \mathbf{M}_{D} \\
\mathbf{N}_{U} & \mathbf{N}_{D}
\end{array}\right)\left(\begin{array}{l}
\mathbf{v}_{U} \\
\mathbf{v}_{D}
\end{array}\right)
$$

where $\mathbf{M}_{U}, \mathbf{M}_{D}, \mathbf{N}_{U}, \mathbf{N}_{D}$ are $(2 \times 2)$ submatrices of $\mathbf{D}$ in the case of P-SV waves, and $D, U$ refer to up- and downgoing waves. Thus $\mathbf{M}_{U}$ acting on $\mathbf{v}_{U}$ generates displacements for upgoing waves, etc.. The matrix $\mathbf{D}$ is frequency-independent.

Equation (11) may be re-written as

$$
\mathbf{P}\left(z_{A}, z_{B}\right)=\mathbf{D Q}\left(z_{A}, z_{B}\right) \mathbf{D}^{-1}
$$

A useful interpretation of this is that when $\mathbf{P}\left(z_{A}, z_{B}\right)$ acts upon a stress-displacement vector $\mathbf{f}\left(z_{B}\right)$, $\mathbf{D}^{-1}$ first splits it up into up- and downgoing parts, $\mathbf{Q}\left(z_{A}, z_{B}\right)$ then "transfers" these parts to $z_{A}$ via the vertical phase factors, and $\mathbf{D}$ reconstitutes them back into a stress-displacement vector at $z_{A}$.

\subsection{Reflection and Transmission Matrices}

The use of propagator matrices in evaluating the response of a layered medium involves numerical difficulties which limit the application of the method under certain conditions. These difficulties arise from the terms $e^{ \pm \omega \xi\left(z-z_{0}\right)}$ and $e^{ \pm i \omega \eta\left(z-z_{0}\right)}$. In the case of imaginary $\xi$ and $\eta$, when the exponents become real (evanescent waves), these terms include exponentially growing solutions with depth, which tend to make the computation "blow up". The effect is more serious at high frequencies. An analogous effect is also encountered when very thin layers are used in the model.

Kind (1976) developed a computational procedure which eliminates the problem to a certain degree. Kennett (1983) avoids it completely by using a scheme based on reflection and transmission matrices, rather than the propagator matrices themselves. This scheme will be outlined briefly in this section.

\subsubsection{Reflection/Transmission Coefficients at a Single Interface}

The use of a matrix representation for plane waves provides an elegant scheme for evaluating the reflection/transmission coefficients at a single interface representing a first-order discontinuity in the elastic parameters of the medium. Let us take the simpler case of SH waves incident on an interface separating two homogeneous media (1) and (2) (Figure 4.2a). The stressdisplacement vectors in the two media are 


$$
\begin{aligned}
& \mathbf{f}_{1}=\mathbf{F}_{1} \mathbf{w}_{1} \\
& \mathbf{f}_{2}=\mathbf{F}_{2} \mathbf{w}_{2}
\end{aligned}
$$

The stress-displacement vector is continuous across boundaries, thus

$$
\begin{aligned}
& \mathbf{F}_{1} \mathbf{w}_{1}=\mathbf{F}_{2} \mathbf{w}_{2} \\
& \mathbf{w}_{1}=\mathbf{F}_{1}^{-1} \mathbf{F}_{2} \mathbf{w}_{2}
\end{aligned}
$$

If we impose the condition that there are no upgoing waves in medium (2), then the weighting vectors $\mathbf{w}_{1}, \mathbf{w}_{2}$ are given by:

$$
\mathbf{w}_{1}=\left(\begin{array}{c}
1 \\
R S S
\end{array}\right) w_{2}=\left(\begin{array}{c}
T S S \\
0
\end{array}\right)
$$

where RSS is the reflection coefficient and TSS is the transmission coefficient for the interface. Then the matrix equation

$$
\left(\begin{array}{c}
1 \\
R S S
\end{array}\right)=\mathbf{F}_{1}^{-1} \mathbf{F}_{2}\left(\begin{array}{c}
T S S \\
0
\end{array}\right)
$$

represents a system of 2 equations in 2 unknowns, from which RSS, TSS may be found explicitly.

For P-SV waves, the calculation is more involved because this case involves mode conversion from $P$ to $S$ in both the reflected and transmitted waves (Figure $4.2 b$ ). There are thus 4 coefficients to be found-RPP, RSP,TPP and TSP. For mode conversions we follow here the notation of Kennett (1983), where e. g. RSP denotes conversion of P to S on reflection etc. In the case of P-SV, the solution for the coefficients thus involves 4 equations in 4 unknowns. The explicit expressions for all possible reflection and transmission coefficients are given in Aki and Richards (1980. p150). It should be noted that these coefficients for a single interface are independent of trequency, but depend strongly on slowness, $p$, or equivalently on the angle of incidence $j$, thr ugh the relation $p=\frac{\sin j}{c}$, where $c$ is the velocity of the incident wave.

\subsubsection{Definition of Reflection/Transmission Matrices of a Single Interface}

Kennett (1983, p104) introduces the concept of a reflection matrix made up of reflection coefficients to describe the full reflected wavefield arising from an incident wavefield comprising both $\mathrm{P}$ and SV waves. A similar matrix is defined for transmission. These matrices are denoted by $\mathbf{R}_{D}, \mathbf{T}_{D}$ for downgoing incident waves, and $\mathbf{R}_{U}$ and $\mathbf{T}_{U}$ for upgoing incident waves (incidence from below). For SH wave incidence, the matrices are just the individual reflection and transmission coefficients. 


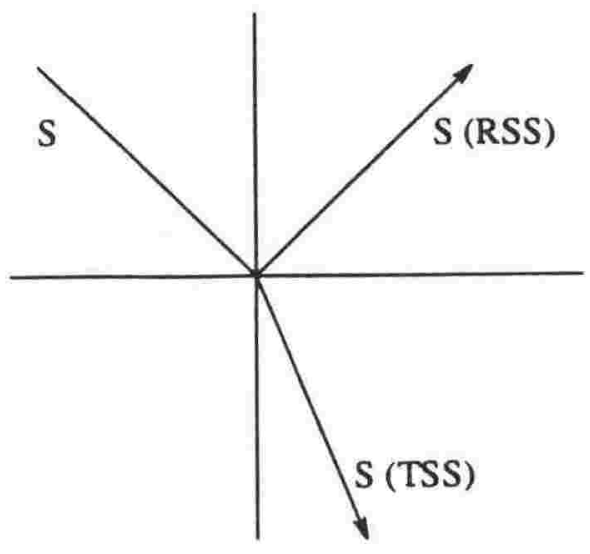

(a)

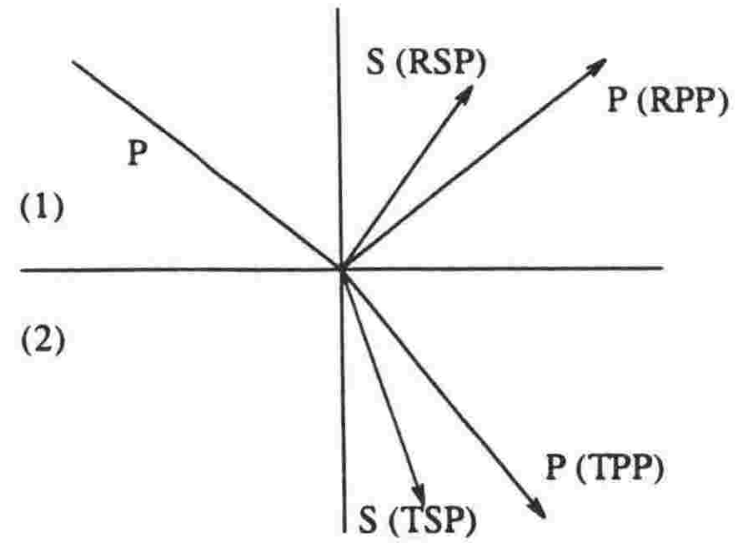

(b)

Fig. 4.2 (a) Reflection and transmission coefficients for an incident $S$-wave (b) Reflection and transmission coefficients for an incident $P$-wave

For P-SV waves:

$$
\begin{aligned}
\mathbf{R}_{D} & =\left(\begin{array}{ll}
R P P_{D} & R P S_{D} \\
R S P_{D} & R S S_{D}
\end{array}\right) \\
\cdot & \\
\mathbf{T}_{D} & =\left(\begin{array}{ll}
T P P_{D} & T P S_{D} \\
T S P_{D} & T S S_{D}
\end{array}\right)
\end{aligned}
$$

so that

$$
\left(\begin{array}{c}
P_{U 1} \\
S_{U 1}
\end{array}\right)=\left(\begin{array}{ll}
R P P_{D} & R P S_{D} \\
R S P_{D} & R S S_{D}
\end{array}\right)\left(\begin{array}{c}
P_{D 1} \\
S_{D 1}
\end{array}\right)
$$


and

$$
\left(\begin{array}{c}
P_{D 1} \\
S_{D 1}
\end{array}\right)=\left(\begin{array}{ll}
T P P_{D} & T P S_{D} \\
T S P_{D} & T S S_{D}
\end{array}\right)\left(\begin{array}{l}
P_{D 1} \\
S_{D 1}
\end{array}\right)
$$

where subscripts $U, D$ refer to up- and downgoing waves respectively, and subscripts 1,2 refer to media (1) and (2). Thus, for instance, the first equation represented by equation (4.12a) says that the upgoing P wave in medium (1) is a linear combination of the PP reflected wave and the SP reflected wave.

In terms of wavevectors, the previous equations may be expressed as

$$
\begin{aligned}
& \mathbf{v}_{U 1}=\mathbf{R}_{D} \mathbf{v}_{D 1} \\
& \mathbf{v}_{D 2}=\mathbf{T}_{D} \mathbf{v}_{D 1}
\end{aligned}
$$

Similarly, for incidence from below the interface

$$
\begin{aligned}
& \mathbf{v}_{D 2}=\mathbf{R}_{U} \mathbf{v}_{U 2} \\
& \mathbf{v}_{U 1}=\mathbf{T}_{U} \mathbf{v}_{U 2}
\end{aligned}
$$

The concept of reflection and transmission matrices is generalized to relate the whole wave vectors below and above an interface as follows:

$$
\left(\begin{array}{l}
\mathbf{v}_{U 1} \\
\mathbf{v}_{D 1}
\end{array}\right)=\mathbf{Q}(1,2)\left(\begin{array}{l}
\mathbf{v}_{U 2} \\
\mathbf{v}_{D 2}
\end{array}\right)
$$

where the wave propagator $\mathbf{Q}$ here plays the special role of transferring a wave vector across an interface. It is useful to partition $Q(1,2)$ into $4(2 \times 2)$ submatrices as follows:

$$
\left(\begin{array}{l}
\mathbf{v}_{U 1} \\
\mathbf{v}_{D 1}
\end{array}\right)=\left(\begin{array}{ll}
\mathbf{Q}_{U U} & \mathbf{Q}_{U D} \\
\mathbf{Q}_{D U} & \mathbf{Q}_{D D}
\end{array}\right)\left(\begin{array}{l}
\mathbf{v}_{U 2} \\
\mathbf{v}_{D 2}
\end{array}\right)
$$

and by relating the equations (4.13) with the equations in (4.14) it is possible to write the matrix $\mathbf{Q}(1,2)$ completely in terms of reflection and transmission matrices, thus:

$$
\left(\begin{array}{c}
\mathbf{v}_{U 1} \\
\mathbf{v}_{D 1}
\end{array}\right)=\left(\begin{array}{cc}
\mathbf{T}_{U}-\mathbf{R}_{D} \mathbf{T}_{D}^{-1} \mathbf{R}_{U} & \mathbf{R}_{D} \mathbf{T}_{D}^{-1} \\
-\mathbf{T}_{D}^{-1} \mathbf{R}_{U} & \mathbf{T}_{D}^{-1}
\end{array}\right)\left(\begin{array}{c}
\mathbf{v}_{U 2} \\
\mathbf{v}_{D 2}
\end{array}\right)
$$

\subsubsection{Overall Reflection/Transmission Matrices of a Stratified Region}

The reflection and transmission matrices for a stratified region $\mathrm{AC}$ embedded in two uniform half-spaces (1) and (2) as in Figure 4.3, follows much the same procedure as for a single interface. The matrices for the whole region $A C$ are written as $\mathbf{R}_{D}^{A C}, \mathbf{T}_{D}^{A C}, \mathbf{R}_{U}^{A C}$ and $\mathbf{T}_{U}^{A C}$ for 
incident waves from above and below. The wave propagator matrix relating the wave vectors at $z_{A}$ and $z_{C}$ is given by

$$
\mathbf{Q}\left(z_{A}, z_{C}\right)=\left(\begin{array}{cc}
\mathbf{T}_{U}^{A C}-\mathbf{R}_{D}^{A C}\left(\mathbf{T}_{D}^{A C}\right)^{-1} \mathbf{R}_{U}^{A C} & \mathbf{R}_{D}^{A C}\left(\mathbf{T}_{D}^{A C}\right)^{-1} \\
-\left(\mathbf{T}_{D}^{A C}\right)^{-1} \mathbf{R}_{U}^{A C} & \left(\mathbf{T}_{D}^{A C}\right)^{-1}
\end{array}\right)
$$

For a wave incident from below, the matrix is transformed thus:

$$
\mathbf{Q}\left(z_{C}, z_{A}\right)=\left(\begin{array}{cc}
\left(\mathbf{T}_{U}^{A C}\right)^{-1} & -\left(\mathbf{T}_{U}^{A C)^{-1}} \mathbf{R}_{D}^{A C}\right. \\
\mathbf{R}_{U}^{A C}\left(\mathbf{T}_{U}^{A C}\right)^{-1} & \mathbf{T}_{D}^{A C}-\mathbf{R}_{U}^{A C}\left(\mathbf{T}_{U}^{A C}\right)^{-1} \mathbf{R}_{D}^{A C}
\end{array}\right)
$$

The step of recasting the wave propagator entirely in terms of reflection and transmission matrices is central to Kennett's method of calculating the response of a stratified medium in a way which avoids the numerical difficulties inherent in the use of the propagator matrices $\mathbf{P}$.

Note that within any uniform layer, there is no reflection and the nature of $\mathbf{Q}$ reverts to a

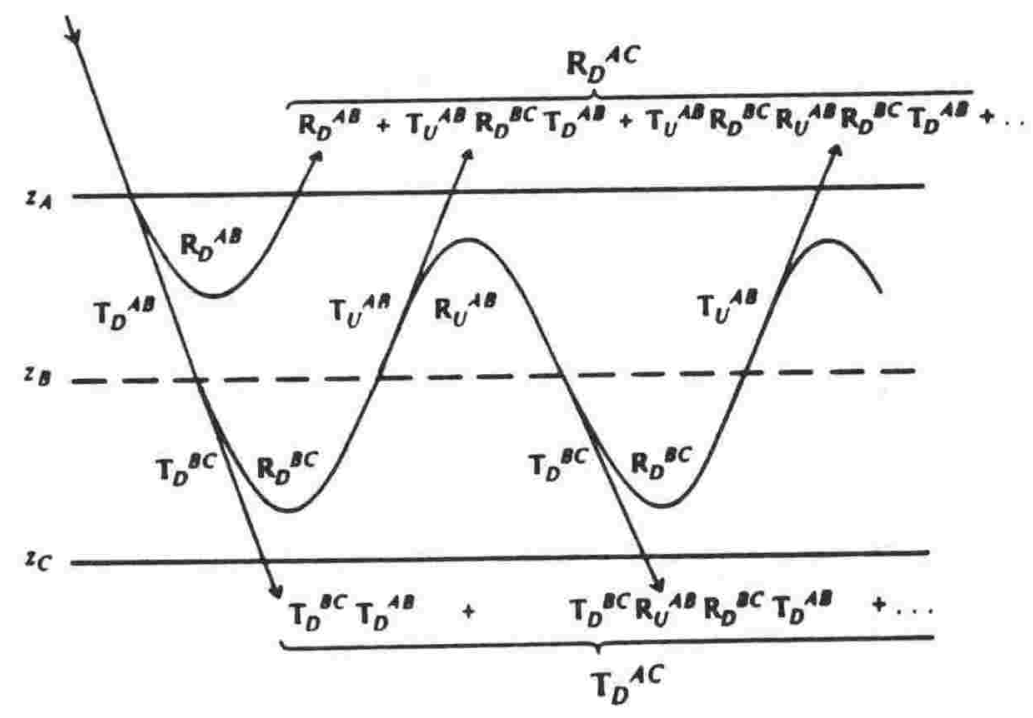

Fig.4.3 Reflection and transmission matrices for a stratified region 
simple transmission via the phase factors. Thus if D and E are two points within the same uniform layer,

$$
\begin{gathered}
\mathbf{Q}\left(z_{E}, z_{F}\right)=\left(\begin{array}{cc}
\mathbf{T}_{U}^{E F} & 0 \\
0 & \left(\mathbf{T}_{D}^{E F}\right)^{-1}
\end{array}\right) \\
=\left(\begin{array}{cccc}
e^{i \omega \xi\left(z_{E}-z_{F}\right)} & 0 & 0 & 0 \\
0 & e^{i \omega \eta\left(z_{E}-z_{F}\right)} & 0 & 0 \\
0 & 0 & e^{-i \omega \xi\left(z_{E}-z_{F}\right)} & 0 \\
0 & 0 & 0 & e^{-i \omega \eta\left(z_{E}-z_{F}\right)}
\end{array}\right)
\end{gathered}
$$

\subsection{A Stack of Uniform Layers}

\subsubsection{A Split Region}

Consider first a region $\left(z_{A}, z_{C}\right)$ as in the previous section, split up further by the introduction of a new interface $z_{B}$ where $z_{A} \leq z_{B} \leq z_{C}$ (Figure 4.3). By the chain rule for wave propagators

$$
\mathbf{Q}\left(z_{A}, z_{C}\right)=\mathbf{Q}\left(z_{A}, z_{B}\right) \mathbf{Q}\left(z_{B}, z_{C}\right)
$$

where each of $\mathbf{Q}\left(z_{A}, z_{B}\right)$ and $\mathbf{Q}\left(z_{B}, z_{C}\right)$ has the representation (15). Multiplying out the respective matrices results, with some manipulation, in the following addition rules for the split structure:

For downward incidence

$$
\begin{gathered}
\mathbf{T}_{D}^{A C}=\mathbf{T}_{D}^{B C}\left[\mathbf{I}-\mathbf{R}_{U}^{A B} \mathbf{R}_{D}^{B C}\right]^{-1} \mathbf{T}_{D}^{A B} \\
\mathbf{R}_{D}^{A C}=\mathbf{R}_{D}^{A B}+\mathbf{T}_{U}^{A B} \mathbf{R}_{D}^{B C}\left[\mathbf{I}-\mathbf{R}_{U}^{A B} \mathbf{R}_{D}^{B C}\right]^{-1} \mathbf{T}_{D}^{A B}
\end{gathered}
$$

For upward incidence

$$
\begin{gathered}
\mathbf{T}_{U}^{A C}=\mathbf{T}_{U}^{A B}\left[\mathbf{I}-\mathbf{R}_{D}^{B C} \mathbf{R}_{U}^{A B}\right]^{-1} \mathbf{T}_{U}^{B C} \\
\mathbf{R}_{U}^{A C}=\mathbf{R}_{U}^{B C}+\mathbf{T}_{D}^{B C} \mathbf{R}_{U}^{A B}\left[\mathbf{I}-\mathbf{R}_{D}^{B C} \mathbf{R}_{U}^{A B}\right]^{-1} \mathbf{T}_{U}^{B C}
\end{gathered}
$$

where I is the identity matrix.

These rules are central to Kennett's scheme for working out the overall reflectivity and transmissivity of a stack of uniform layers. In particular, the matrix inverses of the type 
$\left[\mathbf{I}-\mathbf{R}_{U}^{A B} \mathbf{R}_{D}^{B C}\right]^{-1}$ are called reverberation operators and have a key role in the interpretation of the wavefield as a sum of multiple reflections and transmissions within successive layers. This is achieved by expressing the inverse as a series matrix expansion:

$$
\left[\mathbf{I}-\mathbf{R}_{U}^{A B} \mathbf{R}_{D}^{B C}\right]^{-1}=\mathbf{I}+\mathbf{R}_{U}^{A B} \mathbf{R}_{D}^{B C}+\mathbf{R}_{U}^{A B} \mathbf{R}_{D}^{B C} \mathbf{R}_{U}^{A B} \mathbf{R}_{D}^{B C}+\cdots
$$

The increasing order of terms represents wavefields made up of an increasing number of internal multiples, which can be retained or suppressed at will by specifying the maximum order in the expansion. Thus the simplest approximation is

$$
\left[\mathbf{I}-\mathbf{R}_{U}^{A B} \mathbf{R}_{D}^{B C}\right]^{-1}=\mathbf{I}
$$

This, from (17a) and (17b), produces the overall reflection and transmission matrices

$$
\begin{gathered}
\mathbf{R}_{D}^{A C}=\mathbf{R}_{D}^{A B}+\mathbf{T}_{U}^{A B} \mathbf{R}_{D}^{B C} \mathbf{T}_{D}^{A B} \\
\mathbf{T}_{D}^{A C}=\mathbf{T}_{D}^{B C} \mathbf{T}_{D}^{A B}
\end{gathered}
$$

Looking at Figure 4.3, this represents waves which have been transmitted down through $A B$, reflected once by region $\mathrm{BC}$ and transmitted upwards by region $\mathrm{AB}$. The compound terms in the expressions should be read from right to left.

The next degree of complexity is represented by retaining the second term in the expansion, thus we get

$$
\begin{gathered}
\mathbf{R}_{D}^{A C}=\mathbf{R}_{D}^{A B}+\mathbf{T}_{U}^{A B} \mathbf{R}_{D}^{B C} \mathbf{T}_{D}^{A B}+\mathbf{T}_{U}^{A B} \mathbf{R}_{D}^{B C} \mathbf{R}_{U}^{A B} \mathbf{R}_{D}^{B C} \mathbf{T}_{D}^{A B} \\
\mathbf{T}_{D}^{A C}=\mathbf{T}_{D}^{B C} \mathbf{T}_{D}^{A B}+\mathbf{T}_{D}^{B C} \mathbf{R}_{U}^{A B} \mathbf{R}_{D}^{B C} \mathbf{T}_{D}^{A B}
\end{gathered}
$$

This represents waves which have also been reflected down by the region $A B$ before being reflected back up by $\mathrm{BC}$, and so on. Similar interpretations apply for the transmission operator. The full inverse yields a full response with all possible reverberations within layers. It should be remembered that the off-diagonal terms in the reflection and transmission matrices (12) ensure that mode conversions between $\mathrm{P}$ and $\mathrm{S}$ wave types are fully represented in the scheme.

\subsubsection{The Recursive Scheme for the Overall Reflection and Transmission Matrices}

The addition rules (17) form the basis of the recursive scheme by which Kennett's method systematically builds up the overall reflection and transmission matrices for a stack of any number of uniform layers (Kennett, 1983 Chapter 6). The scheme is carried out in progressive stages of 2 steps each, starting at the base of the layering, which is usually the boundary with the lower halfspace (Figure 4.4)

(i) The interface matrices $\mathbf{R}_{D}, \mathbf{R}_{U}, \mathbf{T}_{D}, \mathbf{T}_{U}$ at the base of layer $n$ are 'propagated' to the top 
of layer $n$ by a simple transmission of the form (16), recognizing that there is no reflection within the layer. The expressions are

$$
\begin{gathered}
\mathbf{R}_{D}\left(n_{\text {top }}\right)=\mathbf{T}_{U}^{z_{n} z_{n+1}} \mathbf{R}_{D}\left(n_{\text {bot }}\right) \mathbf{T}_{D}^{z_{n} z_{n+1}} \\
\mathbf{T}_{D}\left(n_{\text {top }}\right)=\mathbf{T}_{D}\left(n_{\text {bot }}\right) \mathbf{T}_{D}^{z_{n} z_{n+1}} \\
\mathbf{R}_{U}\left(n_{\text {top }}\right)=\mathbf{R}_{U}\left(n_{\text {bot }}\right) \\
\mathbf{T}_{U}\left(n_{\text {top }}\right)=\mathbf{T}_{U}^{z_{n} z_{n+1}} \mathbf{T}_{U}\left(n_{\text {bot }}\right)
\end{gathered}
$$

where the elements of $\mathbf{T}_{D}^{z_{n} z_{n+1}}$ and $\mathrm{T}_{U}^{z_{n} z_{n+1}}$ are simply phase shifts corresponding to the thickness of the layer $n$, and e.g. $\mathbf{R}_{D}\left(n_{\text {bot }}\right)$ is equivalent to the interface matrix of the level $z_{n+1}$.

The reflection matrices at the bottom of the next layer $(n-1)$ are calculated using again the addition rules (17), and effectively treating the boundary as an infinitesimal extra layer with reflection and transmission matrices equal to the interface matrices. Thus

$$
\begin{gathered}
\mathbf{R}_{D}\left(n-1_{\text {bot }}\right)=\mathbf{R}_{D}\left(z_{n}\right)+\mathbf{T}_{U}\left(z_{n}\right) \mathbf{R}_{D}\left(n_{\text {top }}\right)\left[\mathbf{I}-\mathbf{R}_{U}\left(z_{n}\right) \mathbf{R}_{D}\left(n_{\text {top }}\right)\right]^{-1} \mathbf{T}_{D}\left(z_{n}\right) \\
\mathbf{T}_{D}\left(n-1_{\text {bot }}\right)=\mathbf{T}_{D}\left(n_{\text {top }}\right)\left[\mathbf{I}-\mathbf{R}_{U}\left(z_{n}\right) \mathbf{R}_{D}\left(n_{\text {top }}\right)\right]^{-1} \mathbf{T}_{D}\left(z_{n}\right) \\
\mathbf{R}_{U}\left(n-1_{\text {bot }}\right)=\mathbf{R}_{U}\left(n_{\text {top }}\right)+\mathbf{T}_{D}\left(n_{\text {top }}\right) \mathbf{R}_{U}\left(z_{n}\right)\left[\mathbf{I}-\mathbf{R}_{D}\left(n_{\text {top }}\right) \mathbf{R}_{U}\left(z_{n}\right)\right]^{-1} \mathbf{T}_{U}\left(n_{\text {top }}\right) \\
\mathbf{T}_{U}\left(n-1_{\text {bot }}\right)=\mathbf{T}_{U}\left(z_{n}\right)\left[\mathbf{I}-\mathbf{R}_{D}\left(n_{\text {top }}\right) \mathbf{R}_{U}\left(z_{n}\right)\right]^{-1} \mathbf{T}_{U}\left(n_{\text {top }}\right)
\end{gathered}
$$

The additions are repeated for every successive layer and interface to the top of the stack of layers. Thus it is evident that we only need single interface matrices, such as (12), to build up the final result. Note that the only terms which are frequency dependent are the transmission terms $\mathbf{T}_{D}^{z_{i} z_{i+1}}$ and $\mathbf{T}_{U}^{z_{i} z_{i+1}}$. The interface matrices are frequency independent, therefore in the computation they need only be calculated once for any given slowness, and can be re-used for every frequency.

\subsection{The Response of a Medium to a Source}

The matrix addition rules make it possible to divide the whole structure from the free surface to the lower half-space into regions, which may themselves be stratified, and to calculate the response of each region to upward and downward incident wavefields according to the degree 


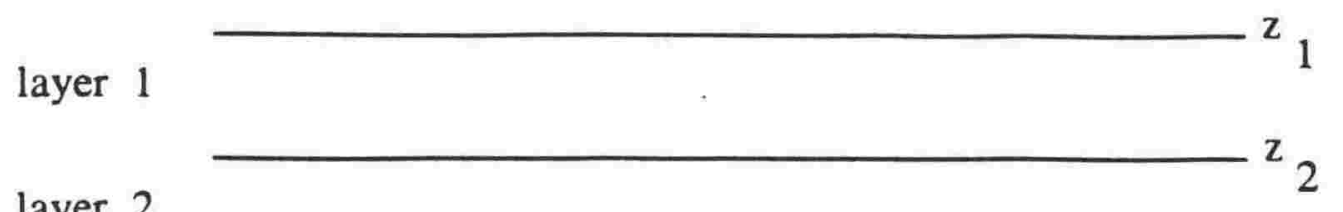

layer 2

layer $\mathrm{n}-1$

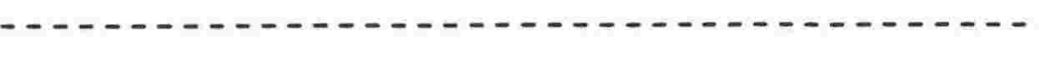

layer $n$

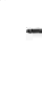

layer $\mathrm{n}$

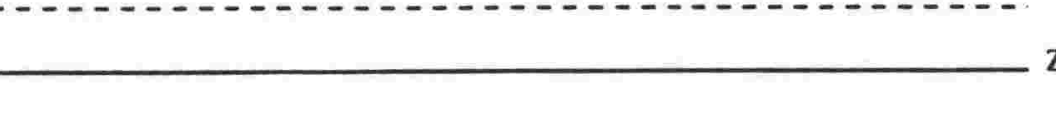

half space

Fig. 4.4 Layering system used in recursive scheme (Kennett, 1983)

of complexity required. In particular, for a source at depth $z_{s}$ below the surface, an interface is introduced at $z_{s}$ and the radiation from the source is considered in two separate parts - upgoing radiation incident on the region above the source, and downgoing radiation incident on the region below the source (Figure 4.5).

The radiation from the source is made up of a discontinuity at depth $z_{s}$ of the stressdisplacement vector (Kennett,1983 p95). This radiation is azimuth dependent, according to the orientation and nature of the source, and depends on the azimuthal order $m$ of the cylindrical functions which constitute the total wavefield as a sum of cylindrical waves (see Section 4.9). The discontinuity in the stress-displacement vector is denoted by

$$
\mathbf{S}\left(p, m, z_{s}, \omega\right)=\left[\mathbf{f}\left(p, m, z_{s}, \omega\right)\right]
$$

where the square brackets mean a jump in the function.

For a point, double-couple source the jumps in the 3 displacement components and the 3 traction components are explicitly dependent on the components $M_{i j}(i, j=1 . .3)$ of the moment tensor for the source, and on the order $m$ of the cylindrical functions, where $m=0, \pm 1, \pm 2$. These 
expressions are given in Kennett (1983, p95).

The jump in the stress-displacement vector is converted to a jump in wave-vectors $\Sigma\left(p, m, z_{s}, \omega\right)$ by the operation of the inverse eigenvector matrix (equation (4.10):

$$
\Sigma\left(p, m, z_{s}, \omega\right)=\mathbf{D}^{-1}\left(p, z_{s}\right) \mathbf{S}\left(p, m, z_{s}, \omega\right)=\left[v\left(p, m, z_{s}, \omega\right)\right]
$$

and the source vector $\Sigma$ is written in terms of up and downgoing radiation as

$$
\Sigma=\left(\begin{array}{c}
-\Sigma_{U} \\
\Sigma_{D}
\end{array}\right)
$$

\subsection{The Surface Displacement}

We are ultimately interested in having an expression for the total displacement at the free surface, $z=0$, due to a source at depth, and at some range from the source. It should be kept in mind that the basic transformation that converts the wave vector to a stress-displacement vector is via the eigenfunction matrix $\mathbf{D}$

$$
\mathfrak{f}(z)=\operatorname{Dv}(z)
$$

and that with the partitioning of $\mathbf{D}$ as in (10a), it is the submatrices $\mathbf{M}_{U}$ and $\mathbf{M}_{D}$ that generate the displacement components. The treatment of Kennett is very general and rigorous, but it is enough here to give the general result for the free surface displacement $\mathbf{u}$ and its interpretation. Following Kennett (1988), this may be written as

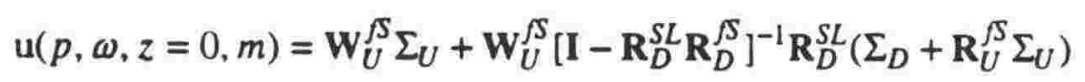

where $\mathrm{L}$ represents the base of the stratification, $\mathrm{S}$ represents the level of the source depth and $\mathrm{F}$ represents the free surface (see Figure 4.5).

The term $\mathbf{W}_{U}^{\mathcal{S}}$ is actually a displacement matrix ( $(2 \times 2)$ for P-SV and $(1 \times 1)$ for SH) which contains the actual surface displacements generated by incident upgoing $\mathrm{P}$ and $\mathrm{S}$ waves. It behaves essentially like a transmission matrix coupled with the displacement-generating submatrices of matrix $\mathbf{D}$ in (10a). The actual form of $\mathbf{W}_{U}^{\mathcal{S}}$ is

$$
\mathbf{W}_{U}^{\mathcal{S}}=\left(\mathbf{M}_{U}+\mathbf{M}_{D} \tilde{\mathbf{R}}\right) \mathbf{T}_{U}^{f S}
$$

where $\tilde{\mathbf{R}}$ is the actual reflection matrix of the free surface, giving the amplification effects of reflection, and $\mathbf{T}_{U}^{\mathcal{S}}$ is the transmission matrix which carries the wave vector from the source up to the surface. 


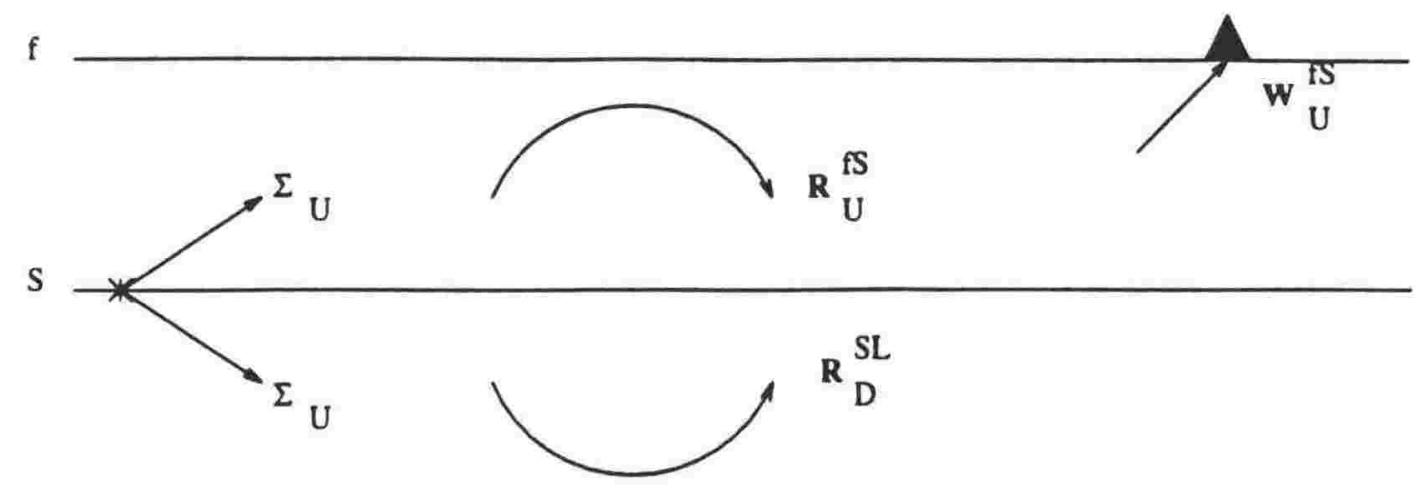

L

Fig. 4.5 Response of a medium to a source

The term $\mathbf{R}_{U}^{\mathcal{S}}$ represents the response of the structure between the source and the free surface, and can be expanded, using the addition rules (17) in a way similar to that used in the recursive scheme:

$$
\mathbf{R}_{Y^{\prime}}^{j S}=\mathbf{R}_{U}^{O S}+\mathbf{T}_{D}^{O S} \tilde{\mathbf{R}}\left[\mathbf{I}-\mathbf{R}_{D}^{O S} \tilde{\mathbf{R}}\right]^{1} \mathbf{T}_{U}^{O S}
$$

where $O$ refers to a level just below the surface. This expansion thus allows the possibility of ignoring the free surface reflections, if so desired.

The term $\mathbf{R}_{D}^{S L}$ represents the response of the region between the source and the base of the stratification, or the top of the half-space.

Thus, referring to Figure 4.5, equation (4.18) has a clear physical interpretation in terms of all possible propagation processes making up the response. The first term in the equation deals with energy transmitted upwards from the source to the surface. The second term includes both the energy radiated downwards and reflected back up from the region SL, as well as the energy radiated upwards and reflected back down from the region fS (via the term $\mathbf{R}_{U}^{f S} \Sigma_{U}$ ). The 
reverberation operator allows the fullest possible interaction between the upper and lower regions. however by truncating it appropriately, the reverberations may be suppressed.

The actual computation is systematic, using successive applications of the recursive scheme described in Section 4.6.2, to evaluate the various reflection and transmission matrices. For example, $\mathbf{R}_{D}^{S L}$ is first constructed by starting at $z_{L}$ and working upwards through the layers to $z_{S}$. $\mathbf{R}_{U}^{f S}$ and $\mathbf{T}_{U}^{f S}$ (hence $\mathbf{W}_{U}^{f S}$ ) are similarly constructed by starting at the free surface and working downwards to $z_{S}$. The reverberation operator is fully inverted or approximated to I or the specified number of terms, according to the complexity of the response required.

\subsection{Recovery of the Time-Domain Response}

The calculation of $\mathbf{u}(p, \omega, z=0, m)$ is carried out for a set of frequencies $\omega$ within a specified range, and for a set of slowness values $p$. The total wavefield at a given frequency is the superposition of waves of all slownesses, and the spectrum of the final seismogram is a set of such wavefields at the desired set of frequencies. This section will briefly describe how the final summations are carried out.

\subsubsection{Cylindrical Wave Functions}

A natural frame of reference for describing the wavefield at some distance from a point source is the cylindrical coordinate system $(r, \phi, z)$, where $r$ is horizontal distance in the direction of propagation, and $\phi$ is the horizontal azimuth. Following Aki and Richards (1980, p398) the displacement part of the displacement-stress vector at the free surface, and distance $r$ from the source, may be written as follows:

For P-SV waves

$$
\mathbf{u}(r, \phi, \omega, k)=\sum_{m=-\infty}^{+\infty} k\left[u_{r}(k, m, z=0, \omega) \mathbf{S}_{k}^{m}-i u_{z}(k, m, z=0, \omega) \mathbf{R}_{k}^{m}\right]
$$

For $\mathrm{SH}$ waves

$$
\mathbf{u}(r, \phi, \omega, k)=\sum_{m=-\infty}^{+\infty} k u_{t}(k, m, z=0, \omega) \mathbf{T}_{k}^{m}
$$

where $u_{z}, u_{r}, u_{t}$ are the vertical, radial and transverse components of displacement. The functions $\mathbf{S}_{k}^{m}, \mathbf{R}_{k}^{m}, \mathbf{T}_{k}^{m}$ are the horizontal wavefunctions of order $m$ defined by 


$$
\begin{gathered}
\mathbf{S}_{k}^{m}=\frac{1}{k} \frac{\partial \mathbf{Y}_{k}^{m}}{\partial r} \hat{r}+\frac{1}{k r} \frac{\partial \mathbf{Y}_{k}^{m}}{\partial \phi} \hat{\phi} \\
\mathbf{R}_{k}^{m}=-\mathbf{Y}_{k}^{m}(r, \phi) \hat{z} \\
\mathbf{T}_{k}^{m}=\frac{1}{k r} \frac{\partial \mathbf{Y}_{k}^{m}}{\partial \phi} \hat{r}-\frac{1}{k} \frac{\partial \mathbf{Y}_{k}^{m}}{\partial r} \hat{\phi} \\
\mathbf{Y}_{k}^{m}(r, \phi)=\mathbf{J}_{m}(k, r) e^{i m \phi}
\end{gathered}
$$

where $\mathrm{J}_{m}(k, r)$ is the Bessel function of order $m$.

Equations (19) state that the wavefield is a linear superposition of cylindrical waves, in which the coefficients of the cylindrical wavefunctions are precisely the displacement components at the free surface, found for the stratified medium as in the previous sections. It is preferable to make a change of variable from $k$ to $p$, the horizontal slowness, using $k=\omega p$. Also, equations (4.19) are simplified by using the approximate relations:

$$
\begin{gathered}
\mathbf{R}^{m}(\omega p r, \phi)=\hat{z} \mathbf{J}_{m}(\omega p r) e^{i m \phi} \\
\mathbf{S}^{m}(\omega p r, \phi)=\hat{r} \mathbf{J}_{m-1}(\omega p r) e^{i m \phi} \\
\mathbf{T}^{m}(\omega p r, \phi)=-\hat{\phi} \mathbf{J}_{m-1}(\omega p r) e^{i m \phi}
\end{gathered}
$$

These approximations apply in the far-field. Close to the source, the expressions would contain terms in $\frac{1}{\omega p r}$, which die down rapidly with distance $r$.

\subsubsection{Recovery of Displacements in the Time-Domain}

The vertical, radial and transverse displacements as functions of time may be recovered fully in theory by integrating over all values of $p$ from 0 to $\infty$ and over all values of $\omega$ from $-\infty$ to $+\infty$, the latter integration being, in effect, a Fourier transform from the frequency to the time domain. Using the approximations (20), the total surface displacement may be written as

$$
\mathbf{u}(r, \phi, t)=\frac{1}{2 \pi} \int_{-\infty}^{\infty} \omega^{2} e^{-i \omega t} d \omega \sum_{m=-\infty}^{\infty} \int_{0}^{\infty} p\left[u_{r} \mathbf{J}_{m-1}(\omega p r)+i u_{z} \mathbf{J}_{m}(\omega p r)-u_{t} \mathbf{J}_{m}(\omega p r)\right] e^{i m \phi} d p
$$

The azimuthal dependence is associated only with the directivity of the source. In the case of a double-couple mechanism, the contributions from the angular orders are restricted to $|m| \leq 2$, in other words, there is a maximum of five terms in the summation over $m$.

In practice, the integral over $p$ is carried out as a numerical integration over a finite range of $p$ values. The range is chosen to include the propagation phenomena which are of interest in the 
final seismogram, remembering that $e . g . p>\frac{1}{\alpha}$ will make $\mathrm{P}$ waves go evanescent in the layer $\mathrm{pf}$ $\mathrm{P}$-wave velocity $\alpha$. According to the $\mathrm{P}$ and $\mathrm{S}$ wave velocities used in the model, the range of slownesses can even be "tailored" to simulate phases of main interest, e.g. $\mathrm{P}_{\mathrm{n}}$. The $p$ interval used for the numerical integration needs to be small enough to give adequate sampling of the integrand in (21), which has an oscillatory character. The higher the frequency, the finer the sampling needs to be.

Once the summations over $p$ have been completed at each value of $m$ between -2 and +2 , the summation over $m$ is carried out. The final step is the summation over $\omega$, which is in practice carried out by an FFT. This last step, done individually for the vertical, radial and transverse components, gives the 3-component synthetic seismogram.

\subsection{Allowance for Attenuation}

In a dissipative medium, in which energy losses during propagation occur due to anelasticity of the medium, it can be shown (Kennett, 1983) that the effects of such attenuation can be represented by the use of complex wave velocities, which are frequency-dependent. For weak attenuation, the $\mathrm{P}$ and $\mathrm{S}$ wave velocities are replaced by

$$
\begin{aligned}
& \bar{\alpha}(\omega)=\alpha_{1}\left[1+\frac{1}{\pi} \frac{1}{Q_{\alpha}} \ln \left(\frac{\omega}{2 \pi}\right)-i\left(\frac{1}{2 Q_{\alpha}}\right) \operatorname{sgn}(\omega)\right] \\
& \bar{\beta}(\omega)=\beta_{1}\left[1+\frac{1}{\pi} \frac{1}{Q_{\beta}} \ln \left(\frac{\omega}{2 \pi}\right)-i\left(\frac{1}{2 Q_{\beta}}\right) \operatorname{sgn}(\omega)\right]
\end{aligned}
$$

where

$\alpha_{1}, \beta_{1}$ are standard reference values, normally taken to be the $\mathrm{P}$ and $\mathrm{S}$ wave velocities at $1 \mathrm{~Hz}$;

$\frac{1}{Q_{\alpha}}, \frac{1}{Q_{\beta}}$ are the loss factors, which are in general smaller than 0.01 for weak attenuation, and may be assumed constant;

$\operatorname{sgn}(\omega)= \pm 1$, according to the sign of $\omega$. 


\subsection{Practical Application of the Reflectivity Method}

The modelling, in practice, of real earthquake data with synthetic seismograms involves, first of all, considerable effort in the search for the optimum parameters to be used in the computation, that provide the best compromise between the amount of information to be contained in the synthetic seismogram, the quality of the synthetic seismogram and the computing time that can be afforded. The latter increases drastically with complexity of the model and of the response, the number of samples in the Fourier transforms, the frequency range and the number of slowness values in the numerical integration. If the computation time becomes prohibitively large, then some information has to be sacrificed in any one run of the program. Kennett (1983) achieves this by structuring the build-up of the medium's response in a series of systematic approximations to the total wavefield. The user is faced with a wide range of options, but this is an advantage in the sense that the modelling can be selective as regards the exact phases being synthesised, and this facilitates greatly the interpretation of the theoretical seismograms, which can rapidly become more and more complex.

Following are the main options available in the program used in this study (Kennett, 1988) and the criteria used in choosing some of the parameters:

(i) Choice of wavetype: Since the up- and down-going radiation terms at the source can be split into the contributions from $\mathrm{P}$ and $\mathrm{S}$ wave types, it is possible to start off with either or both wavetypes at the source. Simiiarly, the elements of the surface displacement matrix can be associated with specific wavetypes, so that the same choice can be made at the receiver. This allows the modelling and identification of P-S and S-P conversions. The options are:
$\mathrm{P}$ at source, $\mathrm{P}$ at receiver
$S$ at source. $S$ at receiver
$P$ at source, $S$ at receiver
$\mathrm{S}$ at source, $\mathrm{P}$ at receiver
$P, S$ at source, $P$ at receiver
$P, S$ at source, $S$ at receiver
$\mathrm{P}, \mathrm{S}$ at source, $\mathrm{P}, \mathrm{S}$ at receiver (full response)

(ii) Complexity of response: The amount of reverberation within the model can be controlled both by limiting, the reverberations within individual layers, as well as for the structure as a whole. Kennett (1983) introduces a further possibility of restricting the total wavefield by defining a "separation level" $z_{J}$ and splitting the total response into that of the zones above and below $z_{J}$ (Figure 4.6). The physical significance of the separation level is often a division into crust and mantle regions, with the source being above the mantle. Thus one can investigate 
separately the dominant response of ,say, the mantle structure, or of the crustal waveguide. Free surface reflections and surface conversions between wavetypes play a important part in the total response, and these can also be included or suppressed at will. The main options are:

a. No reflections, primary reflections only, or full reverberation within each individual layer;

b. The response of the shallow zone (above the level $z_{J}$ may include no reflections, the primary reflection only, or multiple reflections between the free surface and the lower boundary of the zone;

c. The response from the deeper structure (below level $z_{J}$ may be evaluated once only or may be returned more than once, after interaction with one, or multiple reflections/conversions at the free surface. Thus one can model the fullest reverberation response within the crustal waveguide, while only the primary reflectivity response of the upper mantle is included.

The free surface amplification at the receiver may also be included or excluded.
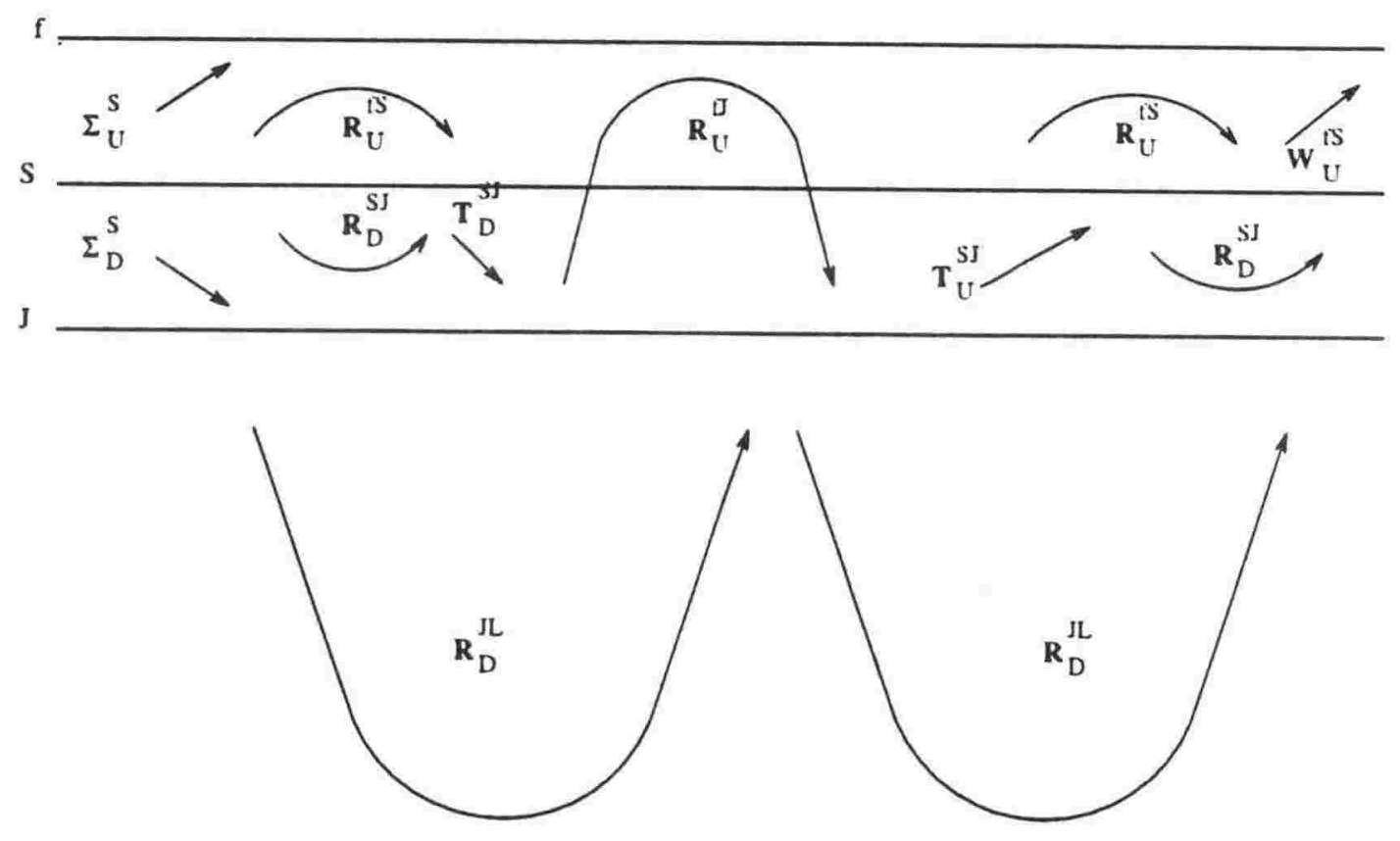

L

Fig. 4.6 Illustration of the separation level $z$ (from Kennett (1988)) 
(iii) Length of time sample, sampling interval and frequency range: The response spectrum contains the information about the whole seismogram relevant to the slowness range and choice of phases considered. If the time interval chosen is too short, then when the inverse Fourier transform is performed, energy arriving after the end of the chosen time span will actually appear at the beginning of the seismogram - a "wraparound effect". This late arriving energy may seriously contaminate the true onset of the waveform and hinder interpretation. This effect may be avoided by using a time series long enough to accomodate all arriving energy groups. An example of the wraparound effect and its elimination are shown in Figure 4.7.

The length of the time series is given by NT, where $\mathrm{N}$ is the number of samples and $\mathrm{T}$ is the sampling interval in seconds. The number of sample points greatly influences the computation time of the Fourier transforms, and therefore it is preferable to increase the sampling interval rather than the number of samples. Care must be taken here, however. The sampling frequency directly controls the frequency content which may be observed in the seismogram. The

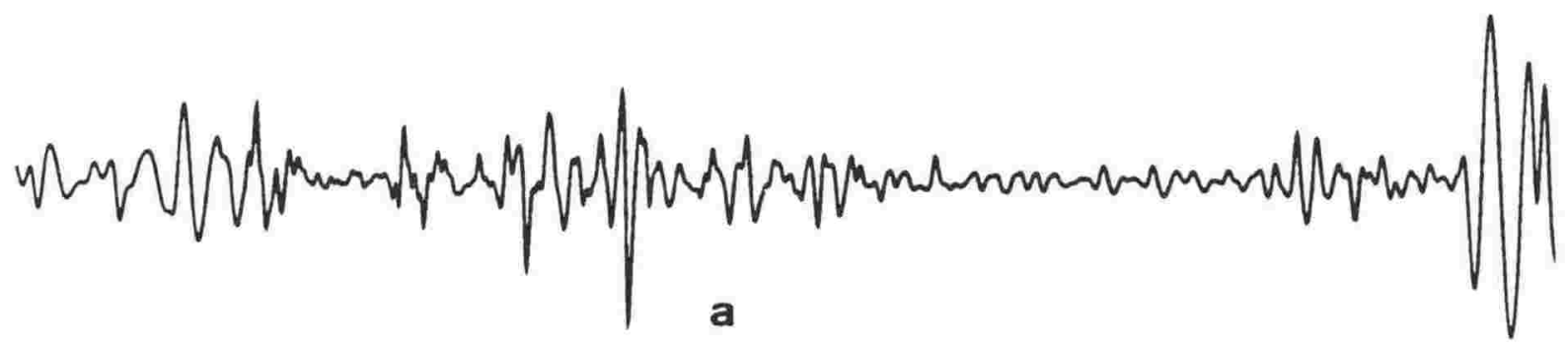

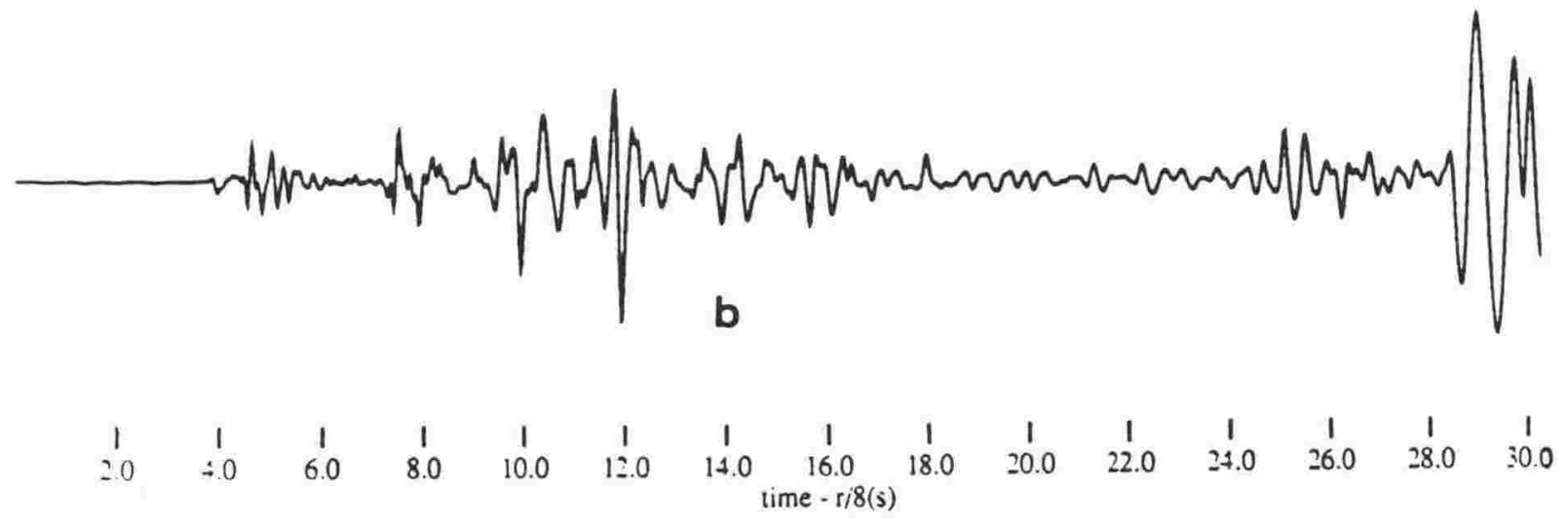

Fig. 4.7 Contamination of the signal onset by "wraparound" 
maximum frequency observable is equal to the Nyquist frequency, that is, the frequency about which the spectrum "folds". Moreover, when using the reflectivity method, it is advised (Kennett, 1983) that the maximum frequency to be modelled should not be much larger than half the Nyquist frequency. This imposes a severe limit on the sampling interval chosen. For example, if one wants to model frequencies as high as $10 \mathrm{~Hz}$, one requires a Nyquist frequency of $20 \mathrm{~Hz}$, and a sampling frequency of $40 \mathrm{~Hz}$, or a sampling interval of $0.025 \mathrm{~s}$. If the phases being modelled arrive at the receiver over a time span of, say, 20 s, then a minimum of 800 points should be used. Since the Fast Fourier Transform algorithm requires the number of samples to be a power of 2 , the best choice would be 1024 points at a sampling interval of $0.025 \mathrm{~s}$. In this study, since the frequency content in the real seismograms was high, and the P-coda extended to about 20s, the best combination for modelling the $\mathrm{P}$-wavetrain was 2048 points at 0.015 sampling interval, i.e a Nyquist frequency of around $33 \mathrm{~Hz}$.

(iv) Slowness range and slowness interval: The integral over horizontal slowness $p$ in equation (4.21) need not, in practice, be carried out from $p=0$ to $p=\infty$. but the range of $p$ may be chosen so that only the phases of interest are included in the chosen time span. This depends on whether one is dealing with $\mathrm{P}$ waves or $\mathrm{S}$ waves or both, on the maximum velocities in the model and on the epicentral distance being considered. For a horizontally plane-layered medium, the slowness $p$ is constant for a ray travelling through the structure, and is given in any layer by

$$
p=\frac{\sin i}{v}
$$

where $i$ is the angle made by the ray with the vertical and $v$ is the wave velocity in that layer. It is thus clear that the range of $p$ can be reduced straightaway to that corresponding to angles of incidence $0-90^{\circ}$. Thus the maximum value of $p$ can safely be taken as $\frac{1}{v_{\min }}$ where $v_{\min }$ is the minimum velocity encountered in the model. The lower limit of $p$, on the other hand, must be small enough to ensure that all head waves are included in the response. In general, then, the lowest value of $p$ must be smaller than $\frac{1}{v_{\max }}$, where $v_{\max }$ is the maximum velocity encountered in the model. If the range of $p$ need be further restricted (for increased efficiency in computing time), then a simple test can be carried out using 2-dimensional ray tracing through the model and observing the minimum and maximum apparent velocities of the main arrivals or the phases of interest, and calculating $p_{\min }$ and $p_{\max }$ accordingly. Such a method is utilised by Fuchs and Muller (1971).

The integral over $p$ is carried out numerically, using a simple trapezoidal rule. Figure 4.8 shows the variation of the real part of the integrand in equation (4.21) as a function of $p$, at a frequency of $6 \mathrm{~Hz}$. It is seen that the integrand is highly oscillatory, and moreover, it can be shown that this oscillatory nature gets more severe at higher frequencies. It is therefore clear that 
if the whole response is to be recovered from this function, the sampling of $p$ must be made very fine. In most applications in this study, the slowness interval was set less than $0.0001 \mathrm{~s} / \mathrm{km}$.

(v) Source mechanism: The response produced at a given point on the surface depends strongly on the radiation pattern produced by the source. The program used here assumes a point moment tensor source at arbitrary depth below the free surface, with the "observation point" at an arbitrary azimuth. Figure 4.9 shows the fault plane geometry and the angles used to completely define the moment tensor, following the axes convention used in Aki and Richards (1980). For pure shear faulting in an isotropic medium, the moment tensor components are given by:

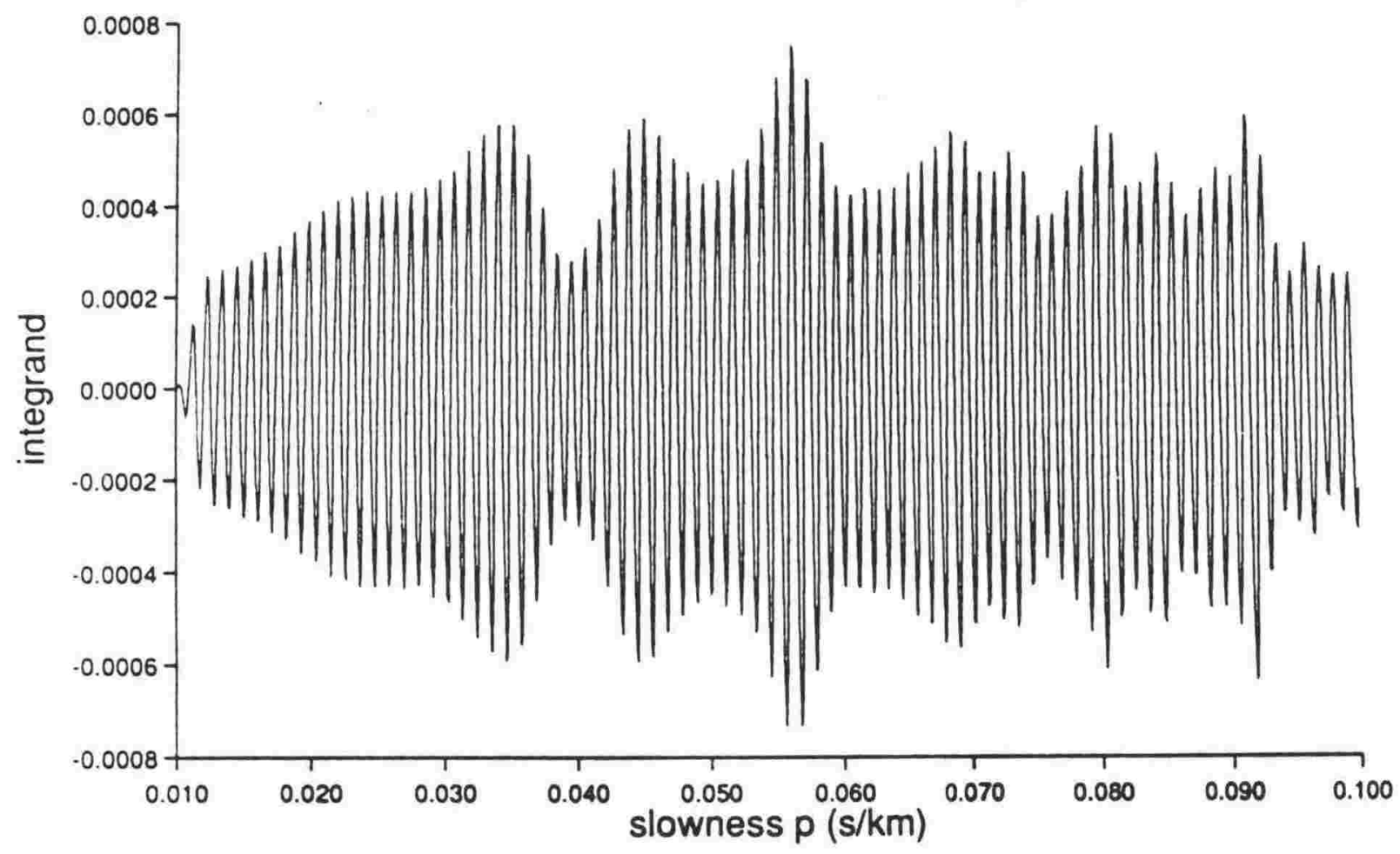

Fig. 4.8 Real part of the integrand in equation 4.21 , at $6 \mathrm{~Hz}$ 


$$
\begin{aligned}
M_{x x}= & -M_{o}\left(\sin \delta \cos \lambda \sin 2 \phi_{s}+\sin 2 \delta \sin \lambda \sin ^{2} \phi_{s}\right) \\
M_{x y}= & M_{y x}=M_{o}\left(\sin \delta \cos \lambda \cos 2 \phi_{s}+(1 / 2) \sin 2 \delta \sin \lambda \sin 2 \phi_{s}\right) \\
M_{x z}= & M_{z x}=-M_{o}\left(\cos \delta \cos \lambda \cos \phi_{s}+\cos 2 \delta \sin \lambda \sin \phi_{s}\right) \\
M_{y y}= & M_{o}\left(\sin \delta \cos \lambda \sin 2 \phi_{s}-\sin 2 \delta \sin \lambda \cos ^{2} \phi_{s}\right) \\
M_{z y}= & M_{y z}=-M_{o}\left(\cos \delta \cos \lambda \sin \phi_{s}-\cos 2 \delta \sin \lambda \cos \phi_{s}\right) \\
M_{z z}= & M_{o} \sin 2 \delta \sin \lambda
\end{aligned}
$$

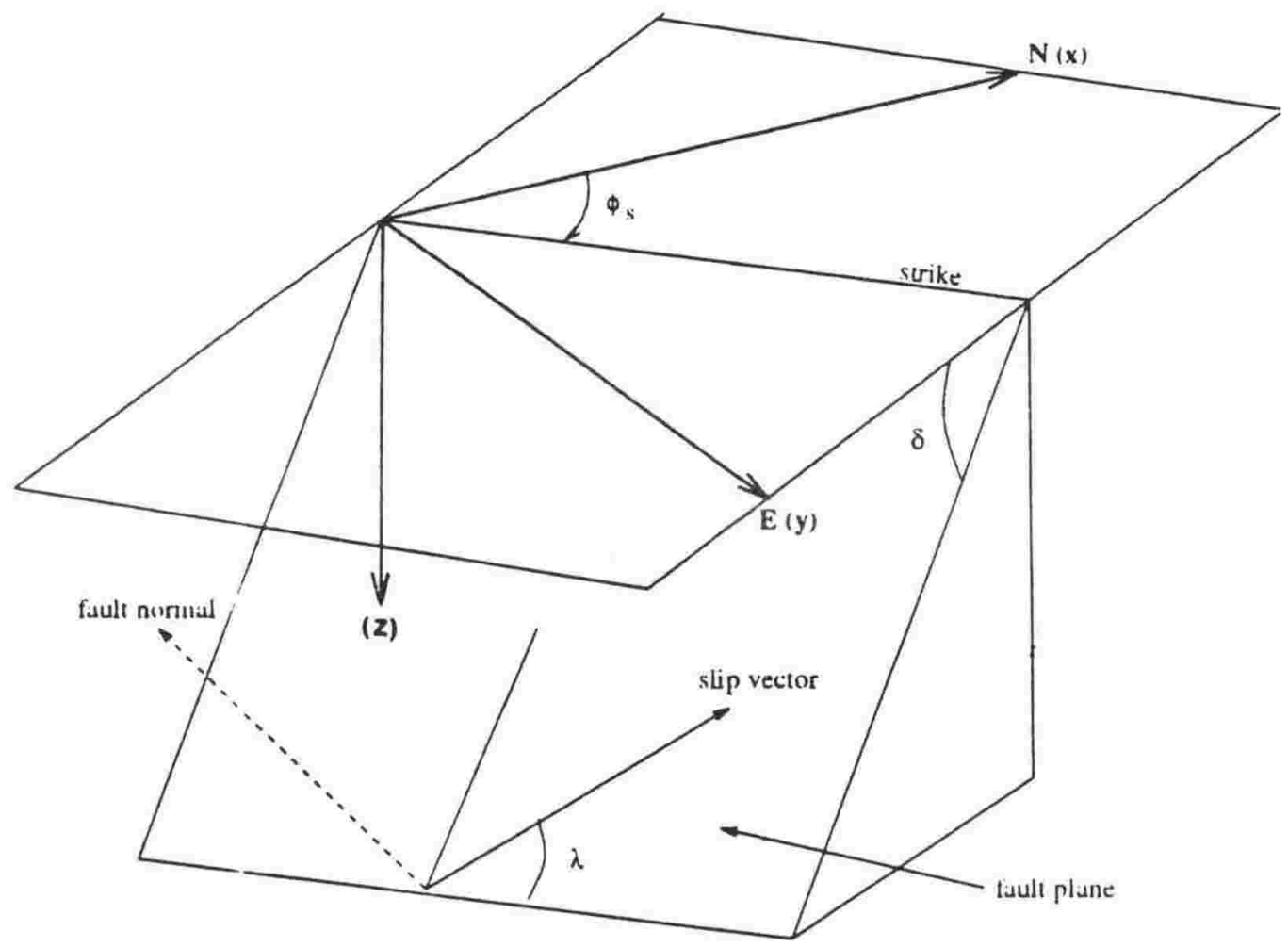

Fig.4.9 An arbitrary fault plane geometry. 
$M_{o}$ is the source seismic moment, defined by

$$
M_{o}=\mu \mathrm{UA}
$$

where $\mu$ is the shear modulus of the medium, $\mathrm{U}$ is the average slip in the plane of the fault (i.e. the magnitude of the slip vector $\mathbf{u}$ ) and $\mathrm{A}$ is the fault area.

The far-field displacement observed at azimuth $\phi$ from North due to the above source mechanism is termed the far-field radiation pattern and is described in Aki and Richards (1980. p.115) 


\section{Chapter 5}

\section{Waveform Modelling}

\subsection{Introduction}

This chapter describes how the Weber data set has been utilised and the general waveform features are used to obtain information and constraints regarding the possible velocity structure within the subducted Pacific plate, as well as within the overlying continental crust. This has been done by means of whole waveform modelling using synthetic seismograms as generated by the reflectivity method of Kennett (1983) discussed in the previous chapter. The aim of such modelling was not to arrive at a detailed and unique velocity model. This would not be possible, for reasons listed below. The approach here has been to

a) identify features of the waveforms which are common to the majority of the seismograms in the data set, and which are inferred to be the result of common path effects;

b) deduce, through the modelling process, the origin of the major energy arrivals within the seismogram;

c) explore parameters within the velocity model which have the greatest influence on the character of the synthetic seismogram and which can be constrained to a greater extent than others through comparison with the characteristics of the observed data.

Throughout this process, previous velocity models and results obtained by other workers about interface depths etc. have been taken into consideration in the starting models. However, the presence of high velocity material inferred from array travel times of the first arrival (Chapter 2) has been assumed, and indeed one of the main parameters investigated has been the depth and vertical extent of such a medium.

\subsection{Difficulties and Assumptions}

The kind and amount of waveform modelling carried out must be regarded as a qualitative attempt at identifying features of the velocity model which produce definite characteristics observable in the data. A more detailed quantification of the velocity structure is not possible because of the limitations inherent in the approach:

i. The synthetic seismogram package used here allows only the use of horizontal, homogeneous 
layers. The true structure in the lower North Island, on the other hand comprises the shallow part of the subduction zone and is therefore essentially a 3-dimensional structure. Fortunately, the wave-path geometry involved in the Weber data set recorded at Wellington is such that the rays travel close to an along-strike path. This will have an influence on the incident azimuth of wavefronts arriving at Wellington, as discussed in Chapters 2 and 3, as well as some effect on travel times when compared with a horizontally layered structure, but it is assumed here that the gross characteristics of the waveforms are not seriously distorted by the dipping layers. Crustal phases that are confined to the layers above the subduction interface will be unaffected by the dipping structures, although reverberatory modes between the free surface and the subduction interface should strictly be treated as propagating through a wedge-like structure. Given that the dip of the slab in this region is not severe, and the path is close to the strike direction, this was again not considered. Some attempts have been made to extend the reflectivity method to the case of a dipping "layer package" (e.g. Sandmeier and Wenzel, 1988) but this has not been generalized enough to be as useful for the present application as the method of Kennett. Langston (1977), using a generalized ray theoretical method, computes the teleseismic response of a dislocation source in a dipping layer structure. His results show that an interface dip of up to $10^{\circ}$ does not significantly distort the vertical component $\mathrm{P}$ wave, and the effect is particularly insignificant in the case of a thrust type dislocation source, as is the case with the events of the Weber data set.

ii. The waveforms being modelled in this study originate from shallow earthquakes at regional distances and contain high frequencies. This makes the seismograms complex and hard to interpret and model in any great detail. The response at high frequencies of the medium along the propagation path is such that a high degree of scattering takes place by small-scale heterogeneities, so that no two waveforms, even if originating from the same source region and from events of similar focal mechanisms, will be alike. The effect can also be seen in the fact that correlation of the high frequency signal for any one event recorded across the L-network is difficult.

Moreover, although the source region for the whole set of earthquakes is relatively small compared with the epicentral distance, the difference in this distance, as well as in the depth, between individual events means that corresponding phases arrive at slightly different times at Wellington, and this makes it difficult to correlate individual phases between one event and another with any certainty. For example, the maximum range in epicentral distance is about 20 $\mathrm{km}$, which gives a variation of the order of $2.5 \mathrm{~s}$ for $\mathrm{S}$ - P time differences.

In view of all this, it is unreasonable to attempt to refine the velocity model to an extent which will model individual peaks in a seismogram for this data set.

iii. It has to be assumed that the velocity structure between source and receiver is uniform, 
whereas it is likely there will be some heterogeneity or gradual change in structure on going from Hawkes Bay to Wellington, especially in the shallower layers.

iv. The effect on the waveforms of seismic velocity anisotropy has not been taken into consideration.

Modelling has been concentrated on the vertical component seismogram and on the Pwavetrain, although the S-wavetrain is also included in many of the synthetic seismograms.

\subsection{Common Features of the Observed Waveforms}

The available data represent wavetrains from a large number of sources in a relatively small source region having approximately the same focal mechanism, and traversing much the same wayepath. Therefore, in spite of the difficulties listed above, we would expect to find reproducible waveform features throughout the data set that can be attributed to source and general wavepath effects and which could therefore be modelled by suitable earth structures. Such features have already been described to an extent in Chapter 2. More data are presented here.

Figure 5.1 illustrates a number of seismograms from the Weber data set recorded at stations of the L-network. As far as possible, the records from the same station (KSE) are displayed, so that differences in waveform are not confused with differences in site response. The diagram is intended to emphasize the common waveform features that were modelled, and at the same time to show the extent of differences between any two seismograms. Only vertical component seismograms are shown.

As was illustrated in Chapter 2, the arrival of a second phase approximately 4 seconds after the initial P onset appears to be a stable feature of the data, as does the difference in frequency content between this phase and the first 4 seconds, this difference being observable visually in most cases. The largest amplitudes within the $\mathrm{P}$ wavetrain generally occur within the lower frequency second phase. On looking at a record section of the data across the L-network, some individual sharp peaks within this phase can be unambiguously traced across the array (Figure 5.2). One such phase generally occurs at around 4 - 5 s after the initial onset, whereas a second one occurs at around $7-9 \mathrm{~s}$. Where these phases travel undistorted across the array, it was possible to pick the arrival times at their peaks sufficiently accurately to estimate their phase velocity in the same way as was done for the first pulse. The apparent velocities obtained in this way were in the range $6.0-6.6 \mathrm{~km} / \mathrm{s}$. It would be reasonable to expect the waveform modelling to reproduce such phases and provide an interpretation. 
In the classical definition of seismic phases, the terms $P^{*}$ and $P_{g}$ are used to describe prominent arrivals shortly after $P_{n}$, and are interpreted as crustal phases generated by a shallow earthquake, travelling in the "lower" and "upper" crust at velocities of approximately 6.5 and 6.0 $\mathrm{km} / \mathrm{s}$ respectively (Aki and Richards, 1980) However, the term $\boldsymbol{P}_{\boldsymbol{g}}$ has also been widely used in a more general sense in the literature for the prominent and long-wavetrain phase arriving after $P_{n}$, from crustal earthquakes at distances greater than about $100 \mathrm{~km}$, and travelling at around $6 \mathrm{~km} / \mathrm{s}$. This phase is usually interpreted as a group of multiply reflected P-SV waves propagating in a crustal waveguide bounded by the Moho and the free surface, or within some other layers of the crust ( e.g. Langston and Helmberger, 1974; Barker, Der and Mrazek, 1981; Langston, 1982; Banda et al, 1982; Olsen, Braille and Stewart, 1983; Pederson and Campillo, 1991; Campillo and Paul, 1992). The equivalent of $P_{g}$ that follows the first $\mathrm{S}$ arrival is termed $L_{g}$. The $P_{g}$ phase is sometimes also called $\bar{P}$ (Olsen, Braille and Stewart, 1983). It shall be shown later in this chapter that the nature of the dominant second arrival in the observed data conforms to the latter interpretation of $P_{g}(\bar{P})$, and for convenience we shall henceforth choose to call this arrival $\bar{P}$.

Figure 5.3 is a closer look at some seismograms which had a particularly clear first onset, due partly to a low signal-to-noise ratio at the start of the signal, and which illustrate the nature of the first pulse common to many of the records. Further examples of this phase, traced across the L-network, have been shown in Figure 2.6. This first arrival can be unambiguously referred to as $P_{n}$. The $P_{n}$ wavelet is simple and of lower frequency than the directly-following complex phase. The shape of the first onset, and its relationship with the following phases is another feature which will be modelled in the synthetic seismograms and which will provide some constraints on the nature of the medium through which the phase propagates.

Whereas the nature of the first arrival can be assigned to a critically refracted wave, $P_{n}$, it is more difficult to assign a label to the immediately-following high-frequency signal. Similar features have sometimes been referred to as an extended $P_{n}$ waveform (e.g. Langston, 1982) although it will be shown that, in this case, it does not necessarily consist only of refracted and turning waves. For the sake of easy reference, it will be referred to here as $\mathrm{P}_{\mathrm{hf}}$.

An attempt was also made at enhancing some features contained in the high frequency phase by stacking the traces for a given event over all the stations of the L-network. This technique was, however, abandoned as it did not yield useful information, mainly due to the rapid breakdown of coherence in the signal following $P_{n}$.

It is proposed that this phase of the seismogram can be regarded as composed of multiple arrivals (reflections/refractions), originating at some level close to the high velocity medium that gives rise to $P_{n}$. Such features will be the subject of one part of the waveform modelling process. 

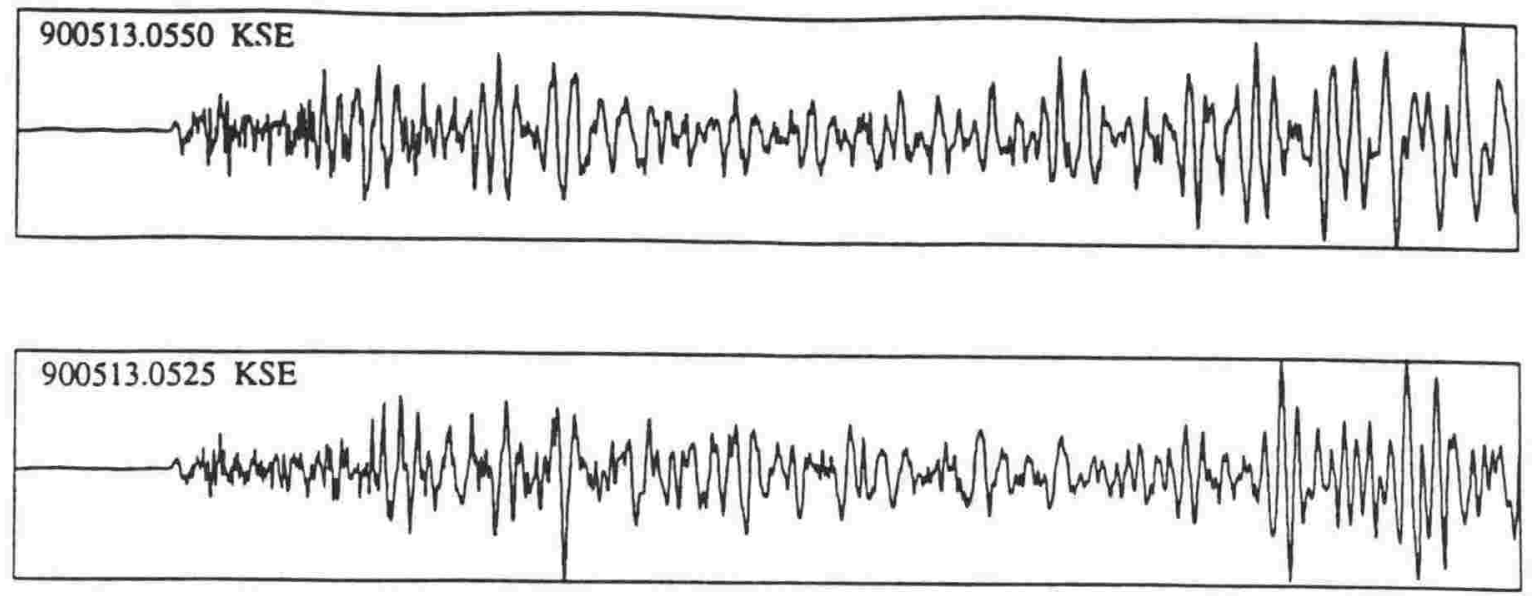

$900513.0802 \mathrm{KSE}$
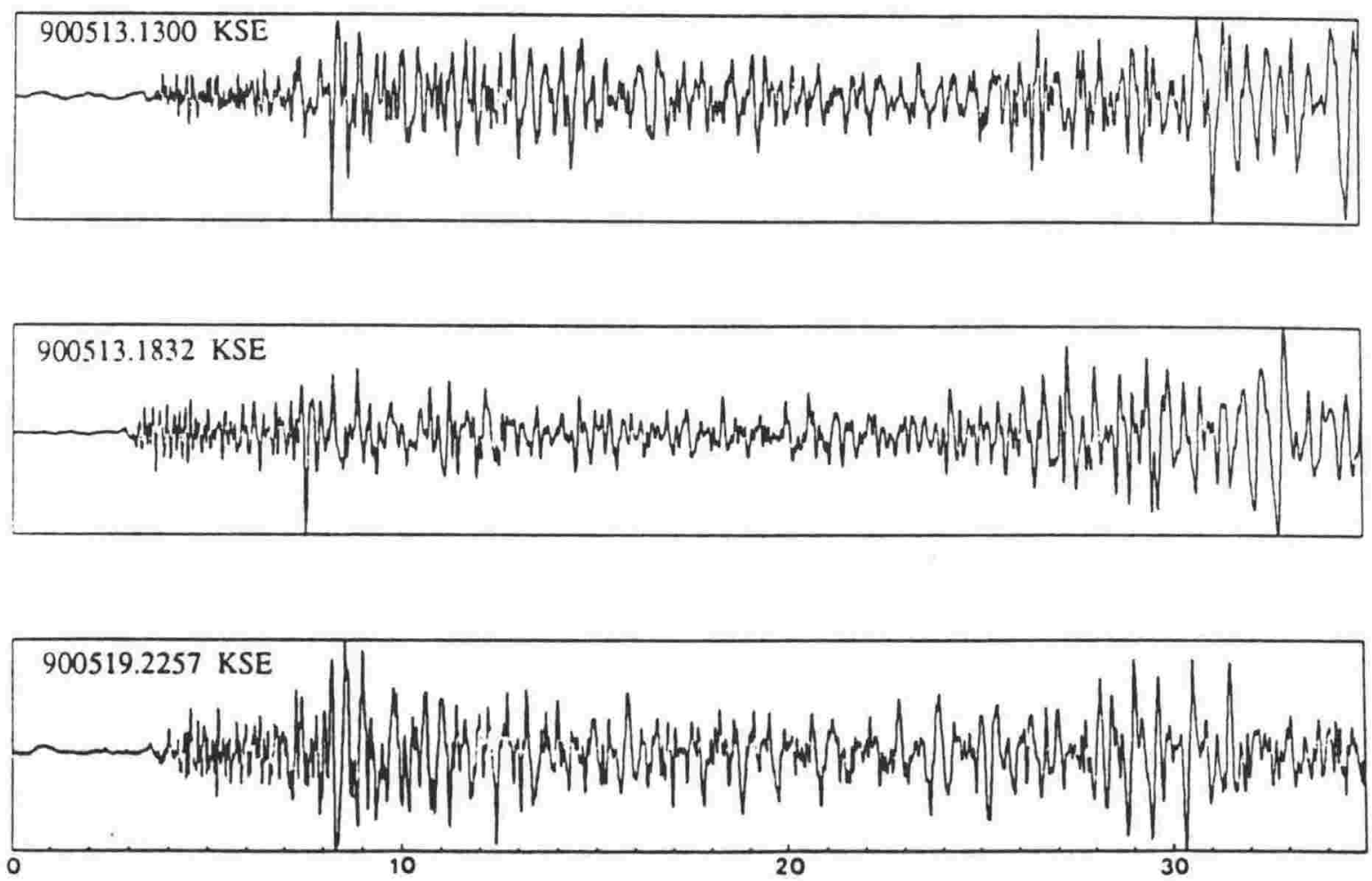

Fig. 5. 1 Some examples of vertical component seismograms from the Weber data set recorded on L-network stations at Wellington. These examples illustrase some of the common waveform features modelled by synthetic seismograms. 

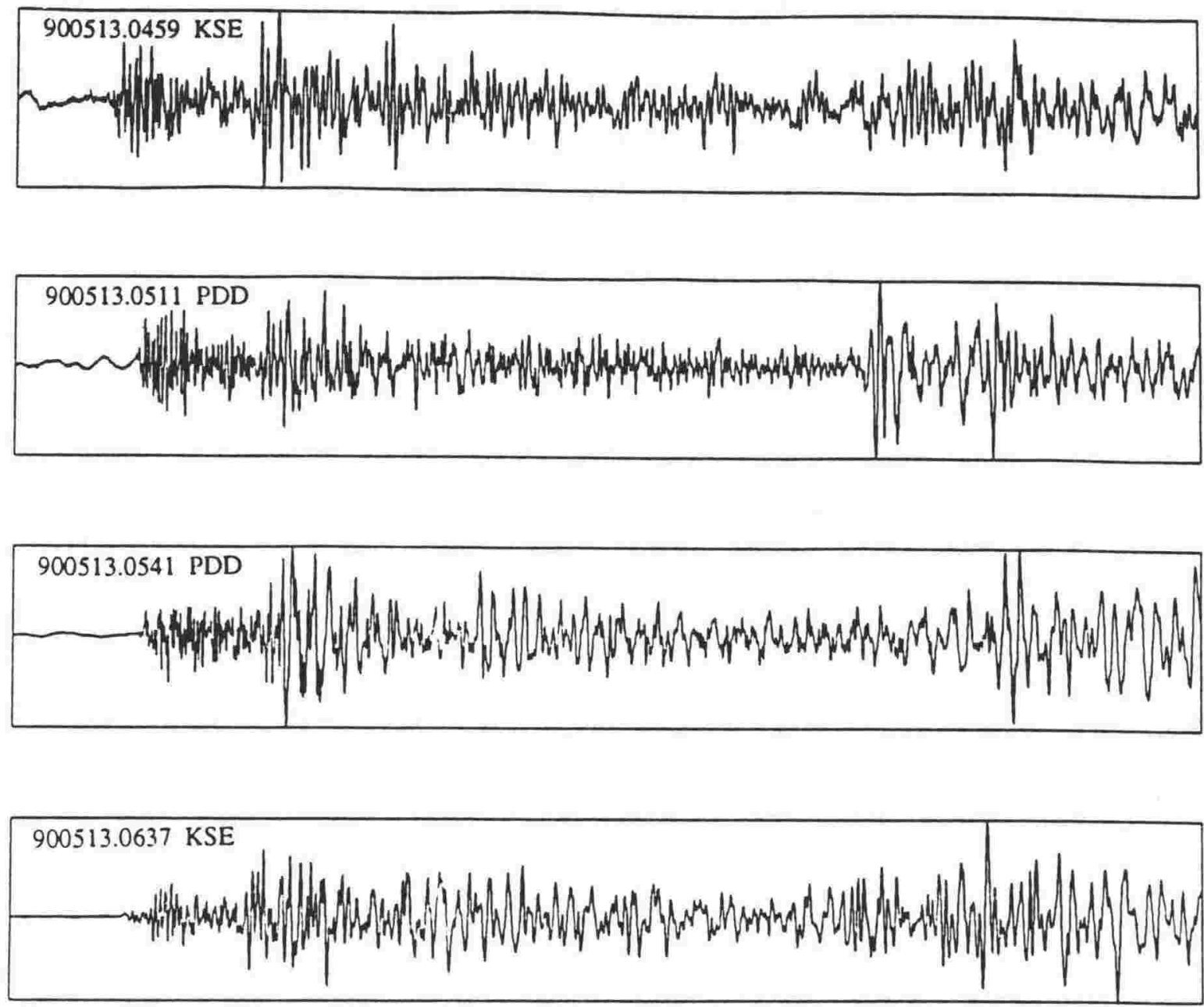

900513.0640 KSE

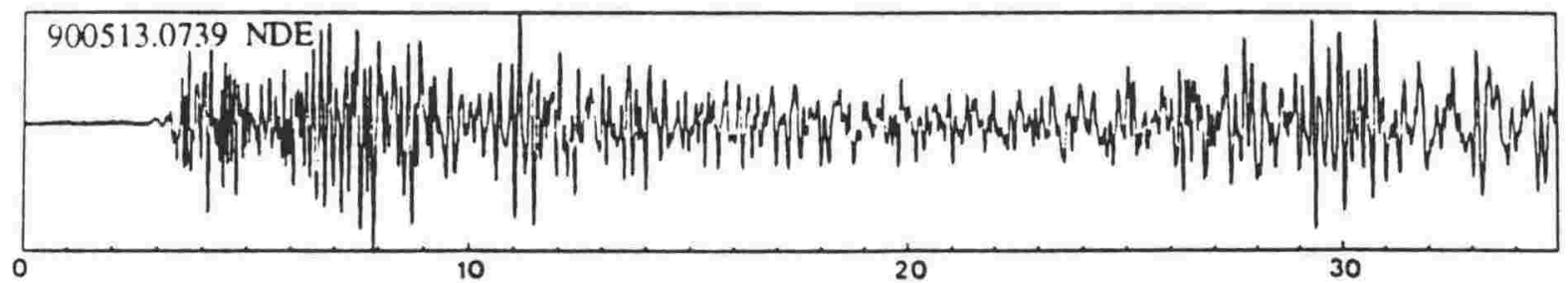

Fig. 5.1 (cont.) 

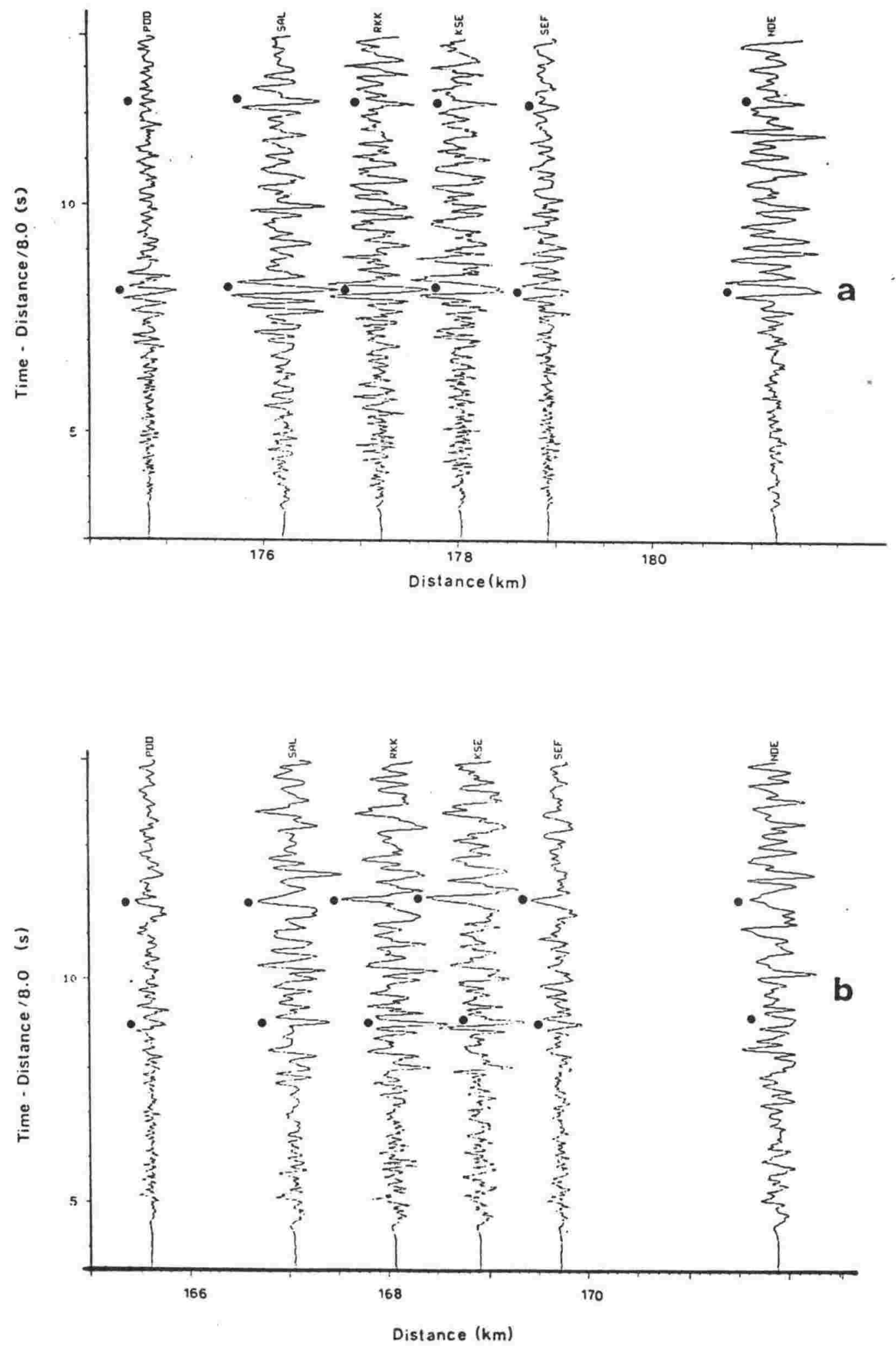

Fig. 5.2 Two examples of record sections across the L-network showing the correlation of some individual phases (marked by dots) within the $\bar{P}$ group a) Event 900513.0559 b) Event 900513.0550. 

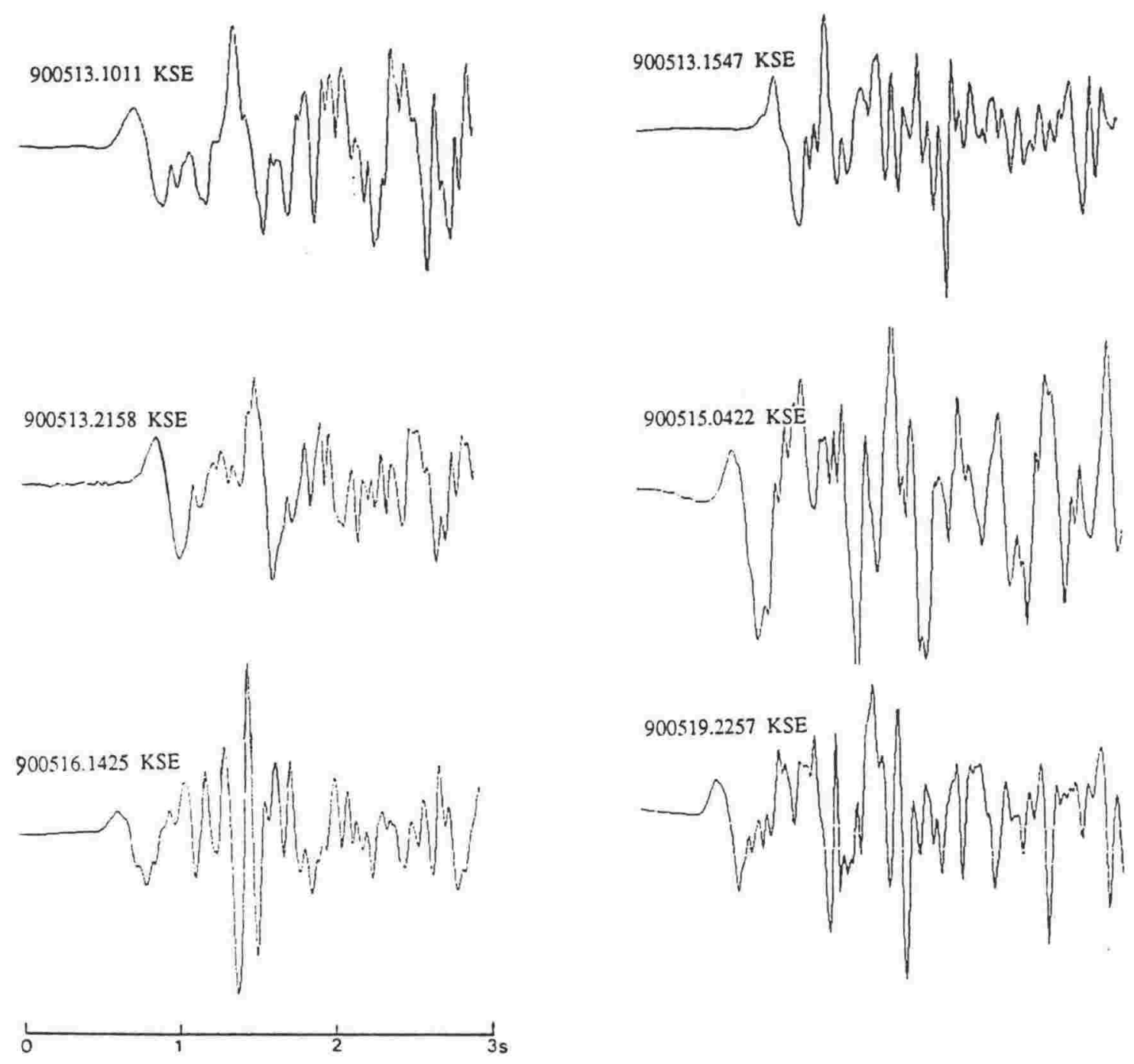

Fig. 5.3 Examples of vertical component seismograms from the Weber data set, showing the similarity of pulse shape for the first $\left(\mathrm{P}_{\mathrm{n}}\right)$ arrival.

\subsection{Basic Velocity/Attenuation Model}

The starting velocity/attenuation model, as well as the parameters used for the computation of the synthetic seismograms (Section 5.5) were arrived at after a lengthy process of adjustment until the synthetic data closely resembled the "average seismogram" from the Weber data set. The gross features of the velocity model were largely based on previous velocity and structural models obtained by other workers in the Hawkes Bay and Wellington areas. The model has been labelled MOD01 and is shown in Table 5.1. A $V_{P} / V_{S}$ ratio of 1.75 in all layers was assumed. The density in each layer was calculated from the Nafe-Drake relationship 


$$
\rho=0.252+0.379 v_{P}
$$

as suggested by Fuchs and Muller (1971).

Figure 5.4 shows the P-velocity profile compared with previous models of the lithospheric structure. Following the results of Chapter 3, a P-velocity of $8.75 \mathrm{~km} / \mathrm{s}$ was assumed to exist in the lowermost layer, below a "normal" $8.0 \mathrm{~km} / \mathrm{s}$ uppermost mantle layer. In the basic model, this high-velocity medium has been represented as a half-space, however one aspect of the modelling considers the effects of a layer of this medium of varying thickness and velocity, as shall be discussed later in this chapter.

The $\mathrm{P}$ and $\mathrm{S}$ attenuation parameters, $\mathrm{Q}_{\mathrm{P}}^{-1}$ and $\mathrm{Q}_{\mathrm{S}}^{-1}$ were assumed to be independent of frequency. The values shown in Table 5.1 are ones which give a reasonable reproduction of the relative amplitudes and frequency content of the various portions of the seismograms, however their effect on the synthetic seismograms will be discussed in a later section of this chapter.

The separation level discussed in Chapter 4, which allows some degree of differentiation between the responses of the media above and below it, was taken to coincide with the subduction interface, shown in Figure 5.5 at $18 \mathrm{~km}$ depth. Thus in most of the modelling of the Weber data set, the source was embedded in the layers above the separation interface, and it was thus easier to visualize the total response as having a "deep" contribution and a "crustal" contribution.

The source-time function was taken to be a delta function at time $t=0$. For a simple doublecouple source, the far-field displacement is in effect proportional to the time derivative of the source-time function, which is proportional to the delta function, and the far-field spectrum is then approximately a constant (Bullen and Bolt, 1985, p.418). This assumption was probably justified for the aftershocks (magnitude < 4.5), but not for the mainshock (magnitude 6.3 ) which would exhibit more complex source effects. The waveform for the mainshock was in fact not

Table5. 1 Starting velocity model MOD01 used in waveform modelling

\begin{tabular}{|cccccc|}
\hline $\begin{array}{c}\text { Depth to top } \\
\text { of layer }(\mathrm{km})\end{array}$ & $\begin{array}{c}\text { P-velocity } \\
(\mathrm{km} / \mathrm{s})\end{array}$ & $\begin{array}{c}\text { S-velocity } \\
(\mathrm{km} / \mathrm{s})\end{array}$ & $\begin{array}{c}\text { Density } \\
(\mathrm{g} / \mathrm{cm})\end{array}$ & $\mathrm{Q}_{\mathrm{p}}^{-1}$ & $\mathrm{Q}_{\mathrm{S}}^{-1}$ \\
\hline & & & & & \\
0.0 & 4.00 & 2.28 & 2.20 & 0.010 & 0.02 \\
1.5 & 5.50 & 3.14 & 2.30 & 0.002 & 0.004 \\
10.0 & 6.20 & 3.54 & 2.60 & 0.002 & 0.004 \\
18.0 & 6.50 & 3.71 & 2.75 & 0.002 & 0.004 \\
25.0 & 7.60 & 4.34 & 3.06 & 0.001 & 0.002 \\
30.0 & 8.00 & 4.57 & 3.28 & 0.001 & 0.002 \\
36.0 & 8.75 & 5.00 & 3.58 & 0.001 & 0.002 \\
\hline
\end{tabular}




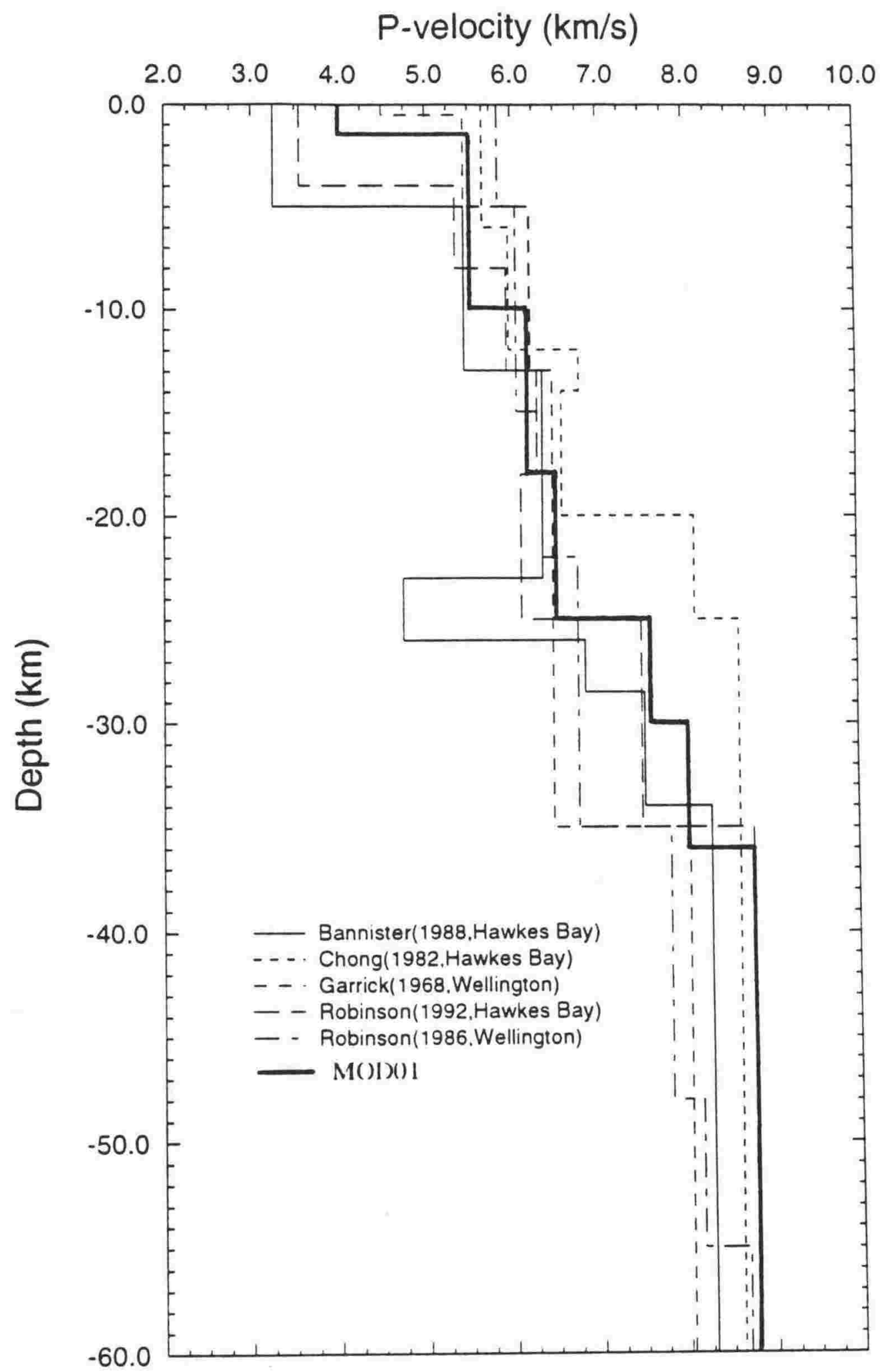

Fig. 5.4P-velocity profiles for the Hawkes Bay and Wellington regions obtained by previous workers as explained in the legend, together with the profile corresponding to the basic model MODOI. 
included when looking for common features to model.

For the purposes of waveform modelling, the earthquake source was placed at $12 \mathrm{~km}$ depth. This lies within the depth range $(5-18 \mathrm{~km})$ arrived at by Robinson (1994) for the Weber aftershocks. The effect of the depth parameter is later investigated (Section 5.8). The dislocation source has a moment tensor representation which may be easily varied during the modelling to simulate any required fault plane geometry. For the main Weber data set, enough work had been done on the aftershock sequence (see Chapter 2, Section 2.3.1) to constrain the average focal mechanism of the shallow events. The coordinate system for setting up the moment tensor components was as given in Aki and Richards (1980, p.114). For simplicity, the North axis was taken to coincide with the strike of the subducted slab (see Figure 4.9). After Robinson's work, the appropriate angles for a typical source orientation were chosen to be

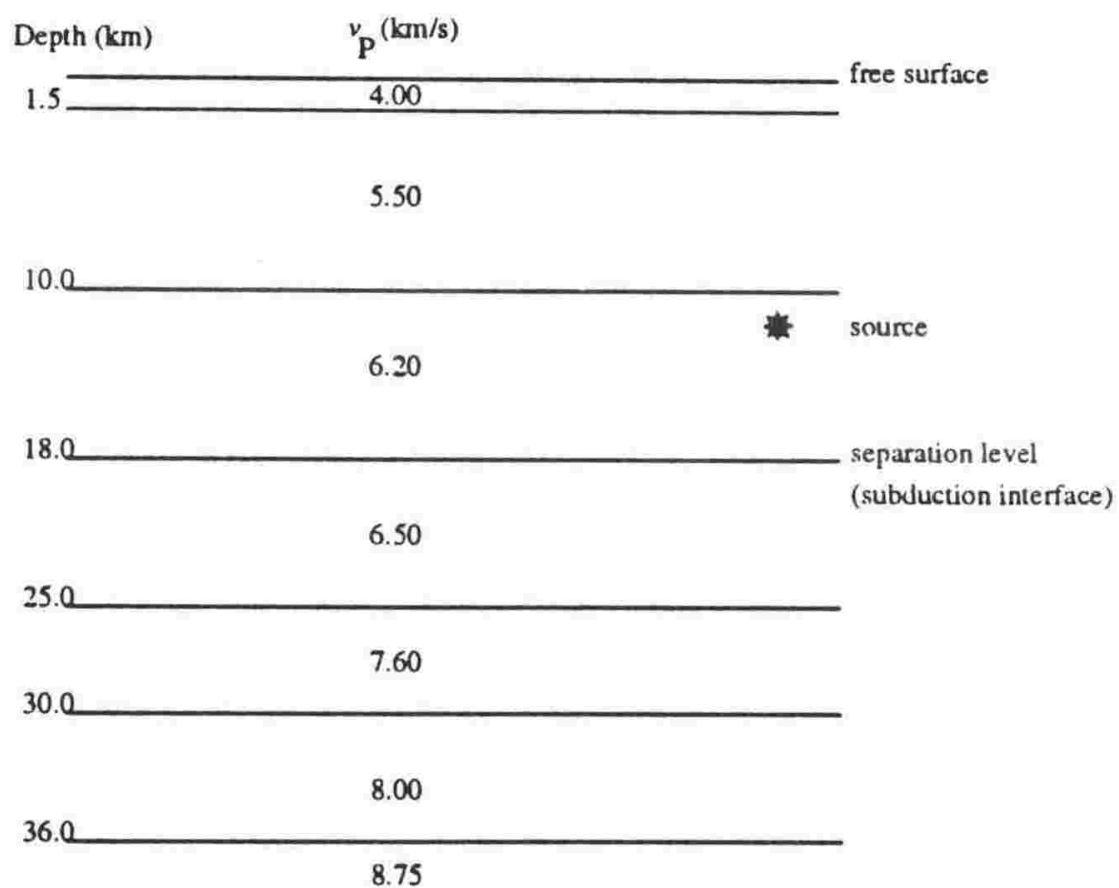

Fig. 5.5 Layer version of MOD01. Source position is indicated by a star. 


$$
\phi_{f}=170^{\circ} ; \delta=35^{\circ} ; \lambda=110^{\circ} ; \phi=190^{\circ}
$$

where $\phi_{f}$ is the strike of the fault plane, $\delta$ is its dip, $\lambda$ is the rake (introducing a small component of strike slip) and $\phi$ is the observation azimuth measured clockwise from North. Using the angles $\phi_{f}, \lambda$ and $\phi$, and the formulas in Aki and Richards (1980, p.117), the Cartesian components of the moment tensor are:

$$
\left(\begin{array}{ccc}
-0.094 & -0.335 & -0.332 \\
-0.335 & -0.789 & -0.268 \\
-0.332 & -0.268 & 0.883
\end{array}\right)
$$

It was not intended to model absolute amplitudes of ground motion, and therefore the moment magnitude was taken as unity, and the resulting seismograms were then normalized as necessary.

The final synthetic surface response at the receiver was also convolved with a typical EARSS instrument response so that a more meaningful comparison with the observed seismograms could be made. This was done by multiplying the synthetic spectrum in the frequency domain by the EARSS frequency response function (Chadwick, 1991). This function is described in Appendix D.

The reflectivity program used here only allows homogeneous layers of constant velocity. Velocity gradients must be approximated by a "cascade" of thin layers of increasing or decreasing velocity. In this application, the effect of a continuous velocity change was achieved by making the layers in the cascade thinner than half the shortest wavelength in the spectrum and by ensuring that velocity jumps at the boundaries did not exceed $0.05 \mathrm{~km} / \mathrm{s}$. This often entailed the introduction of a large number of layers, which made the computation of the response very time consuming. For this reason, the presence of velocity gradients in the model was restricted to a few cases where their effect was to be specifically investigated.

\subsection{Features and Explanation of the Synthetic Seismogram}

Unless otherwise stated, the following parameters have been used throughout the computation of the synthetic seismograms that follow in this chapter:

$\begin{array}{ll}\text { Range: } & 175 \mathrm{~km} \\ \text { Frequency: } & 1.0-9.0 \mathrm{~Hz} \\ \text { Sampling interval: } & 0.02 \mathrm{~s} \\ \text { Sample size: } & 2048 \text { points } \\ \text { Slowness range: } & 0.1-0.29 \mathrm{~s} / \mathrm{km}\end{array}$


Reducing velocity: $\quad 8.0 \mathrm{~km} / \mathrm{s}$

Amplitude scaling: True relative amplitudes between displayed waveforms

\subsubsection{Appearance}

Figure 5.6a shows the 3-component synthetic seismogram generated by the velocity/attenuation model of Table 5.1. It is a "full" response, including $\mathrm{P}$ and $\mathrm{S}$ modes at all stages of the propagation, full reverberation in individual layers and free surface reflections.

It is seen that the vertical component seismogram reproduces in a broad sense the characteristics of the data as shown in Figures 5.1 - 5.3, namely a first phase of close-spaced arrivals ( $P_{h f}$ ) preceeded by a single $\mathrm{P}_{\mathrm{n}}$ pulse, and followed approximately $4 \mathrm{~s}$ later by a larger amplitude, somewhat lower frequency group, which decays rapidly into the P-coda. A corresponding higher frequency $S_{n}$ arrival, followed by a large-amplitude " $L_{g}$ " is also clearly visible. The absence of any signal on the transverse component before the $S$ arrival is to be expected in the case of the flat-layered model due to the absence of any SH-wave generation.

It should be noted that, as discussed in Chapter 2, the actual frequency content of the real data is often much higher than $9 \mathrm{~Hz}$, particularly in the $\mathrm{P}_{\mathrm{hf}}$ phase. However, the computation at high frequencies, say up to $15 \mathrm{~Hz}$, becomes impractically time consuming when carried out a large number of times, as was done here. Also, the scattering effect at high frequencies makes it irrelevant to compare in detail a synthetic waveform with the real data. Hence the routine synthetic seismogram generation was carried out at frequencies up to $9 \mathrm{~Hz}$. Figure $5.6 \mathrm{~b}$ shows the same seismogram as in Figure 5.6a (vertical component), computed in the frequency range $3-15$ $\mathrm{Hz}$. The synthetic seismograms at these high frequencies probably suffers from too small a sampling interval in the slowness domain, although the general features of the seismogram are retained.

Figure 5.7 is a long-range profile of the seismograms generated by the same model as in Figure 5.6, and gives a better picture of the speed at which the various phases travel. The section is plotted with a reducing velocity of $8.0 \mathrm{~km} / \mathrm{s}$. The $P_{h f}$ phase appears to move out at approximately $8.0 \mathrm{~km} / \mathrm{s}$, while the $\bar{P}$ group moves at a crustal-type velocity of around $6.0 \mathrm{~km} / \mathrm{s}$. In this figure, the relative amplitudes between successive traces are not true, but the trace amplitudes have been normalized to the same maximum.

Figure 5.8a shows, on the same time scale, three sample vertical-component seismograms from the real data, together with the vertical component synthetic seismogram, for comparison, and emphasises the similarity in appearance. The reduced $P_{n}$ travel time of $4 s$ on the synthetic data corresponds at this distance to a travel time of $25.9 \mathrm{~s}$, comparing well with the average observed travel time of around $26.5 \mathrm{~s}$ for the real data.

Figure $5.8 \mathrm{~b}$ shows the first 4 seconds of the synthetic seismogram compared with those of a 


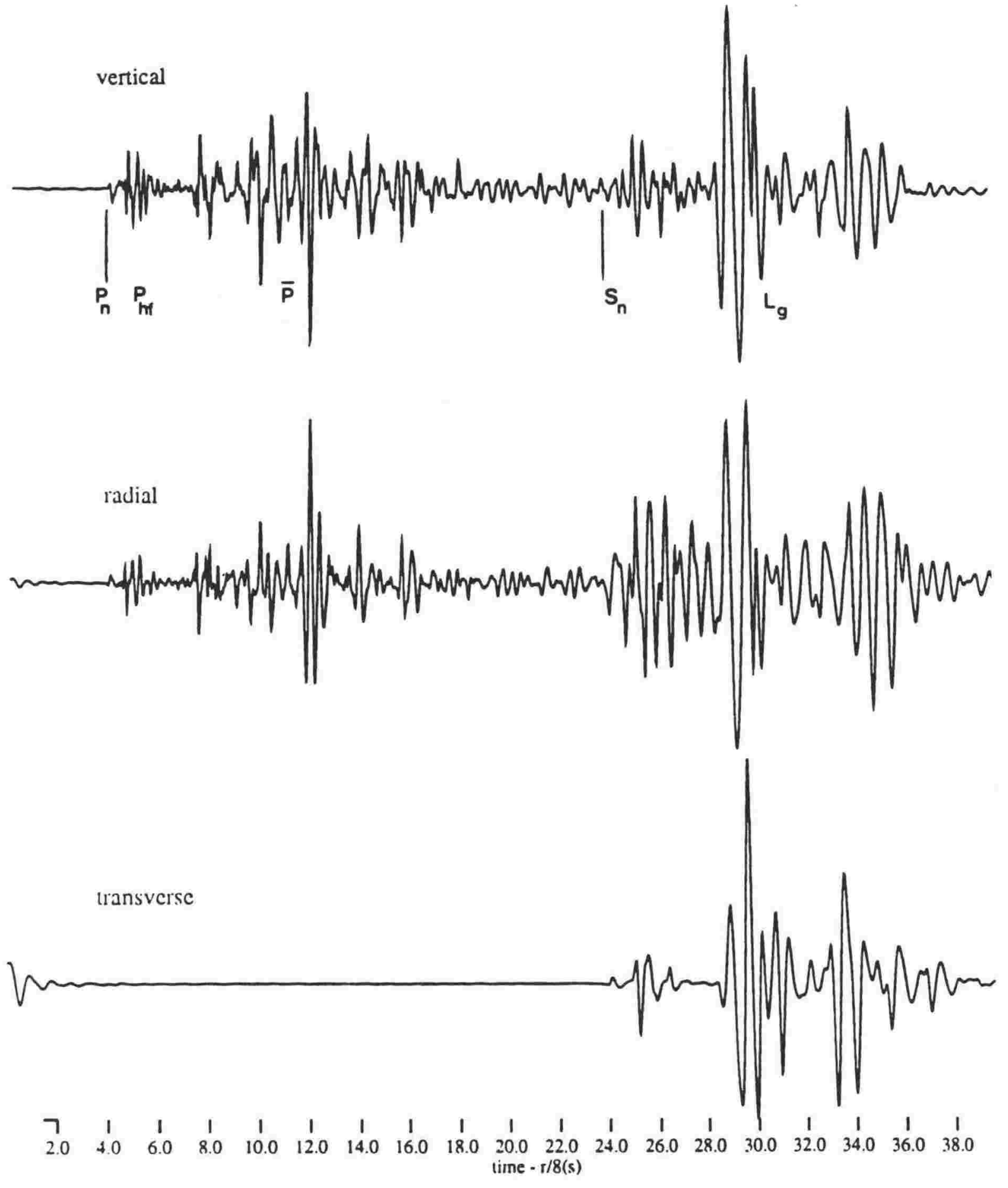

Fig. 5.6a 3-component synthetic seismogram generated by the basic model MODOI. 

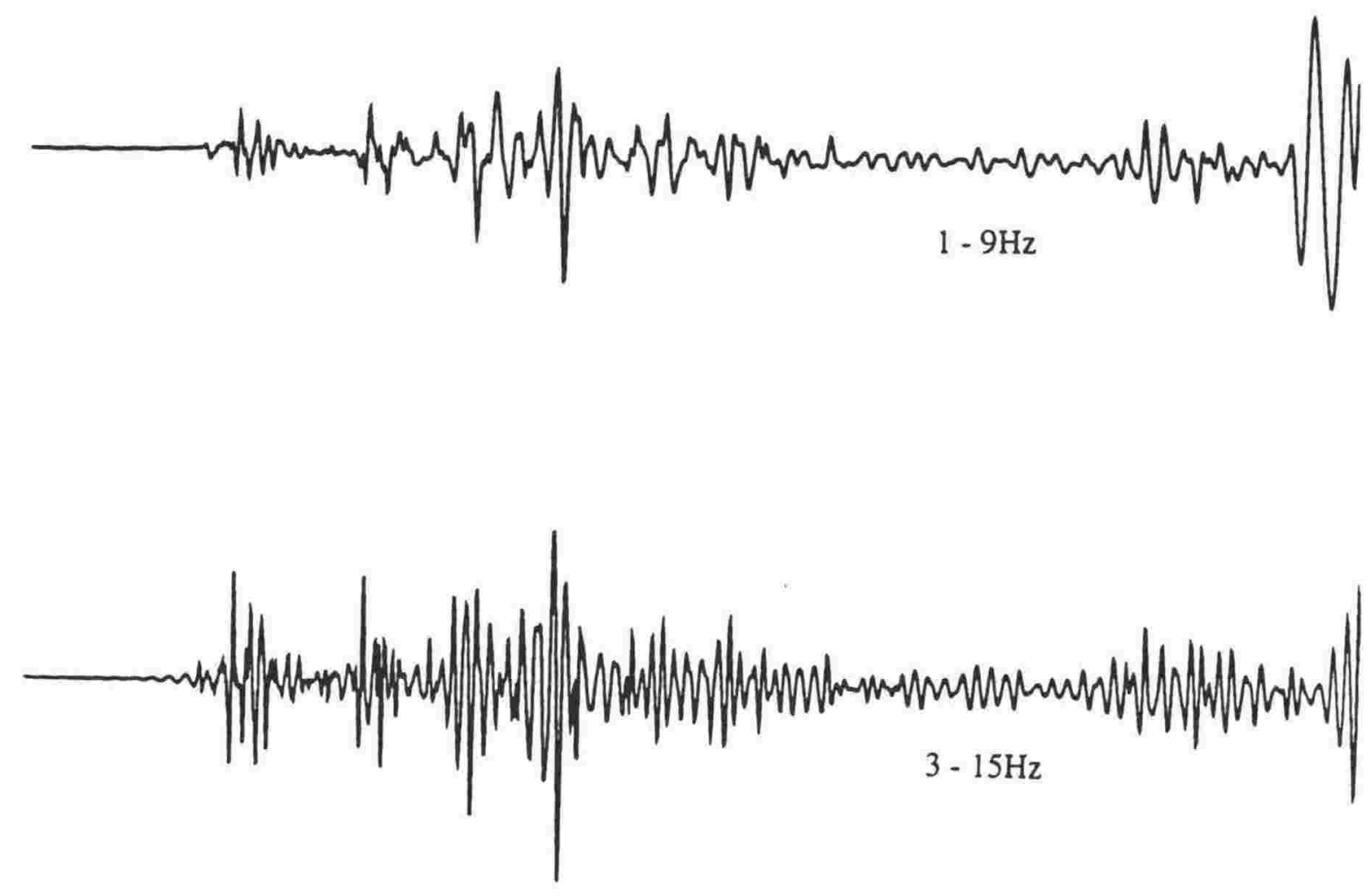

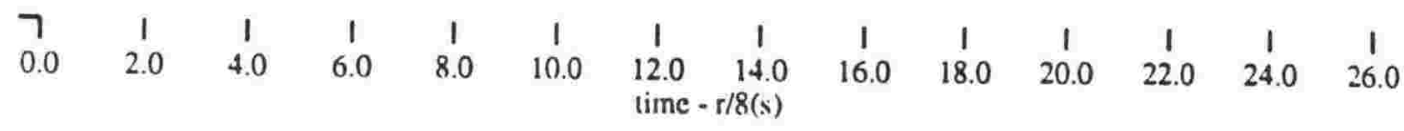

Fig. 5. 6b Vertical component of same seismogram as Figure 5.6a, computed in the frequency range $3.15 \mathrm{~Hz}$

real seismogram, and illustrates the similarity of the $\mathrm{P}_{\mathrm{n}}$ pulse and its relationship with the $P_{h f}$ phase.

This model therefore gives a reasonable simulation of the observed data, and it will now be appropriate to try to identify, in as much detail as possible, the origin and nature of the various parts of the synthetic seismogram, and to attribute these same features and their interpretation to the real data. Having achieved this, the next stage in the modelling is to consider various sections of the velocity profile and source and attenuation parameters in turn, and observe the way in which changes in these parameters will affect the appearance of the corresponding parts of the synthetic seismogram. In this way, some constraints and conclusions can hopefully be made on certain features of the profile.

But before that, we have to be certain that the synthetic seismogram itself is understood and interpreted in as much detail as possible, in other words, that the various phases and groups of phases on the seismogram are identified. There are two ways in which this can be done. The first is by matching travel times using ray-tracing. The second makes use of the "wavefield 


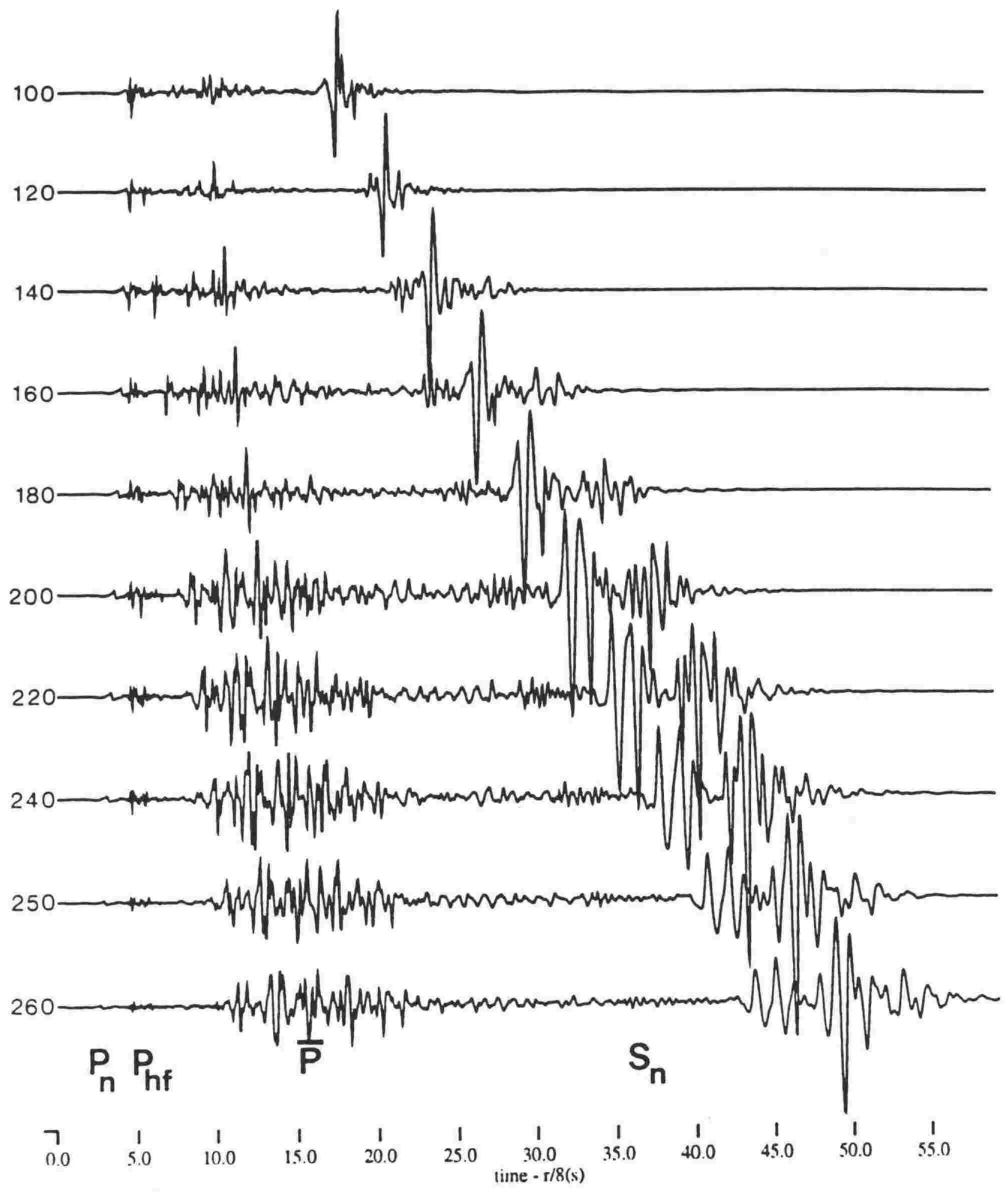

Fig. 5.7 Long-range profile for the same synthetic seismogram as in Figure 5.6. Reducing velocity is 8.0 $\mathrm{km} / \mathrm{s}$. Distances are shown in $\mathrm{km}$ on the left. 


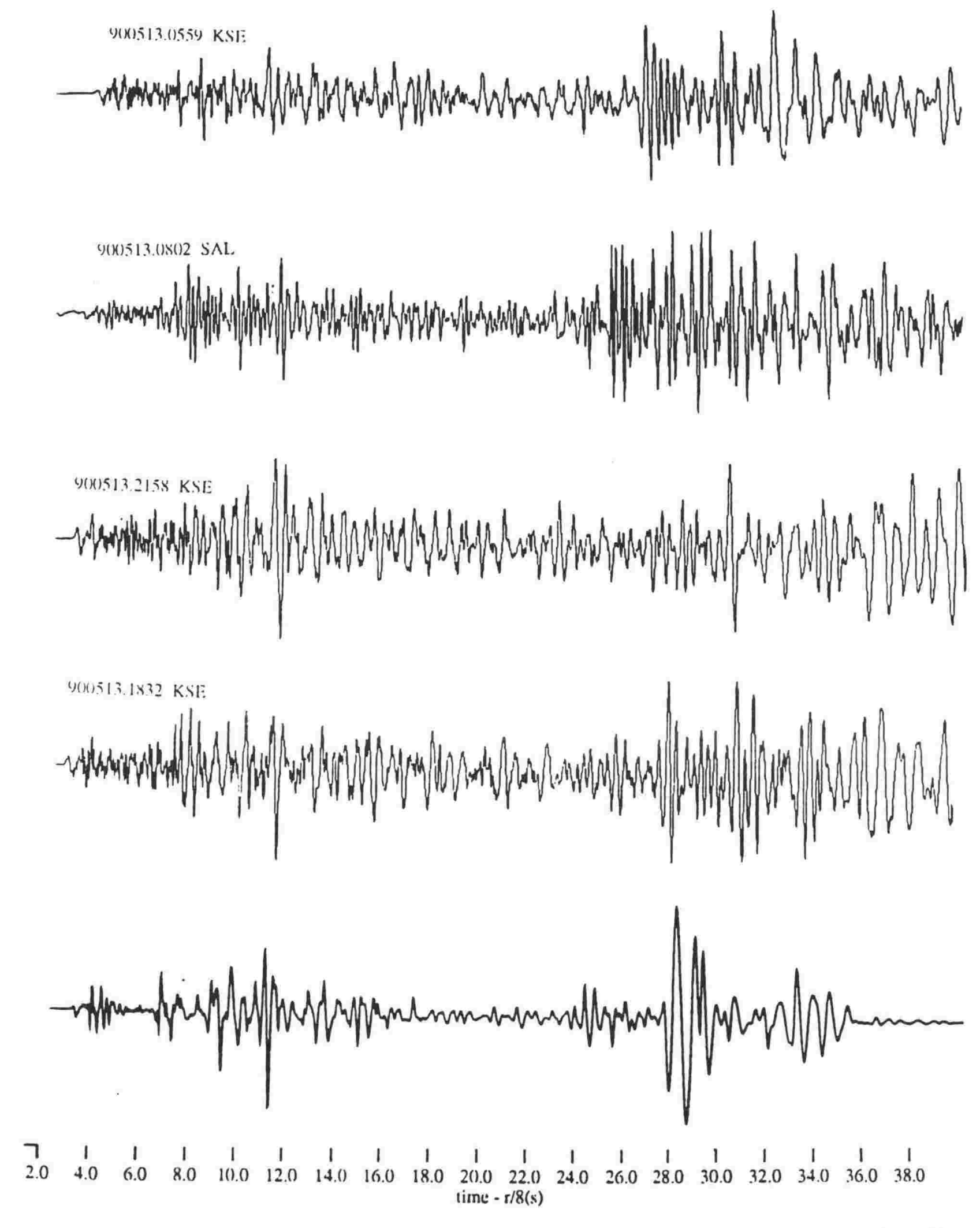

Fig. 5.8 Comparison of vertical component synthetic seismogram (bottom trace) with some of the data. 

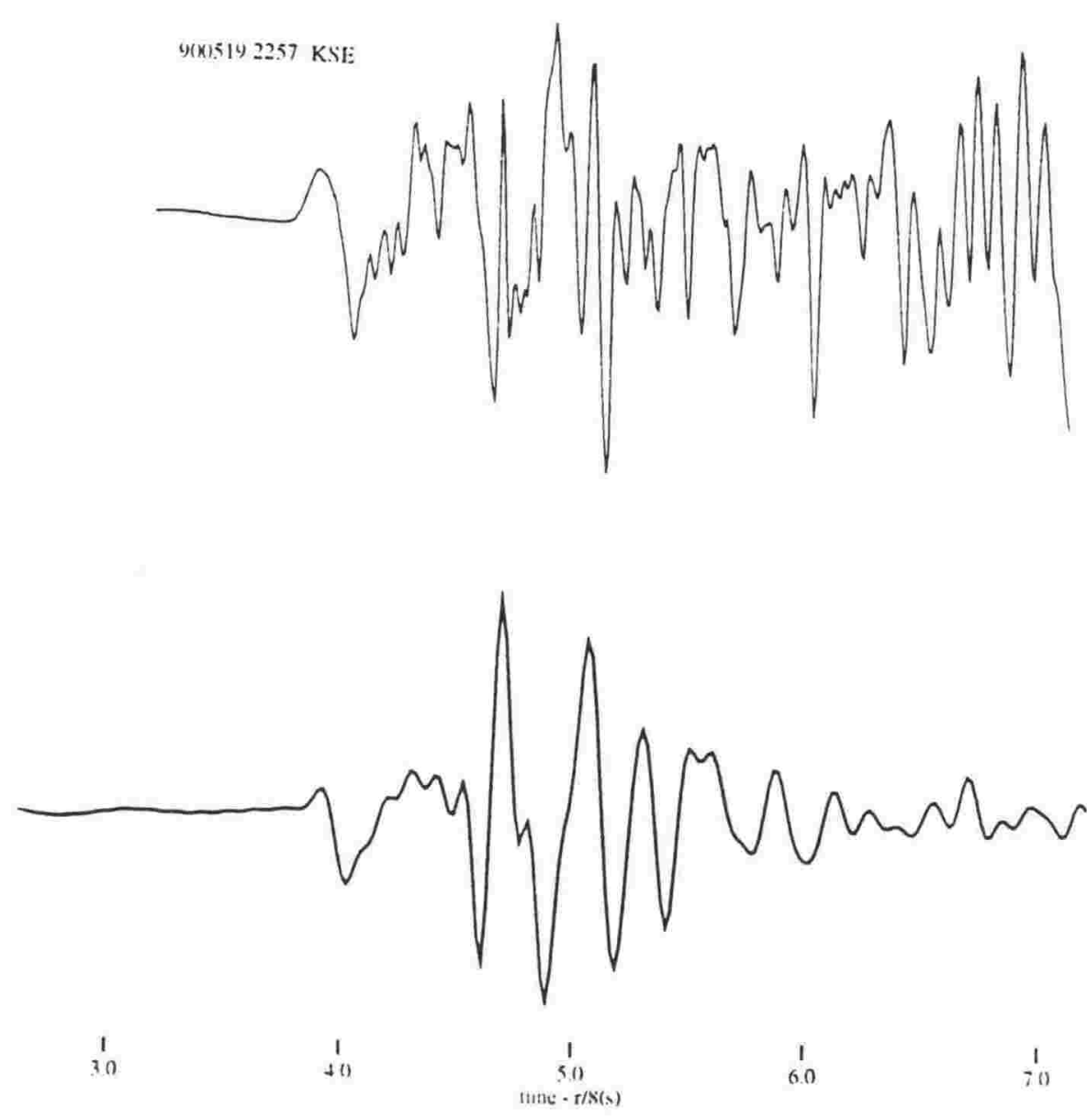

Fig. 5.8b Comparison of first few seconds of synthetic seismogram with real data.

approximation" technique on which Kennett's reflectivity method is based. This procedure breaks down the full seismogram as a sum of more simplified responses. The latter approach is far preferable as it retains information about amplitude, mode conversions, and so on. However, it is still worthwhile to use ray-tracing as a first step of the phase identification.

\subsubsection{Ray-tracing}

Figure 5.9 is an output of the 2-dimensional ray-tracing programme ray84, in which the input velocity model is the one shown in Figure 5.5, and where the synthetic seismogram generated by this model is plotted on the right of the travel-time curve. Head waves along the model boundaries are here approximated by refracted waves, by applying slight velocity gradients in the layers. For simplicity, the whole ray-set is split into two sets. The first (Figure 5.9a) includes rays which propagate in the lower crust and upper mantle of the "subducted slab". It is seen that the major arrivals in the first $4 \mathrm{~s}$ of the seismogram can be correlated with the headwave in layer 7 (first arrival), the headwave in layer 6 , the reflection off layer 7 , the headwave in layer 5 

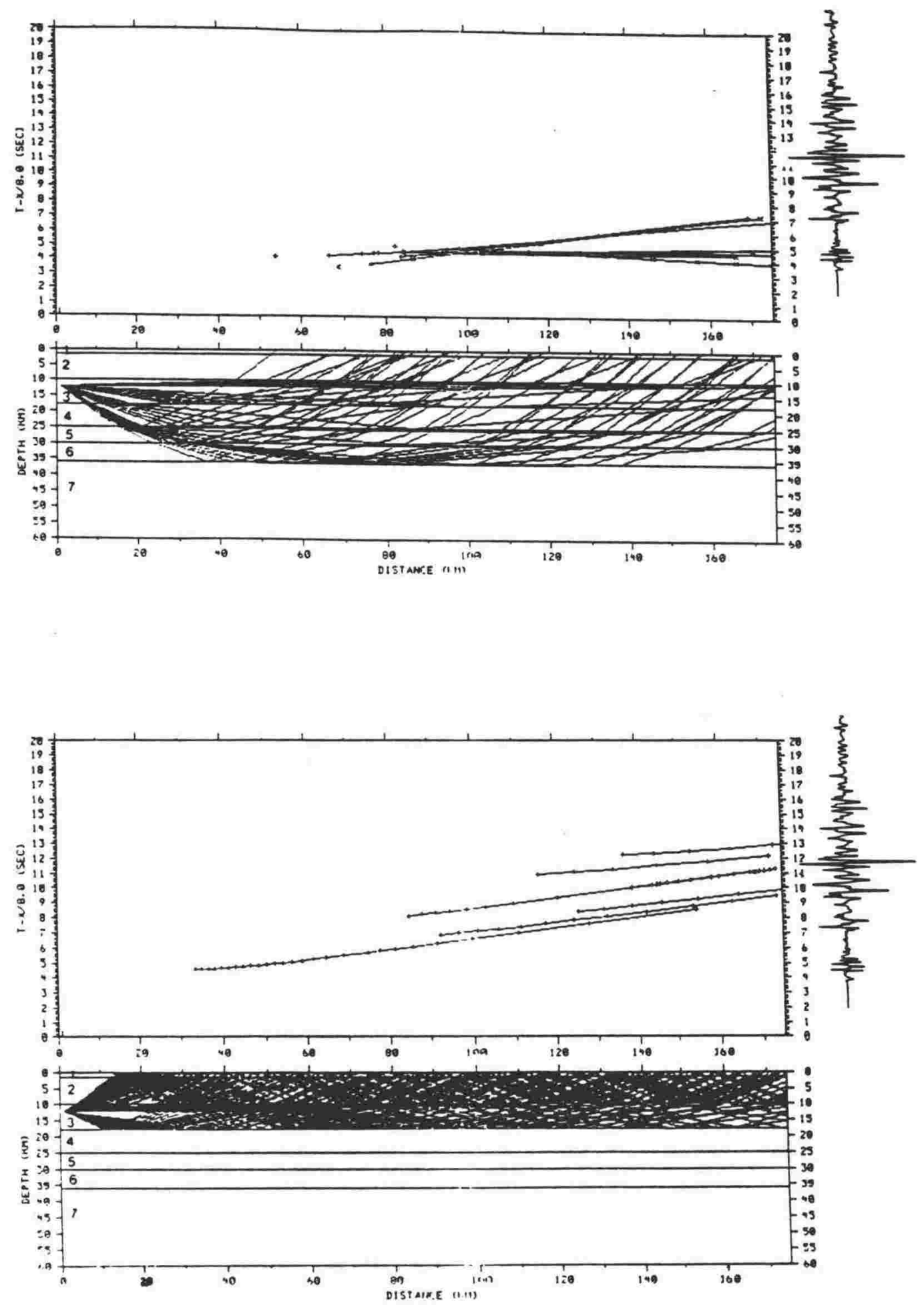

Fig. 5.9 2-dimensional ray-tracing through model MODOI. The synthetic seismogram from the same model is plotted on the side, with the same time scale as the vertical time axis. $x$ are refractions, + are reflections. a) Ray sets through subducted lithosphere, and direct rays; b) Reverberatory phases between the free surface and the subduction interface. 
and the reflection off layer 6 , where the layer numbers are defined in the diagram.

A second set of arrivals is then composed mainly of the reflection in layer 4 , the direct wave and the reflection off layer 5 .

The next arriving wavetrain in the synthetic seismogram is quite well modelled in terms of travel times by a set of multiple crustal phases reverberating between the free surface and the intermediate layers in the vicinity of the subduction interface. This set of rays is illustrated in Figure 5.9b. Note that whatever the actual raypath, all of these crustal phases travel as a group with an apparent velocity of around $6.0 \mathrm{~km} / \mathrm{s}$, at a distance greater than about $100 \mathrm{~km}$. The situation depicted in Figure $5.9 \mathrm{~b}$ is incomplete in that it consists only of $\mathrm{P}$-wave legs, i.e. does not include P/S conversions, and also does not predict which phases would make the largest energy contribution.

\subsubsection{Wavefield approximations}

A far more insightful approach to interpreting the synthetic seismogram is by breaking down the total wavefield into a number of simpler wave types, or groups. This technique is illustrated in Figure 5.10, in which all the seismograms are plotted with the same vertical scale factor.

Figure 5.10a is the full response, as in Figure 5.6. Figure 5.10b is the same seismogram, but with no free surface reflections allowed to contribute to the response. It is clear that while the first section of the seismogram, up to about $5 \mathrm{~s}$, is unaffected, the next part of the wavetrain virtually disappears. This reinforces the hypothesis that the $\bar{P}$ wavegroup contains a major contribution from reverberations at the free surface.

We can analyse this phenomenon further by now allowing free surface reflections but suppressing the return of this surface reflected energy from the "deep" zone, i.e. the structure below the separation interface (Figure 5.10c). It is seen that although the major phases within the $\bar{P}$ group persist, the intensity of this group is slightly decreased, indicating that the lower "boundary" of the crustal waveguide probably includes some layers within the subducted plate, most likely the subducted upper crust, since the model contains a sharp velocity contrast between layer 4 and layer 5 .

Figures 5.10a - c contain the full $\mathrm{P}$ and $\mathrm{S}$ response i.e. allowing $\mathrm{P}-\mathrm{S}$ and $\mathrm{S}-\mathrm{P}$ conversions at each interface encountered. Using the method of wavefield approximations, it is not difficult to obtain a better idea of the relative amounts of $\mathrm{P}$ and $\mathrm{S}$ type motion making up the various parts of the response. For example, Figure 5.10d represents the response when both the source radiation and the surface displacement is made up of only the P-wave. Again, the first 5 seconds are unaffected, however the later part of the $\bar{P}$ group decreases appreciably in amplitude, indicating that it contains a considerable amount of S-wave motion.

When the source radiation consists only of S-wave, and the surface displacement consists only of P-wave (Figure 5.10e), most of the $\bar{P}$ wave group is recovered, but this again disappears 

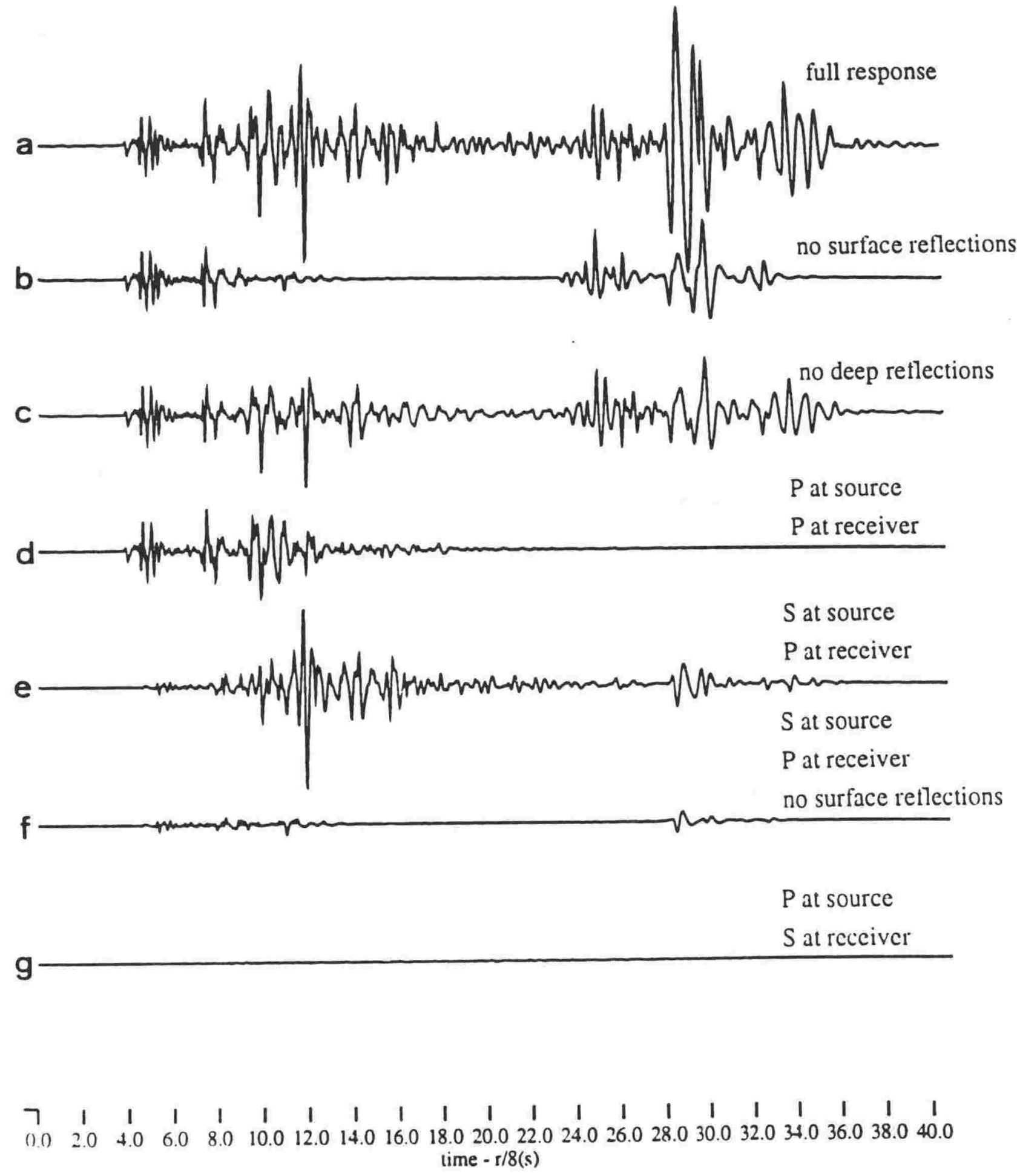

Fig. 5.10 Wavefield approximations for vertical component synthetic seismogram from MODO1. The partial waveforms $a-g$ are explained at the side of each trace. 
once the free surface reflections are eliminated from this response (Figure 5.10f). These two latter wavefield approximations indicate that a large fraction of the $\bar{P}$ energy is contributed by $\mathrm{S}$ to $\mathrm{P}$ conversion at the free surface. $\mathrm{P}$ to $\mathrm{S}$ conversion does not appear to be at all efficient, as evidenced by Figure $5.10 \mathrm{~g}$, which represents the S-wave displacement at the surface from a pure P-wave source.

From this breakdown of the waveform then, it is quite clear what both the origin and the nature of the various parts of the synthetic seismogram are. To summarise, the $P_{h f}$ group is composed of pure P-waves propagating in the lower crust and upper mantle of the subducted lithosphere. An "intermediate" group of arrivals (at reduced time 7 to 9 s) is almost pure P-wave, with some SV-component, and is made up of direct crustal arrivals, as well as some reflections and refractions from the intermediate layers near the subduction interface. The dominant $(\bar{P})$ arrival (reduced time approximately 9 to 17s) is largely made up of reverberations in a crustal waveguide between the free surface and the layers near the subduction interface, and has a high content of P-SV mode conversions, occurring mainly at the free surface. This interpretation agrees well with previous interpretations of $\bar{P}\left(P_{g}\right)$ phases observed in continental areas, and has been made unambiguously clear by the use of wavefield approximations. The conversion of SV to $\mathrm{P}$ does not appear to be at all efficient, and does not contribute significantly to the whole response. The difference in frequency content between $\mathrm{P}_{\mathrm{hf}}$ and $\bar{P}$ observed in the real data can be clearly attributed to the fact that propagation of the two phases takes place through different regions of the lithosphere.

The layer of low velocity at the surface also appears to contribute to some extent to the efficiency of the crustal waveguide. Figure 5.11 compares the responses from a model with no surface low-velocity layer, and 3 models with increasing thicknesses of such a layer. The duration of the $\bar{P}$ phase does appear to increase with increasing thickness of the surface sediments (note the wraparound effect for examples (c) and (d)). This effect has been previously investigated by other workers, e. $g$. Banda $e t a l, 1982$; Olsen $e t a l, 1983$. It is not intended here to make a study of the surface layer, and the values of MOD01 shall be considered satisfactory for the purpose of modelling the lower structure.

\subsection{Modelling velocity features in the subducted upper mantle}

The general waveform features of the synthetic seismogram generated by the basic model MOD01 have been shown to be similar to those of the observed data. Moreover, interpretation of the synthetic seismogram has revealed that identifiable sections of the seismogram can be directly associated with propagation through particular depth regions within the lithosphere, as well as with particular modes of propagation. This is convenient, for it means that the elastic parameters 


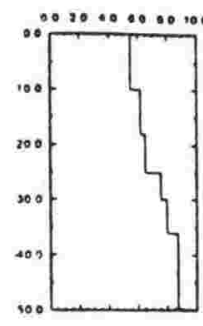

a

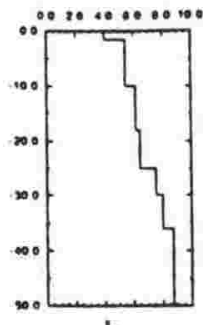

b

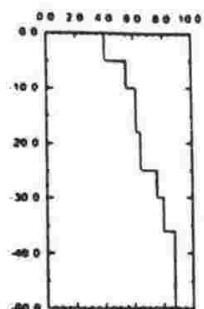

c

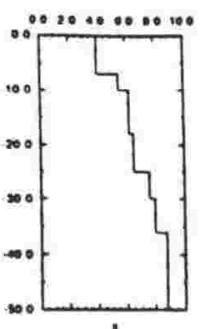

d
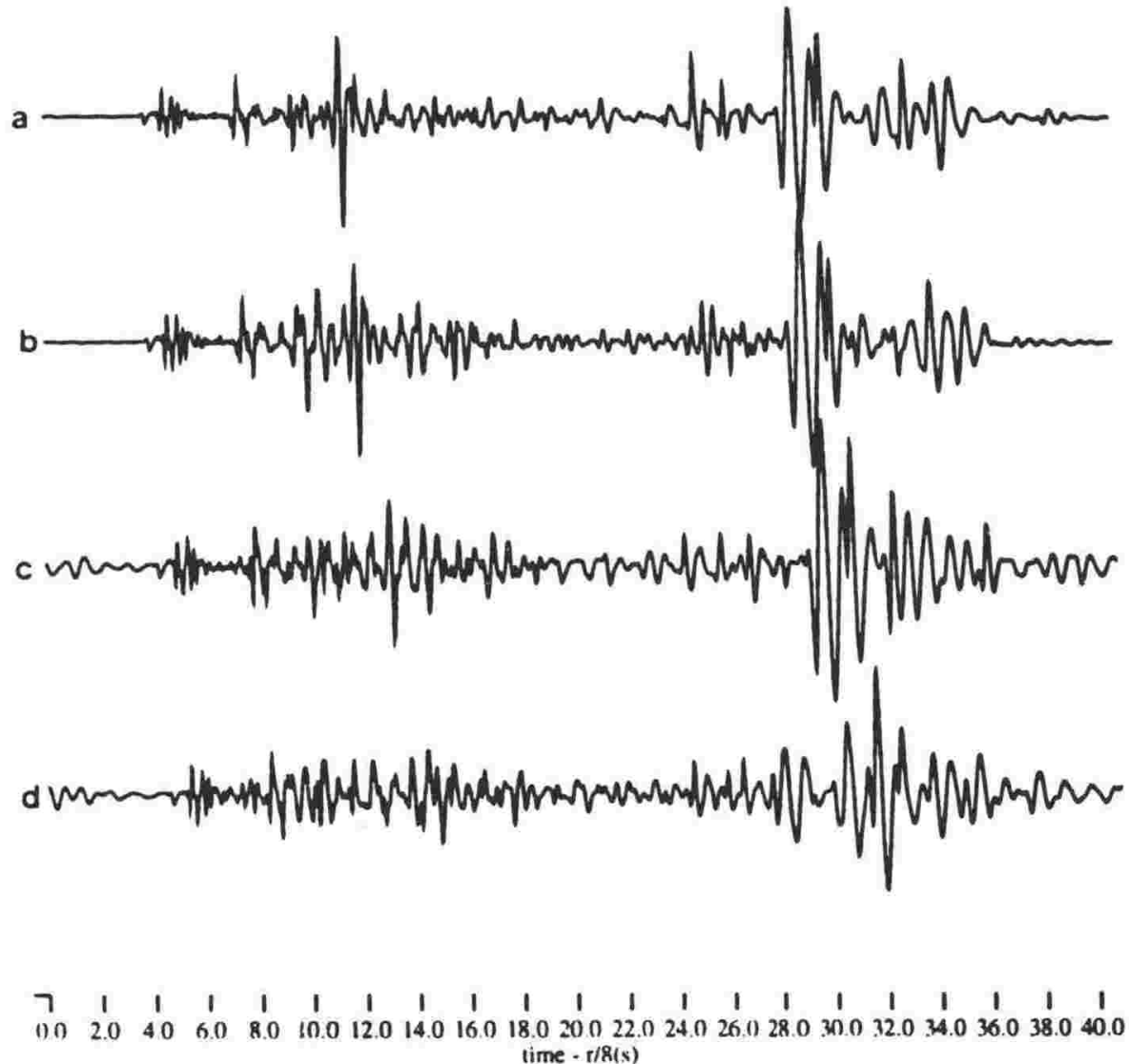

Fig. 5.11 Effect on synthetic seismogram of surface low velocity layer of increasing thickness. Model (a) has no surface layer. Model (b) is identical to MODO1.

in those particular regions of the lithosphere may now be varied, so that their effect on the corresponding part of the synthetic seismogram can be assessed. This will enable us to judge the extent to which particular features of the model can be constrained.

\subsubsection{The presence of "normal" ( $8.0-8.4 \mathrm{~km} / \mathrm{s})$ upper mantle material.}

In Robinson's (1994) joint velocity-hypocentre inversion of the Weber data sets the author 
arrives at a velocity model which has a $7.6 \mathrm{~km} / \mathrm{s}$ layer directly above an $8.77 \mathrm{~km} / \mathrm{s}$ halfspace at a depth of $35 \mathrm{~km}$. Admittedly, not enough deep events were available to constrain the structure at this depth.

From the interpretation of the synthetic seismograms, it is clear that any change in velocity structure at these depths would affect only the first few seconds of the seismogram. This is verified by looking at Figure 5.12, in which several possibilities of velocity structure are explored. Model (a) in Figure 5.12 approximately represents the structure arrived at by Robinson, i.e. an $8.75 \mathrm{~km} / \mathrm{s}$ half-space directly underlying the $7.6 \mathrm{~km} / \mathrm{s}$ layer at depth $35 \mathrm{~km}$, while Model (b) is similar to Model (a) but with the high velocity medium pushed down to $36 \mathrm{~km}$ depth. Models (c) - (e) have layers of $8.0,8.2$ and $8.4 \mathrm{~km} / \mathrm{s}$ respectively introduced between the 7.6 and $8.75 \mathrm{~km} / \mathrm{s}$ media, while Model (f) has an $8.2 \mathrm{~km} / \mathrm{s}$ over an $8.4 \mathrm{~km} / \mathrm{s}$ layer. The P-velocity profiles of the corresponding models are displayed at the top of the figure. Time is reduced at $8.0 \mathrm{~km} / \mathrm{s}$.

In this, and the following examples of modelling some aspects of the upper mantle structure, it is meaningless to attempt a peak-for-peak correlation between real and synthetic data, owing to the diversity of detail in the observed waveforms. Nonetheless, visual examination of Figure 5.12 allows some tentative inferences to be made. The observed complexity in the first few seconds appears to be better modelled by a more layered structure (Figures 5.12(c) - (f)) and the presence of an $8.0-8.4 \mathrm{~km} / \mathrm{s}$ layer contributes in the required manner. An $8.75 \mathrm{~km} / \mathrm{s}$ layer directly underlying the $7.6 \mathrm{~km} / \mathrm{s}$ layer yields a $P_{n}$ pulse that separates too far ahead. It also appears that $8.4 \mathrm{~km} / \mathrm{s}$ for the "normal" upper mantle layer places the $P_{h f}$ phase too early relative to $\mathrm{P}_{\mathrm{n}}$. Therefore, although the waveform modelling and simple visual comparison is not enough to constrain the layer velocities, it appears justified to accept that an $8.0-8.2 \mathrm{~km} / \mathrm{s}$ medium separates the $7.6 \mathrm{~km} / \mathrm{s}$ lower oceanic crust from the high-velocity medium. The comparison of this part of the signal made in Figure $5.8 \mathrm{~b}$, in which the model contains an $8.0 \mathrm{~km} / \mathrm{s}$ layer, also supports this. The structure of MOD01 is therefore an adequate, if not detailed, representation of the velocity profile for the purpose of the modelling.

\subsubsection{Depth of high velocity medium}

Once an upper mantle velocity of $8.0 \mathrm{~km} / \mathrm{s}$ is assumed, the depth at which the $8.75 \mathrm{~km} / \mathrm{s}$ medium begins can be constrained to a somewhat better extent. The effect is demonstrated in Figure 5.13, in which the depth to the top of the high-velocity medium is varied from $32 \mathrm{~km}$ to 48 $\mathrm{km}$. At a depth of $32 \mathrm{~km}$, the $P_{n}$ pulse separates too far ahead of the rest of the signal, while if the high-velocity medium is deeper than about $40 \mathrm{~km}$, then, at the distance of $175 \mathrm{~km}$, the 8.0 $\mathrm{km} / \mathrm{s}$ head wave arrives earlier.

Admittedly, as regards the time separation between the $\mathrm{P}_{\mathrm{n}}$ and $\mathrm{P}_{\mathrm{hf}}$ signals, there is a trade-off between the depth of the high-velocity medium and the velocity of the medium directly overlying it. An increase in the P-velocity in the overlying medium, making the $\mathrm{P}_{\mathrm{hf}}$ phase arrive early, could be offset by a shallowing of the high velocity medium. However, when looking at 
waveforms, it is not enough to compare only travel times. The thickness of the layers in this particular region also affects the pulse shapes and amplitudes (Figure 5.13) presumably due to the varying incident angles, so that it is better to be guided by the best-fitting waveform.

While emphasising once more that a detailed analysis cannot be strictly applied to a comparison with the real data from a 3-dimensional structure, it is probably justified to claim that any high velocity material must begin at a depth of about $34-40 \mathrm{~km}$ in the flat-layered model, which is consistent with the average depth in the 3-dimensional model used in the Complete Ray Tracing (Chapter 3). Modelling of a closely-spaced refraction profile, such as the Hikurangi Margin experiment, should give much better constraint over such details.

\subsubsection{Different velocity profiles for a layer of high velocity}

So far, the high velocity medium has been represented in all models as a half-space for simplicity. In the real earth, however, it is unlikely that such high velocities, normally found closer to $400 \mathrm{~km}$ depth, would persist from a shallow depth to beyond a layer of finite thickness. In the following, the effect of a layer of P-velocity $8.75 \mathrm{~km} / \mathrm{s}$ of various thicknesses is studied. The effect of the shape of the velocity profile above and below the layer, with the introduction of velocity gradients, is also investigated.

In Figure 5.14, which illustrates the effect of thickness, it can be seen that a significant head wave of the shape and amplitude of that observed in the data does not appear to develop until the layer is at least $4 \mathrm{~km}$ thick.

With the $8.75 \mathrm{~km} / \mathrm{s}$ layer having sharp velocity discontinuities at its upper and lower boundaries, it is observed that the head wave pulse shape is modified by a sharp positive peak immediately following the downward swing of the pulse. This is interpreted as being a reflection from the underside of the layer. This interpretation is justified by noting that when the sharp velocity contrast at the lower boundary is replaced by a constant, negative velocity gradient, the reflection is suppressed and the $P_{n}$ pulse closely resembles that from an $8.75 \mathrm{~km} / \mathrm{s}$ half-space, and is also more similar to that seen in the data (see Figure 5.3).

Figure 5.15 investigates the effect of replacing the first-order velocity discontinuities bounding the high-velocity layer by velocity gradients above and below the layer. A sharp gradient on the underside, as in Figure 5.15(e) has the same effect as a first-order discontinuity, namely the contamination of the $P_{n}$ pulse with the "reflection" from the underside. The gradient above the layer is more difficult to ascertain because of its apparently smaller influence (compare Figures 15(b) and (d)). The conclusion that can be drawn here is that a velocity profile of the type shown in Figure 5.15(d) is not inconsistent with the observations.

\subsubsection{Conclusions}

In conclusion, an upper mantle P-velocity model that generates a satisfactory response compared with the real data would have a high-velocity $(8.75 \mathrm{~km} / \mathrm{s})$ layer, of thickness greater 

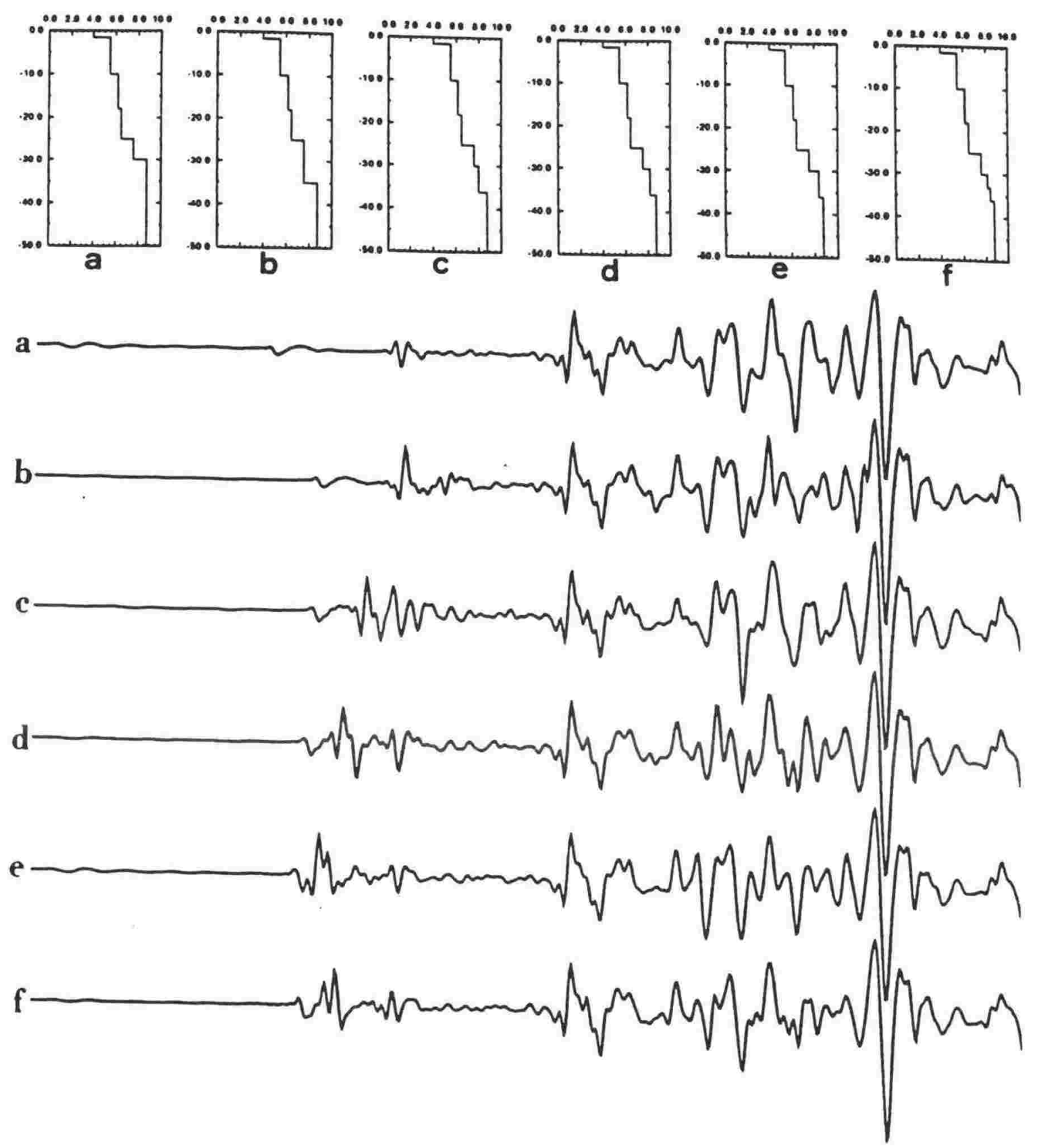

$\begin{array}{ccccccccccccc}7 & 1 & 1 & 1 & 1 & 1 & 1 & 1 & 1 & 1 & 1 & 1 & 1 \\ 0.0 & 1.0 & 2.0 & 3.0 & 4.0 & 5.0 & 6.0 & 7.0 & 8.0 & 9.0 & 10.0 & 11.0 & 12.0 \\ & & & & & & \text { lime } \cdot r / 8(\mathrm{~s}) & & & & & & \end{array}$

Fig. 5.12 Effect of different velocity profiles above the high velocity medium. 

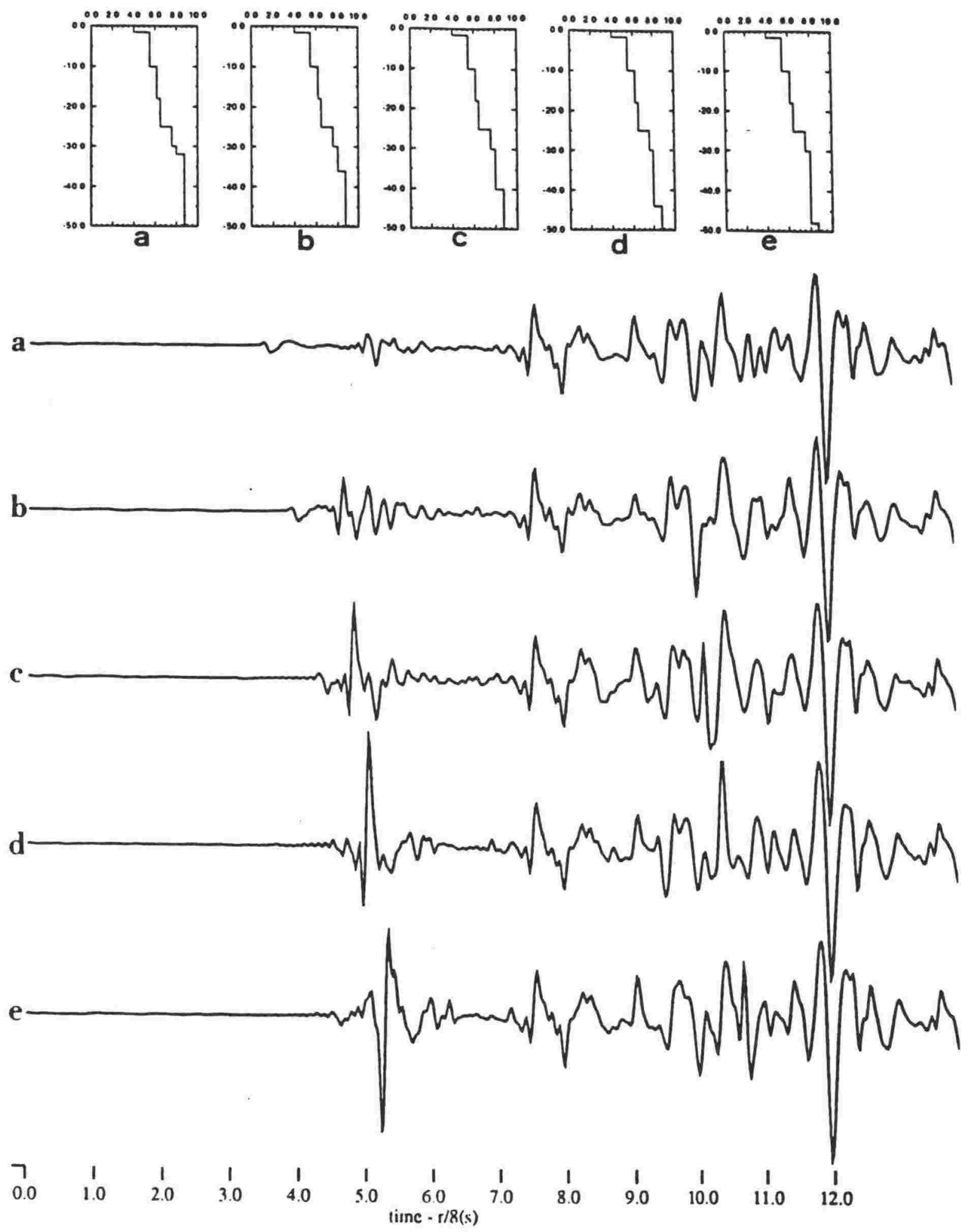

Fig. 5.13 Synthetic seismograms from models identical to MODOI but in which the high-velocity medium begins at varying depth a) $32 \mathrm{~km} \mathrm{b)} 36 \mathrm{~km} \mathrm{c)40} \mathrm{km} \mathrm{d)44} \mathrm{km} \mathrm{e}) 48 \mathrm{~km}$. 


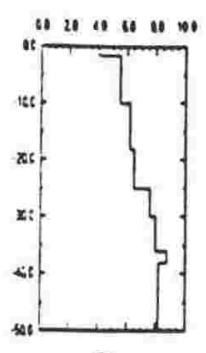

a

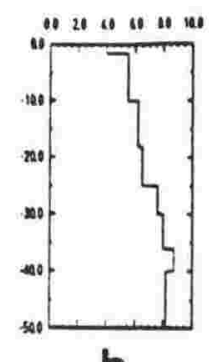

b

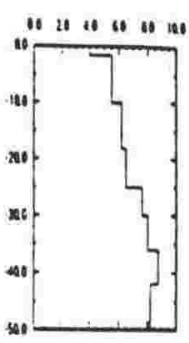

C

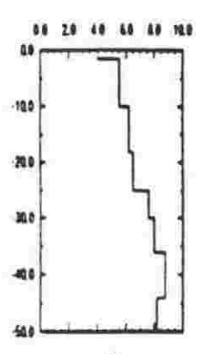

d

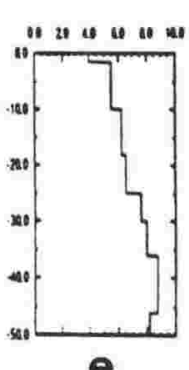

e
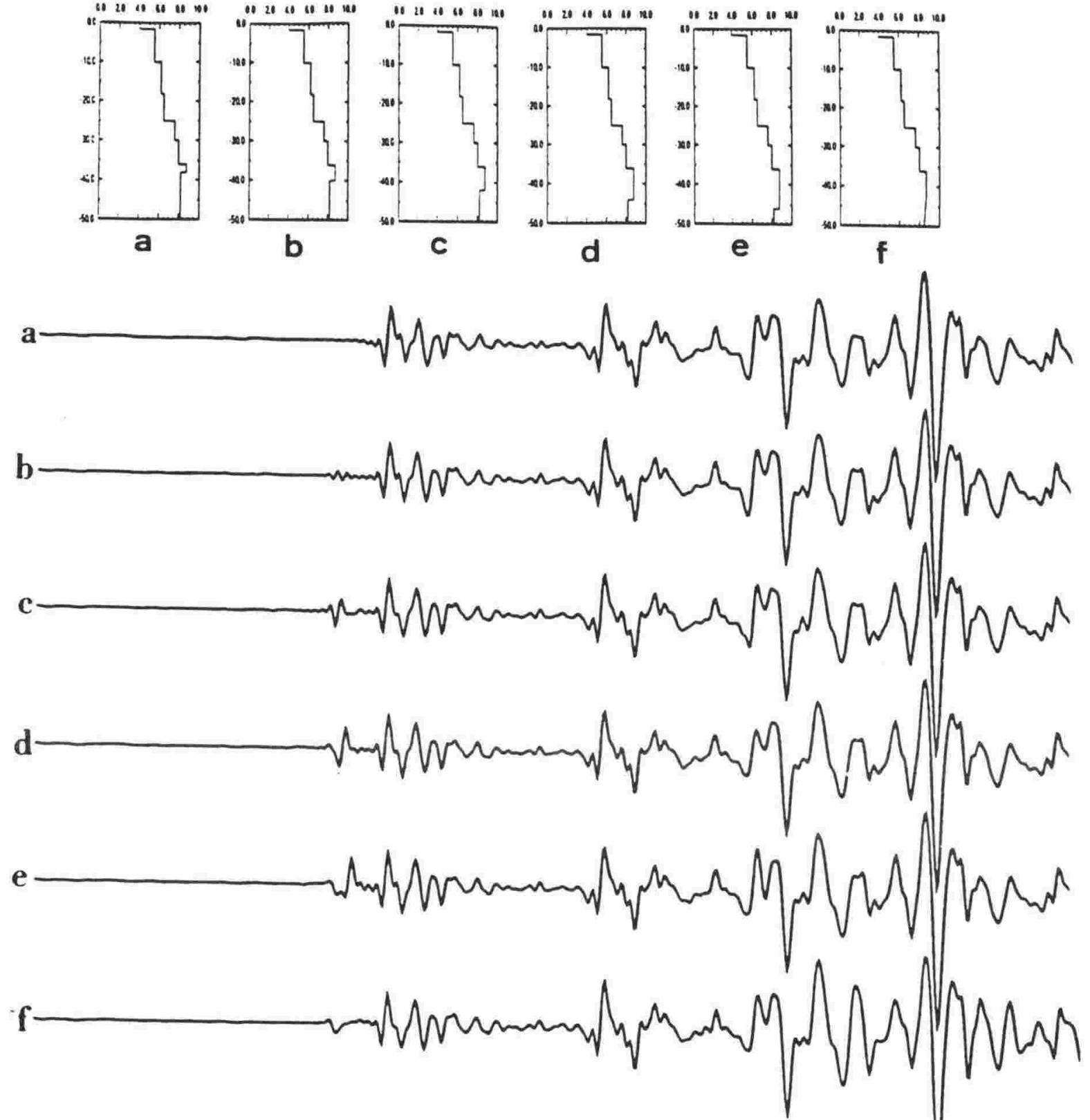

$\begin{array}{ccccccccccccc}7 & 1 & 1 & 1 & 1 & 1 & 1 & 1 & 1 & 1 & 1 & 1 & 1 \\ 0.0 & 1.0 & 2.0 & 3.0 & 4.0 & 5.0 & 6.0 & 7.0 & 8.0 & 9.0 & 10.0 & 11.0 & 12.0\end{array}$

Fig. 5.14 Synthetic seismograms from models identical to MODO1, but in which the high-velocity half-

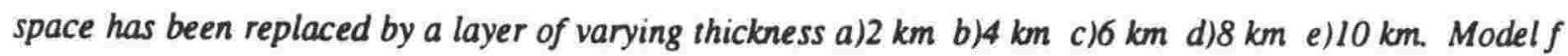
has a negative velocity gradiens bounding the bottom of the high velocity layer. 

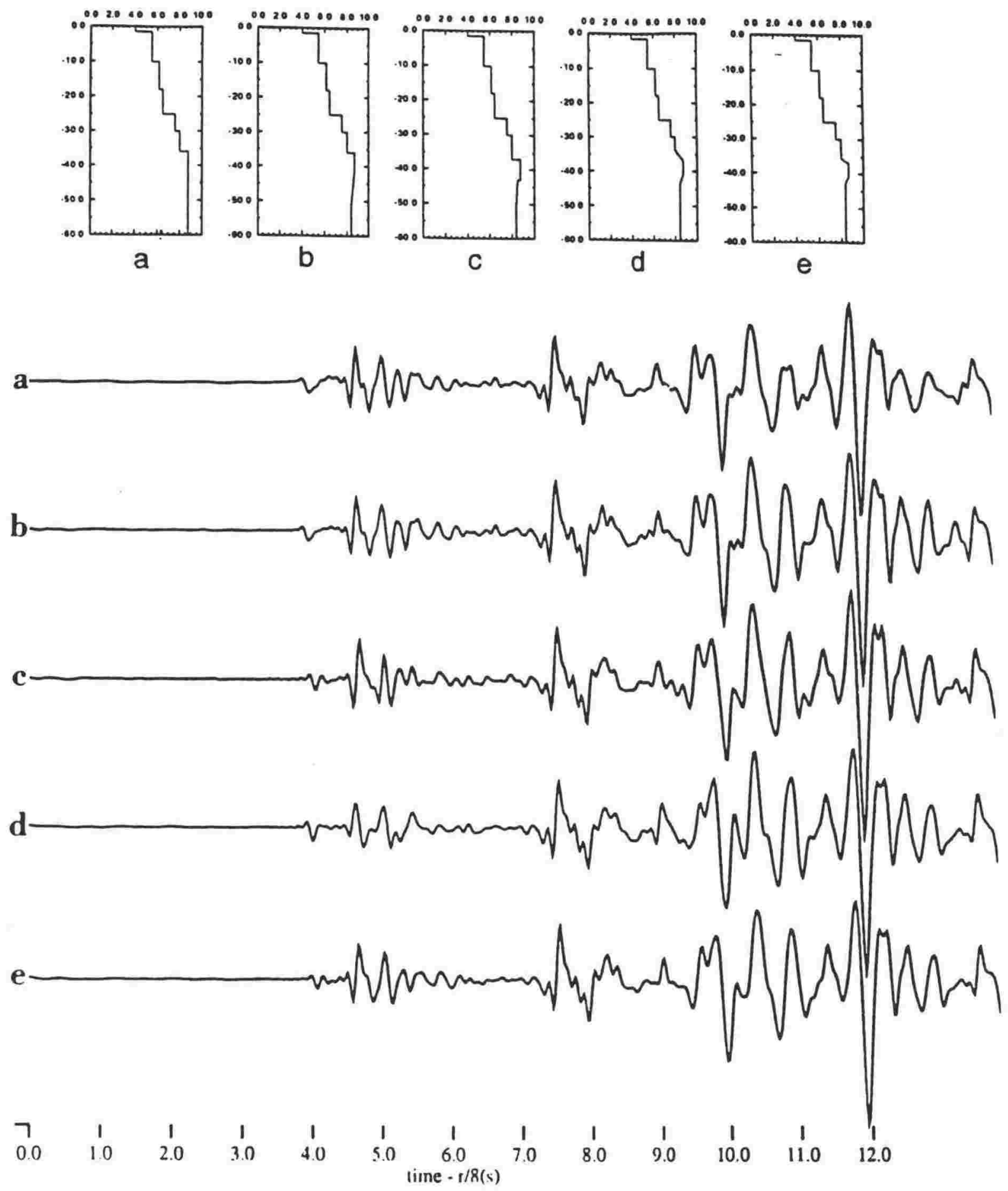

Fig. 5.15 Synthetic seismograms showing the effect of replacing the first order velocity discontinuities bounding the high-velocity layer of Figure 5.14 by positive and negative velocity gradients. The corresponding velocity profiles are shown at the top. 
than $4 \mathrm{~km}$, possibly with a gradual increase and decrease of velocity above and below the layer respectively. This "tooth" would lie at a depth of approximately 18-20 km below the surface of the subducted plate, embedded in "normal" upper mantle material of P-velocity $8.0-8.2 \mathrm{~km} / \mathrm{s}$.

Such high velocity layers have been referred to in several cases in the literature. A layer of P-velocity $8.6 \mathrm{~km} / \mathrm{s}$ within an $8.2-8.4 \mathrm{~km} / \mathrm{s}$ upper mantle has been found in the northwestern Pacific lithosphere off Japan in long-range refraction experiments (Asada et al, 1983; Shimamura and Asada, 1976; Asada and Shimamura, 1976). Hales et al (1970) find clear evidence from refraction profiles of an $8.77 \mathrm{~km} / \mathrm{s}$ layer underlying an $8.0 \mathrm{~km} / \mathrm{s}$ mantle at a depth of about $57 \mathrm{~km}$ in the Gulf of Mexico. Fuchs and Schulz (1976) propose a model for the subcrustal lithosphere containing thin high-velocity layers. Low-frequency "tunnel waves" through such layers are studied both by numerical experiments as well as through observations from explosion data in continental areas. The authors attribute these layers (in the depth range $30-150 \mathrm{~km}$ ) to the presence of strongly anisotropic materials, such as olivine, with a preferred orientation. Sverev and Yaroshevskay (1987) use deep seismic sounding results from the South Atlantic ocean to establish the presence of high velocity layers $(8.4-8.6$ and $8.8 \mathrm{~km} / \mathrm{s})$ in the subcrustal mantle, beginning at depths of around $10 \mathrm{~km}$. The $8.8 \mathrm{~km} / \mathrm{s}$ layer, present under the Angola abyssal plain, occurs at around $25 \mathrm{~km}$ depth, and is also attributed to strongly anisotropic material. Bean and Jacob (1990) derived an anisotropic structure for the lithosphere under the North Irish Sea, containing layers of anisotropic material separated by isotropic ones. The high-velocity layers (which occur when viewed along the fast axis) do not consist of first-order velocity discontinuities, but are bounded by positive and negative velocity gradients. The relationship between these high velocity layers and upper mantle anisotropy is discussed in the final chapter.

\subsection{The effect of a low velocity layer at the top of the subducted slab}

\subsubsection{General effect of an oceanic low-velocity layer}

The presence of a low-velocity layer at the surface of the subducted slab along the East Coast of the North Island has been detected by several authors, and interpreted to be caused by oceanic sediment being subducted with the Pacific plate. Bannister (1988), in his inversion of earthquake travel times in the Hawkes Bay area arrives at a velocity model containing a $3 \mathrm{~km}$ thick layer of P-velocity $4.8 \mathrm{~km} / \mathrm{s}$. Xun (1992) analysed travel times from an aftershock sequence off Cape Palliser, at the southeasternmost tip of the North Island, and concluded that there must be a layer, approximately $1.4 \mathrm{~km}$ thick, and of P-velocity $3.6 \mathrm{~km} / \mathrm{s}$ separating the overlying crust from the subducting Pacific crust. Davey et al (1986) and Lewis and Davey (1987), using mainly results from seismic reflection profiling off the southeast coast of the North Island, detect a 
velocity reversal, interpreted as a $1 \mathrm{~km}$ thickness of consolidated oceanic sediment going under the convergent margin with the underlying oceanic basement. Lewis and Davey (1987) claim that the decollement corresponding to the subduction interface is not at the boundary between the accretionary sediment and the oceanic basement, but within the sedimentary column.

It thus followed that one feature of the velocity profile which could be investigated by waveform modelling was the presence of a layer of reversed velocity at the subduction interface. It was intended to observe the effect of such a layer on the synthetic seismograms, conclude whether such a layer might be present or not, and perhaps make some constraints about its thickness and P-velocity.

Figure 5.16 shows a velocity profile containing a layer of subducted sediment ( $2 \mathrm{~km}$ of 4.8 $\mathrm{km} / \mathrm{s}$ material). We shall refer to this model as MOD02. The figure shows the full response at $175 \mathrm{~km}$, together with the profile and corresponding response for the basic model MOD01, for comparison. Figure 5.17 shows the response from the same velocity model, but broken down into wavefield approximations in an attempt to better interpret the significance of the synthetic seismogram.

The effect of the low-velocity layer (Figure 5.16) is quite drastic, and the resultant waveform fundamentally different to that from the basic model. The dominant phase of the $\mathrm{P}$ wavetrain now shifts to the later part of the P-coda, and has a low frequency reverberatory character. The amplitude of the earlier $\bar{P}$ phase, which was dominant in the seismogram from MOD01, is now suppressed.

The wavefield approximations (Figure 5.17) are of greater signifigance, providing further insight into the meaning of this effect. The elimination of surface reflections (Figure 5.17b) causes the dominant late wavetrain to disappear almost completely, leading one to postulate that this wavetrain is also a reverberatory phase, with the free surface as one boundary of a waveguide. Figure $5.17 \mathrm{c}$ is the response when free surface reflections are included, however the return of this reflected energy from the "deep" zone, i.e. the region below the subduction interface, is eliminated. Again the late part of the dominant wavetrain of Figure 5.15a is highly reduced, suggesting that this wavetrain contains a large proportion of interaction with the layers below the separation level, most likely the sediment layer in particular. Figure 5.17d - e, in which surface reflections are included, demonstrate the importance of phase conversions in the dominant wavetrain. With only $P$ waves at the source and $P$ waves at the receiver, very little energy arrives after the first 4 seconds, indicating that most of the high-amplitude arrivals consist of mode conversions between $P$ and $S$. Figures 5.1e and 5.1f together suggest that $P$ to $S$ conversion is the more efficient and provides the largest contribution to the dominant phase, which is composed mainly of S-wave motion. This is in contrast to the model without the low-velocity layer (MOD01) in which it was inferred (Figure 5.10) that S to $\mathrm{P}$ conversion was the major contributor to the amplitude of the $\bar{P}$ phase.

Figure 5.18 is a long-range profile $(200-300 \mathrm{~km})$ of the same velocity model response, 


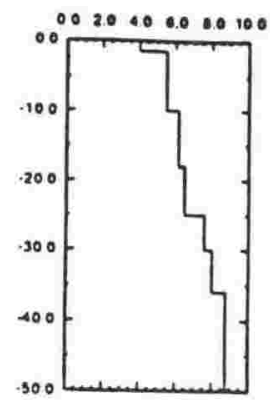

a

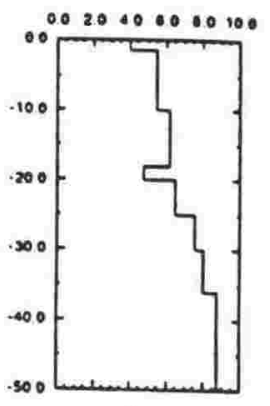

b
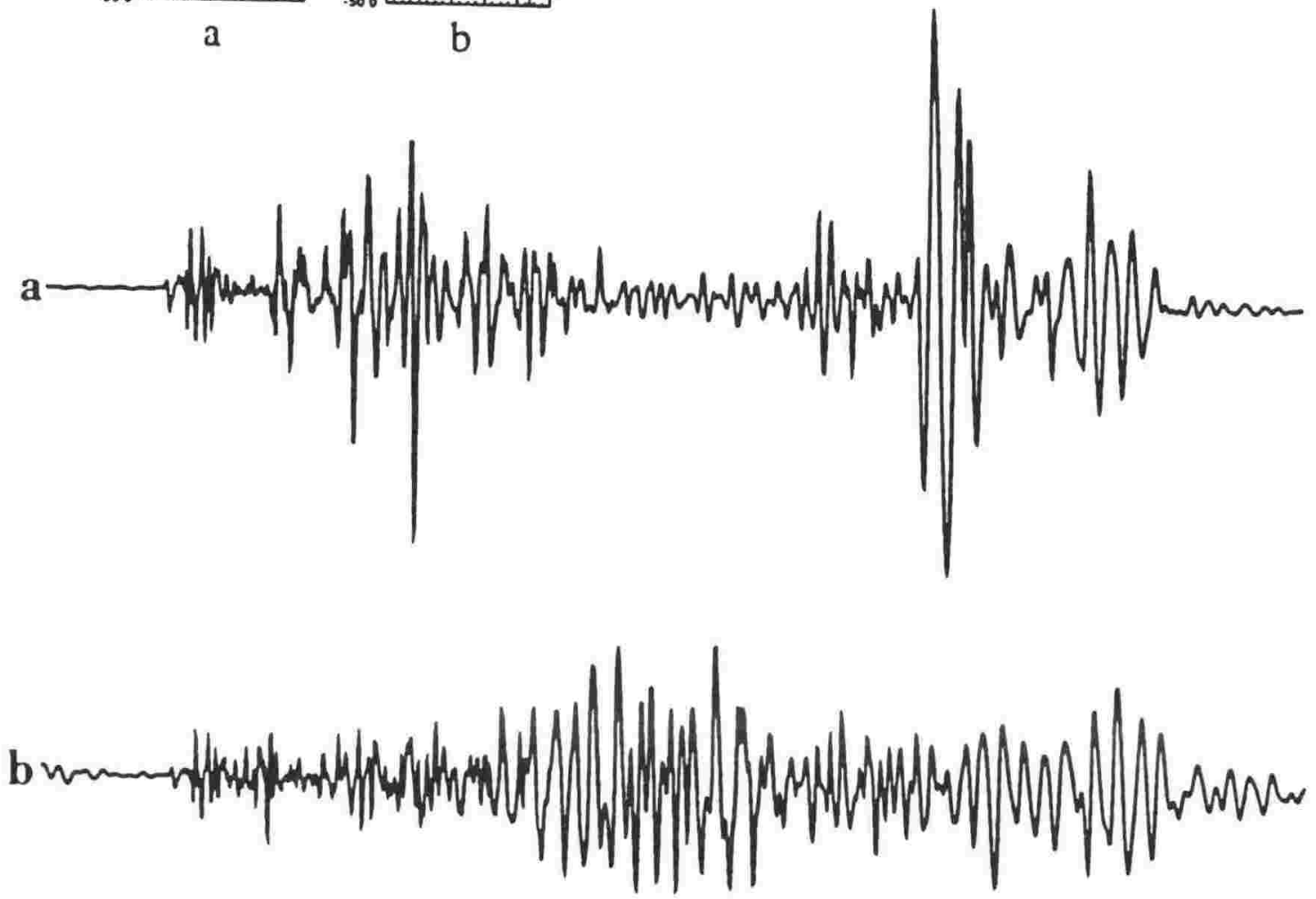

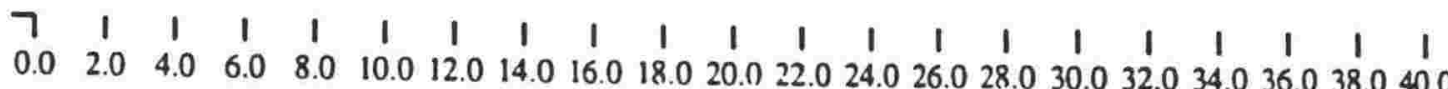
time $-r / 8(s)$

Fig.5.16 Synthetic seismogram from a model containing a $2 \mathrm{~km}$-thick layer of low-velocity $(4.8 \mathrm{~km} / \mathrm{s})$ material at the top of the subducted slab, compared with that from MODO1. The profile for the new model is shown at the top. 


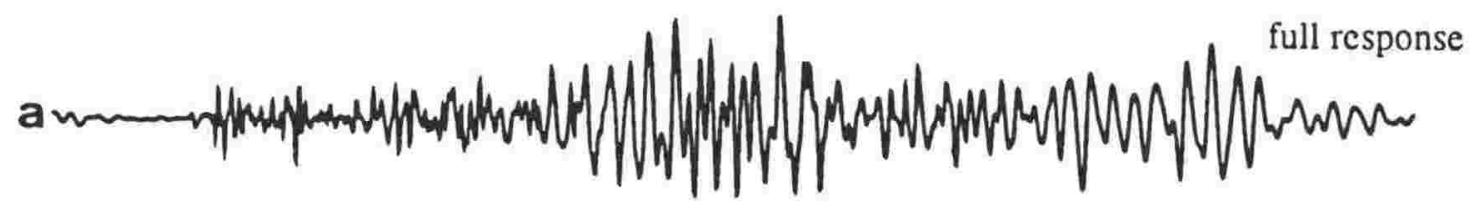

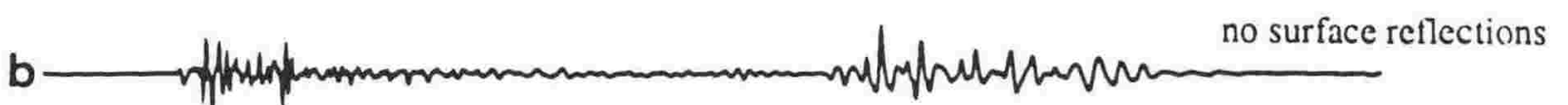
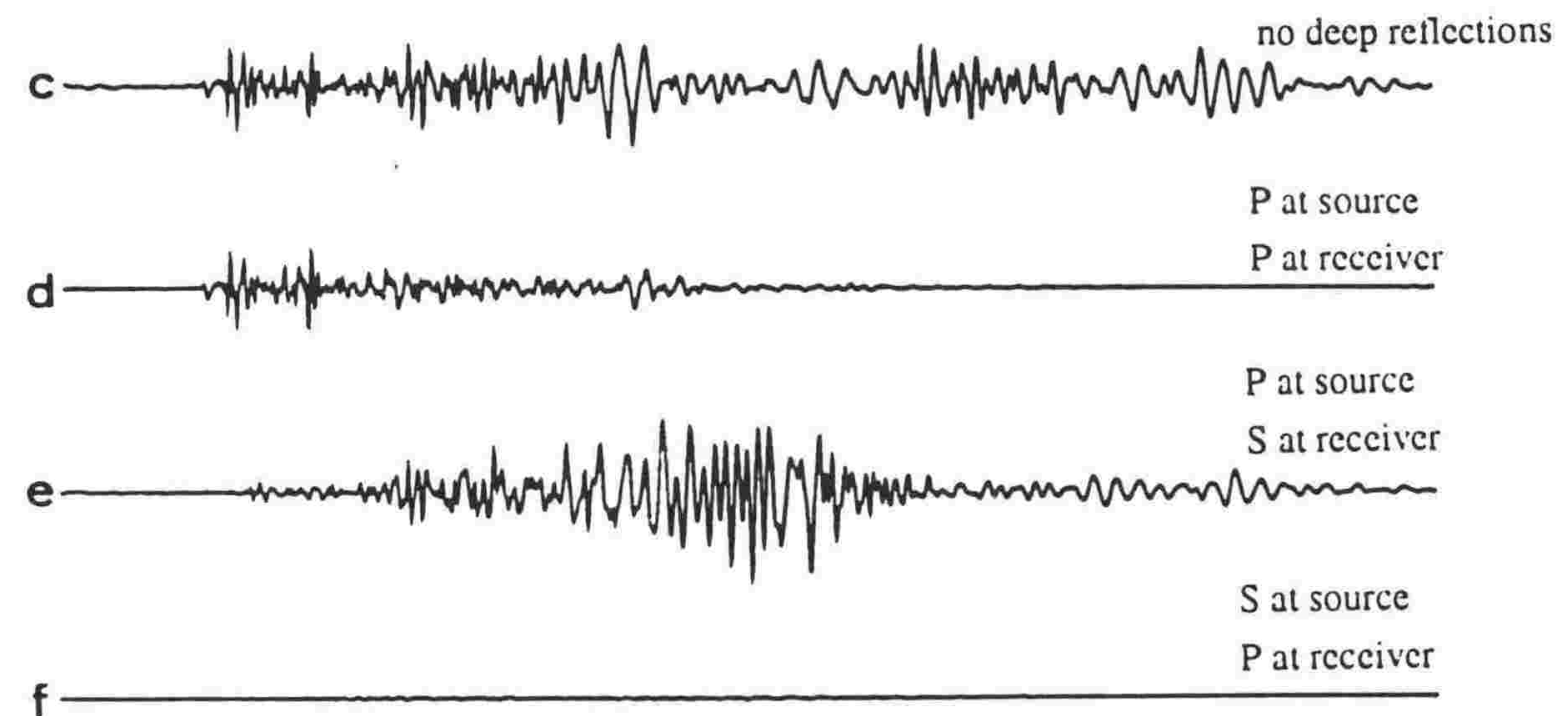

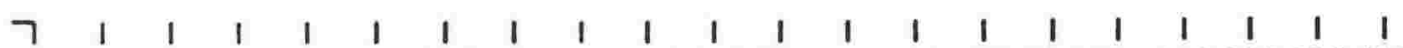

$\begin{array}{llllllll}0.0 & 2.0 & 40 & 6.0 & 8.0 & 10.012 .014 .016 \cap 18.020 .022 .024 .026 .028 .030 .032 .034 .036 .038 .040 .0\end{array}$ time $\cdot r / 8(s)$

Fig. 5.17 Wavefield approximations for the synthetic seismogram of Figure 5.16. 


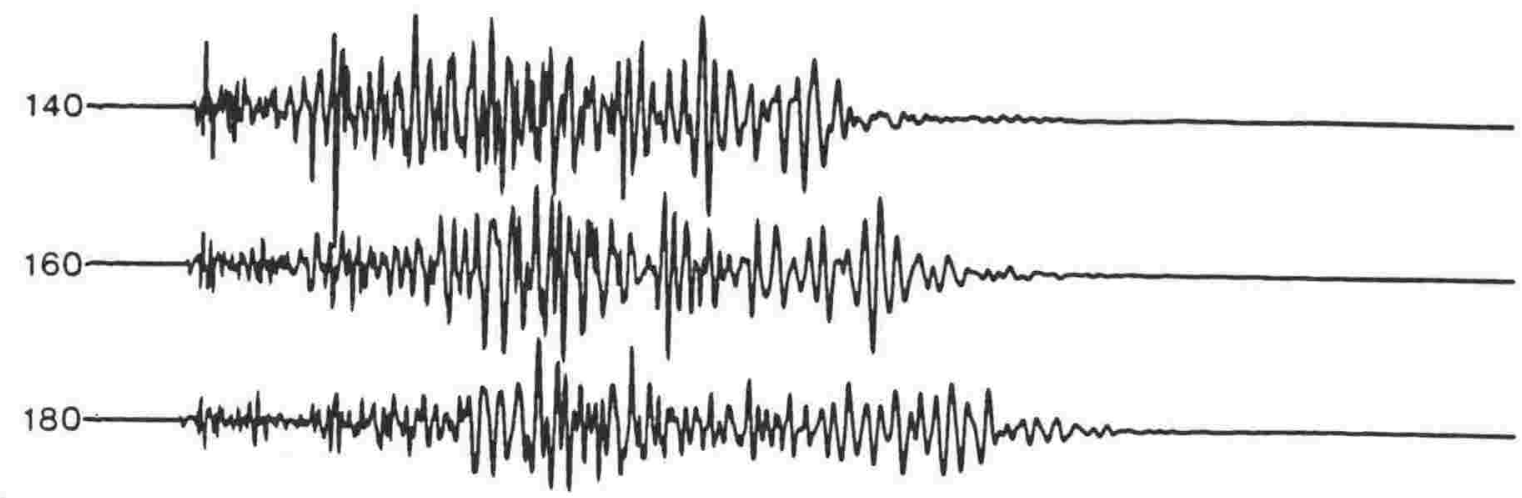

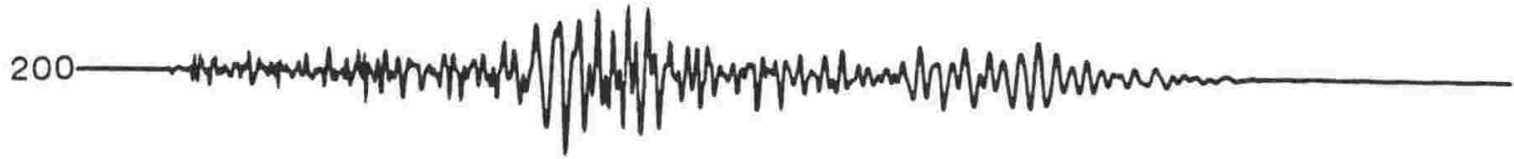

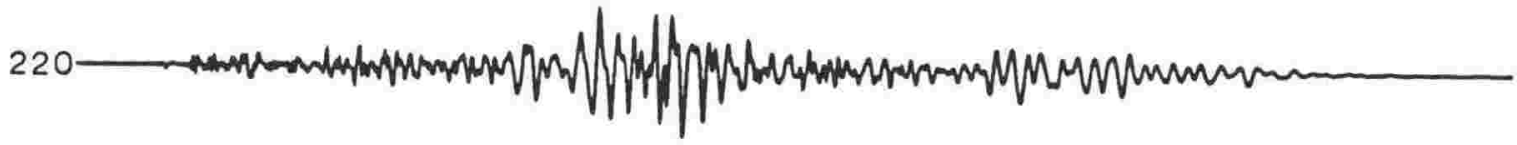

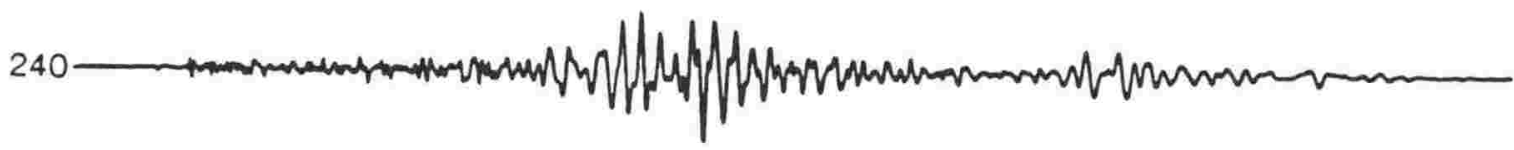

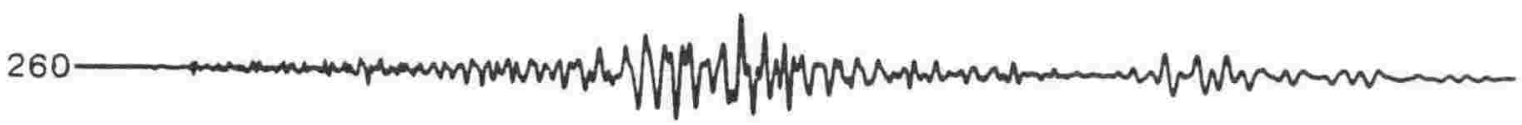
280 300

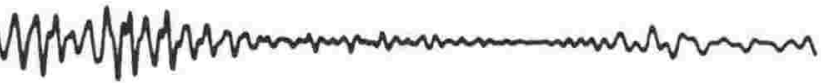

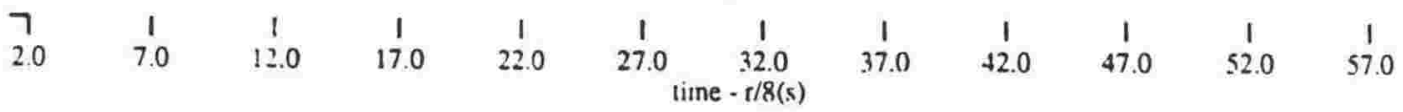

Fig. 5.18 Long-range profile for the synthetic seismogram of Figure 5.16 
demonstrating the long distances over which such wavetrains will propagate. The most likely interpretation is that the low-velocity, subducted sediment layer enhances the efficiency of the crustal waveguide. The late arrival of the wavetrain, as compared with that of the $\bar{P}$ phase of MOD01 at the same distance, is probably due to the fact that most of the energy travels as $S$ waves, since we have seen that $\mathrm{P}$ to $\mathrm{S}$ conversion is the most effective.

Now it is clear that such effects are not observed in the Weber data set recorded at Wellington (see Figure 5.1). However, it is interesting to note that a similar kind of wavetrain is observed for the same Weber aftershocks recorded on National Network stations PUZ and HBZ along the East Coast of the North Island, north of Hawkes Bay. Figure 5.19 shows the location of the stations mentioned and the waveforms at these stations from one of the Weber aftershocks. The waveforms are seen to have the same features as the synthetic ones in the long range profile at the corresponding distances. Finally, Figure 5.19 also shows, for comparison, the basic model synthetic seismogram computed at $300 \mathrm{~km}$ range, showing the difference between the main energy arrival times at these distances.

Figure 5.20 shows more data, this time from an earthquake which occurred in Waipawa (40.0S, 176.8E) on 15 April 1991 (22:25:49 UT) during the Hikurangi Margin refraction experiment. This earthquake triggered most stations along the line. Its depth was recorded by the Seismological Observatory at $26.4 \mathrm{~km}$. The map in Figure 5.20 shows the position of some stations along the refraction line and the seismograms from three of these stations, E06, E05 and E04. It is seen that the seismograms also appear to have the same P-coda characteristics as in the synthetic seismograms from MOD02.

It is natural at this stage to question whether one can detect any significant change in the nature of the synthetic seismogram with a change in the nature of the low velocity layer, in other words, its thickness and the P-velocity within it. Not enough data are available here to enable reliable modelling of such features, so, in the following sections, the effect of varying these two parameters is only briefly investigated. A more detailed analysis of seismograms from East Coast wavepaths showing such behaviour would be a good topic for further research.

\subsubsection{Effect of layer thickness}

Figure 5.21 shows the effect of introducing an increasing thickness of a layer with Pvelocity $4.8 \mathrm{~km} / \mathrm{s}$, starting from a very thin layer of only $10 \mathrm{~m}$, and up to a thickness of $3 \mathrm{~km}$. It appears that the large amplitudes in the late P-coda do not properly develop unless the low velocity layer is about $1 \mathrm{~km}$ thick. Increasing the thickness of the layer does bring about some change in the character of the reverberations, but this change is not pronounced enough to constrain the thickness with any accuracy.

\subsubsection{Effect of layer P-velocity}

Figure 5.22 investigates the effect of varying the P-velocity within the low-velocity layer. In 


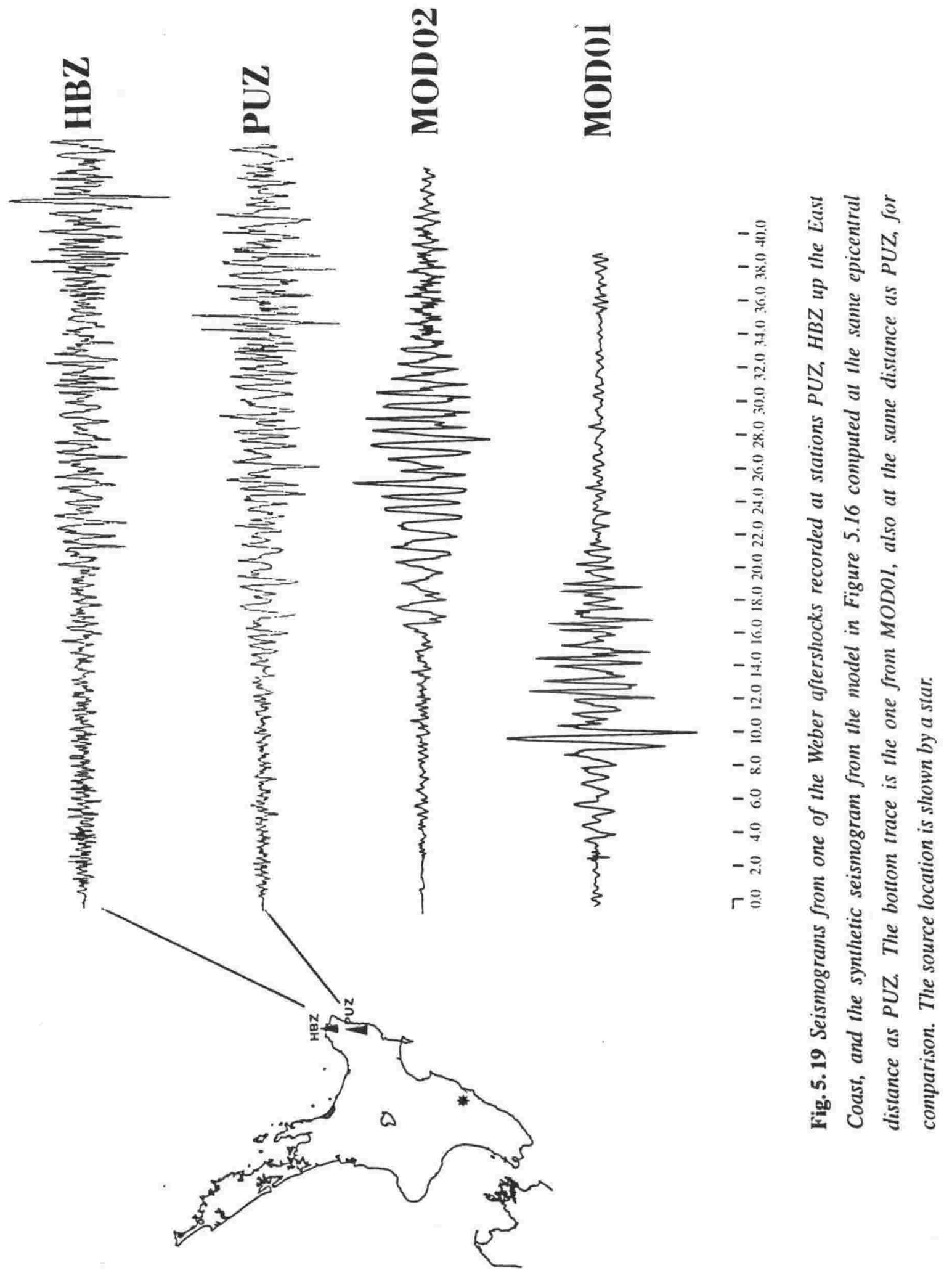




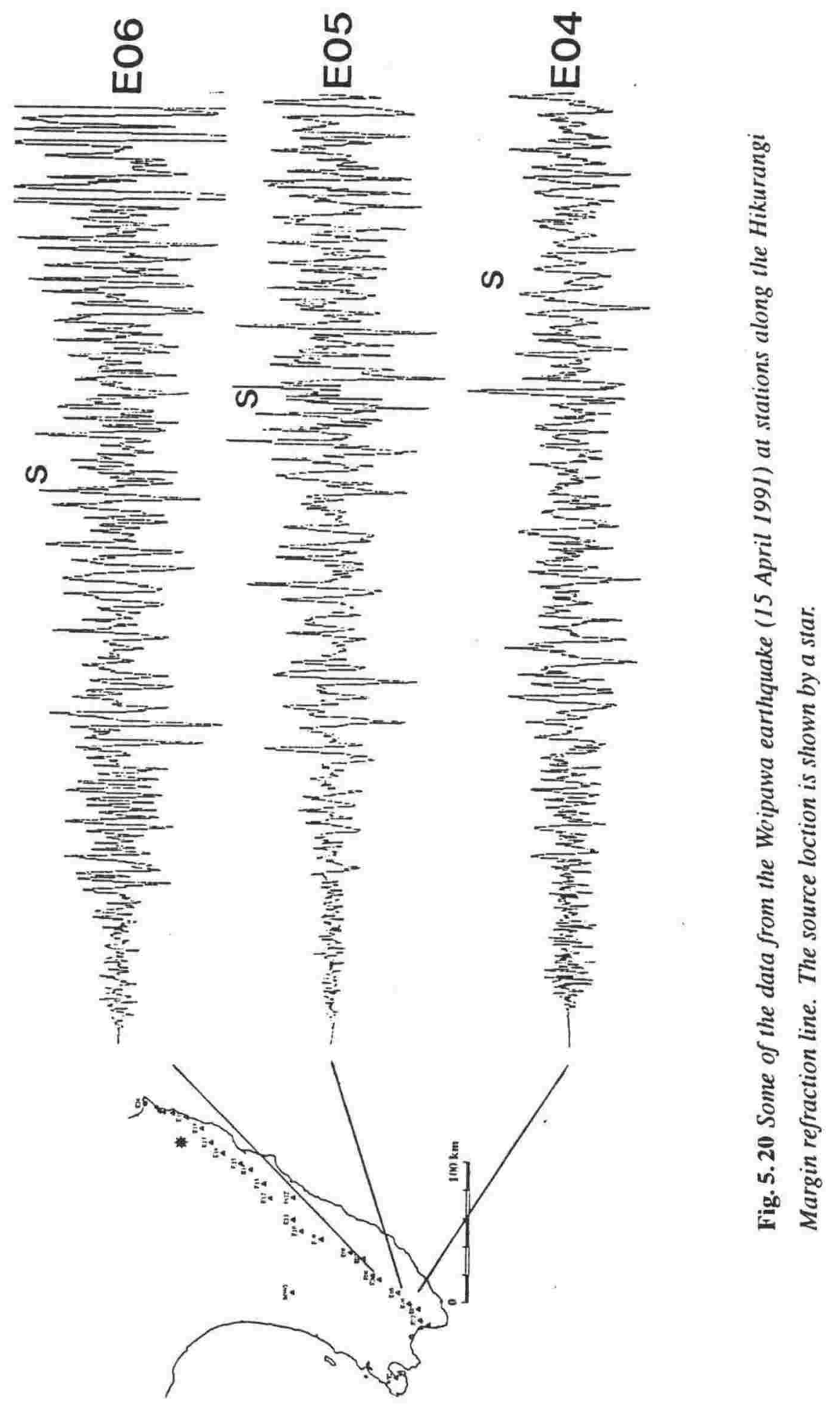


Figures 5.22a through $5.22 \mathrm{e}$, this velocity is increased in steps of $0.4 \mathrm{~km} / \mathrm{s}$ from $4.0 \mathrm{~km} / \mathrm{s}$ to 5.6 $\mathrm{km} / \mathrm{s}$. Figure $5.22 \mathrm{f}$ is the seismogram from MOD01, i.e. with no low-velocity layer. The wave velocities in the sediment layer appear to be critical in shaping different parts of the seismogram. In Figure 5.22e, in which the velocity contrast between the layer and the surrounding media is least, the reverberation characteristics approach those of the seismogram from MOD01 (Figure $22 \mathrm{f}$ ), while a P-velocity of around $4.8 \mathrm{~km} / \mathrm{s}$ within the layer produces reverberations most similar to those observed along the East Coast.

\subsubsection{Conclusions}

This simple modelling shows that, given enough data, it might be possible to draw conclusions about the existence and nature of a low-velocity layer on top of the subducted crust. By simple visual comparison of the synthetic seismograms with the East Coast data shown in Section 5.7.1, it could be tentatively suggested that the difference in character between the waveforms for wavepaths along the East Coast and those on moving further inland may well be explained by the presence or absence of a layer of inverted P-velocity at the top of the subducted slab. From the synthetic data, a 1 - $3 \mathrm{~km}$ thick layer, with P-velocity of $4.8 \pm 0.4 \mathrm{~km} / \mathrm{s}$ appears to yield waveforms closest in character to those observed in the limited data available in this study. The presence of this layer would confirm previous reports of its existence along the East Coast, but the waveform modelling results may also imply the thinning out, or compaction, of the subducted sedimentary material as the plate bends down.

Another effect that would be worth studying is the effect of curvature of the slab surface on the development of the reverberatory wavetrain. The modelling here has necessarily been confined to a plane slab surface, which is only approximately true in the region under consideration. Figure 5.17c suggests that a large contribution to the dominant wavetrain comes from below the slab surface, and therefore a reason for the absence of this wavetrain from the Weber data set (which represents an off-strike wavepath) might be the interference of the curvature, albeit slight, with the waveguide effect. On the other hand, if the interpretation of $\bar{P}$ in the Weber data set as a crustal waveguide effect is correct, then this does not seem to be adversely affected by the curvature. This may be because this wave group is more related to reverberations within the overlying crust itself.

\subsection{Effects of other parameters on the synthetic seismogram}

The preceeding sections were intended to examine, in some detail, the effects of particular features of interest within the velocity model for the subducted lithosphere, and to attempt to establish the presence or absence, and the nature of such features. In the previous models, the other parameters of the velocity and source model, such as crustal velocities, nature of the source 
and attenuation parameters were kept fixed. It is important to assess the effect of such other features on the seismogram so that we can be certain of the reality of the conclusions obtained above.

The number of parameters involved in the whole model is so large as to make it impractical to explore the full variation of each parameter. In the following sections, therefore, only a few key features are investigated, and this is done within the bounds of reasonable estimates of these features and within the bounds established by the results of previous workers. Fortunately, the parameters which have so far been kept constant have a minimal effect on the nature of the seismogram.

\subsubsection{Source depth, for a source within the overlying crust}

Robinson (1994), from travel time inversion, obtains hypocentres in the depth range 5 to 18 $\mathrm{km}$ for the 13 May 1990 aftershocks. Keeping the focal mechanism constant, and the source within the overlying crust, the synthetic seismogram for MOD01 was computed with the source at depths of $8,12,14$ and $16 \mathrm{~km}$. This places the $8 \mathrm{~km}$ deep source within the $5.5 \mathrm{~km} / \mathrm{s}$ layer, and the rest of the sources within the $6.2 \mathrm{~km} / \mathrm{s}$ layer. The seismograms are shown in Figure 5.23. There are small differences in the arrival times of the major phases, as well as in the details of the $\bar{P}$ group, but such variation is of course observed to a much higher degree in the real data.

\subsubsection{Focal mechanism, for a source within the overlying crust}

Also following Robinson's conclusions, a thrust type focal mechanism has been assumed for the source, with the parameters as given in Section 5.4. The effect of variations in fault plane and slip parameters was also explored. Figure 5.24 shows some synthetic seismograms for source models whose parameters vary within a small range around those of the basic source model. The source depth is $12 \mathrm{~km}$ in all cases. The fault plane parameters are printed beside each seismogram. The differences in the seismograms are negligible, the main change being in the shape of the direct wave pulse, arriving at reduced time about $7 \mathrm{~s}$.

\subsubsection{Crustal velocity model}

The presence of a low velocity layer at the free surface, representing weathered material, has already been discussed in Section 5.5. Its effect on the synthetic seismogram was to slightly increase the efficiency of the reverberatory $\bar{P}$ phase in the crustal waveguide.

The values of $5.5 \mathrm{~km} / \mathrm{s}$ and $6.2 \mathrm{~km} / \mathrm{s}$ for the 2 main crustal layers are consistent with velocity models for the crust in the North Island arrived at by previous workers (Figure 5.5) and may be regarded as an average profile for these models. The presence of velocity gradients within these layers was briefly investigated and found to make no significant difference to the synthetic waveforms. Figure 5.25 shows the seismogram for the basic model MOD01, and that from a velocity profile in which the $5.5 \mathrm{~km} / \mathrm{s}$ layer is replaced by a linear gradient of $0.0824 / \mathrm{s}$. The 


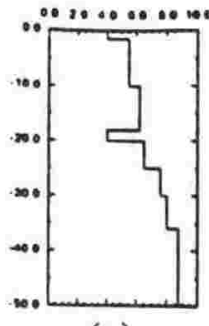

(a)

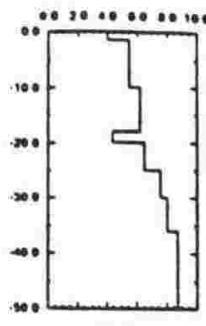

(b)

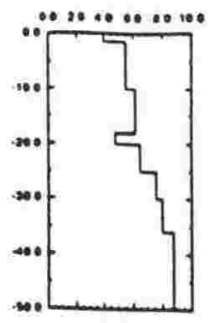

(c)

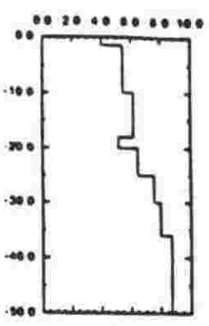

(d)

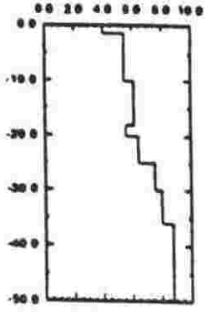

(e)

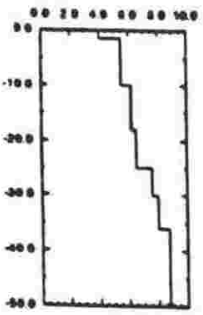

(f)

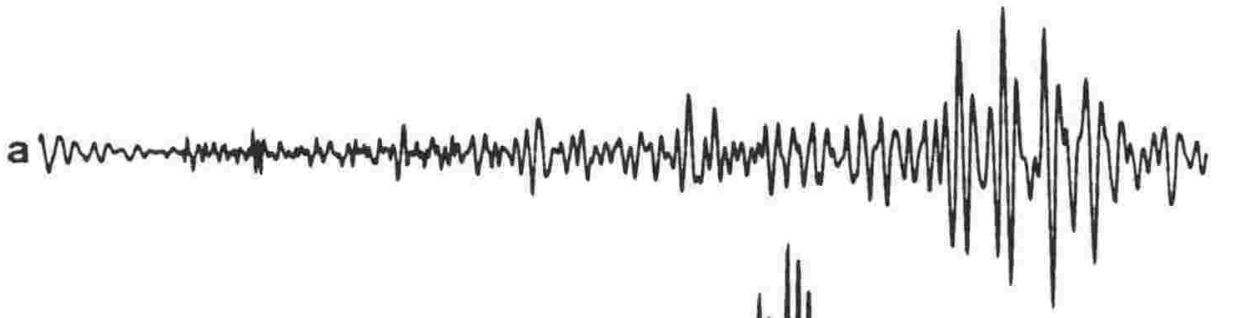

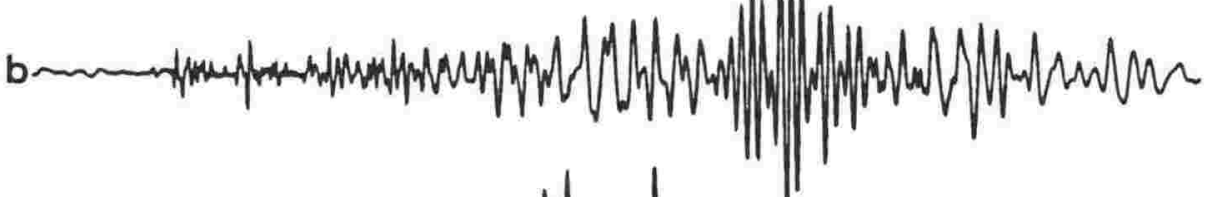

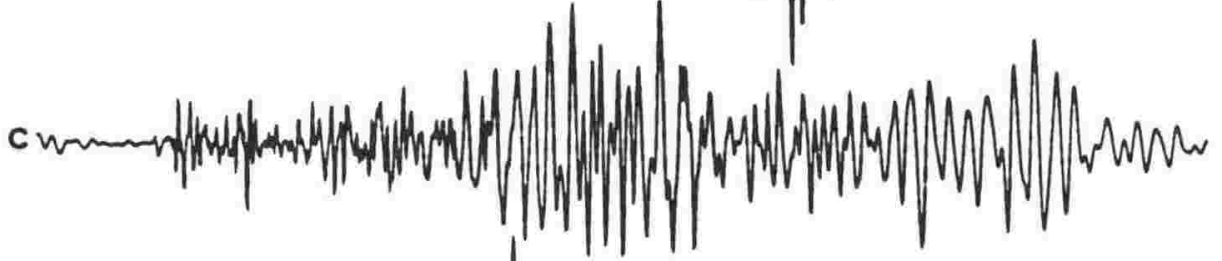

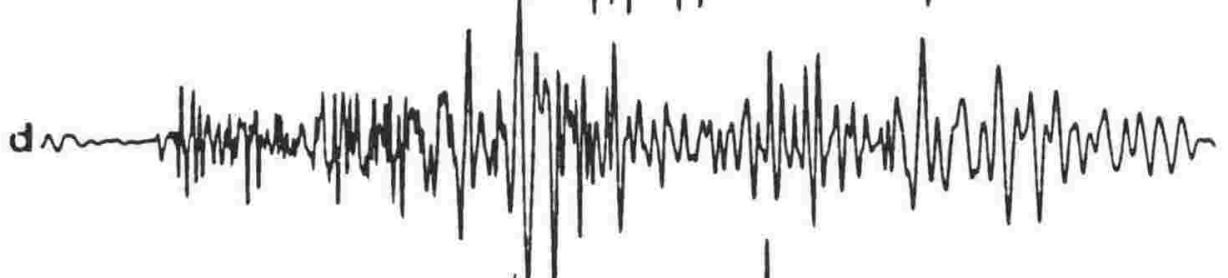

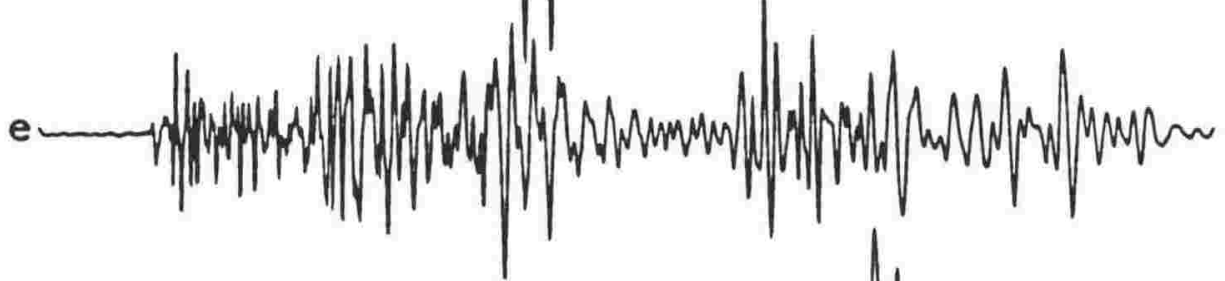

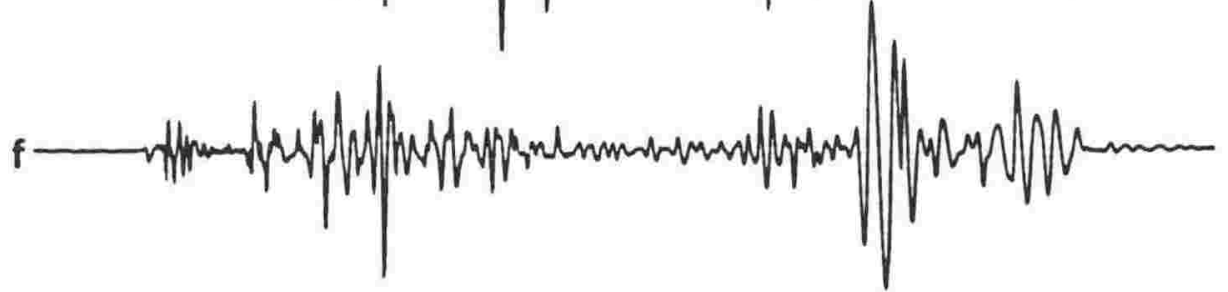

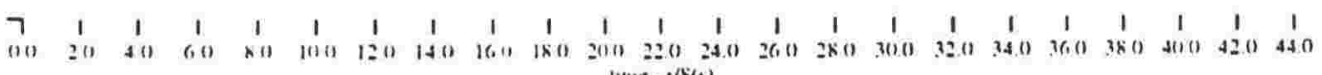

Fig. 5.22 Effect of varying the P-velocity within a $2 \mathrm{~km}$-thick, subducted low-velocity layer a) $4.0 \mathrm{~km} / \mathrm{s}$ b) $4.4 \mathrm{~km} / \mathrm{s}$ c) $4.8 \mathrm{~km} / \mathrm{s}$ d) $5.2 \mathrm{~km} / \mathrm{s}$ e) $5.6 \mathrm{~km} / \mathrm{s}$. Trace f is from $M O D O 1$, with no low velocity layer. 


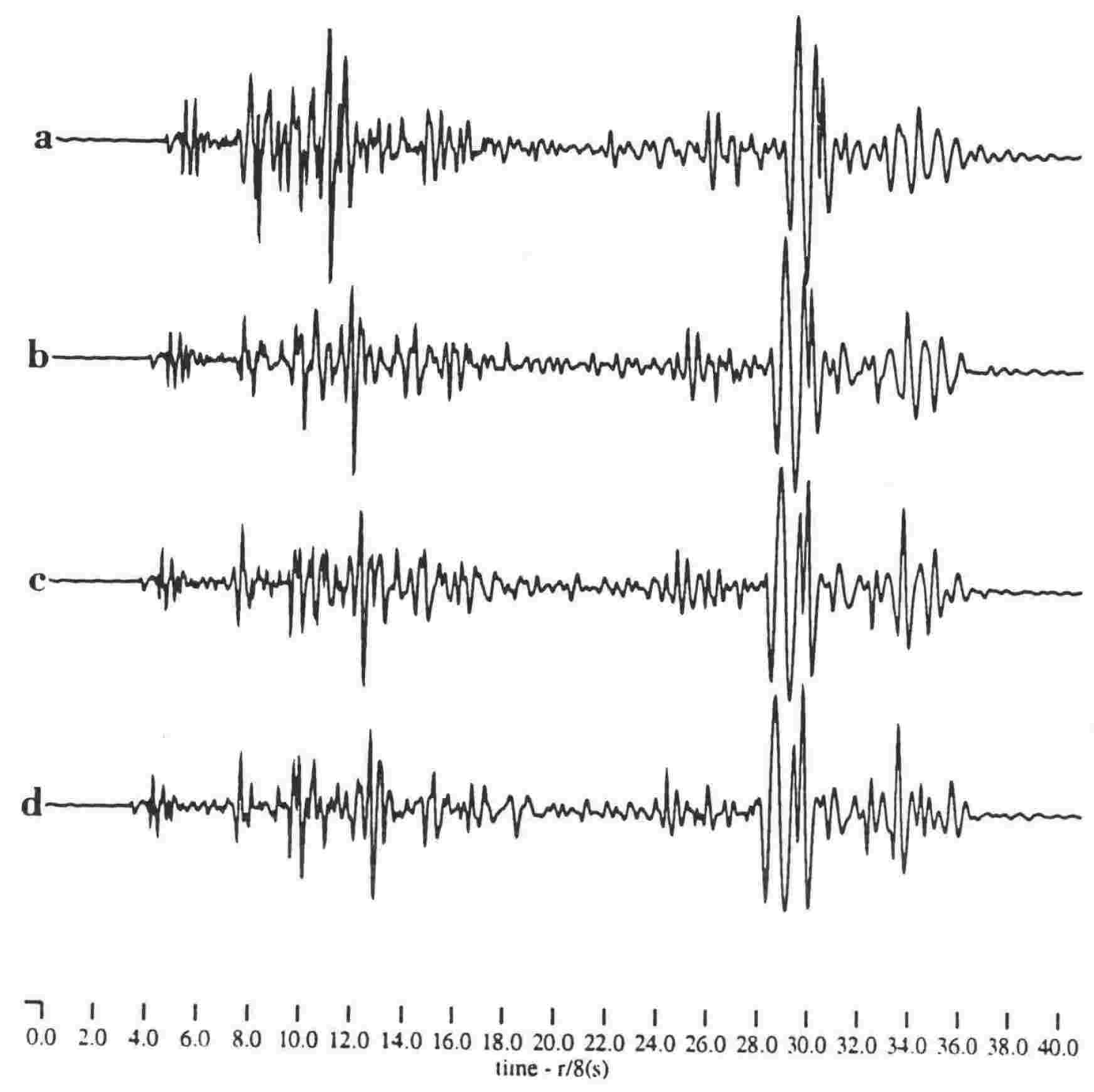

Fig.5.23 Effect of source depth for a source within the overlying crust, having the same mechanism as has been used thus far with model MODO1. a) $8 \mathrm{~km} \mathrm{b)} 12 \mathrm{~km} \mathrm{c)} 14 \mathrm{~km} \mathrm{d)} 16 \mathrm{~km}$ deep.

difference in the seismograms is insignificant.

Another crustal feature which was investigated was the presence of a high velocity basal crustal layer. Such a layer, of P-velocity $6.7 \mathrm{~km} / \mathrm{s}$, is present in Chong's (1982) Hawkes Bay model, and was also modelled in Chapter 3 to fit the apparent velocity of the first arrival by means of 3-dimensional ray-tracing. The velocity model for such a profile is shown in Figure 5.26, again with that from MOD01 for comparison. As expected, the presence of a high-velocity layer at the base of the crust did affect the nature of the $\bar{P}$ phase, producing a wave group of a lower frequency and a more oscillatory nature than that from MOD01. It is difficult to conclude 


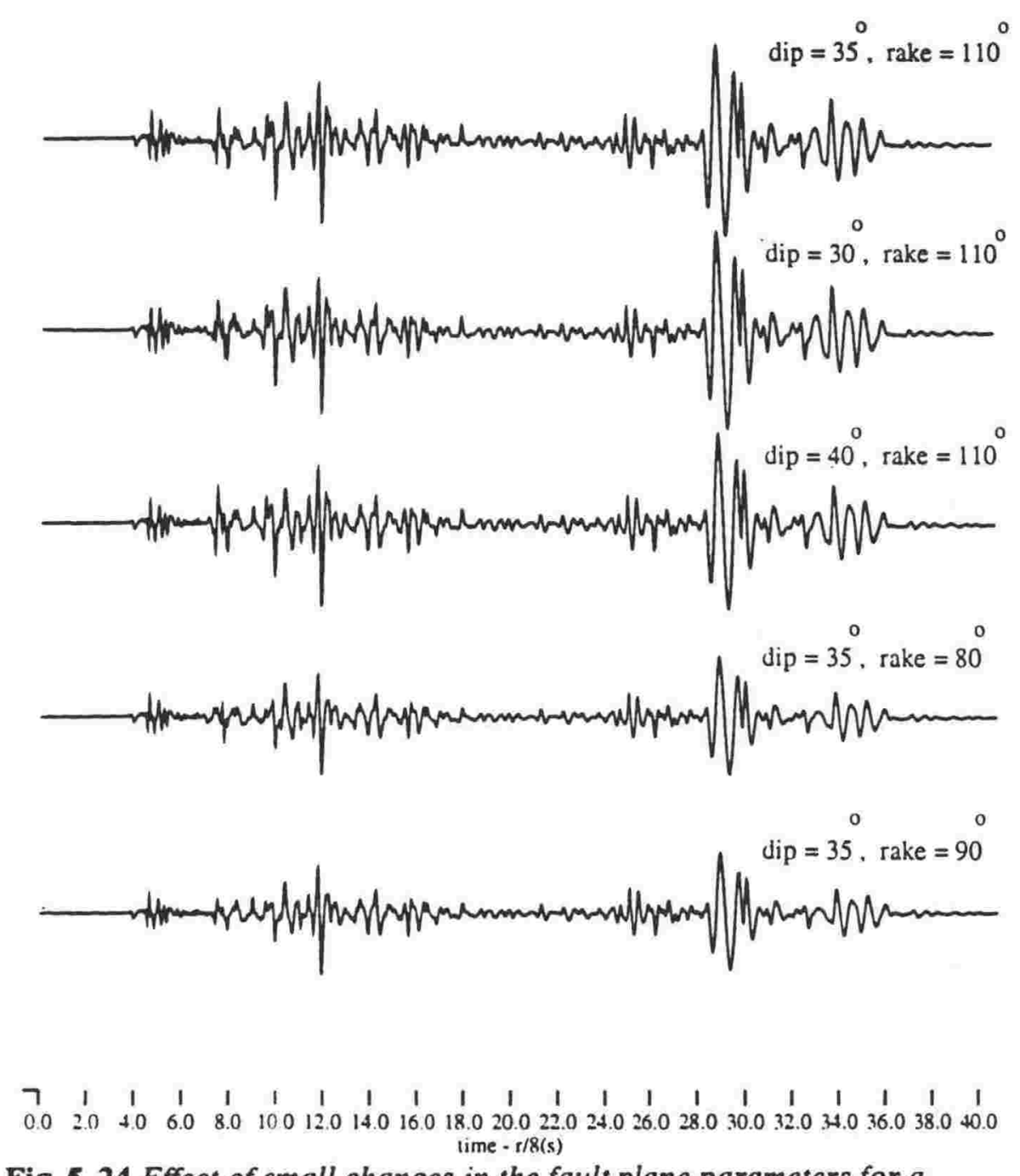

Fig. 5.24 Effect of small changes in the fault plane parameters for a source at $12 \mathrm{~km}$ depth, and using model MOD01.

The parameters are printed beside each trace.

whether such a waveform is more, or less, similar to the real data (Figure 5.1) However, it can be stated that the presence of such a layer is not incompatible with the observations.

\subsubsection{Attenuation}

Modelling the $\mathrm{P}$ and $\mathrm{S}$ attenuation parameters within each layer of the velocity model would involve a time-consuming study on its own since the combination of a large range of parameters would need to be investigated. Also the available data do not allow the investigation of variation of signal amplitude with epicentral distance. This will be better controlled with data such as that from the Hikurangi Margin refraction experiment.

The extent of attenuation modelling carried out in this section was the division of the whole profile into 3 regions - the surface weathered layer, the overlying and subducted crust, and the subducted upper mantle. The term "attenuation parameter", which may also be called the loss factor, refers to the quantity $Q^{-1}$, where $Q$ is the quality factor (see Chapter 4 , Section 4.10 ). 


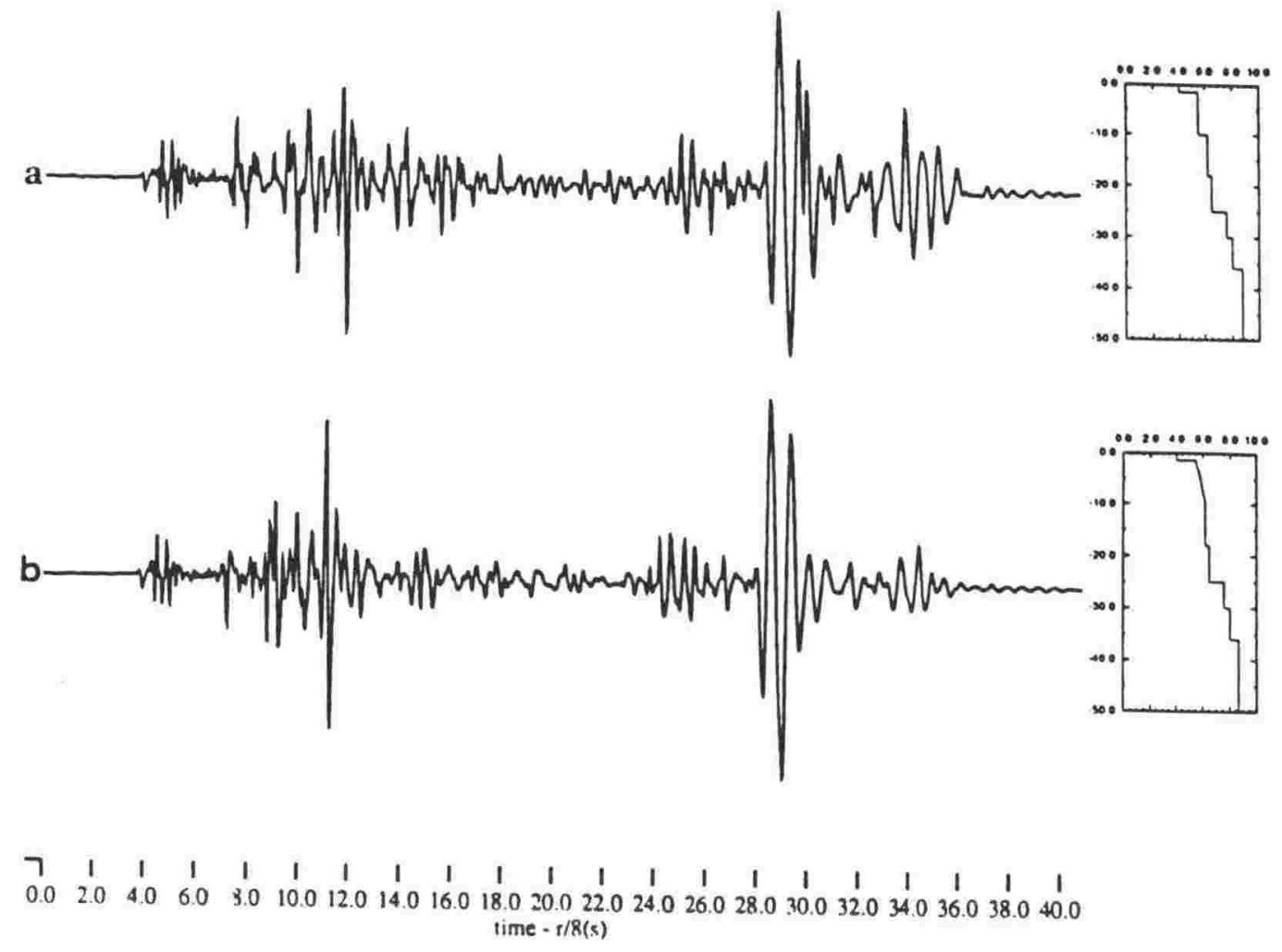

Fig. 5.25 Effect of replacing the $5.5 \mathrm{~km} / \mathrm{s}$ crustal layer by one having a linear velocity gradient. The top trace is from MODO1.

Table 5.2 lists the attenuation models examined for profile MOD01, while Figure 5.27 shows the corresponding synthetic seismograms. It is seen from these seismograms that the major effects of the attenuation parameters are two: the amplitude of the S-wave train relative to that of the Pwave train, and the persistence of the high-frequency content within the P-wave train. Again, it is difficult to arrive at a conclusion about the best attenuation model on the basis of comparison with this particular data set. The model with very low attenuation $\left(\mathrm{Q}_{\mathrm{P}}^{-1}=0.001, \mathrm{Q}_{\mathrm{S}}^{-1}=0.002\right)$ in all layers should be discarded as it leads to too high S-wave amplitudes, while that with the highest attenuation leads to the elimination of the high-frequency content. A model in which the crustal material has an average attenuation parameter of $Q_{P}^{-1}=0.002$ and $Q_{S}^{-1}=0.004$ $\left(Q_{P}=500, Q_{S}=250\right)$ with the lower mantle possessing very low attenuation appears to be a reasonably satisfactory working model. Chadwick (1991), in a source parameter/attenuation study for the Wellington region, arrives at an average value of $Q_{S}=568 \pm 70$ for the crustal material and $1316 \pm 150$ for the upper mantle material. The approach used here cannot be regarded as a reliable method for modelling attenuation parameters, but only as a rough means of 
establishing some reasonable bounds. The high-frequency content of the first few seconds of the observed data, however, corresponding to waves propagating mainly within the subducted upper mantle, do suggest the presence of low attenuation material at that depth, and this is not inconsistent with the results of waveform modelling.

\subsubsection{Events within the subducted lithosphere}

As discussed in Chapter 2, the aftershocks of the May 13 event, occurring within the overlying crust were contemporaneous with the ongoing aftershock sequence of the 19 February mainshock, which occurred below the subduction interface. According to Robinson, the latter aftershocks occurred in the depth range $22-35 \mathrm{~km}$, and their focal mechanism was averaged by a normal faulting process on a steeper, $75^{\circ}$ dipping fault plane. The synthetic seismograms resulting from this kind of source were computed so that the effect of this parameter could also be evaluated. For such a representative source, the following parameters were chosen:

$$
\phi_{f}=170^{\circ} ; \delta=75^{\circ} ; \lambda=270^{\circ} ; \phi=190^{\circ}
$$

giving a moment tensor of

$$
\left(\begin{array}{ccc}
0.015 & 0.086 & -0.150 \\
0.086 & -0.485 & -0.853 \\
-0.150 & -0.853 & -0.500
\end{array}\right)
$$

A source of this type was placed at three different depths within the subducted lithosphere $(22,26$ and $32 \mathrm{~km}$ ), using the same velocity model MOD01, resulting in the synthetic seismograms in Figure 5.28. It is seen that the nature of the seismograms is quite different from those due to a thrust-type source above the subduction interface, for example Figure 5.23, and also quite

Table5.2 Different attenuation models tested. The attenuation parameter for $S$-waves, $\mathrm{Q}_{\mathrm{S}}^{-1}$ was chosen at twice that for $P$-waves, $\mathrm{Q}_{\mathrm{P}}^{-1}$. The 3 layers in which $\mathrm{Q}_{\mathrm{P}}^{-1}$ and $\mathrm{Q}_{\mathrm{S}}^{-1}$ are defined refer respectively to (i) the surface weathered layer

(ii) the overlying crust together with the subducted crust down to the $7.6 \mathrm{~km} / \mathrm{s}$ layer, and

\begin{tabular}{|c|c|c|c|c|c|c|c|}
\hline $\begin{array}{c}\text { MOD01.a0 } \\
\mathrm{Q}_{\mathrm{p}}^{-1}\end{array}$ & $Q_{S}^{-1}$ & $\begin{array}{c}\text { MOD01.al } \\
\mathrm{Q}_{\mathrm{p}}^{-1}\end{array}$ & $\mathrm{Q}_{\mathrm{S}}^{-1}$ & $\begin{array}{c}\text { MOD01.a2 } \\
\mathrm{Q}_{\mathrm{p}}^{-1}\end{array}$ & $Q_{S}^{-1}$ & $\begin{array}{c}\mathrm{MOD} 01 . \mathrm{a3} \\
\mathrm{Q}_{\mathrm{p}}^{-1}\end{array}$ & $\mathrm{Q}_{\mathrm{s}}^{-1}$ \\
\hline 0.001 & 0.002 & 0.01 & 0.02 & 0.01 & 0.02 & 0.01 & 0.02 \\
\hline 0.001 & 0.002 & 0.001 & 0.002 & 0.002 & 0.004 & 0.004 & 0.008 \\
\hline 0.001 & 0.002 & 0.001 & 0.002 & 0.001 & 0.002 & 0.001 & 0.002 \\
\hline
\end{tabular}
(iii) the subducted upper mantle 

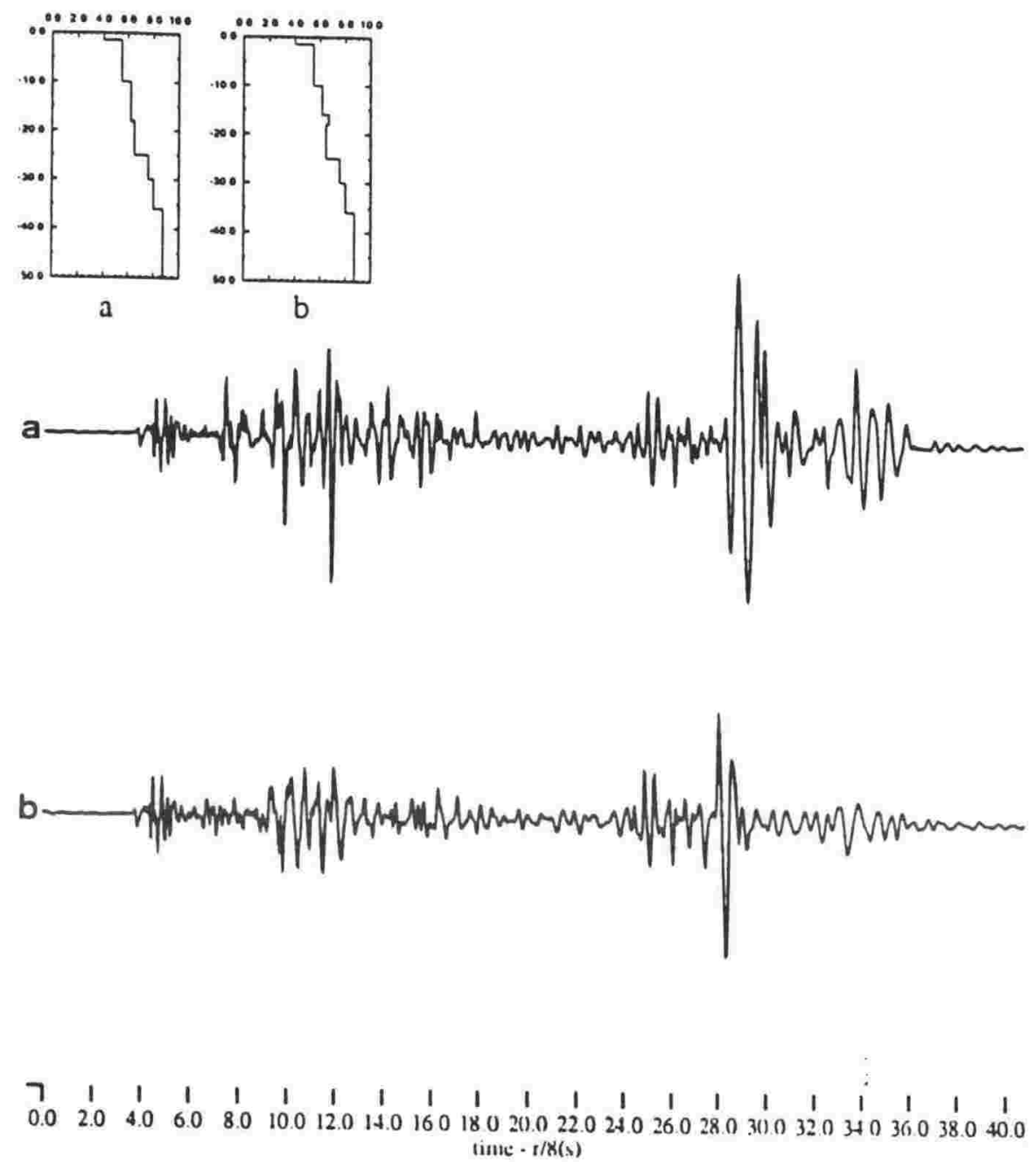

Fig. 5.26 Effect of introducing a high-velocity $(6.7 \mathrm{~km} / \mathrm{s})$ basal layer in the overlying crust, compared with the seismogram from MODOI. 


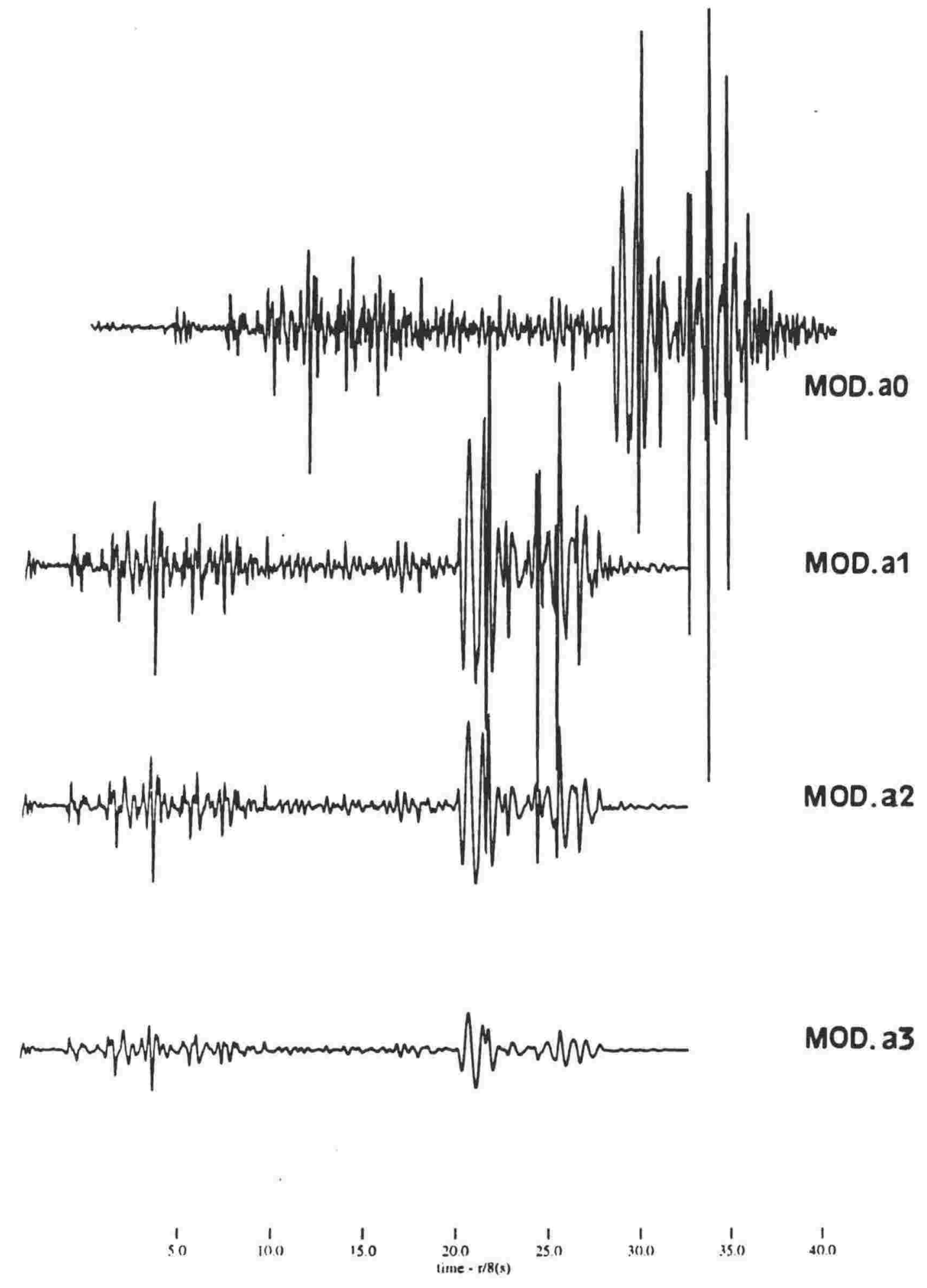

Fig. 5.27 Effect of varying the attenuation parameter in the different regions of the lithosphere. The actual values of the attenuation parameter are listed in Table 5.2. The first trace is displaced for clarity. 
different from the observed data. This suggests that the data set studied here represents aftershocks from the 13 May event only. This is reasonable in view of the larger incidence of these shocks on the 13 May 1990, and the ensuing few days.
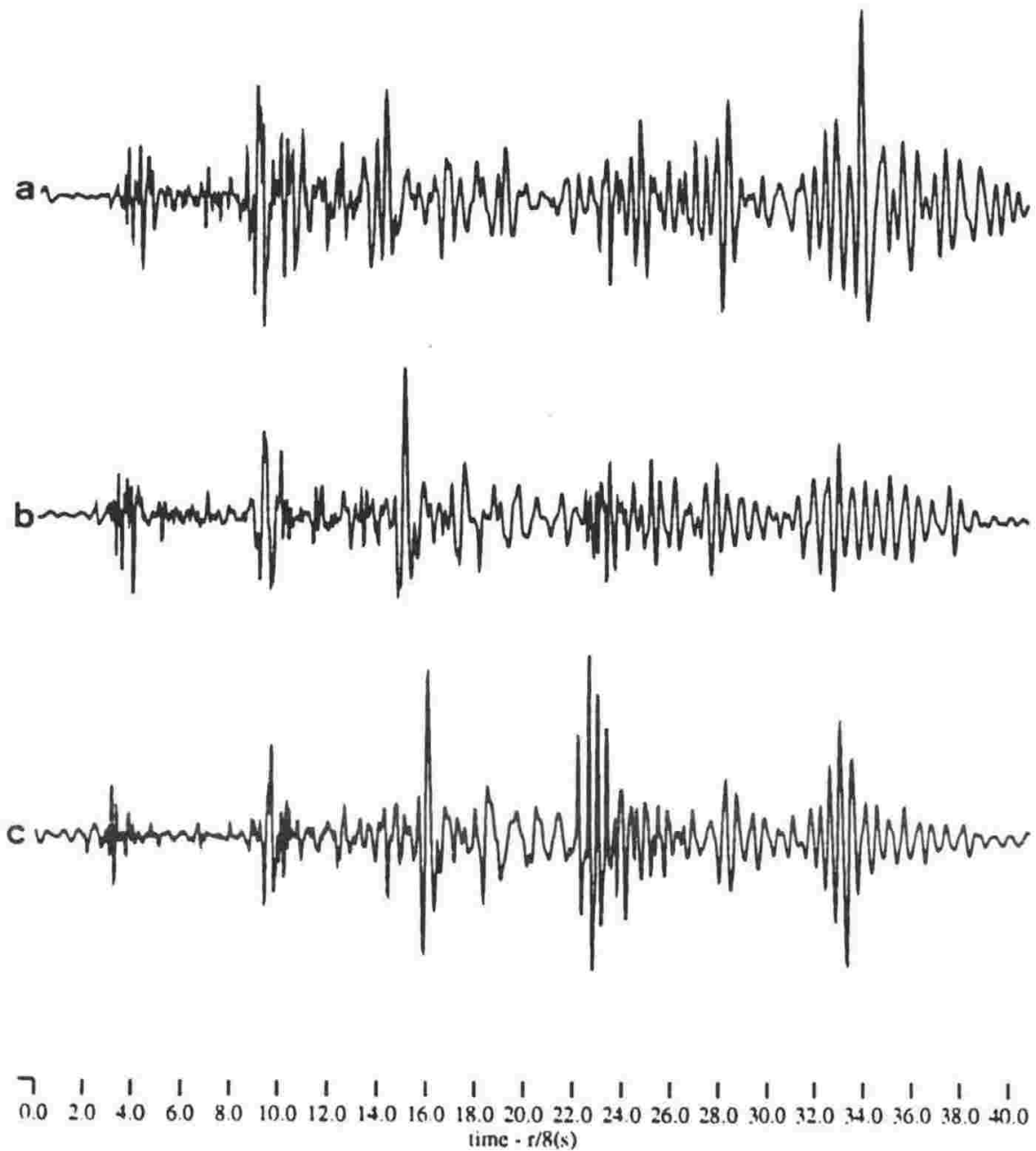

Fig. 5.28 Synthetic seismograms for a normal faulting source within the subducted lithosphere at depths of a)22 km b) $26 \mathrm{~km}$ and c) $32 \mathrm{~km}$. The velocity model is MODO1. 


\subsection{Comparison with 3-dimensional ray-tracing results}

In Chapter 3, a working velocity model (Figure 3.9) was derived on the basis of fitting the apparent speed, azimuth and travel time of the first arrival through a 3-d structure, using complete ray-tracing. It is now intended to reassess the velocity model found in the present chapter, by adapting it to a 3-dimensional structure, having the subducted slab curvature discussed in Chapter 3. Such a velocity profile will have a high-velocity basal layer in the overlying crust, because, although this layer is not strictly required by the waveform modelling process, it was found to explain better the apparent speeds and incident azimuths. A cross-section of the 3-d model is shown in Figure 5.29, with the source placed at $12 \mathrm{~km}$ depth.

Keeping the source and receiver positions identical to those used in Chapter 3 (Section 3.4.2), the CRT program yielded the following values for travel time and apparent velocity at the station for a first arriving phase propagating through the $8.75 \mathrm{~km} / \mathrm{s}$ layer:

$$
t=25.48 \mathrm{~s} ; c=8.61 \mathrm{~km} / \mathrm{s} ; \phi=20.15^{\circ}
$$

This is in good agreement with the observed average values of $25.5 \mathrm{~s}, 8.7 \mathrm{~km} / \mathrm{s}$ and $16^{\circ}$ obtained from the real data in Chapter 2.

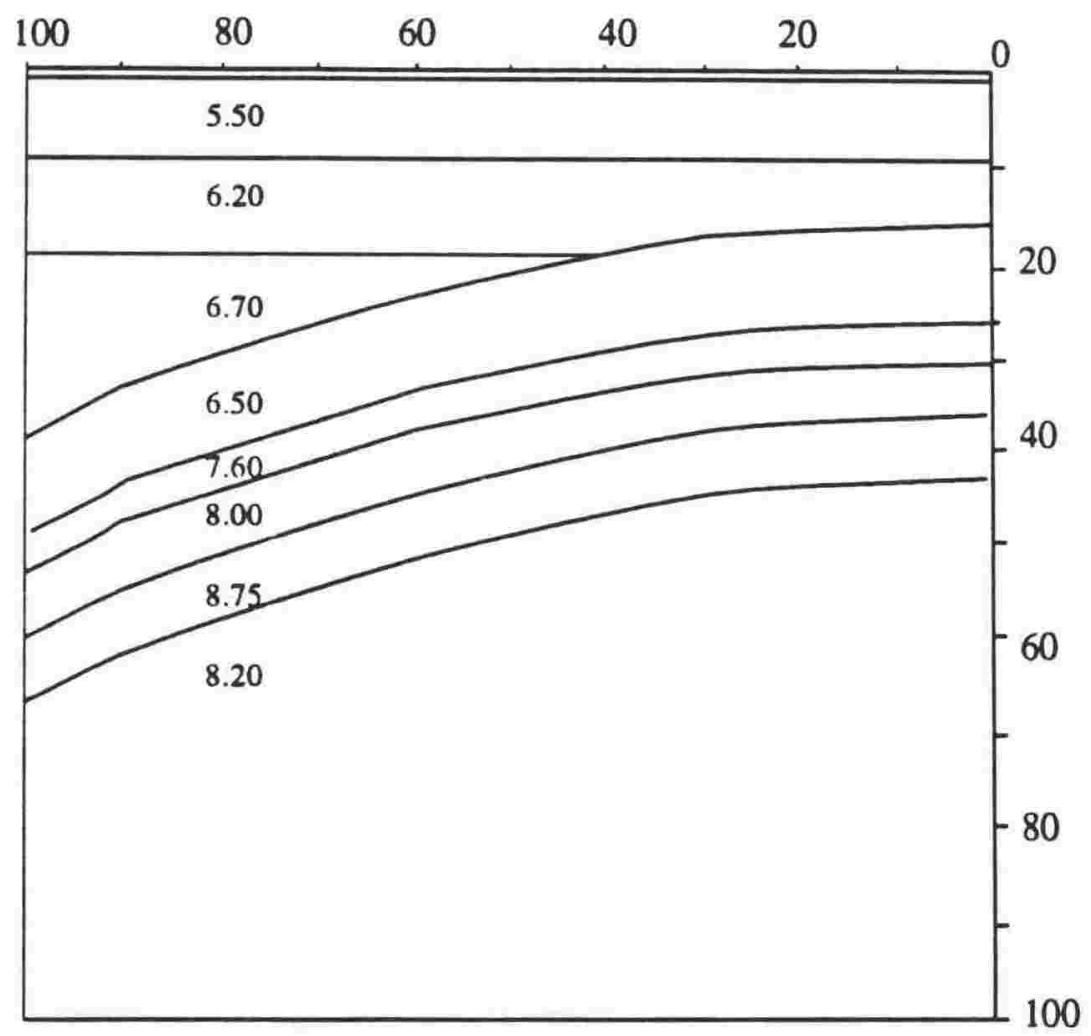

Fig. 5.29 Cross section of 3-d model incorporating velocity features found by waveform modelling and having the curvature used in Chapter 3. This model is now used to re-estimate the theoretical apparent velocity of the first-arriving head wave. 


\subsection{Conclusion}

A velocity profile can finally be adopted which satisfies the requirements of both the 3-dimensional ray-tracing and of the waveform modelling techniques. This profile is shown as a 1-dimensional approximation in Figure 5.30. This model is to be regarded as an average structure

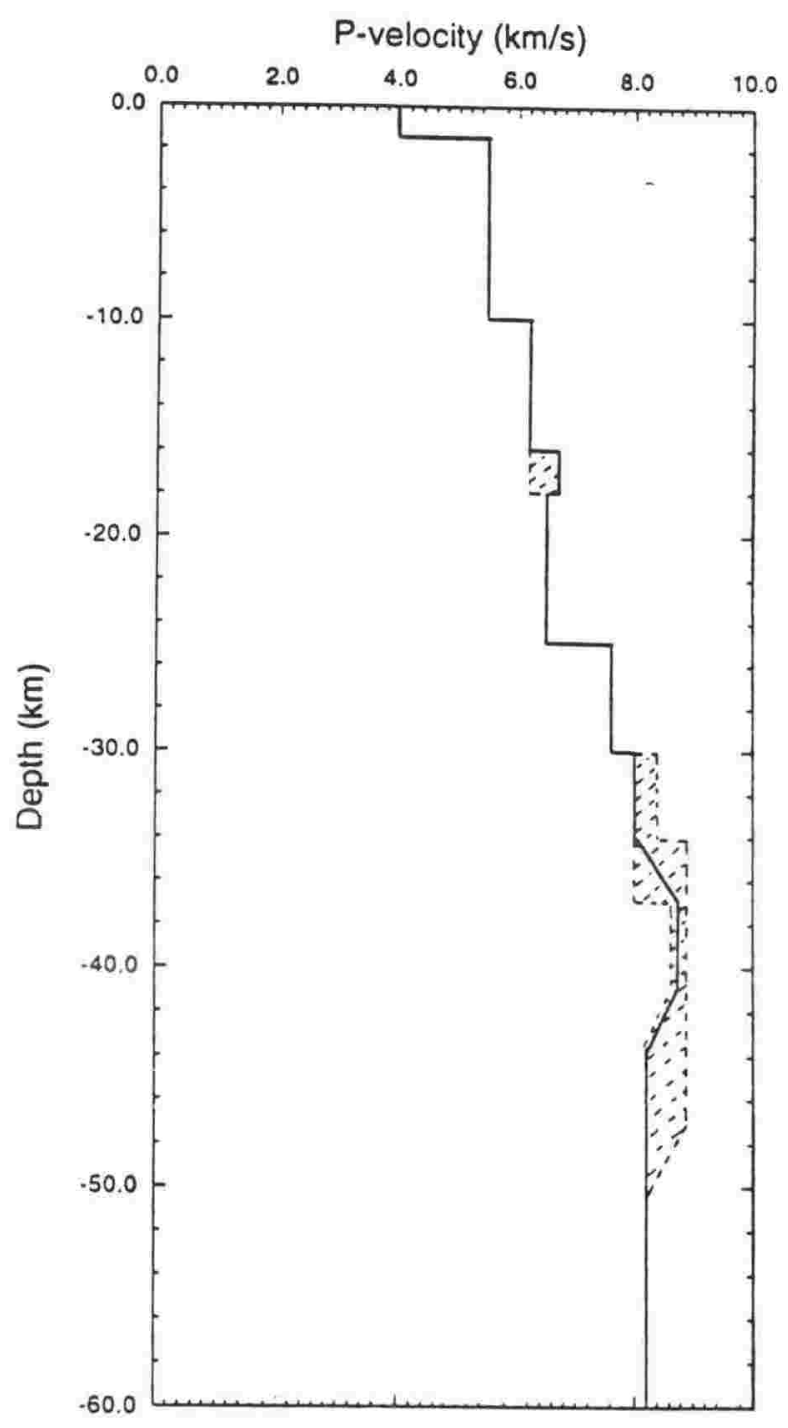

Fig. 5.30 A final velocity profile satisfying the requirements of both 3-dimensional ray tracing as well as waveform modelling. Limits of velocity constraints for layers examined in this chapter shown by shaded regions. 
for the south-eastern part of the North Island. Where some constraint on parameters could be made through the modelling processes, an indication of the limits of these constraints is given by the shading on the diagram. Features such as the crustal velocity structure and the P-velocity below the high- velocity medium, which were not fully investigated in this study are not to be regarded as uniquely determined but as the fixed values used in the basic model. The velocity gradients are also quite arbitrary.

Figure 5.31 shows the velocity profile for the case of a low-velocity layer at the top of the subducted lithosphere, which model might represent the structure further towards the East Coast.

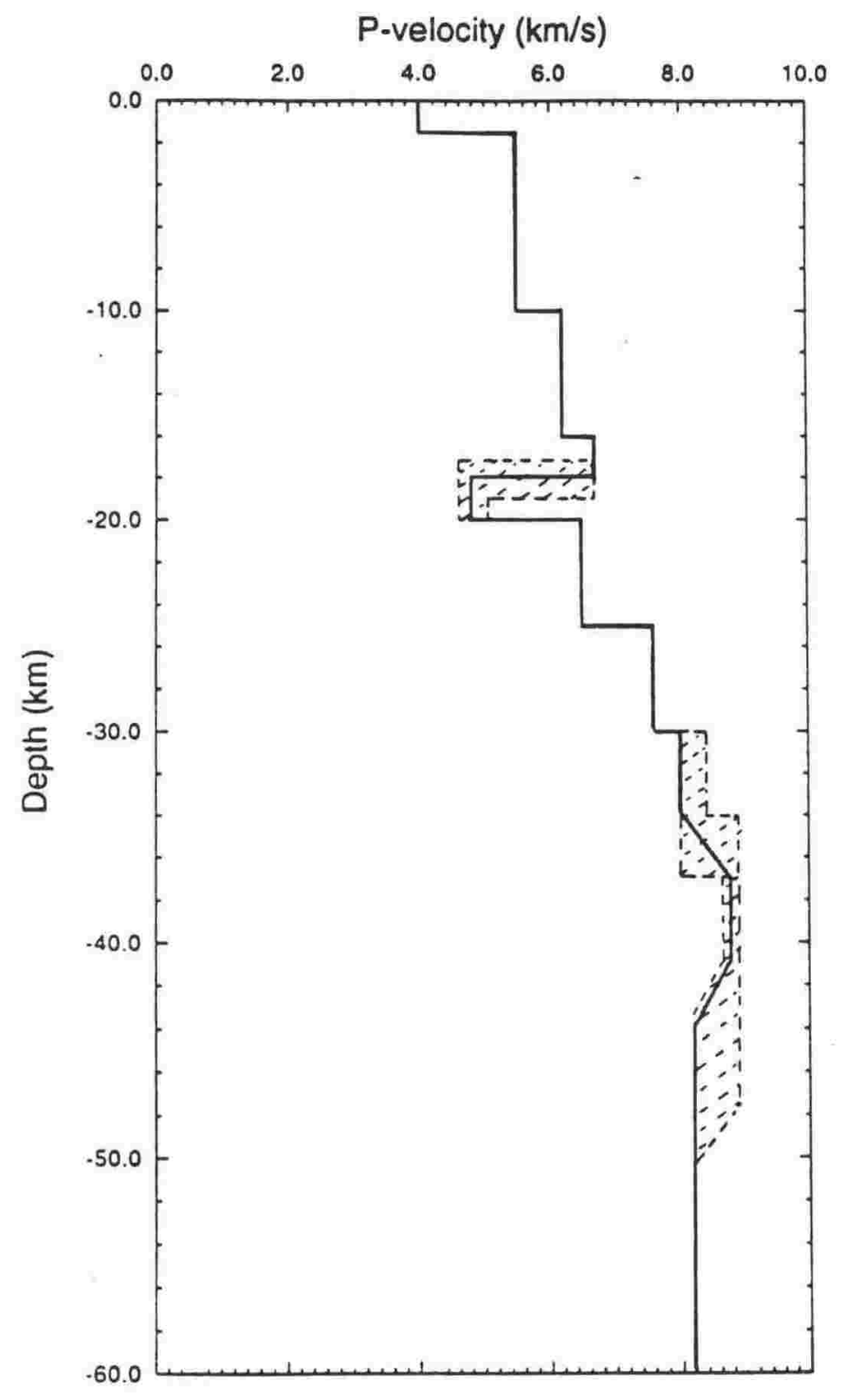

Fig. 5.31 Velocity profile for model with a low-velocity subducted layer. 


\section{Chapter 6}

\section{Upper Mantle Anisotropy in the South West Pacific Ocean}

\subsection{Introduction}

Velocities of the order of $8.75 \mathrm{~km} / \mathrm{s}$, such as those discussed in the previous chapters are not typical of average oceanic upper mantle materials. Similarly high P-velocities have, however, been reported in many cases, both "in situ" in oceanic lithosphere (e. $g$ Hales et al, 1970; Sverev and Yaroshevskay, 1987) as well as in ophiolitic rocks (e.g Christensen and Ramananantoandro, 1971; Baker and Carter, 1972; Boudier and Nicolas, 1985) which are relics of oceanic crust thrust up to a continental surface. These occurrences will be more fully discussed in the next chapter. It is, however, necessary here to say that practically all such high velocities are associated with anisotropic media, and more specifically with the anisotropy of olivine, which is a major constituent of the oceanic upper mantle. Single-crystal olivine is known to possess a high degree of P-wave anisotropy with the compressional velocity varying between $7.72 \mathrm{~km} / \mathrm{s}$ along the slow axis to $9.89 \mathrm{~km} / \mathrm{s}$ along a perpendicular axis (Verma, 1960). The anisotropy of olivine-rich upper mantle materials derives from the process whereby olivine crystals align thernselves with their fast axes parallel to the spreading direction during the formation of new ocean floor at a midocean ridge (Fuchs, 1977).

The geometry of a subducting plate makes it difficult to investigate the azimuthal variation of $P_{n}$ velocity withun it. It was thought, however, that it might be useful to look for such a variation within the stable Pacific plate before it begins to subduct below the Tonga-Kermadec trench and below the North Island, New Zealand. The only direct investigation of upper mantle P-wave anisotropy in this area was conducted by Shearer and Orcutt (1985) who set up a star-shaped set of four refraction profiles, about $100 \mathrm{~km}$ long, at a point approximately $1000 \mathrm{~km}$ east of the Tonga trench and $1500 \mathrm{~km}$ WSW of Tahiti. The experiment was conducted in oceanic lithosphere believed to be about $140 \mathrm{My}$ old, one of the oldest areas in the Pacific. Their results provided evidence for upper mantle anisotropy, with the P-velocity varying between $8.5 \mathrm{~km} / \mathrm{s}$ along a direction $\mathrm{N} 30^{\circ} \mathrm{E}$ and $8.0 \mathrm{~km} / \mathrm{s}$ along a perpendicular axis. The authors infer from these results a fossil spreading direction of $\mathrm{N} 30^{\circ} \mathrm{E}$ for this part of the Pacific lithosphere.

A completely different approach to investigating mantle anisotropy was taken by Montagner and Tanimoto (1991), who used a global data set of Rayleigh and Love wave dispersion to invert for various seismic parameters (including anisotropy parameters) on a global scale. Their world map of S-wave azimuthal anisotropy calculated at $100 \mathrm{~km}$ depth shows a general $\mathrm{N}$-S direction 
for the fast axis in the south west Pacific.

In this study a direct approach has been taken, whereby P-wave travel times for a large number of seismic paths covering the area of interest were analysed for variation with azimuth. Hypocentre and arrival time data of earthquakes occurring along the Tonga-Kermadec trench and in the North Island, New Zealand, and recorded at stations NUE (Niue), RAR (Rarotonga) and CIZ (Chatham Islands) (Figure 6.1) were obtained from the I.S.C. bullettin for the period 1970 1987. The range of travel path directions covered enabled a distinct variation of P-wave velocity with azimuth of propagation to be detected, thus confirming the probable existence of large-scale upper mantle P-wave anisotropy in the Pacific plate.

\subsection{Data}

A study of the same region had been previously carried out by Prasad and Bock (1987) who analysed P-residuals (observed minus Jeffreys-Bullen predicted travel times) from events in the Tonga-Kermadec trench recorded at the stations NUE and RAR. They show clearly that, whereas earthquakes deeper than about $100 \mathrm{~km}$ produce $\mathrm{P}$-residuals very close to zero, and with a remarkably small scatter, shallow events produce negative P-residuals varying widely from 0 to $-16 \mathrm{~s}$. The authors used ray-tracing to model their results in terms of a high-velocity lid, of average P-velocity $8.3 \mathrm{~km} / \mathrm{s}$, in the uppermost mantle below the Pacific ocean crust. Whereas the first arriving waves from the deep earthquakes travel for most of their paths through the normal mantle, those from shallow earthquakes are mostly "guided" through the lid.

Prasad and Bock did not, however, attempt to explain the wide scatter in the residuals. In the present study, these authors' analysis is taken further by (a) using a larger data set, (b) using an extra recording station (CIZ) providing wider azimuthal coverage and (c) exploring whether there exists a systematic variation of the shallow earthquake residuals with azimuth of propagation.

Data was obtained from the I.S.C. Bulletin compiled on compact disc (ISC Bulletin Data Base contained on the Hypocenter Associated Data CD-ROM, USGS/NEIC)) for the period 1 January 1970 to 31 August 1987. The seismic region selected was the whole of the TongaKermadec trench system and the North Island of New Zealand (latitude range $14^{\circ} \mathrm{S}$ to $42^{\circ} \mathrm{S}$ ) and the recording stations were NUE, RAR and CIZ (Figure 6.1). The lower limit of $42^{\circ} \mathrm{S}$ ensured that propagation paths did not include the Chatham Rise and Campbell Plateau, which are believed to consist of crust type different from the Pacific ocean crust further north.

Figure 6.2 shows the variation of P-residuals (as given in the I.S.C. bullettin) versus source depth obtained in this study. The graph exhibits the same characteristics as those of Prasad and Bock's data set, namely that earthquakes deeper than about $100 \mathrm{~km}$ produce near-zero residuals at all stations, while the shallow events yield negative residuals with a high degree of variability. 


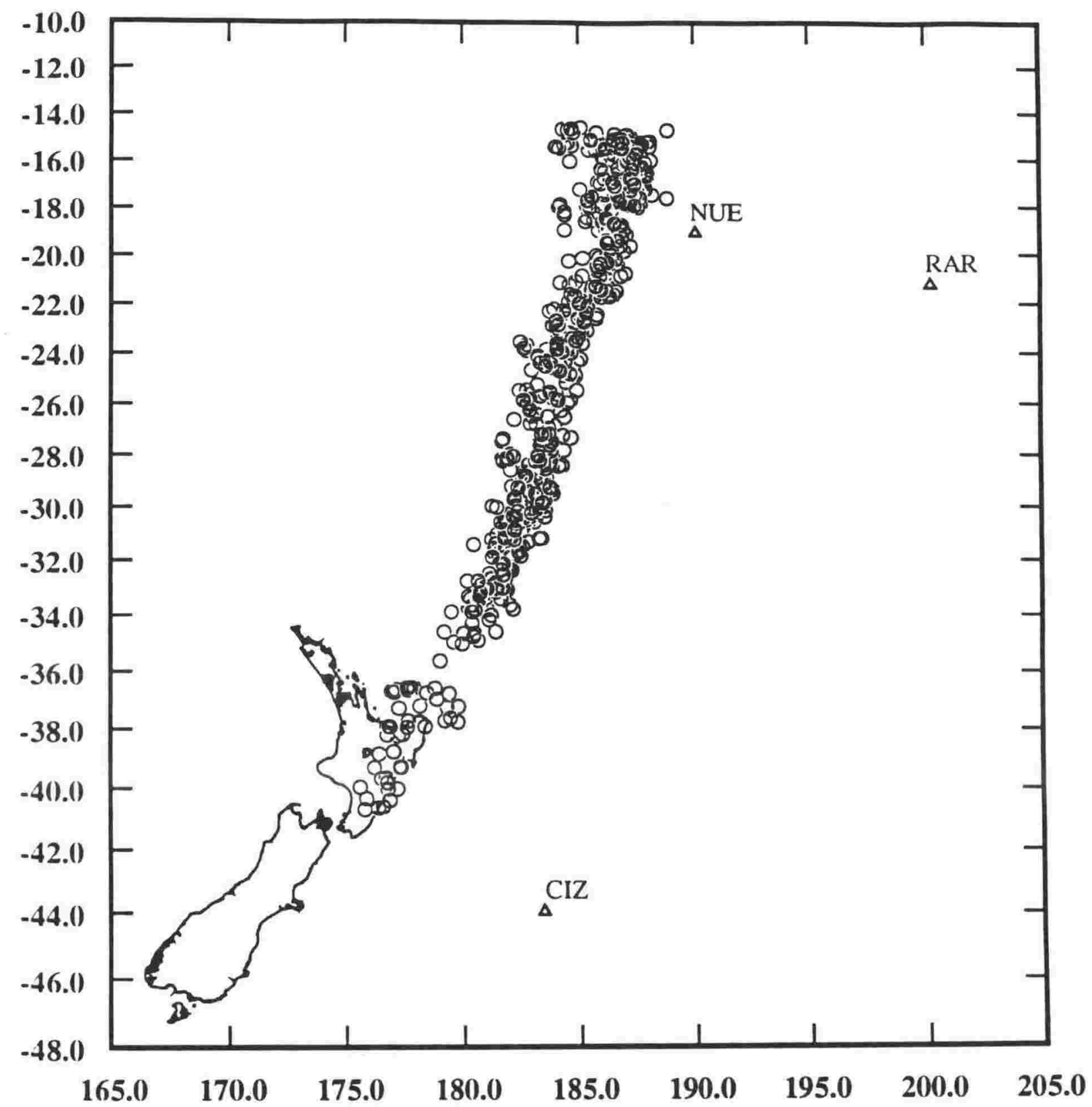

Fig. 6.1 Events and stations selected for this study. Epicentres shown are for shallow $(<100 \mathrm{~km})$ events. The period covered is 1 January 1970 to 31 August 1987. 
This plot justified the selection of only those events shallower than $100 \mathrm{~km}$ for investigation. No attempt was made at relocating the hypocentres, but events whose depths were constrained were not selected. In addition, so as to deal with the better located events, a further restriction was made by choosing earthquakes recorded at more than 100 stations worldwide. This number was, however, reduced to 50 in the case of events in the North Island, New Zealand, to compensate for the smaller number of large magnitude earthquakes in that region, and maintain a good coverage. The total number of events thus selected was 1187 . The body wave magnitudes of these events varied between 4.5 and 6.7 . Further checks with the I.S.C. bulletins showed that about $65 \%$ of the selected events had more accurate focal depth calculations based on pP-P intervals, and where these differed from those calculated only from $\mathrm{P}$ phases, the difference in focal depth rarely exceeded $50 \mathrm{~km}$, or placed the source much below the $100 \mathrm{~km}$ depth.

The azimuth and latitude ranges covered by the three stations is depicted in Figure 6.3. The azimuth is measured from epicentre to station, clockwise from North. For reasons to be discussed

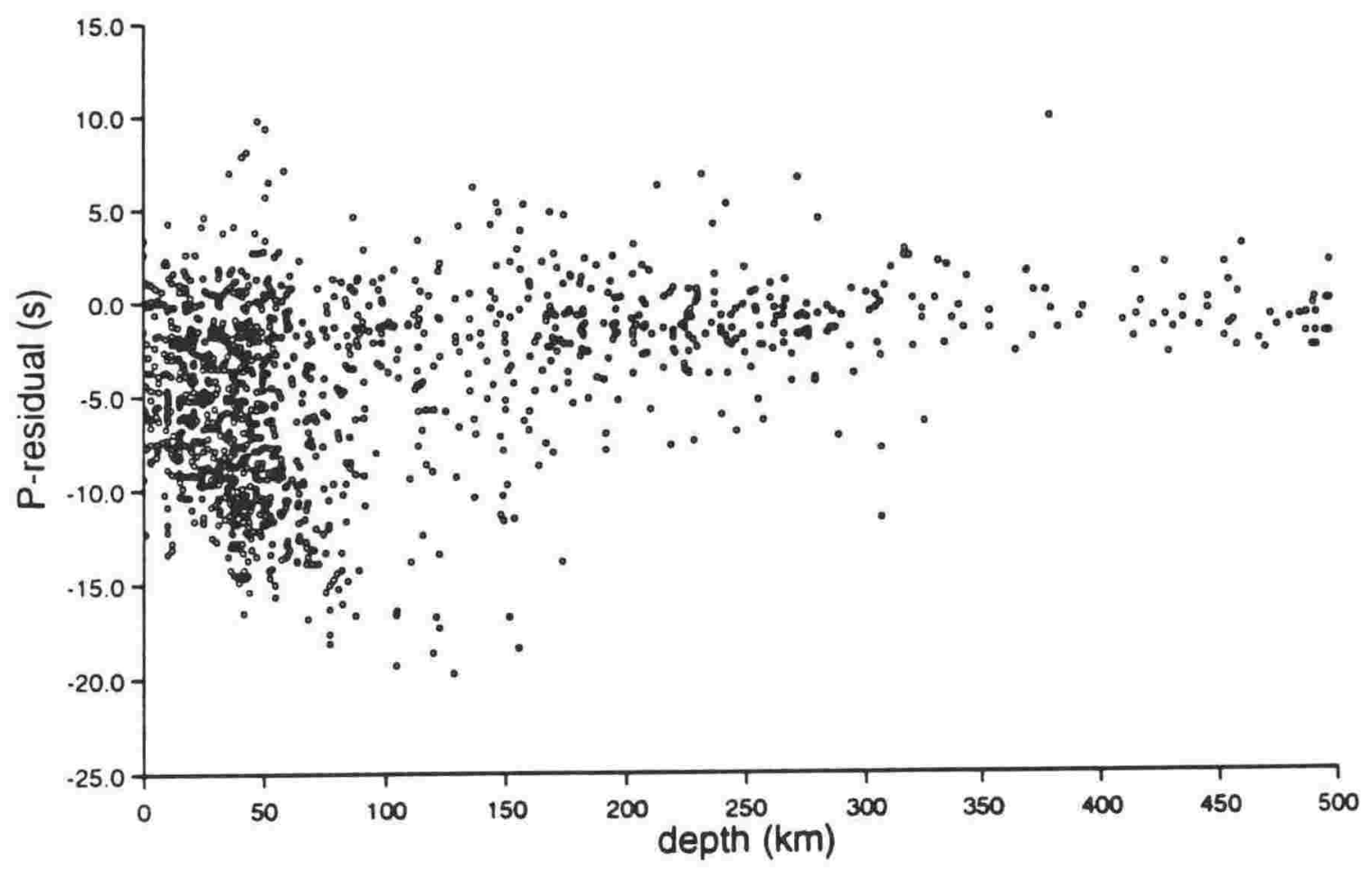

Fig. 6.2 P-residuals for all depths. 
later in this section, the coverage shown is restricted to paths whose distance does not exceed $17^{\circ}$. The available azimuth is thus limited slightly, extending from $30^{\circ}$ to $180^{\circ}$. important as it means that effects at one particular propagation azimuth are not attributable to one given station or source region. The marked reduction in the number of events at about $26^{\circ} \mathrm{S}$ is correlated with the lack of seismicity at that latitude within the Tonga-Kermadec trench, and similarly for the gap at $34-36^{\circ} \mathrm{S}$.

Figure 6.4 is a graph of travel time against distance, reduced at $8.00 \mathrm{~km} / \mathrm{s}$, for all available data after the selection process described above. Also shown in the figure are the JB travel time curves (Jeffreys \& Bullen, 1967) for source depths in the relevant range. It is seen that for paths longer than about $1900 \mathrm{~km}$, or $17^{\circ}$, the travel times coincide well with the JB times, corresponding at these distances to first-arriving diving waves bottoming deeper than $400 \mathrm{~km}$. For shorter paths, the first arrivals are generally earlier than the JB times, and are best explained

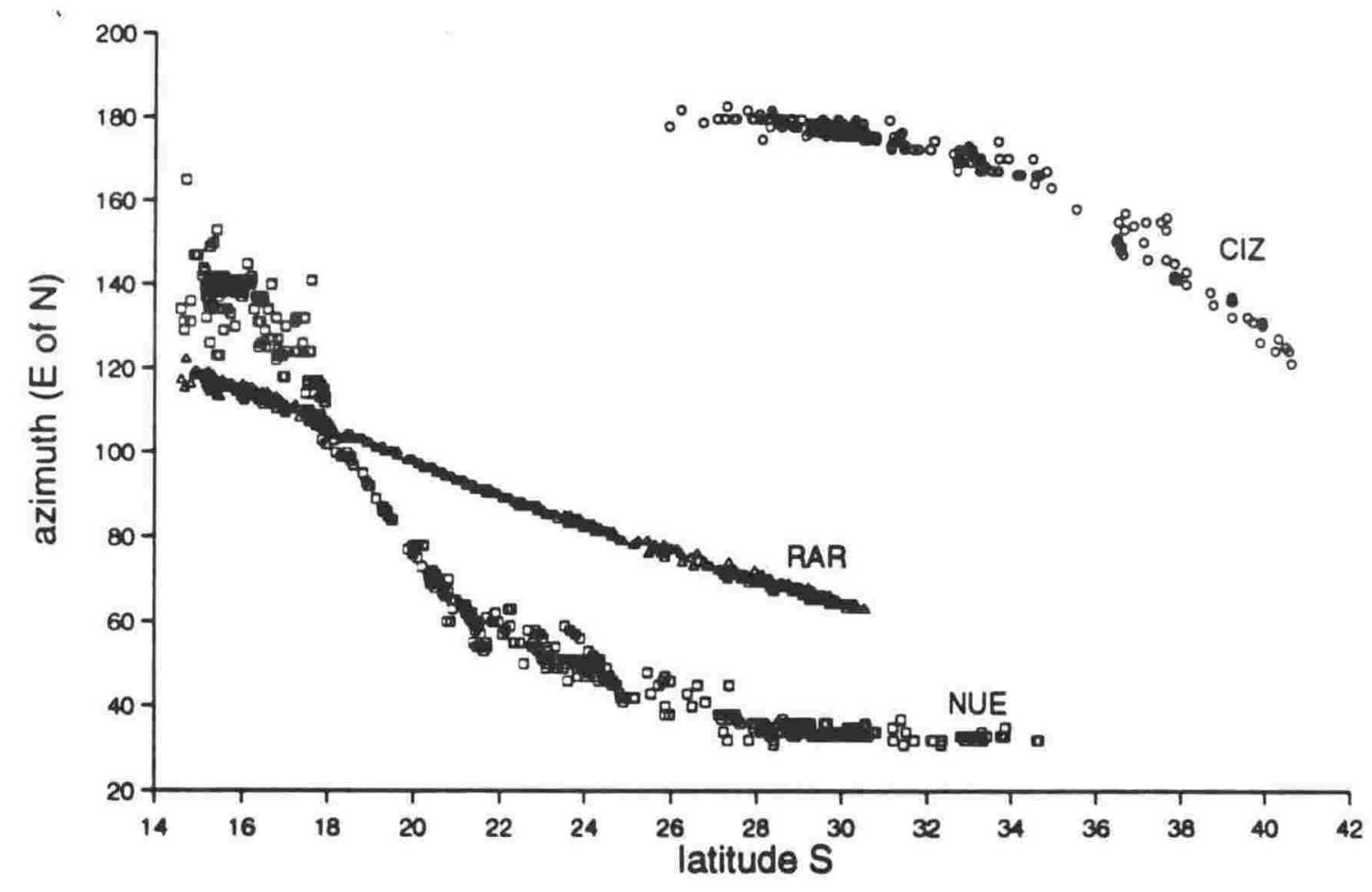

Fig. 6.3 Azimuth and latitude coverage by CIZ, NUE and RAR for path lengths shorter than $17^{\circ}$. 
as "guided" through a high-velocity lid. This effect was modelled by Prasad and Bock, using a $100 \mathrm{~km}$-thick lid of velocity $8.3 \mathrm{~km} / \mathrm{s}$. Barazangi et al (1972) have also shown that the suboceanic lithosphere east of the Tonga trench, together with the subducted slab west of the trench, acts as an efficient wave-guide, capable of propagating waves to distances greater than $14^{\circ}$. This lithosphere is underlain by a low-velocity asthenosphere, so that for the ray paths considered here, waves propagating through the "lid" would arrive before the diving waves through the asthenosphere. Regan and Anderson (1984), by inversion of surface wave data, arrive at generalized models of the oceanic lithosphere having an approximately $50 \mathrm{~km}$-thick, anisotropic lid, of mean P velocity $8.3 \mathrm{~km} / \mathrm{s}$, in the case of old Pacific crust. While not attempting to model the thickness and structure of such a lid in this region, the existence of anisotropy within it is here investigated. Thus, using the graph of Figure 6.4, only those paths were selected whose length was shorter than $17^{\circ}$. This further selection left a total of 926 suitable individual observations in the data set.

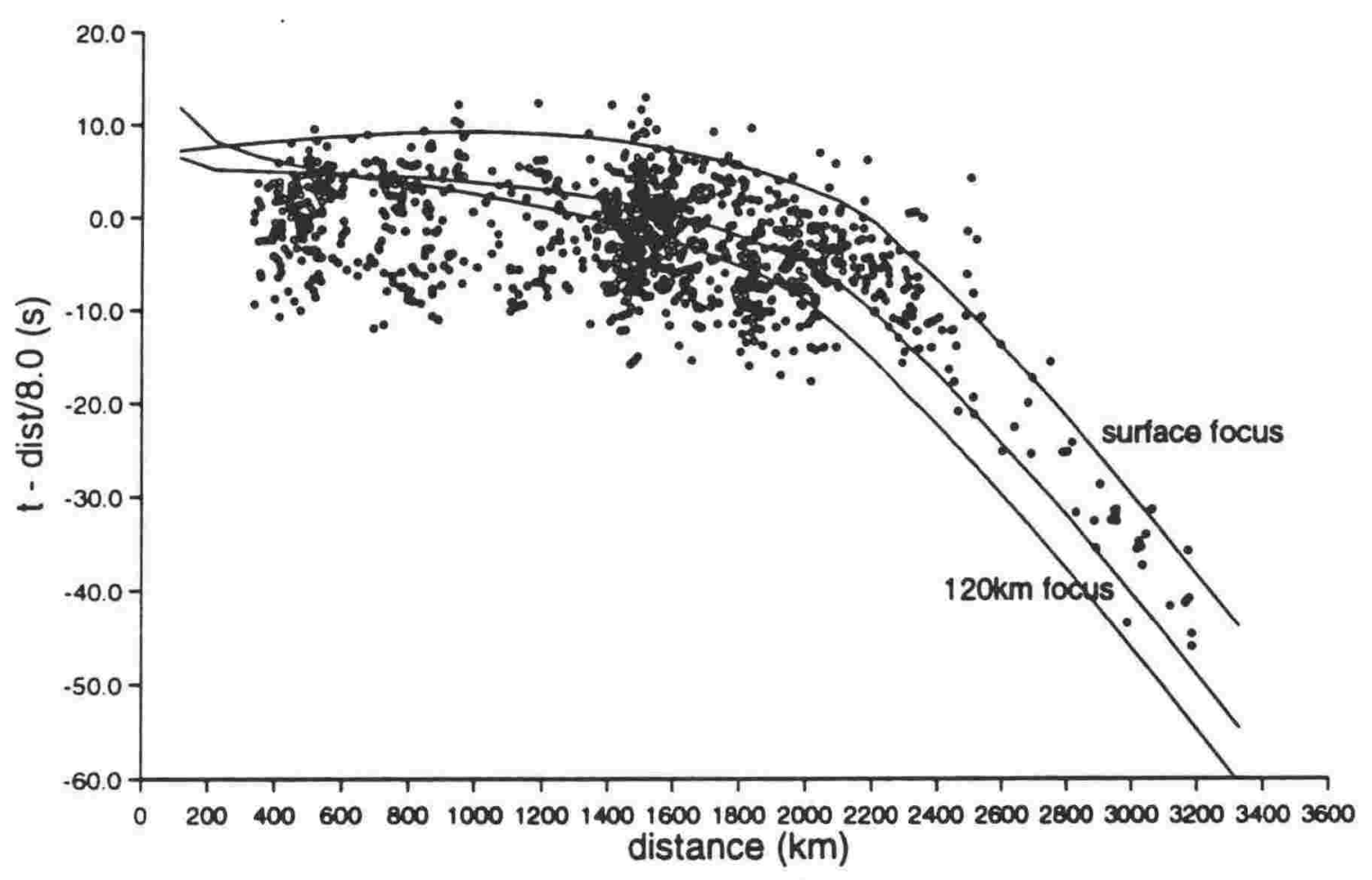

Fig. 6. 4 Reduced travel time plot for all path lengths, and for shallow $(<100 \mathrm{~km})$ events. 


\subsection{Azimuthal Dependence of P-residuals and P-velocities}

Figure 6.5 shows the variation of P-residuals (observed - JB travel times) with azimuth for path lengths shorter than $17^{\circ}$. A variation of the residuals with azimuth is indicated, but a large scatter still exists. Errors in hypocentre location, origin time and seismogram reading partly account for the scatter. Subduction zone earthquakes may be mislocated by several tens of kilometres (Ansell,1978). Therefore it is possible that some of the deepest events in the data set actually belong to the class of earthquakes deeper than $100 \mathrm{~km}$, which produce small, or zero, Presiduals, although, as discussed in the previous section, this should account for only a small percentage of the scatter.

An interesting observation, which does account for much of the scatter, is that from any one azimuth, there is a general increase of the magnitude of the residuals with earthquake depth within the $0-100 \mathrm{~km}$ depth range. This trend can be seen in Figure 6.2 for the whole data set, but

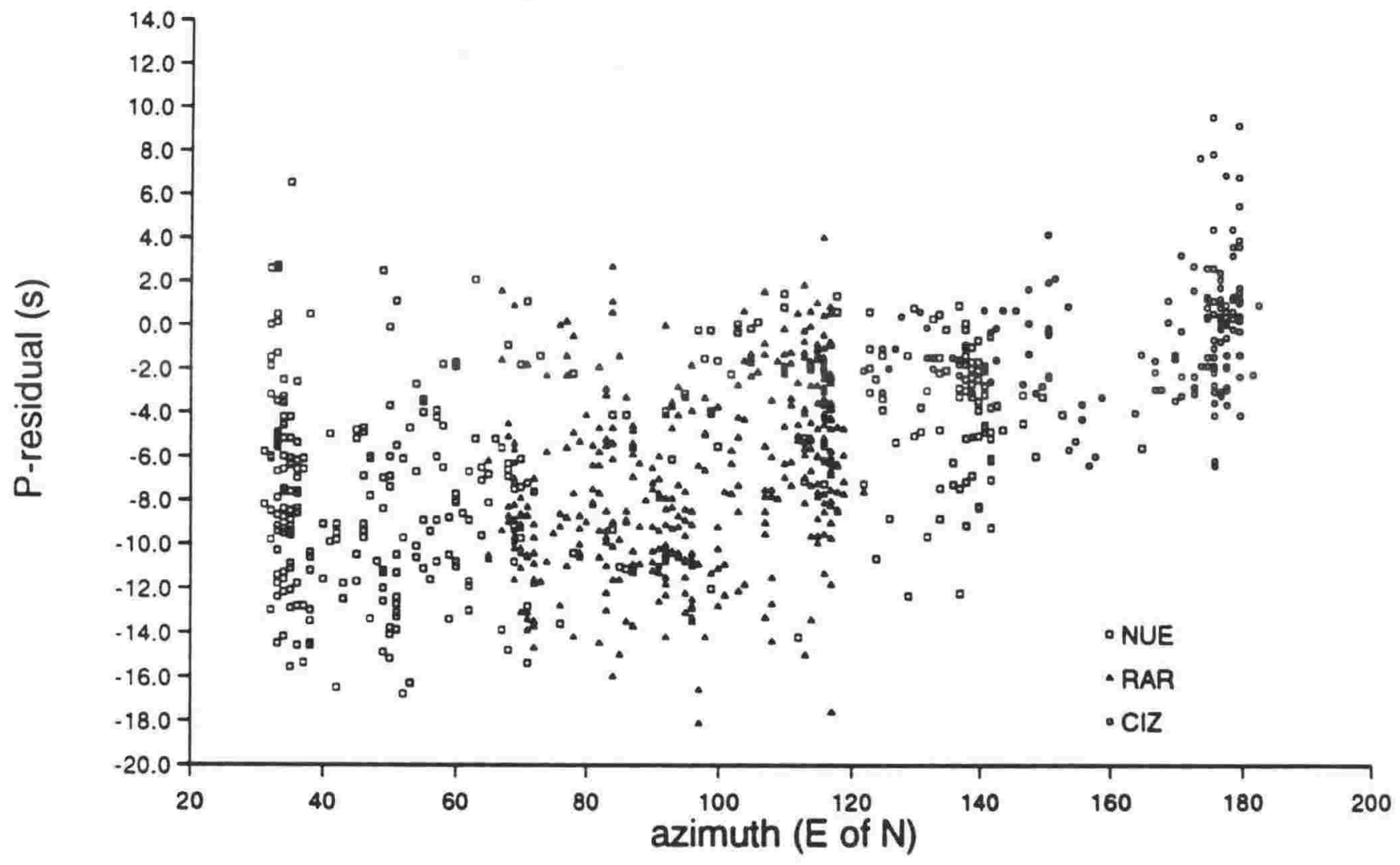

Fig. 6.5Variation of $P$-residual with propagation azimuth for events in the selected data set. Different stations are represented by different symbols. 
is also reflected in the variation of average path velocity with source depth, and will be referred to again later on. In spite of the scatter however, there is a clear overall trend in Figure 6.5, with the magnitude of residuals decreasing towards larger azimuths.

A better way of investigating anisotropy was to plot P-velocities directly. This avoided the dependence of residuals on distance travelled and on standard travel time tables. Given that the path lengths were more than $300 \mathrm{~km}$ long, and that, in general, the sources were presumably close to the surface of, or within the subducted slab (Mitronovas et al,1969), it is reasonable to assume that the first-arriving waves travelled for most of their path along the high velocity lid, and therefore that the average velocity (distance/travel time) would be close to the propagation velocity within the lid. However, it was decided to apply a static correction to the receiver end of the paths, although this must be considered as a very imprecise approximation. CIZ is known to lie above a thick crust (17 - $23 \mathrm{~km}$ ) (Adams, 1962), in an area where the water depth is less than $1000 \mathrm{~m}$. The crust east of Tonga trench, on the other hand, is reported by Raitt et al (1955) to have a normal oceanic thickness of about $12 \mathrm{~km}$, and modelled by Shearer and Orcutt (1985) as being $8 \mathrm{~km}$ thick. The islands of Niue and Rarotonga rise from a water depth of about $5000 \mathrm{~m}$. It was thus decided to apply an average correction for $20 \mathrm{~km}$ of crust, having a mean P-velocity of $6.5 \mathrm{~km} / \mathrm{s}$, below CIZ, and $15 \mathrm{~km}$ below NUE and RAR.

Figure 6.6 shows all the velocity values obtained, using different symbols for the different stations. The larger, solid symbols are the corresponding averages for the respective stations computed at $10^{\circ}$ intervals. NUE shows by far the widest scatter of points. This is understandable in view of its proximity to the trench, especially for large azimuths, which correspond to the shortest distances in the data set. At these distances, errors in origin time, spatial location, phase reading as well as the static correction applied have a large influence on the simple velocity estimate made here. An error of $2 \mathrm{~s}$ in origin time, for instance, would produce a $0.4 \mathrm{~km} / \mathrm{s}$ change in the velocity at a distance of $300 \mathrm{~km}$, but of less than $0.1 \mathrm{~km} / \mathrm{s}$ at a distance of $1500 \mathrm{~km}$, as in the case of RAR. Bock (1981) accurately relocated a number of earthquakes in the Tonga region, and for the shallow ones his origin times are an average of $0.6 \mathrm{~s}$ earlier than the I.S.C. times, with some differences as large as $2 \mathrm{~s}$. Some improvement to the NUE points was made by further selecting only those picks which were classified as iP, thus reducing the uncertainty in travel time.

Apart from the irregular scatter of NUE, however, there also exists a systematic variation of the average $P$ velocity with source depth, in correspondence with the variation of residuals previously referred to. As an example, Figure 6.7 shows the velocities measured at RAR from the narrow azimuth range $80-90^{\circ}$. The variation in measured velocity is hard to explain in terms of location and observational errors, and is observed for all 3 stations, for most azimuths, except for those corresponding to events at the northernmost end of the trench. The increase in velocity with source depth, is larger than that predicted by the JB travel times for the direct P-wave at the corresponding distances. It is conceivable that some kind of velocity structure within the lid may 


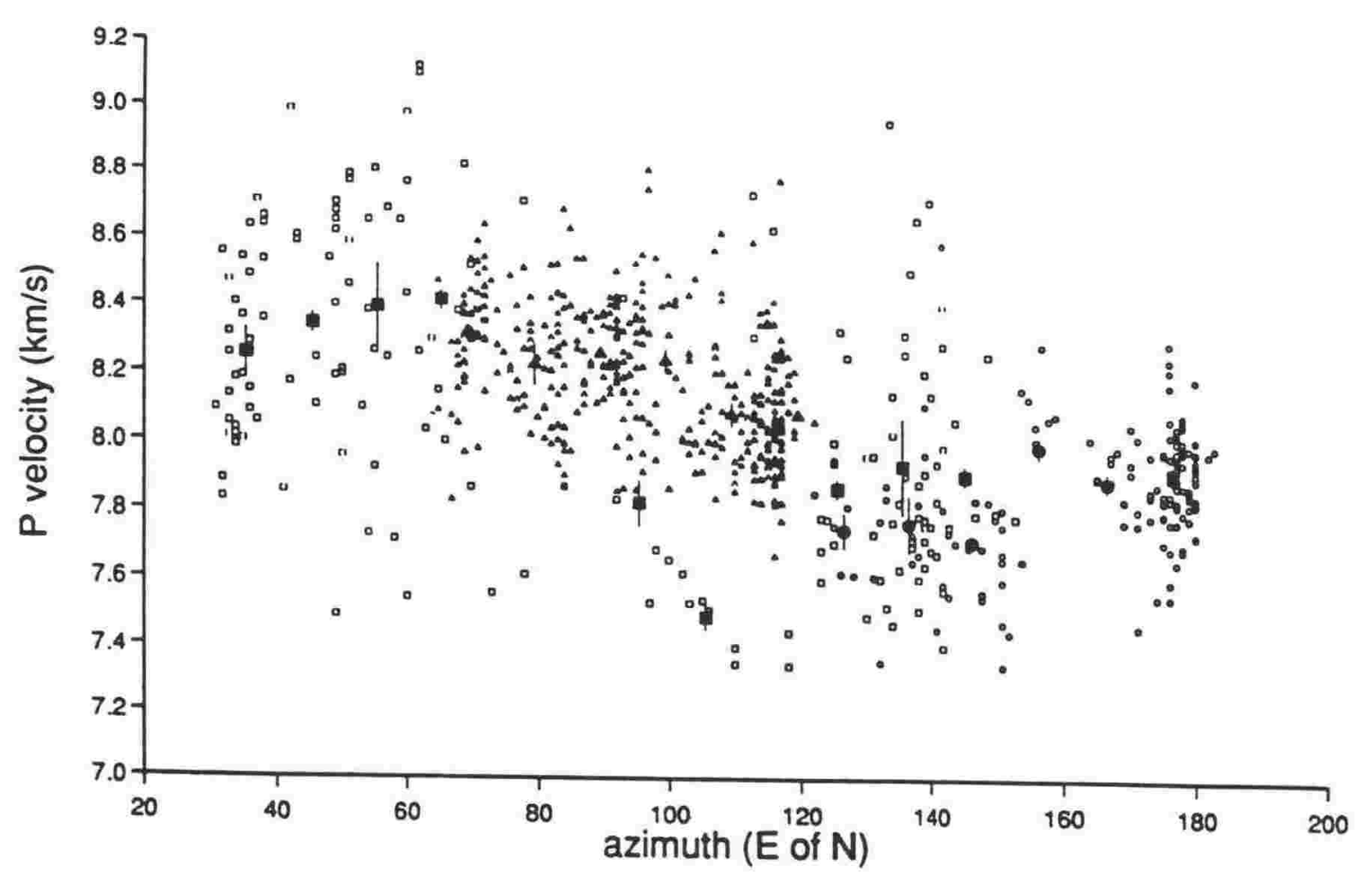

Fig. 6.6P-velocity, as measured at the three stations. Symbols as in Fig.6.5. Large, solid symbols are averages at individual stations at $10^{\circ}$ intervals.

be responsible for this effect, however no rigorous attempt at modelling or explaining it has been made in this study. For the purpose of investigating azimuthal variations in velocity, it is sufficient to recognize the dominant trend over the whole azimuth range, and to accept that the source depth effect is partly responsible for the observed scatter. The linearity of the variation implies that taking the average velocity at a given azimuth, as discussed below, is approximately equivalent to reducing the data to a source of average depth. For the whole data set, this is around $40 \mathrm{~km}$.

Figure 6.8 shows the average velocities for all data points, computed at $5^{o}$ intervals. Assuming that the variation is due to anisotropy in the upper mantle, the points are expected to lie on a sinusoid, with the maximum and minimum values $90^{\circ}$ apart. Following Backus (1965), weak anisotropy $(<10 \%)$ leads to a variation of velocity with azimuth of the form 


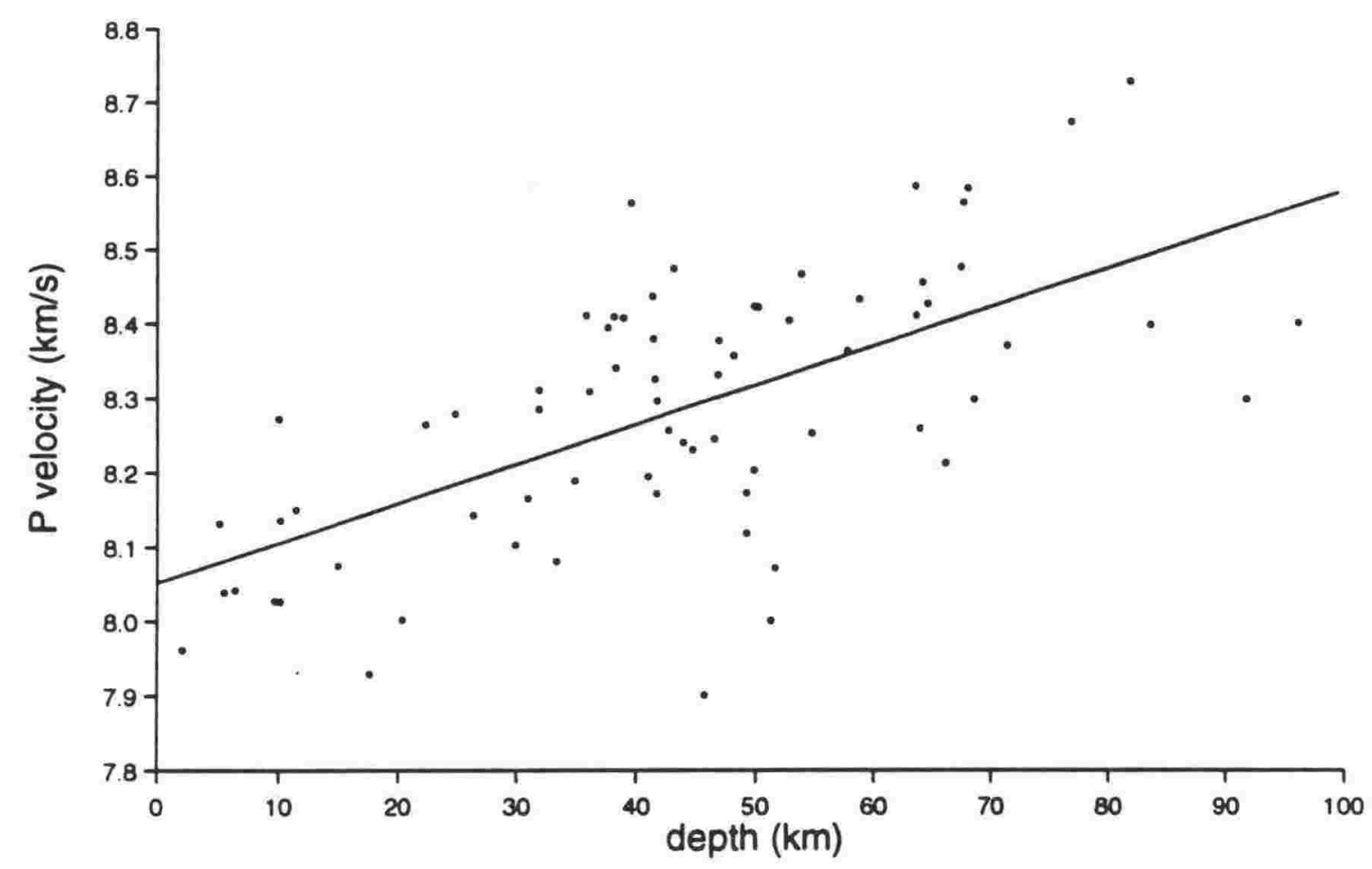

Fig. 6. 7 Velocities measured at RAR from events in the azimuth range $80-90^{\circ}$, showing dependence on source depth. The line of least-squares fit has the equation $v=8.05+0.0053 \mathrm{~d}$.

$$
v=v_{o}+A \sin 2 \phi+B \cos 2 \phi+C \sin 4 \phi+D \cos 4 \phi
$$

where $v_{o}$ is the average velocity, $v$ is the velocity along a propagation azimuth $\phi$, and $A, B, C$ and $D$ are constants related to the elastic constants of the medium. For oceanic anisotropy, the coefficients $C$ and $D$ are generally much smaller than $A$ and $B$, and were dropped in this case. Thus, an equation of the form

$$
v=v_{o}+A \cos 2 \phi+B \cos 2 \phi
$$

was fitted by a least-squares method, which yielded the solid curve in Figure 6.9.

For this curve, the constants of equation (2) are: $v_{o}=8.08 \mathrm{~km} / \mathrm{s}, A=0.242 \mathrm{~km} / \mathrm{s}$ and $B=-0.150$ $\mathrm{km} / \mathrm{s}$ and the velocity $v$ may be written as

$$
v=8.08+0.285 \cos (2 \phi-124) \pm 0.06
$$

This is equivalent to a velocity maximum of $8.37 \mathrm{~km} / \mathrm{s}$ at an azimuth of $\mathrm{N} 62^{\circ} \mathrm{E}$, and a minimum 


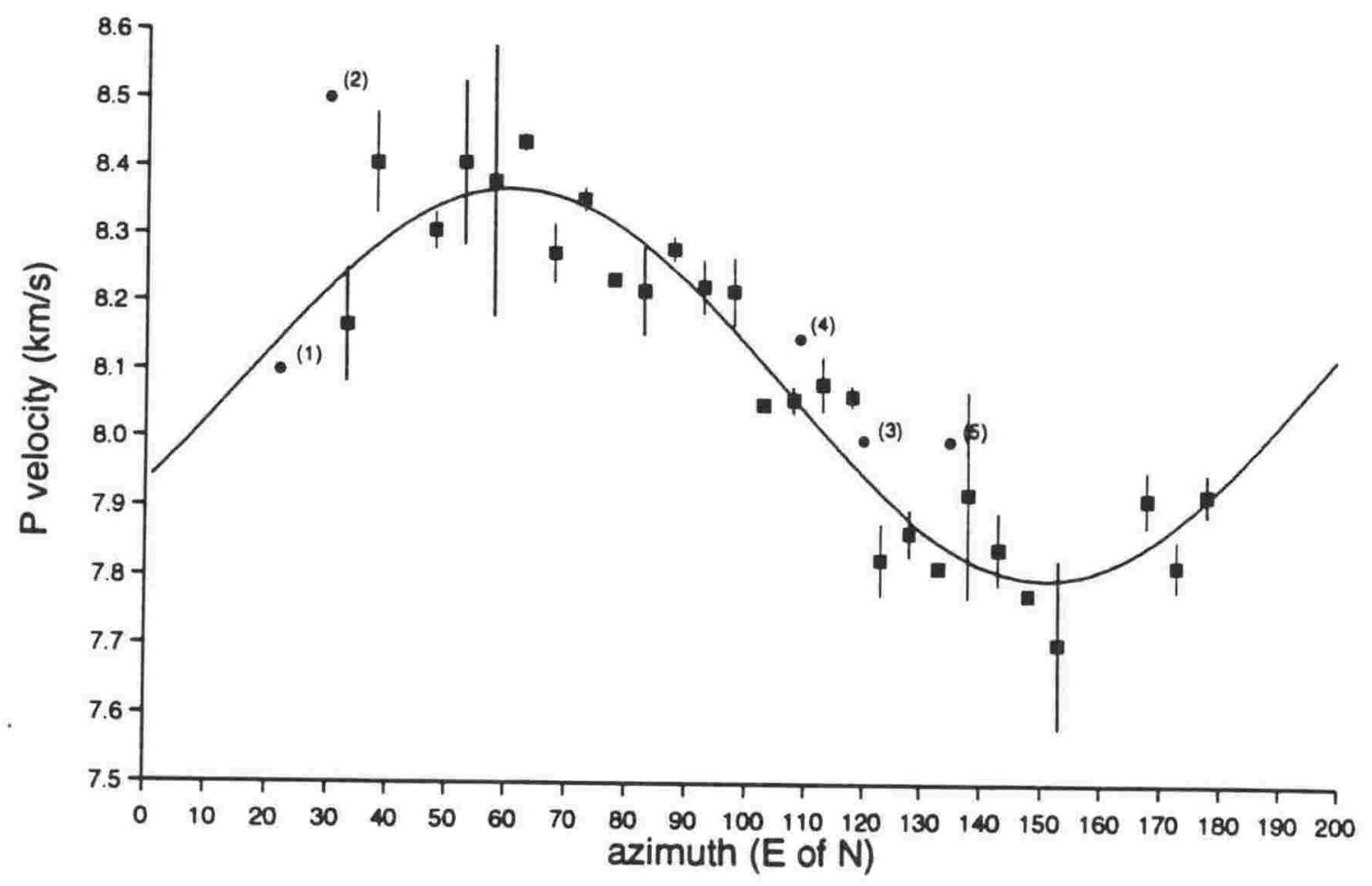

Fig. 6.8Mean $P$-velocity calculated at $5^{\circ}$ intervals (square symbols) and associated standard deviation of the data scatter. The solid curve is the least-squares best-fit sinusoid. Numbered solid circles represent previous measurements of $P$-velocity in the same area - (1) Raitt et al (1955), east of Tonga trench; (2) Shearer and Orcutt (1985) near Niue (fast axis); (3) Shearer and Orcutt (1985) near Niue (slow axis);

(4) This study, average interval velocity between NUE and RAR; (5) Haines (1979), mean $\mathrm{P}_{n}$ velocity from North Island, New Zealand to CIZ

of $7.80 \mathrm{~km} / \mathrm{s}$ at $\mathrm{N} 152^{\circ} \mathrm{E}$, an anisotropy of approximately $7 \%$.

Figure 6.9 represents the same data as Figure 6.8 , but divided into the depth ranges $0-50 \mathrm{~km}$ and $50-100 \mathrm{~km}$. The source depth effect is clearly visible.

A further calculation was carried out using events on the Tonga trench which were approximately collinear with the NUE-RAR line and computing the interval velocity from the arrival time difference between the two stations. Such a value is much less influenced by mislocations and origin time errors, and thus should be more truly representative of the Pvelocity along that direction. The average value yielded by this calculation was $8.20 \pm 0.11 \mathrm{~km} / \mathrm{s}$ at an average azimuth of $108^{\circ}$. However, within this data set, the average interval velocity for 
source depths less than $50 \mathrm{~km}$ was $8.15 \pm 0.12 \mathrm{~km} / \mathrm{s}$, while for depths between 50 and $100 \mathrm{~km}$, the average velocity was $8.25 \pm 0.12 \mathrm{~km} / \mathrm{s}$. The value of $8.20 \mathrm{~km} / \mathrm{s}$ is larger than the value of $8.07 \mathrm{~km} / \mathrm{s}$ predicted by the least-squares curve but is within the scatter of the single-station velocity data at this azimuth.

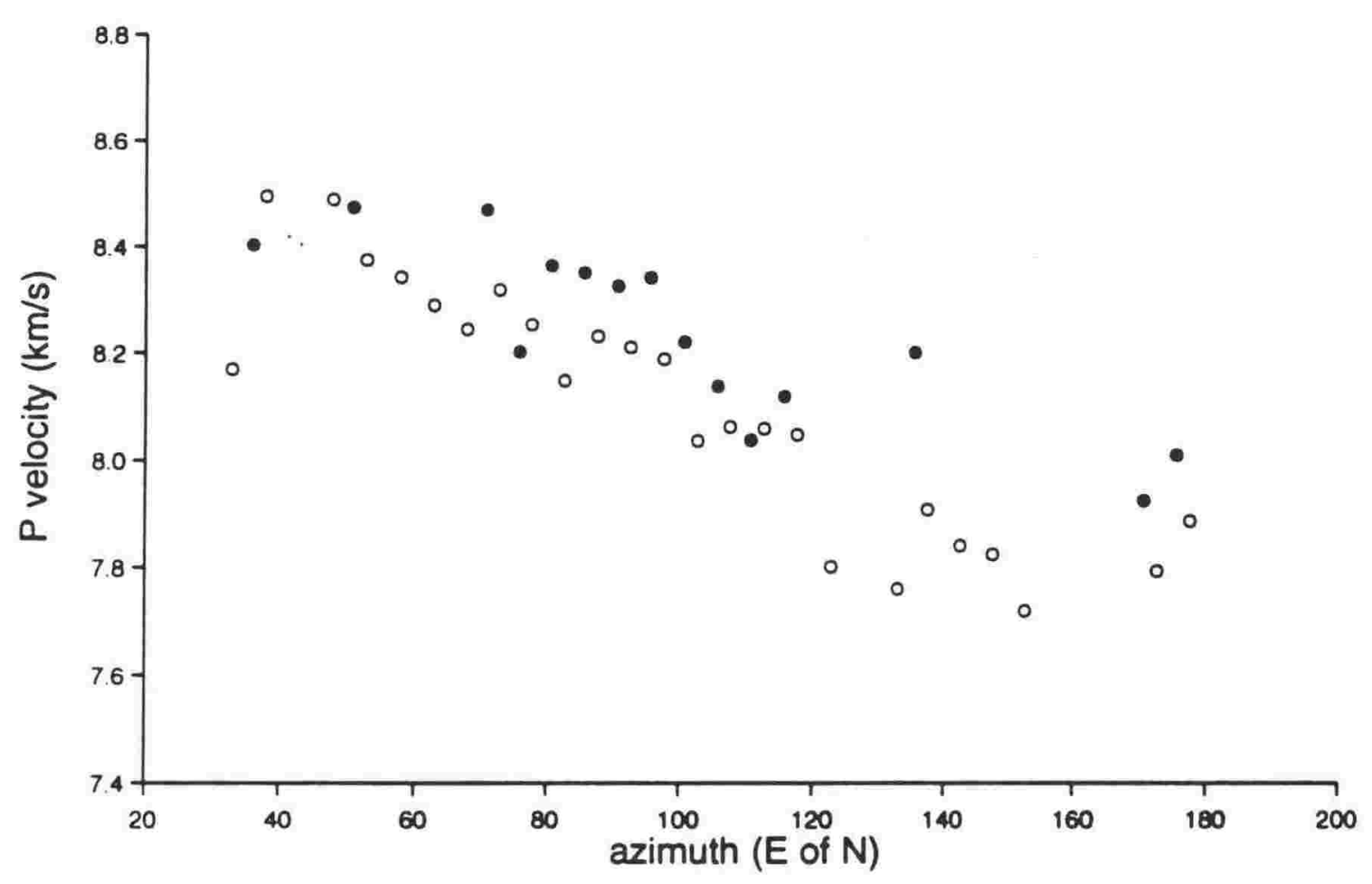

Fig. 6.9As Figure 6.8, but events are grouped into $0-50 \mathrm{~km}$ and $50-100 \mathrm{~km}$ depth ranges. Open circles are the velocities from the shallow sources; filled circles are the velocities from the deeper sources. 


\subsection{Implications and Discussion}

The variation of average P-velocity with azimuth in Figure 6.8 appears to be reasonably consistent with a sinusoidal variation, and hence with the existence of anisotropy in the upper mantle. Admittedly, the area and azimuths covered by paths to CIZ do not overlap to any great extent with those covered by paths to NUE and RAR. In addition, the upper mantle between CIZ and the North Island, New Zealand is possibly different in character from that further north, being overlain by "thickened crust", intermediate between the Pacific oceanic crust to the north, and the more continental-like crust of the Chatham Rise to the south. Therefore one cannot totally exclude the possibility that the observations are partly due to lateral variation within the Pacific lithosphere over the area covered. However, this applies only to azimuths larger than $140^{\circ}$. The azimuth range covered by NUE and RAR samples a common block of lithosphere and still reveals a distinct variation of velocity with propagation azimuth.

It is not possible from this data to further resolve the depth within the upper mantle at which the anisotropy exists. The scatter of velocities at most azimuths has been partly identified with a systematic variation of the average path velocity with source depth. This is a very interesting observation which, on further investigation, should yield more information on the detailed structure, and therefore warrants further study.

The velocity variation obtained in this study is consistent with previous observations in this area. Some of these are plotted on Figure 6.8 for comparison. The observations which have been included here are restricted to measurements made on the pre-subducted, horizontal Pacific plate. Measurements of P-velocity made directly along strike of the Tonga - Kermadec trench typically yield even higher velocities, of the order of $8.4 \mathrm{~km} / \mathrm{s}$ (e.g. Ansell and Gubbins, 1985; Aggarwal et $a l, 1972)$. The direction of maximum velocity obtained here $\left(\mathrm{N} 60^{\circ} \mathrm{E}\right)$ is different from that of Shearer and Orcutt (1985) who found it to be closer to N30 ${ }^{\circ} \mathrm{E}$. The experiment of Shearer and Orcutt, however. was confined to a limited area, as opposed to the much larger extent represented by the present results. The value of $7 \%$ for the degree of anisotropy is very close to that of Shearer and Orcutt, as well as to the value of $8 \%$ found near Hawaii by Morris et al (1969). Taken together, the results point to a fossil spreading direction in a general south-westerly direction, as opposed to the present north-westerly direction of transport of the south west Pacific plate. Such a difference in directions has also been observed by Montagner and Tanimoto (1991). The authors find, for example, that the azimuthal anisotropy of shear waves in the upper $100 \mathrm{~km}$ of the Pacific ocean is different from that deeper down. They associate the shallow anisotropy with fossil sea-floor spreading, and the deeper one with present day plate motion.

The possible relationship between the high velocities found within the subducted plate below New Zealand, and the anisotropic upper mantle in the stable Pacific lithosphere will be discussed in the next and final chapter. 


\section{Chapter 7}

\section{Summary and Discussion of Results}

\subsection{Main Results}

The main results that have emerged from this study are:

(i) Material characterised by high seismic P-wave velocities, $8.75 \pm 0.10 \mathrm{~km} / \mathrm{s}$, exists within the subducted Pacific lithosphere below the North Island, New Zealand. These velocities were calculated by inverting array arrival times from a number of $P_{n}$ pulses from a shallow aftershock sequence (Weber data set) to solve for wavefront speed and azimuth. The apparent velocity of the wavefront was $8.7 \pm 0.1 \mathrm{~km} / \mathrm{s}$. The value of $8.75 \mathrm{~km} / \mathrm{s}$ for the refractor velocity was arrived at after taking into account the geometry of the slab through which the waves travel.

As a result of the above inversion, the off-azimuth incidence of $\mathrm{P}$-waves travelling almost along strike of the subducted slab and undergoing lateral refraction off the slab before arriving at Wellington has been clearly demonstrated. Both the wavefront speed and azimuth have been shown to be sensitive to the exact nature of the curvature of the slab. This effect was modelled using 3-dimensional ray tracing. The plate geometry proposed by Ansell and Bannister (1991), which incorporates a slight updip component along strike, has been found to account satisfactorily for the observations. Using this geometry, a lower inherent refractor velocity is required to explain the observed $P_{n}$ wavefront characteristics, compared with a plane, or regular cylindrical slab, which would necessitate $P$-wave velocities of at least $8.9 \mathrm{~km} / \mathrm{s}$ within the upper mantle.

(ii) The major characteristics of the waveforms have been satisfactorily modelled using synthetic seismograms generated by a reflectivity technique (Kennett, 1983). These waveforms have been produced by waves travelling almost along strike of the gently dipping subducted slab, and therefore the approximation of horizontal layering has been made. They have been shown to be consistent with a layered upper mantle containing approximately $6 \pm 2 \mathrm{~km}$ of high velocity material at depths of $18-20 \mathrm{~km}$ below the surface of the subducted slab, underlying an $8.2 \pm 0.2$ $\mathrm{km} / \mathrm{s}$ uppermost mantle. The simple, distinct, $P_{n}$ pulse propagates through this layer, and the high frequency signal immediately following $P_{n}$ propagates through the overlying layers as a sequence of reflections and refractions. Large amplitude, lower frequency second arrivals, dominating the P-wavetrain of most seismograms, have been modelled as reverberatory phases in a crustal waveguide.

(iii) The waveform modelling has shown that a layer of low-velocity material separating the 
crustal layers and the subducted lithosphere produces a highly characteristic signal in the Pwavetrain of the synthetic seismogram. The signal has the form of high amplitude reverberations in the later part of the P-coda. Such a signal is not observed in the seismograms of the Weber data set, but is seen in seismograms for which the wave travel path is further along the East Coast. This suggests either that the presence of the low-velocity layer (presumably representing subducted ocean sediment) is indicated along the East Coast but not along the paths considered, or that the curved geometry of the plate surface interferes in some way with the generation of this particular phase.

(iv) A separate, self-contained study, using I.S.C. travel time data from earthquakes on the Tonga-Kermadec trench recorded at 3 South Pacific stations has revealed the existence of upper mantle P-wave anisotropy of around 7\% in the area of the south west Pacific enclosed by the trench, Rarotonga, the Chatham Islands and the North Island, New Zealand. The $P_{n}$ velocity in this region varies from $7.80 \mathrm{~km} / \mathrm{s}$ in a direction $\mathrm{N} 152^{\circ} \mathrm{E}$ to $8.37 \mathrm{~km} / \mathrm{s}$ along $\mathrm{N} 62^{\circ} \mathrm{E}$.

\subsection{Petrological implications of the high velocities}

\subsubsection{The oceanic lithosphere}

Whereas the continental lithosphere appears to possess gross lateral heterogeneity, the oceanic lithosphere is more uniform in character, and, as a result, geophysicists and geologists now agree upon a fairly consistent model of its structure, composition and behaviour. Even so, with the advent of high resolution seismic profiling, the finer details still yield new insights and highlight regional peculiarities.

The uprise of material at mid-ocean ridges is accompanied by partial melting and chemical differentiation to produce a zoned lithosphere consisting of a basaltic crust overlying a peridotitic lid (Ringwood, 1982) which has olivine as the dominant component. Figure 7.1, taken from Ringwood (1982), illustrates the chemical structure proposed by that author. Estimates of composition of the various layers vary from author to author as well as regionally. Anderson (1979), for example, proposes a rather different model for the upper mantle down to the $470 \mathrm{~km}$ discontinuity, having a higher proportion of eclogite.

Studies of ophiolites (mantle material that has been thrust to the surface) suggest that the top $20 \mathrm{~km}$ of oceanic mantle have an average of $75 \%$ olivine, $20 \%$ orthopyroxene (OPX) and $5 \%$ spinel (Nicolas and Christensen, 1987), but that this layer may be locally dunitic, having as much as 93\% olivine (Christensen and Ramananantoandro, 1971; Nicolas and Christensen, 1987). When dredging along the Tonga trench, Bloomer and Fisher (1986) recovered a sample of 


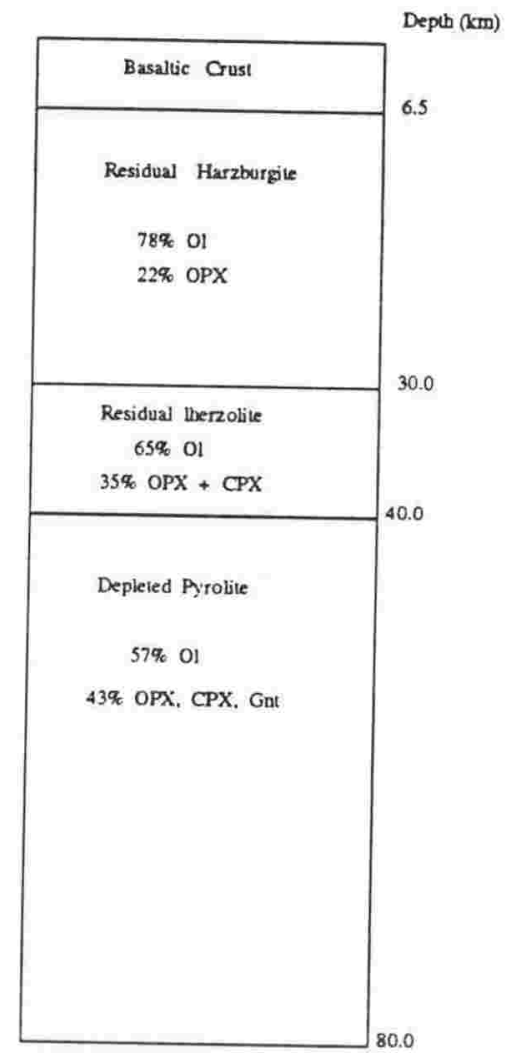

Fig. 7.1 Zonation of the oceanic lithosphere (Ringwood, 1982)

The basaltic crust itself acquires a layered structure during cooling of the newly extruded magma. Deep sea drilling, as well as measurements of seismic velocities from reflection and refraction surveying, have generally revealed the oceanic crust to be composed of 3 main layers a sediment layer, a basalt layer and a transitional layer composed mainly of gabbro (Coleman, 1971; Suyehiro, 1988). Figure 7.2, taken from Hsü (1987), shows one interpretation of the ocean crust layering.

A review of seismic velocity structure of ocean lithosphere in various regions of the world is given in Suyehiro (1988). In this review it is shown that the average P-velocity below the Moho in oceanic regions is about $8.2 \pm 0.2 \mathrm{~km} / \mathrm{s}$. P-velocities well in excess of this value have been reported, however, in several places around the world, as will be described later in this chapter. The possible explanations for the high velocities found in this study are now discussed. 


\subsubsection{Eclogite and olivine as candidates for high $P$-velocities}

The subduction of oceanic lithosphere at a convergent plate boundary, such as the TongaKermadec trench system, brings about large changes in the conditions of temperature and pressure and in the ambient stress field. One effect of these changes is that they give rise to phase transformations of the various minerals making up the oceanic crust and mantle. A good review of such processes is given in Ringwood $(1976,1982)$. The first major transformation that occurs on subduction is that basalt in the oceanic crust transforms to eclogite at depths of about $80 \mathrm{~km}$ (Ringwood, 1982). Eclogite contains a high percentage of the mineral garnet (up to $51 \%$ ) (Anderson, 1979) which accounts for its high seismic velocities and density. The proportion of garnet continues to increase with depth, corresponding to the increase in pressure. The harzburgite (olivine + OPX + trace garnet) making up the upper mantle, on the other hand, does not begin to transform to its high pressure equivalents before depths of about $350 \mathrm{~km}$.

One candidate that might account for high seismic velocities within a subducted plate at

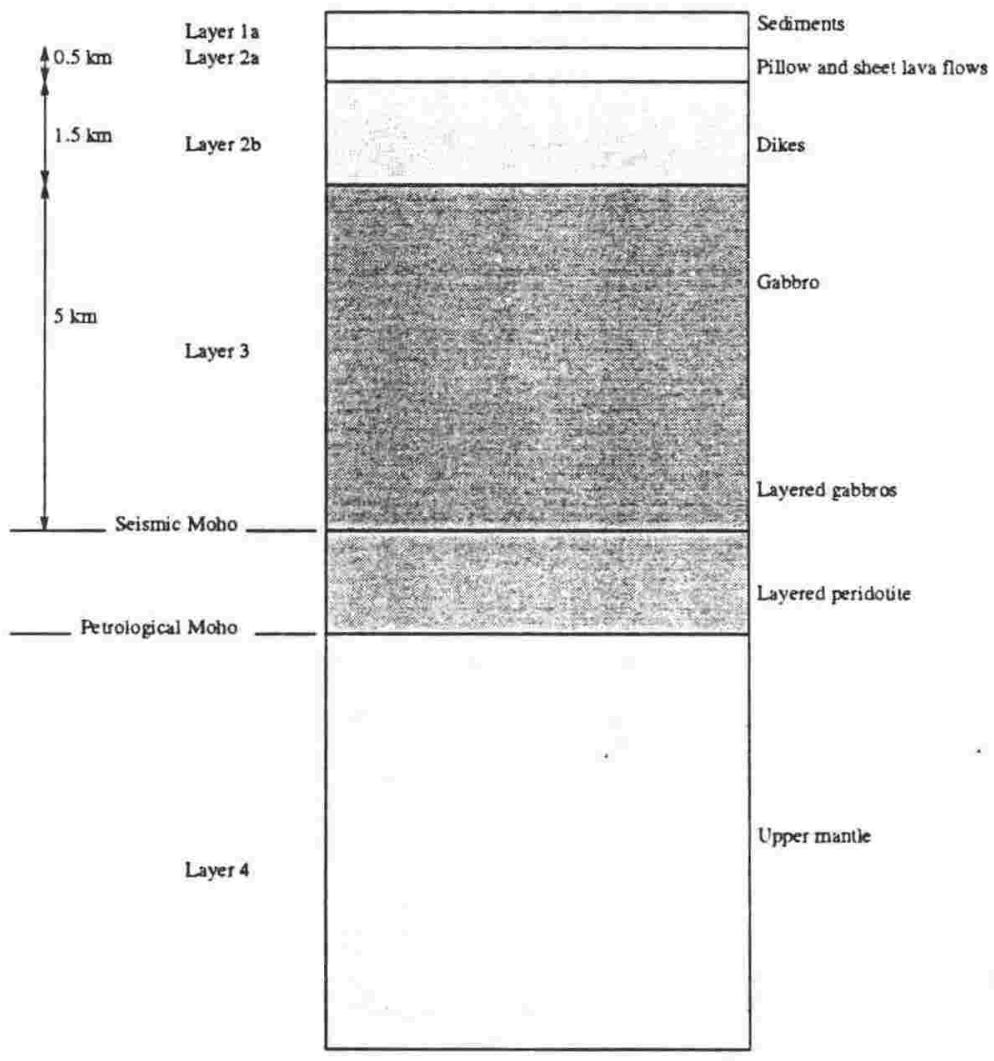

Fig. 7.2 Layering of the ocean crust (Hsï, 1987) 
relatively shallow depths would then be eclogite. Gubbins and Snieder (1991), from observations of fast, high frequency precursory P-waves travelling down the subducted Pacific plate below the Tonga-Kermadec trench, infer the presence of eclogite at quite shallow depths. Although this depth is not explicitly stated in the paper, they infer from their waveform modelling that a thin, high velocity "lid" (approximately $8.4 \mathrm{~km} / \mathrm{s}$ ), of thickness $6-12 \mathrm{~km}$, should exist within the subducted lithosphere, probably bounded by velocity gradients, and that this lid must be present at depths shallower than $80 \mathrm{~km}$. They attribute the high velocity to the basalt - eclogite transition, favouring this hypothesis mainly because of the similarity in thickness of the lid to the thickness of the basaltic oceanic crust. Hori (1990), analysing secondary P and S phases, concludes that the basalt - eclogite transition within the subducting Philippine Sea plate beneath Japan takes place at a minimum depth of $60 \mathrm{~km}$.

However, in the case of the slab below the eastern North Island, New Zealand, where the present study was made, it is highly unlikely that the basalt - eclogite transition has begun to take place as the slab descends in this region at a low angle and hence remains shallow. Even if eclogite were to be present, it would not exhibit velocities greater than about $8.5 \mathrm{~km} / \mathrm{s}$. The Pvelocity in eclogite is strongly related to the proportion of garnet present, since garnet has a P. velocity close to $9.0 \mathrm{~km} / \mathrm{s}$ (Babuska, 1981). Anderson (1979) lists some measured velocities of mantle minerals of varying composition. From these data, a P-velocity in eclogite of $8.75 \mathrm{~km} / \mathrm{s}$ would require that the eclogite be composed almost entirely of garnet. In fact, average velocities of this order are reached within the mantle at depths of around $350 \mathrm{~km}$, where garnet is a major component.

Another reason for discounting eclogite as the source of the high velocities is that the modelling, both of arrival times and of waveforms, requires the high velocity material to be deeper than the subducted Pacific crust (Section 5.6), and therefore we are dealing with a mantle, rather than an oceanic crust effect.

The other, more likely candidate for producing seismic velocities of the order of $8.75 \mathrm{~km} / \mathrm{s}$ is olivine. The mean compressional wave velocity quoted for olivine is $8.48 \mathrm{~km} / \mathrm{s}$ at $10^{-3} \mathrm{kbar}$ (Christensen and Ramananantoandro, 1971; Anderson, 1979). However the most important characteristic of this mineral is its high degree of seismic velocity anisotropy, especially for compressional waves.

The classical measurements in the laboratory of P-wave velocities in single olivine crystals were carried out by Verma (1960). These measurements show a variation in P-wave velocity from $7.72 \mathrm{~km} / \mathrm{s}$ along the slow (crystallographic [010]) axis, to $9.89 \mathrm{~km} / \mathrm{s}$ along the fast (crystallographic [100]) axis, an anisotropy of $24 \%$ (Figure 7.3 ). The P-wave velocity along the intermediate axis is $8.43 \mathrm{~km} / \mathrm{s}$. Similarly, OPX demonstrates compressional wave velocity anisotropy of around $16 \%$, with its velocity maximum reaching $8.25 \mathrm{~km} / \mathrm{s}$ (Nicolas and Christensen, 1987).

Nevertheless, in a rock containing olivine, one would not expect to measure anisotropy 


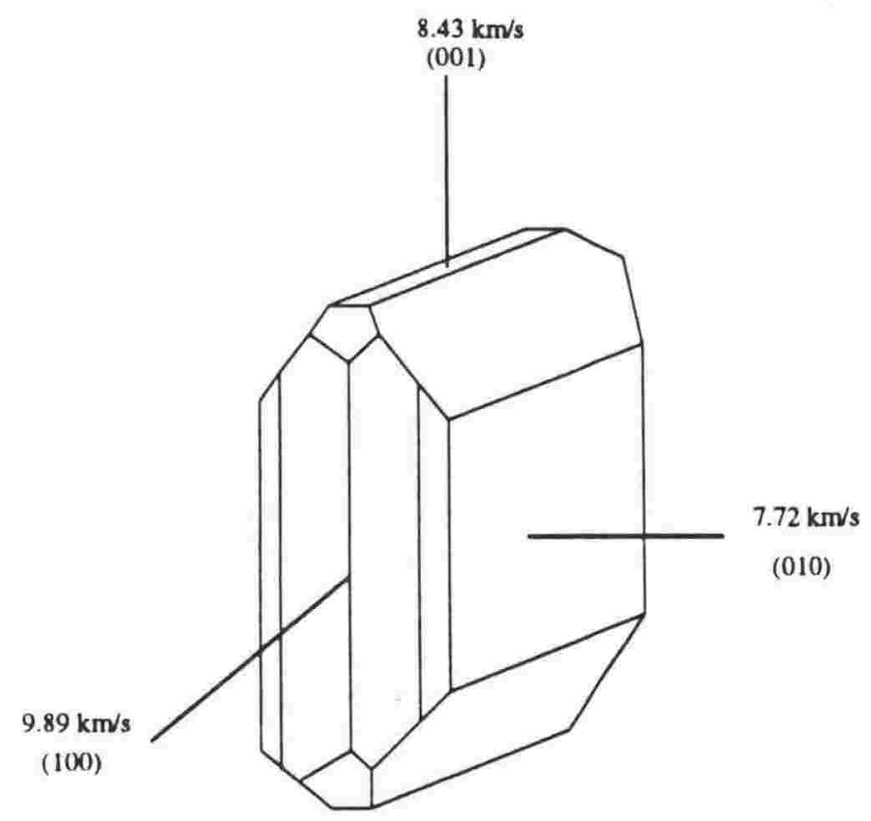

Fig. 7.3 Compressional wave velocities along crystallographic axes of olivine

unless some mechanism existed that allowed a considerable degree of alignment of the fast axes of individual crystals. One such mechanism was proposed by Hess (1964) - the plastic flow of spreading material in the vicinity of ocean ridges aligns the olivine (010) slip planes in the upper mantle minerals perpendicular to the ridge axis and parallel to transform faults. Since the maximum P-wave velocity in single crystals coincides with the dominant slip axis, then the "fast" direction in olivine aggregate rocks is directly equated with the flow direction. Other studies of such mechanisms include those of Forsyth (1977). Fuchs (1977) and Nicolas and Christensen (1987).

The degree of anisotropy in upper mantle rocks, as well as the values of $\left(\mathrm{V}_{\mathrm{p}}\right)_{\max }$ and $\left(\mathrm{V}_{\mathrm{p}}\right)_{\min }$ will be determined by both the proportions of olivine and OPX (Crampin and Bamford, 1977), as well as by the ductility, hence the degree of alignment of the rock. The strongest anisotropy (and consequently the largest $\mathrm{P}$-velocities) is expected in dunites and harzburgites, which contain $95 \%$ and $75 \%$ olivine respectively (Nicolas and Christensen, 1987). Analysis of ophiolites and core samples has revealed that dunites and harzburgites make up the major part of the upper mantle 
below the Moho discontinuity (Nicolas et $a l, 1980$ ) and that in this layer their strain is very high. Several studies have been carried out on mantle rocks in the form of ophiolites to confirm the postulate that single crystal anisotropy should lead to whole rock anisotropy via the spreading process. Some examples of these studies are those of Birch (1960), Christensen and Ramananantoandro (1971), Baker and Carter (1972), Christensen and Salisbury (1979), Boudier and Nicolas (1985) and Babuska (1981), in all of which studies, P-velocity anisotropy was reported and shown to be consistent with the theory of olivine alignment being parallel to spreading directions. Christensen and Ramananantoandro (1971) measured compressional wave velocities as high as $9.15 \mathrm{~km} / \mathrm{s}$ in naturally occurring dunite samples at $10.0 \mathrm{kbar}$ along the fast axis.

"In situ" measurements of P-velocities as high as $8.9 \mathrm{~km} / \mathrm{s}$ in the upper mantle, both in the context of upper mantle anisotropy as well as single measurements, have been recorded in the literature. Example are those of Den et al (1969), Hales et al (1970), Shimamura and Asada (1976), Asada et al (1983) and Sverev and Yaroshevskay (1987) for oceanic regions. Many researchers have come to the conclusion that the observed high $P_{n}$ velocities are hard to explain in terms of average P-velocities of normal mantle-forming minerals, even with eclogite. They can only be justified if the P-velocity anisotropy of olivine is taken into account (Carter et al, 1972; Fuchs, 1977; Crampin and Bamford, 1977).

\subsubsection{Likely mechanisms for the generation of high P-velocities in the subducted slab}

The most likely explanation for the $8.75 \mathrm{~km} / \mathrm{s}$ P-wave velocity found at relatively shallow depth below the North Island, New Zealand, is then that we are dealing with an anisotropic layer, within an olivine rich peridotite, with the fast axis being aligned along strike of the subducted slab. There remain, however, some important questions to be answered.

The Pacific plate, before it subducts, has been shown to have an anisotropic upper mantle (Chapter 6), with the fast axis being aligned approximately $\mathrm{N} 60^{\circ} \mathrm{E}$ and a maximum P-velocity of $8.37 \mathrm{~km} / \mathrm{s}$. The strike of the subducting Pacific plate below the North Island, on the other hand, is $\mathrm{N} 45^{\circ} \mathrm{E}$. If we are to assume that the value of $8.75 \mathrm{~km} / \mathrm{s}$, along strike of the slab, is the maximum P-velocity in an anisotropic medium, and that this medium is continuous with that of the Pacific ocean, there is a discrepancy both in the direction as well as in maximum velocity between the stable part of the plate and that which has begun to subduct. We must therefore think about the relationship between the anisotropic layers in the two environments.

Are we looking at the same phenomenon in both cases, or does the anisotropy in the different areas have a different origin? Does it occur within the same medium? Is the anisotropic layer in the two regions at different depths below the surface of the oceanic plate? Is the anisotropy in the upper mantle of the stable plate conserved on subduction, or does some process of crystal reorganisation take place in response to the new stress field?

Not all of these questions can be answered because the data and techniques described in 
Chapter 6 to measure the variation of $P_{n}$ velocity with azimuth do not allow the depth of the anisotropic layer to be inferred. It is, however, worth investigating the ways in which the physical conditions and changes at a subduction zone may affect the anisotropy of the upper mantle.

A plausible hypothesis is that the existing anisotropy within the Pacific ocean plate is modified and possibly enhanced by the changing stress field encountered by the plate during the initial stages of subduction. Justification of this hypothesis would require knowledge about the following physical conditions and processes: a) the stress distribution within the subducted plate in the region of interest, and the approximate temperature and pressure conditions; b) the behaviour of olivine, already in a state of alignment, in response to a changing stress pattern and changing temperature/pressure conditions.

The knowledge of the stress distribution within the plate beneath the North Island is not very well known at depth. From focal mechanisms of earthquakes within the slab, as described in Chapter 1, it appears that, at least below the Hawkes Bay region, the stress field changes from downdip tension in the upper (convex) part of the slab resulting from slab pull, to downdip compression in the lower (concave) part (Bannister, 1988). This state of stress is consistent with the results of Chapple and Forsyth (1979) about the relation between bending stresses, strain released by earthquakes and the rheology of the plate. In order to best explain both the topography of the outer rise as well as the relative numbers of tensional and compressional earthquakes within subducting slabs, the authors model the plate as a 2-layer elastic - perfectly plastic slab, in which the yield stress of the upper layer is smaller than that of the lower layer. In this model the bending stress with depth varies as shown in Figure 7.4, changing over from downdip tension to downdip compression at a neutral surface around $33 \mathrm{~km}$ deep. The depth of the neutral surface is subject to the amount of regional stress.

The state of stress within the plate in this region of New Zealand may also be affected by the fact that, immediately south of the area of interest, the subduction is interrupted by the presence of the continental-like crust of the Chatham Rise. Another factor that should affect the stress pattern with depth is the right lateral shear component between the Pacific and Australian plates which is evident at the surface. The directions of the principal axes of stress at a given depth within the subducted slab should be a result of these, and other, regional stress patterns.

It is proposed here, that at the depth of $36-50 \mathrm{~km}$ (18 - $32 \mathrm{~km}$ below the slab surface) in which the high velocities are inferred to exist, the downdip compression induces olivine alignment along strike. This depth is consistent with Chapple and Forsyth's estimate of $33 \mathrm{~km}$ for the neutral surface below which, the state of stress reverses to downdip compression. It is also consistent with Bannister's estimate of a 10 - $12 \mathrm{~km}$ thick layer at the top of the subducted slab in which normal faulting due to downdip tension prevails. In his data set chosen for focal mechanism determination, the events within the slab that result from downdip tension have a maximum depth of $33 \mathrm{~km}$. The depth range for anisotropic structure also agrees with the results of Nishimura and Forsyth (1989) who, by anisotropic inversion of surface wave data conclude 


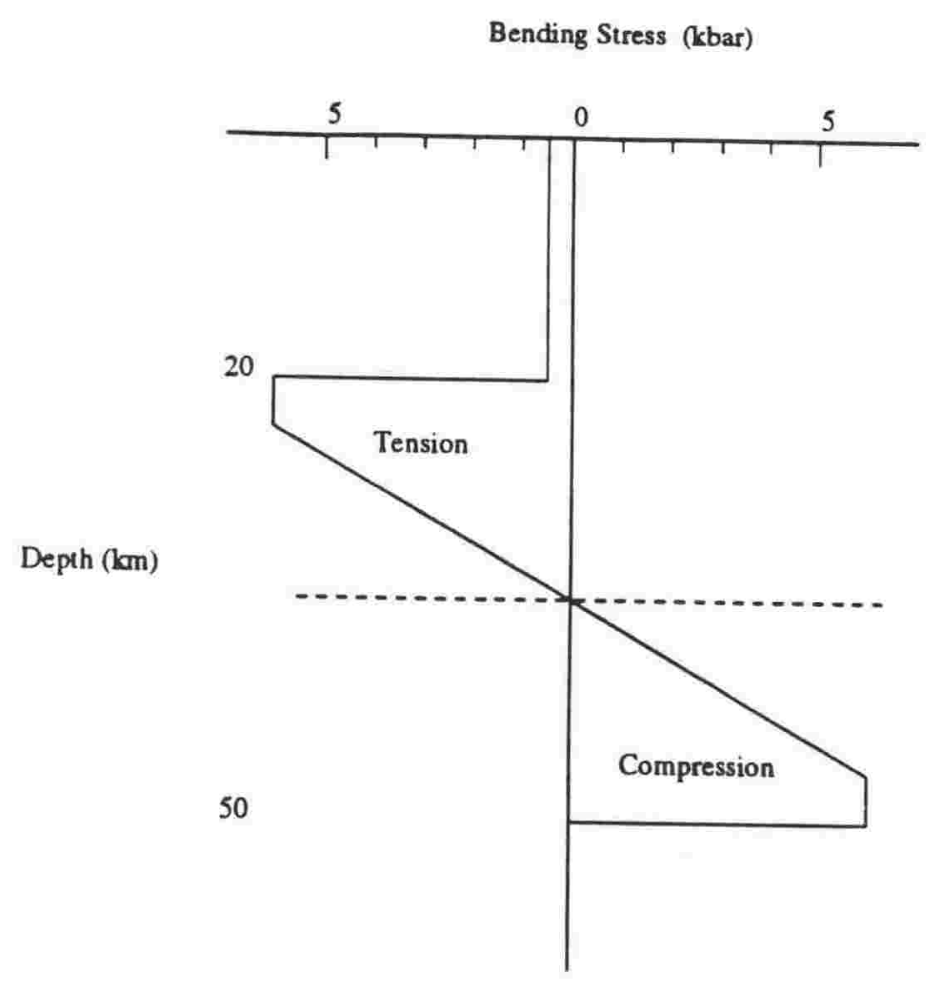

Fig. 7. 4 Bending stress with depth below sea level within a subducting slab (Chapple and Forsyth, 1979). The plot represents a vertical cross section through the slab at approximately $30 \mathrm{~km}$ landward from the trench.

that, in regions of the Pacific older than 80 My (such as the subducting plate below New Zealand), azimuthal anisotropy is confined to depths shallower than $50 \mathrm{~km}$.

The general orientation of the fast P-velocity axis of upper mantle olivine normal to a spreading ridge axis is usually considered to be a "frozen-in" feature, and in fact utilized to infer fossil sea-floor spreading directions. This is probably true for the upper mantle within stable oceanic lithosphere in which the oriented olivine grains drift passively with the plate, but the stress encountered on subduction may produce new effects.

The deformation of olivine and OPX both as single crystals as well as grains within mantle mineral aggregates under various physical conditions has been studied experimentally and even numerically (e. g. Ave'Lallement and Carter, 1970; Carter and Ave'Lallement, 1970; Carter et al, 1972; Durham and Goetze, 1977; Wenk et al, 1991; Bai and Kohlstedt, 1992). The knowledge of such deformation is important in the study of the rheological behaviour of the upper mantle. The experiments of Ave'Lallement and Carter, in particular, have concentrated on the behaviour 
of olivine grains in naturally occurring dunite samples under a range of conditions of temperature, pressure and strain rate. They showed that, above a temperature of about $500^{\circ} \mathrm{C}$ recrystallization of dunite takes place by formation of new grains whose orientation is controlled by the stress field. New grain boundaries advance into old ones until the whole medium consists of grains that are favourably oriented with respect to the stress. The favoured orientation is with the minimum velocity (010) axis parallel to $\sigma_{1}$, the direction of maximum compression. At low rates of deformation (in the order of $10^{-14}$, as expected in nature), this process may take place at temperatures of about $500^{\circ} \mathrm{C}$ and over. The authors claim that syntectonic recrystallization may dominate over plastic flow as a mode of deformation. Fuchs (1987), from studying $P_{n}$ anisotropy in continental lithosphere, notes that the olivine alignment is a recent process, responding to crustal shear. Relationships between anisotropy directions and the present crustal stress field have been found in Southern Germany.

Experiments concentrating on the plastic flow mechanism of olivine also yield similar results ( e.g. Babuska, 1981; Nicolas and Christensen, 1987). The numerical simulations of the plastic flow of peridotite (Wenk et al, 1991) predict olivine (010) (slow) axes aligning with the direction of shortening, normal to the compression axis (Figure 7.5).

Other experiments have also shown that the alignment of olivine and OPX is facilitated by an increase in the ductility of the material, which in turn depends on temperature and pressure conditions as well as on the presence of water. Dunite at low temperatures is difficult to deform, but at high temperatures it flows easily (Chapple and Forsyth, 1979). Bean and Jacob (1991) propose that the formation of anisotropic layers within the lithosphere (including continental lithosphere) is a result of a "shear heating" mechanism. They argue that shear stresses in the lithosphere generate enough heat which, after leaking into surrounding material, increases its ductility and its susceptibility to preferred crystalline alignment by the ambient stress field.

Within a subducting plate, Chapple and Forsyth (1979) note that mechanisms of earthquakes in the 40 - $50 \mathrm{~km}$ depth range within subducted slabs are difficult to explain in terms of frictional sliding on a fault, according to their rheological model of the bending plate. In this depth range the maximum compressive stress is almost horizontal and the minimum compressive stress is approximately vertical and equal to about $15 \mathrm{kbar}$ (the overburden pressure). This would require , they argue, stress differences of about $34 \mathrm{kbar}$ to cause failure, and this is difficult to achieve with their model. They thus propose that the most likely mechanism in this depth range is "some sort of material instability in the plastic regime, a zone of concentrated strain softening". This mechanism is referred to as "ductile faulting" by Post (1977). It is interesting to observe that this depth coincides well with the depth of the high velocity material found in this study, hinting at the possibility of a correlation between this ductility and P-wave anisotropy.

The conditions of temperature, pressure and water content with depth within the subducted slab below New Zealand are less well known. The temperatures in old oceanic lithosphere at depths of around $40 \mathrm{~km}$ would lie in the range $400-600^{\circ} \mathrm{C}$ (Chapple and Forsyth, 1979; Hales, 


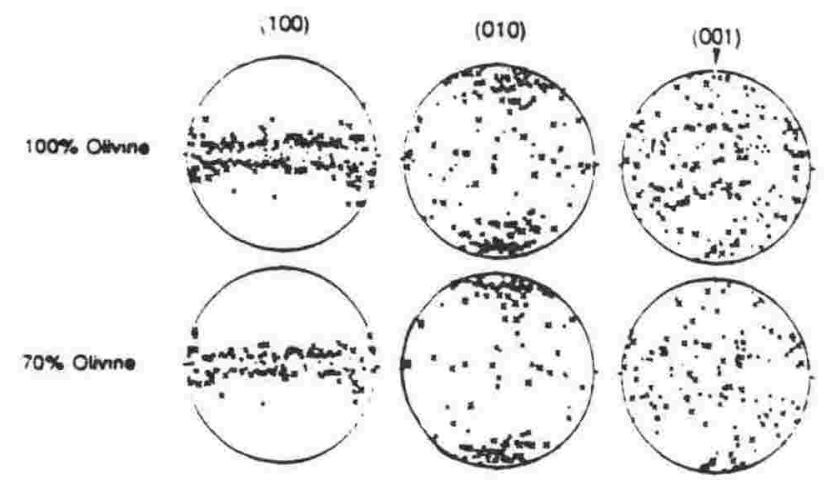

Fig.7.5 Results of numerical simulation of the orientation of olivine grains under axial compression along the 001 axis (Wenk et al, 1991)

1991). Moreover, Bean and Jacob (1991) estimate that a relative velocity of $2 \mathrm{~cm} /$ year between sliding blocks would result in a temperature rise of about $250^{\circ} \mathrm{C}$ at $45 \mathrm{~km}$ depth, a condition that would apply at a subduction zone.

The formation of anisotropic layers within the upper mantle need not then be confined to the regions of mid-ocean ridges, and the "frozen-in" state within the oceanic upper mantle, but may be a more dynamic phenomenon, responding to a changing stress pattern such as that within a subduction zone. It is proposed here that the combination of stress, temperature and pressure conditions at about 35 - $50 \mathrm{~km}$ depth is favourable for the realignment of olivine crystals within the plane of the Pacific upper mantle along strike of the plate in response to the downdip compression. The discrepancy between the magnitude of $\left(\mathrm{V}_{\mathrm{P}}\right)_{\max }(8.37 \mathrm{~km} / \mathrm{s})$ in the stable Pacific plate and that in the downgoing slab $(8.75 \mathrm{~km} / \mathrm{s})$ may be tentatively explained by an enhancement in the alignment of olivine grains as a result of the change in stress field, and/or by the increase in pressure. The overburden pressure alone on a subducting plate at about $30 \mathrm{~km}$ below the surface is calculated to be about $10 \mathrm{kbar}$ (Chapple and Forsyth, 1979). In the 
experiments of Christensen and Ramananantoandro (1971) on samples of dunite, the P-velocity along the fast axis in one sample increased from $8.568 \mathrm{~km} / \mathrm{s}$ at $1 \mathrm{kbar}$ to $8.762 \mathrm{~km} / \mathrm{s}$ at $10 \mathrm{kbar}$, and in another sample from $8.939 \mathrm{~km} / \mathrm{s}$ to $9.150 \mathrm{~km} / \mathrm{s}$ at the same pressures. Thus, velocities of $8.75 \mathrm{~km} / \mathrm{s}$ within oceanic upper mantle at a depth of $36 \mathrm{~km}$ are not unlikely to occur.

\subsection{Discussion of the velocity profile}

The modelling of whole waveforms carried out in this study has not been exhaustive, and there is scope for much wider and deeper investigation, even with the same data. However, it has been shown from the results that this method can be a powerful tool for revealing some special velocity structures. With the right data, and with complementary techniques, such as 2 and 3-dimensional ray tracing, it provides extra constraints on structures.

The focus of waveform modelling in this study has been on the subducted upper mantle structure, and on the presence, or otherwise, of a low velocity layer at the top of the subducted plate. Although the modelling did not strictly distinguish between an $8.75 \mathrm{~km} / \mathrm{s}$ half space and an $8.75 \mathrm{~km} / \mathrm{s}$ layer, the presence of a layer is assumed. This is reasonable when we consider that the pre-existing anisotropy in the Pacific plate is confined to a layer, and that the stress, temperature and pressure conditions that favour enhanced, along-strike olivine alignment within the slab probably only occur within a limited depth range.

The process of wave propagation within the "thin" high velocity layer has not been mathematically treated. From the modelling carried out, it appears that the $P_{n}$ pulse and high frequency $P_{h f}$ phase can be adequately explained for this data set in terms of propagation through a layered structure around the $8.75 \mathrm{~km} / \mathrm{s}$ layer. A problem with the data is that the epicentral distance is too small for enough separation of the initial phases to be observed, and also that it represents only one point in the propagation. The long-range synthetic profile (Figure 5.7) shows the high frequency phase to have an apparent velocity of approximately $8.0 \mathrm{~km} / \mathrm{s}$. This apparent velocity could not be confirmed from the data as was done for $P_{n}$ because the coherency of the signal across the array broke down too rapidly after the first arrival for the purpose of measuring very small differences in arrival time. If a long range data set is available with these phases evident, then such a measurement would be useful for confirming the mode of propagation. A high-velocity layer would be an inefficient waveguide because energy is refracted out of the layer, as opposed to a low-velocity layer. This is consistent with the result that the phase following $P_{n}$ is associated with the overlying layers rather than with the high velocity layer itself.

Sereno and Orcutt (1985) have studied oceanic $P_{n}$ phases in the Pacific Ocean, which consist of a high frequency, low attenuation wavetrain of long duration with a velocity near $8.0 \mathrm{~km} / \mathrm{s}$. By generating synthetic seismograms they concluded that this phase could be explained simply as a 
set of refractions from the lower lithosphere. The long duration in the ocean phase was explained as due to reverberations in the ocean column. They argued that the propagation of the high frequency $P_{n}$ does not require special features of the lithosphere, not even high $Q$ values. This agrees with the interpretation of $\mathrm{P}_{\mathrm{hf}}$ in Chapter 5 .

There are similarities and differences between this study's interpretation of the mantle phases and that of Gubbins and Snieder (1990) regarding the high frequency precursors. In their study of the subducted Pacific plate, these authors also conclude that any high velocity layer cannot be thinner than $6 \mathrm{~km}$, otherwise it would not retain energy. This is similar to the results of Section 5.6.3, in which the thickness of the layer was inferred to be greater than $4 \mathrm{~km}$. Their waveforms showed a high frequency precursor arriving before a low frequency phase, the latter corresponding to "normal" upper mantle velocity. They interpreted this as a dispersed wave signal through the structure consisting of a high velocity layer bounded by velocity gradients and/or finer layering. This is also similar to the present interpretation of structure, however it is unjustified to compare the two waveforms, because the distance of propagation is on a totally different scale.The petrological interpretation of the high velocities is different, but Gubbins and Snieder's wavepaths sample greater depths, hence an eclogite interpretation in their case is reasonable.

The results of this study are also very similar to those of Ouchi et al (1983), who studied the high frequency $P_{n}$ and $S_{n}$ phases in the Pacific. These phases were previously studied by Walker (1977), who proposed the presence of one, or two, waveguides capable of transmitting high frequency $P_{n}$ and $S_{n}$ to large distances. Ouchi et al recorded high frequency $P_{n}$ phases at distances between $6^{\circ}$ and $18^{\circ}$ in the north-western Pacific. These phases consisted of a lower frequency $(3 \mathrm{~Hz})$ mantle refracted phase travelling at $8.4-8.6 \mathrm{~km} / \mathrm{s}$, followed by a higher frequency $(>6 \mathrm{~Hz}$ ) phase travelling at $8.1-8.3 \mathrm{~km} / \mathrm{s}$. The high frequency phase had a long duration and small attenuation with distance. These phases are analogous to the $P_{n}$ and $P_{h f}$ phases observed in this study.

\subsection{Unanswered questions and suggestions for further research}

This study has touched upon several features of the subducted slab structure that merit further investigation.

1. In Chapter 3, the determination of the value of the refractor velocity through 3-dimensional ray trace modelling has been shown to be sensitive to local variations in the form of curvature of the slab. It is worth considering in more detail the extent to which this is influencing the measurements of $P_{n}$ and $S_{n}$ velocities in the North Island. Purposely designed 
array measurements of wavefront characteristics, in conjunction with 3-dimensional ray tracing, might conversely yield information about the finer details of local slab geometry.

2. The high velocity layer inferred in this study has been assumed to represent the fast direction of an anisotropic medium, but the existence of anisotropy within the slab has not heen demonstrated. Shear wave polarization analysis and reflectivity modelling incorporating anisotropic media may supply further evidence, provided the effects are distinguished from those due to anisotropy in the overlying crust (Gledhill, 1991). In the Weber data set, although shear wave splitting was not specifically studied, some evidence of its existence was seen in a few seismograms, although its origin cannot be ascertained. One example is shown in Figure 7.6, in which the radial and transverse components of the horizontal motion show that the corresponding S-waves are separated by about $0.3 \mathrm{~s}$. Jacob et al (1991) have modelled anisotropic layers within the lithosphere by generating seismic sections at varying azimuths. The appearance of such sections is highly sensitive to the direction of propagation. Such techniques, however, may be difficult to apply in the case of New Zealand, where the 3-dimensional structures make it difficult to interpret sections at different azimuths.

$\mathrm{P}$-wave anisotropy within the subducted upper mantle is expected to have an effect on the Pwave polarization anomaly, due to deviation of the seismic ray paths from the vertical plane connecting source and receiver (in the case of horizontal layering). Thus the azimuthal anomaly at the station is a sum of this effect as well as of slab geometry, although the anisotropy effect is expected to be smaller than the geometry effect (Shearer and Orcutt, 1985; de Parsceau, 1991). This effect should be investigated.

More complete determination of the anisotropy within the subducting lithosphere may contribute to the knowledge of the state of stress within the downgoing slab.

3. A more detailed velocity profile in the vicinity of the high velocity medium, as well as more rigorous constraints on attenuation parameters may be investigated, using the reflectivity modelling used here, by utilizing a long-range refraction profile, such as that produced in the Hikurangi Margin refraction experiment. Amplitude - distance variations could also yield information on velocity gradients.

4. The idea of enhancement of a pre-existing anisotropic layer within oceanic upper mantle on encountering the stress conditions within a subduction zone could be further investigated by looking for similar effects in other subduction zones around the world.

5. The S-wavefield has not been investigated in this study. Waveform modelling could be extended to the S-waves, and the horizontal components of displacement, to yield more information about $V_{\mathrm{P}} / \mathrm{V}_{\mathrm{S}}$ ratios, as well as $\mathrm{Q}_{\mathrm{P}}^{-1} / \mathrm{Q}_{\mathrm{S}}^{-1}$ relationships. 

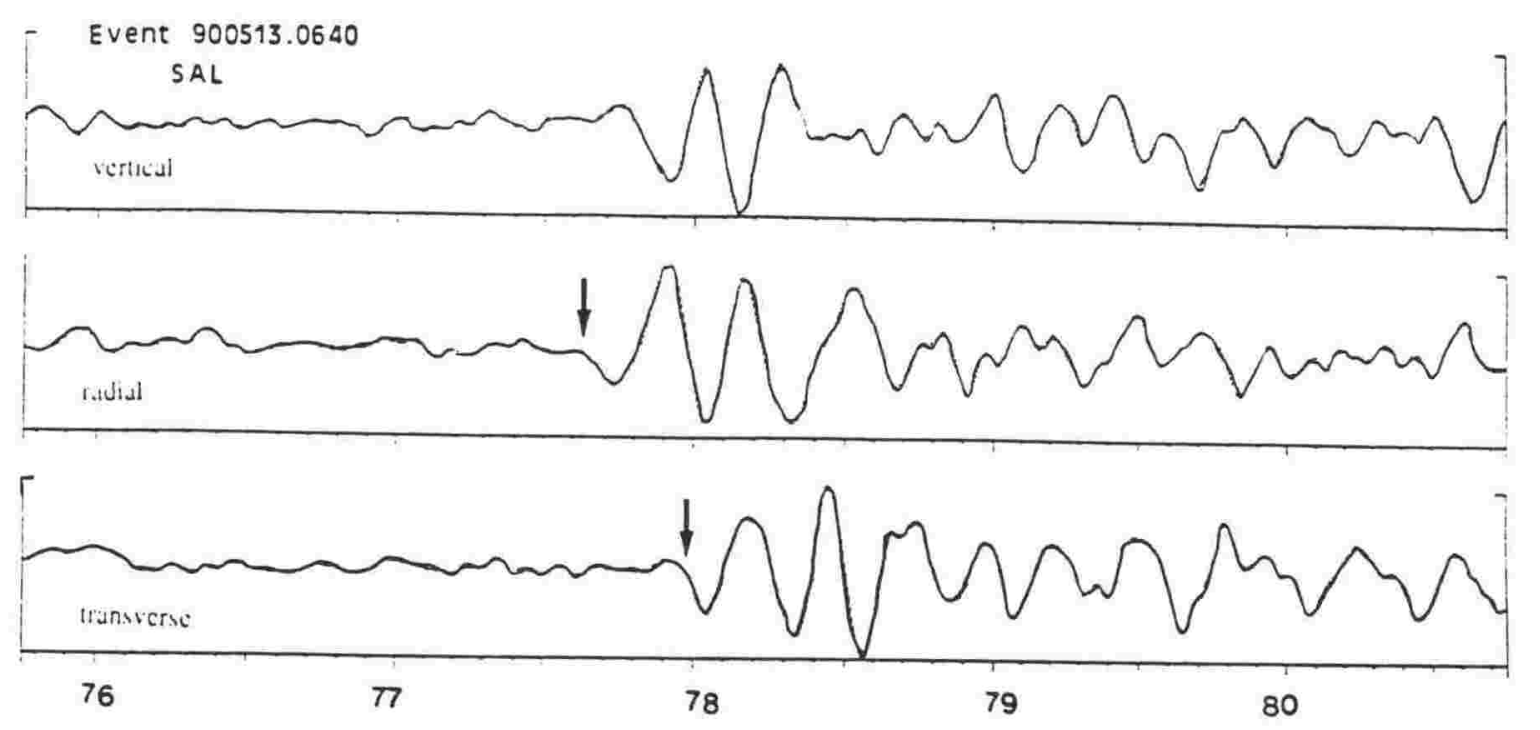

Fig. 7.6 Example of shear wave splitting observed in the Weber data set

6. The effect of the low-velocity layer on top of the subducted slab was one of the clearest results illustrated by the reflectivity modelling. The similarity of the synthetic signal and the character of some East Coast data is strong evidence for the presence of such a layer, and confirms previous reports from independent methods. The question of how far down this layer penetrates along with the slab has not been addressed. Other methods for detecting it may be applied, particularly looking at path effects updip along the slab, using deeper intraplate earthquakes (e. g. Crosson et al, 1994).

7. A competely different problem, entering the domain of the palaeotectonics of the Pacific Ocean, would be to explain the $\mathrm{N} 60^{\circ} \mathrm{E}$ "fast" direction of upper mantle anisotropy in the triangular section of lithosphere studied in Chapter 6. The problem arises when one correlates this direction with the fossil spreading direction. This particular area poses a problem with regard to its origin because it lacks magnetic lineations, presumably having been formed during the Cretaceous Quiet Zone, in which no known reversals of the earth's magnetic polarity occurred. It 
is generally assigned an age of around $100 \mathrm{My}$ (Hilde et al, 1977).

The $\mathrm{N} 60^{\circ} \mathrm{E}$ direction is approximately at right angles to the present direction of motion of the Pacific plate where it subducts below New Zealand. The area in question appears, at first sight, to be continuous with the crust currently being formed at the Pacific-Antarctic Ridge in a general N-W trending direction. This would be inconsistent with the fossil spreading direction inferred by the anisotropy.

The tectonic history of the South Pacific ocean is complex. Hilde et al (1977) propose that the Pacific plate had its origin at a triple junction that existed about $190 \mathrm{My}$ ago between three major plates - the Kula, Phoenix and Farallon plates, which subsequently moved apart, forming an initially triangular shaped new plate (the Pacific plate) between them (Figure 7.7), and three active spreading ridges. The Pacific - Phoenix spreading direction was roughly N - S (Hilde et al, 1977; Larson and Chase, 1972). The east-west trending Phoenix magnetic lineations, mapped just north of the Phoenix islands, date as far back as approximately 120My (Larson and Chase, 1977) and are believed to represent this ancient spreading system. As the Pacific plate increased in size, the Kula plate was subducted in the North below the Asian plate (Uyeda and Miyashiro, 1974), and at the same time, the Phoenix - Pacific - Farallon triple junction migrated rapidly south-eastward. The Phoenix plate is presumed to have subducted below the Gondwana margin at a time when New Zealand was still joined to Antarctica. The breaking away of New Zealand (including the Chatham Rise and Campbell Plateau) from Antarctica is believed to have occurred 81 My ago (Molnar et al, 1975).

The present day tectonics of the South Pacific (Figure 7.8 ) are largely dominated by the Pacific - Antarctic Ridge, where sea-floor spreading is causing the Pacific plate to move in a general NW - SE trend at a rate of 6 to $10 \mathrm{~cm} / \mathrm{yr}$ (Molnar et al, 1975), with spreading taking place along large scale fracture zones, such as the Eltanin. Heezen and Tharp fracture zones (Watts and Weissel, 1988). Magnetic lineations on both sides of the ridge date back to $81 \mathrm{My}$. It is not unequivocally clear what the origin of the part of the Pacific plate presently subducting below New Zealand is.

An interesting development has been reported recently by Engebretson et al (1991). These authors have discovered weak magnetic lineations south of the Phoenix islands $\left(3^{\circ} \mathrm{S}, 175^{\circ} \mathrm{W}\right)$, becoming progressively older to the south-east. These lineations, which they name the Tonga lineations, are believed to be as old as $125-150 \mathrm{My}$. If this is the case, they argue, then the crust east of the Tonga trench may be older than the Cretaceous Quiet Zone, and may even be a remnant of the old Phoenix plate that got "trapped" with the Pacific plate during a period of plate reorganization. This would be more consistent with a fossil spreading direction as indicated by the anisotropy results of this study, as well as those of Shearer and Orcutt (1985).

Further discussion of this topic here is unjustified and beyond the scope of this thesis, as it requires far more detailed information and elaboration on the tectonic history of the Pacific Ocean. It would be interesting and important, however, if the presence of upper mantle 

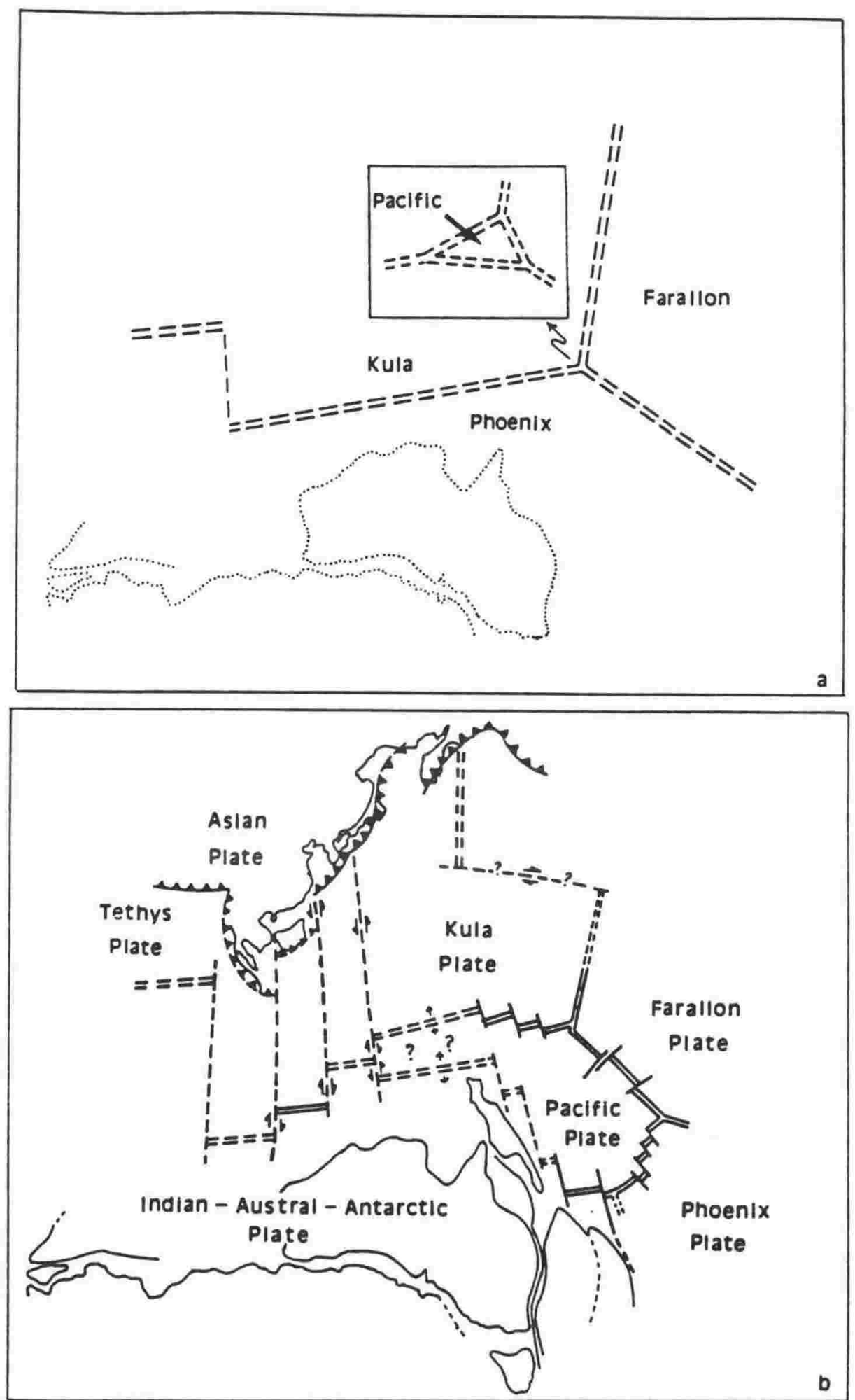

Fig. 7.7 a)Creation of the Pacific plate 190 My ago

b)The situation 135 My ago (Hilde et al, 1977) 


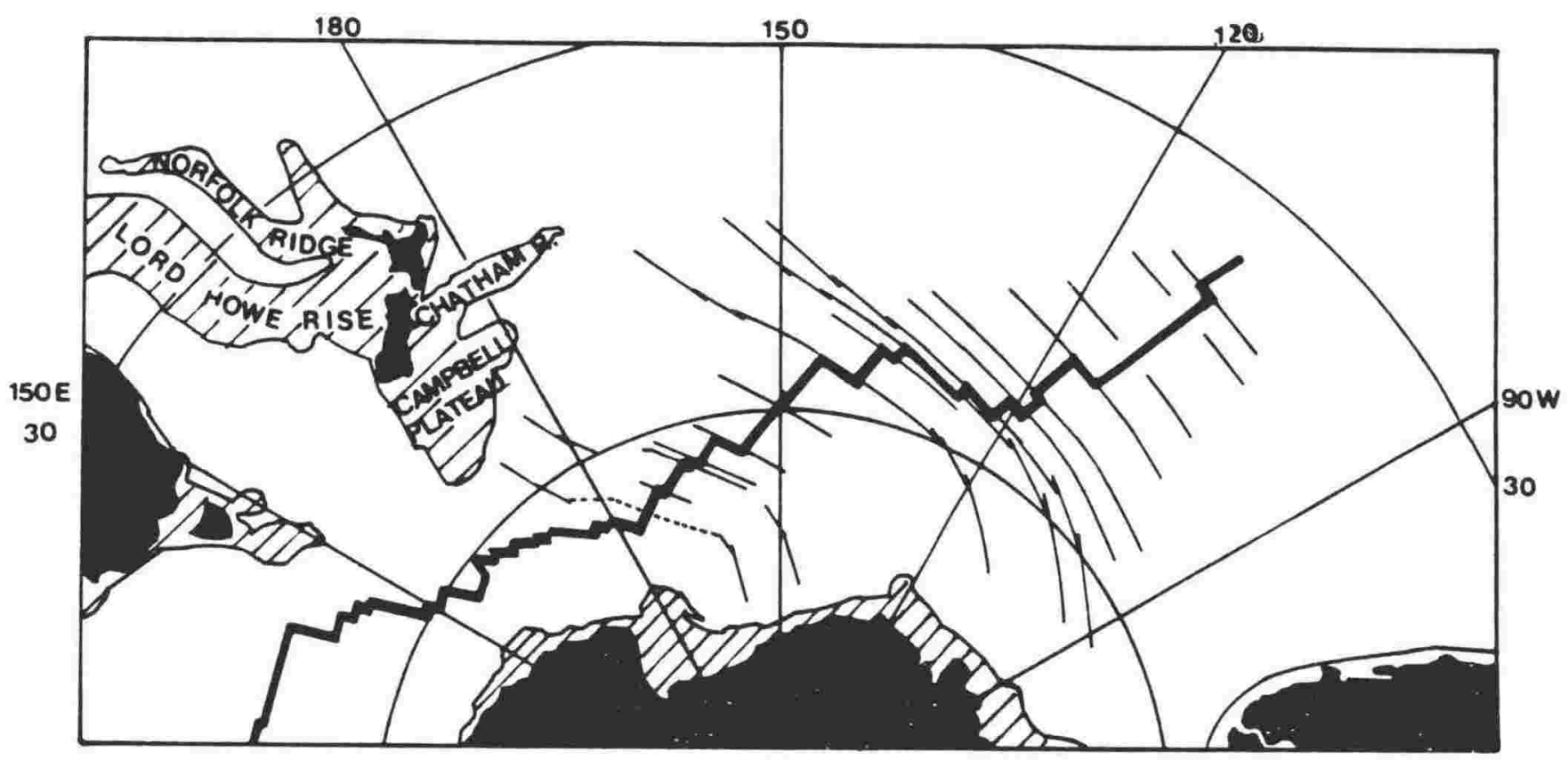

Fig. 7.8 Present day tectonics in the South Pacific (Molnar et al, 1975)

anisotropy could help in some way unravel the complexities of this fragment of the South Pacific Ocean. 


\section{REFERENCES}

Adams, R. D. (1962). Thickness of the earth's crust beneath the Campbell Plateau , N.Z J. Geol. Geophys., 5, $74-85$.

Adams, R. D. and Ware, D. E. (1977). Subcrustal earthquakes beneath New Zealand; locations determined with a laterally inhomogeneous velocity model, N.Z.J.Geol.Geophys., 20, $59-83$.

Aggarwal, Y. P., Barazangi, M., and Isacks, B. (1972). P and S travel times in the Tonga-Fiji region: a zone of low velocity in the uppermost mantle behind the Tonga island arc, J. Geophys. Res., 77, 6427 . 6433 .

Anderson, D. L. (1979). The upper mantle transition: eclogite?, Geophys. Res. Lett., 6, 433 - 436.

Ansell, J. H. (1978). The location and mislocation of subcrustal earthquakes, Phys. Earth Plan. Int., 17, P1-P6.

Ansell, J. H. and Gubbins, D. (1986). Anomalous high-frequency wave propagation from the TongaKernadec seismic zone to New Zealand, Geophys. J. R. astr. Soc., 85, 93- 106.

Ansell, J. H. and Bannister, S. (1991). Morphology of the shallow subducted slab (Abstract), Geophysical Symposium, N.Z. Geophys. Soc., Wairaki, New Zealand,

Asada, T. and Shimaunura, H. (1976). Observation of earthquakes and explosions at the bottom of the Western Pacific : structure of oceanic lithosphere revealed by Longshot experiment, in:, The Geophysics of the Pacific Basin und its Margin, Am. Geophys. Union Monograph, 19, 135 - 154.

Asada, T., Shimaunura, H., Azano, S., Kobayashi, K., and Yoshibumi, T. (1983). Explosion seismological experiments on long-riunge protiles in the Northwestern Pacific and the Marianas sea, in:, Geodynamics of the Western Pacific-Indonesian region, Geodynamic Series, 11, T. Hilde \& S. Uyeda eds., A.G.U., Washington.

Ave'Lallement, H. G. and Carter, N. L. (1970). Syntectonic recrystallization of olivine and modes of flow in the upper mantle, Geol. Soc. Am. Bull., 81, $2203-2220$.

Babuska, V. (1981). Anisotropy of $v_{p}$ and $v_{s}$ in rock-torming minerals, J. Geophys., 50, 1 - 6.

Backus, G. E. (1965). Possible forms of seismic anisotropy of the uppermost mantle under oceans, $J$. Geophys. Res., 7), 3429 - 3439.

Bai, Q. and Kohlstedt, D. L. (1992). High-temperature creep of olivine single crystals, 2. dislocation structures, Tectonophysics, 206, 1 - 29.

Baker, D. W. and Carter, N. L. (1972). Seismic velocity anisotropy calculated for ultrarnafic minerals and aggregates, Geophys. Monogr. Ser., 16, Heard, H. C. ed., A.G.U., Washington, D.C..

Bannister, S. (1988). Microseismicity and velocity structure in the Hawkes Bay Region, New Zealand, Geophys. J., 95, 45 - 62 .

Bock, G. (1981). The effect of the descending lithosphere on the Tonga island arc on P-wave travel-time residuals at the Wirriununga seismic auray, Phys. Earth Plan. Int., 25, $360-371$.

Bean, C. J. and Jacob, A. W. B. (19)1). P-wave anisotropy in the lower lithosphere, Earth. Planet. Sci. Lett., 99, $58-65$.

Blomer, S. H. and Fisher, R. L. (1987). Petrology and gecochemistry of igneous rocks from the Tonga trench - a non-accreting plate boundary, J. Geology, 95, 469 - 495.

Boudier, F. and Nicolas, A. (1986). The harzburgite and the therzolite subtypes in ophiolitic and oceanic environunents, Earth. Plan. Sci. Lett., 76, 84 - 92.

Buchbinder, G. G. R. and Haddon. R. A. W. (1990). Azimuthal anomalies of short-period P-wave arrivals from Nahanni attershocks, Northwest Territories, Canada, and effects of surface topography, Bull. Seism. Sic. Ann., 80, $1272-1283$. 
Burton, P. W. and Blamey, C. (1972). A computer program to determine the spectrum and dispersion characteristics of a transient seismic signal, A.W.R.E. Rep. No. (0/48/72, H.M.S.O., London.

Carter, N. L. and Ave'Lallement, H. G. (1970). High temperature How of dunite and peridotite, Geol. Soc. Am. Bull., 81, 2181 - 2202.

Carter, N. L., Baker, D. W., and George, R. P. (1972). Seismic anisotropy, flow and constitution of the upper mantle, in:, Flow and Fracture of Rocks, Geophys. Monogr. Ser, 16, Heard, H. C. ed., A.G.U., Washington, D.C..

Cerveny, V., Klimes, L., and Psencik, I. (1988). Complete seismic ray tracing in three-dimensional structures, in:, Seismological Algorithms, D. Dommbos ed., Academic Press, London.

Chadwick. M. (1990). Attenuation and source parameters of small earthquakes recorded in the Wellington region, New Zealand, M.Sc. Thesis, Victoria University of Wellington, New Zealand.

Chadwick. M. and Reyners, M. (1992). Structure along the shallow part of a subduction zone: first results from the Palliser Bay - Hawkes Bay refraction experiment, New Zealand (abstract), EOS Transactions (1992 Fall Meeting Supplement), 73, A.G.U., Washington, U.S.A..

Chapple, W. and Forsyth, D. (1979)). Earthquakes and bending of plates at trenches, J. Geophys. Res., 84, $6729-6749$.

Chong, F. K. L. (1982). A microe:urthyuake study of the Indian/Pacitic boundary, Central Hawkes Bay, New Zeadand, Ph.D. Thesis, Victoria University of Wellington, New Zealand.

Christensen. N. I. and Ramananantoandro, R. (1971). Elastic moduli and anisotropy of dunite to 10 kilobars, J. Geophys. Res., 17, 4(0)3 - 4010 .

Christensen, N. I. and Salisbury, M. H. (1979). Seismic anisotropy in the oceanic upper mantle: evidence from the Bay of Islands ophiolite complex, J. Geophys. Res., 84, 4601 - 4610.

Christoffel, D. A. and Falconer, R. K. H. (1972). Marine magnetic measurements in the south-west Pacific ocean and the identification of new tectonic features, Antart. Res. Ser., 19, 197 - 209.

Cole, J. W. and Lewis, K. B. (1981). Evolution of the Taupo-Hikurangi subduction system, Tectonophysics, 72. $1-21$.

Coleman, R. G. (1971). Plate tectonic emplacement of upper mantle peridotites along continental edges, $J$. Geophys. Res., 76, $1212-1222$.

Crampin, S. and Bamford, D. (1977). Inversion of P-wave velocity anisotropy, Geophys. J. R. astr. Soc., 49. $123-132$.

Crosson, R. S. and Christensen, N. I. (1969). Transverse anisotropy of the upper mantle in the vicinity of Pacitic tracture zones, Bull. seism. soc. Am., 59, 59- 72.

Davey, F. J. and Smith, E. G. C. (1983). A crustal seismic reflection-refraction experiment across the subducted Pacific plate under Wellington, New Zealand, Phys. Earth Plan. Int., 31, 327 - 333.

Davey, F. J., Hampton, M., Childs, J., Fisher, M. A., Lewis, K., and Pettinga, J. R. (1986). Structure of a growing accretionary prism, Geology, 14, $663-666$.

Den, N., Ludwig, W. J., Murauchi, S., Ewing, J. 1., Hotta, H., Edger, N. T., Yoshii, T., Asanuma, T., Hagiwara. K., Sato, T., and Ando, S. (1969). Seismic retraction measurements in the north-west Pacitic basin, J. Geophys. Res., $1421-1434$.

Dibble, R. R. (1957). The apparent velocity of $\mathrm{Pn}$ and $\mathrm{Sn}$ between New Zealand and the Kermadec Islands (abstract), Australian and New Zealand Association for the Advancement of Science, Dunedin Meeting, 16-23 Jan. 1957, Paper C46,

Durham, W. B. and Givetze, C. (1977). Plastic flow of oriented single crystals of olivine, 1, Mechanical data, J. Geophys. Res., 82, 5737 - 5754.

Dziewonski, A. M., Bloch, S., and Landisman, M. (1969). A technique for the analysis of transient seismic signals, Bull. Seism. Soc. Am., 59, $427-444$. 
Engebretson, D. C., Mammerickx, J., and Raymond, C. A. (1991). Tonga Lineations - the Phoenix plate has arisen? (abstract), A.G.U. 1991 Fall Meeting Abstracts, 444.

Falconer, R. K. H. (1972). The Indian - Antarctic - Pacific Triple Junction, Earth. Plan. Sci. Lett., 17, 151 $-158$.

Forsyth, D. W. (1977). The evolution of the upper mantle beneath mid-ocean ridges, Tectonophysics, 38, 89) -118

Fuchs, K. and Muller, G. (1971). Computation of synthetic seismograms with the reflectivity method and comparison with observations, Geophys. J. R. astr. Soc., 23, 417 - 433.

Fuchs, K. and Schultz, K. (1976). Tunnelling of low-frequency waves through the subcrustal lithosphere, $J$. Geophys., 42, $175-190$.

Fuchs, K. (1977). Seismic anisotropy of the subcrustal lithosphere as evidence for dynamical processes in the upper mantle, Geophys. J. R. astr. Soc., 49, $167-179$.

Fuchs, K., Vinnik, L. P., and Prodehl, C. (1987). Exploring heterogeneities of the continental mantle by high resolution sesinic experiments, in:, Composition, Structure and Dynamics of the LithosphereAstenosphere System, Geodynamics Series, 16, A.G.U., Washington, U.S.A..

Garrick, R. A. (1968). A reinterpretation of the Wellington crustal refraction profile, N.Z. J. Geol. Geophys. , 11, $1280 \cdot 1294$.

Gledhill, K. R., Randall, M. J. , and Chadwick, M. P. (1991). The EARSS Digital Seismograph: System Description and Field Trials, Bull. Seism. Soc. Am., 81, 1380 - 1390.

Gledhill, K. R. (1991). Sheir wave splitting and seismic anisotropy in the Wellington region, New Zealand, Ph.D. Thesis, Victoria University of Wellington, New Zealand.

Gubbins, D. and Snieder, R. (1991). Dispersion of P waves in subducted lithosphere: evidence for an eckogite layer, J. Geophys. Res. , 96, $6321-6333$.

Haines, A. J. (1976). Compressional and shear velocities in the upper mantle beneath New Zealand determined using local crustal earthquakes, M.Sc. Thesis, Victoria University of Wellingion, New Zeadand.

Haines, A. J. (1979). Seismic wave velocities in the uppermost mante beneath New Zealand, N.Z J. Geol. Geophys., 22, $245-257$.

Hades, A. L., Helsely, C. E., ind Nation, J. B. (1970). P travel times for an oceanic path, J. Geophys. Res., $75,7362 \cdot 7381$.

Hales, A. L. (1991). Upper mantle inodels and the thickness of the continental lithosphere, Geophys. J. Int., 105, $355 \cdot 363$.

Hess, H. H. (1964). Seismic anisotropy of the upper mantle under oceans, Nature, 2103, $629-631$.

Hilde, T. W. C., Uyeda, S., and Kroenke, L. (1977). Evolution of the western Pacific and its margin, Tectonophysics, 38, $145-165$.

Hori, S. (1990). Seismic waves guided by untransformed oceanic crust subducting into the mantle: the case of the Kanto district, central Japan, Tectonophysics, 176, 355 - 376.

Hsu, K. J. (1987). The geology of (אean floor, in:, Composition, Structure and Dynamics of the Lithosphere-Astenosphere System, Geodynamics Series, 16, 291 - 300.

Jacob, A. W. B., Bean, C. J., Nolte, B., and Prodehl, C. (1991). P-wave sections in a realistic anisotropic lithosphere, Geophys. J. Int., 107, 709) - 714.

Kayal, J. R. (1983). A microxearthquake study in a subduction zone: southeast Wellington Province, New Zealind, Ph.D. thesis, Victoria University of Wellington, New Zealand.

Kayal, J. R. and Sinith, E. (1984). Upper mantle P-wave velocities in the southeast North Island, New Zeadiand, Tectonophysics, 1104, 115 - 125. 
Kayal, J. R. (1986). Analysis of strong phases other than $P$ and $S$ from a microearthquake survey in the Wellington region. New Zealand, Bull. seism. soc. Am., 76, 1347 - 1354.

Kenneth, B. L. N. (1983). Seismic wave propagation in stratified media, Cambridge University Press, London.

Kenneth, B. L. N. (1988). Systematic approximations to the seismic wavefield, in:, Seismological Algorithms, D. Doxombos ed., Academic Press, London.

Kind, R. (1976). Computation of reflection coefficients for layered media, J. Geophys., 42, 191 - 200.

Kind, R. (1978). The reflectivity method for a buried source, J. Geophys., 44, 603-612.

Kind, R. (1979). Observations of sPn from Swabian Alb earthquakes at the GRF array, J. Geophys., 45, $337-340$.

Langston, C. A. and Helmberger, D. V. (1974). Interpretation of body and Rayleigh waves from NTS to Tucson, Bull. Seism. Soc. Am., 64, 1919-1929).

Langston, C. A. (1977). The effect of planar dipping structure on source and receiver responses for constant ray parameter, Bull. Seism. Soc. Am., 67, $1029-1050$.

Langston, C. A. (1982). Aspects of $\mathrm{Pn}$ and $\mathrm{Pg}$ at regional distances, Bull. Seism. Soc. Am., 72, 457 - 471.

Larson, R. L. and Chase, C. G. (1972). Late Mesozoic evolution of the Western Pacific Ocean, Bull. Geol. Soc. Am., 83, 3627 - 3644.

Lawson, C. L. and Hanson, R. J. (1974). Solving least squares problems, Prentice-Hall, Inc., New Jersey.

Lee, W. H. K. and Lahr, J. C. (1975). HYPO-71: a computer program for determining hypocenter, magnitude and first motion pattern of local earthyuakes, Open File Report U.S. Geological Survey,

Lewis, K. B. and Davey, F. J. (1987). Environment of a classical accretionary prism, Hikurangi convergent margin, New Zealand, Proceedings, Pacific Rim Congress 87, $263-266$.

Luo, X. (1992). Subduction interface and crustal structure in the Cape Palliser region, North Island, New Zeadand, from observations of Cape Palliser earthquakes, N. Z. J. Geol. Geophys., 35, 447 - 455.

Mitronovas, W., Isacks, B., and Seeher, L. (1969). Earthquake locations and seismic wave propagation in the upper $250 \mathrm{~km}$ of the Tonga island arc, Bull. Seism. Soc. Am., 59, $1115-1135$.

Mitronovas, W. and Isacks, B. (1971). Seismic velocity anomalies in the upper mantle beneath the TongaKermadec island arc, J. Geophys. Res., 76, 7154 - 7180.

Molnar, P., Atwater, T., Marnmerickx, J., and Smith, S. M. (1975). Magnetic anomalies, bathymetry and the tectonic evolution of the South Pacitic since the Late Cretaceous, Geophys. J. R. astr. Soc., 40, $383-420$.

Montagner. J. and Anderson, D. L. (1989). Constrained reference mantle model, Phys. Earth Planet. Int., 58. $205-227$.

Montagner, J. and Tanimoto, T. (1991). Gilobal upper mantle tomography of seismic velocities and anisotropies, J. Geophys. Res., 96, 20337 - 20351.

Morris, G. B., Raitt, R. W., and Shor,Jr., G. G. (1969)). Velocity anisotropy and delay-time maps of the mantle near Hawaii, J. Geophys. Res., 74, 4300-4316.

Muller, G. (1985). The reflectivity method: a tutorial, J. Geophys., 58, 153 - 174.

Mundal, I., Ukawa, M., and Crosson, R. S. (1994). Normal and anomalous phases from local earthquakes, and slab structure of the Cascaddia subduction zone, , (paper in manuscript form).

Niazi, M. (1966). Corrections to apparent azimuths and travel-time gradients for a dipping Mohorovicic discontinuity, Bull. seism. soc. Am., 56, 491-50\%.

Nicolas, A., Boudier, F., and Bouchez, J. L. (1980)). Interpretation of peridotite structures from ophiolitic and (xceanic environments, Am. J. Sci., 280, $192-210$. 
Nicolas, A. and Christensen, N. I. (1987). Formation of anisotropy in upper mantle peridotites - a review, in:, Composition, Structure and Dynamics of the Lithosphere-Astenosphere System, Geodynamice Series, 16, A.G.U., Washington, U.S.A..

Nishimura, C. E. and Forsyth, D. W. (1989). The anisotropic structure of the upper mantle in the Pacific, Geophys. J., 96, $203-229$.

Olsen, K. H., Braile, L. W., and Stewart, J. N. (1983). Modeling short-period crustal phases (P, Lg) for long-range refraction profiles, Phys. Earth Plan. Int., 31, 334 - 347.

Otsuka, M. (1966 (a)). Azimuth and slowness anomalies of seismic waves measured on the central California seismographic array. Part I: Observations, Bull. seism. soc. Am., 56, 223 - 239.

Otsuka, M. (1966 (b)). Azimuth and slowness anomalies of seismic waves measured on the central California seismographic array. Part II: Interpretation, Bull. seism. soc. Am., 56, 655 - 675.

Ouchi, T., Nagumo, S., Kasahara, J., and Koresawa, S. (1983). Separation of high-frequency Pn phases and mantle refracted $P$ phases at distances between $6^{\circ}$ and $18^{\circ}$ in the western Pacific by ocean bottom seismograph array, Geophys. Res. Lett., 10, $1069-1072$.

Pedersen, H. and Ciunpillo, M. (1\%)1). Depth dependence of Q beneath the Baltic Shield inferred from modelling of short period seismograms, Geophys. Res. Lett., 18, $1755-1758$.

Post, R. L. (1977). High-temperature creep of Mt. Burnett dunite, Tectonophysics, 42, 75 - 110.

Prasad, G. and Bock, G. (1987). P.Wave travel times from the Tonga subduction zone to stations on the (xeeanic side of the Tonga-Kermadec trench, Geophys. Res. Lett., 14, 9- 12.

Raitt, R. W., Fisher, R. L., and Mason, R. G. (1955). Tonga Trench, Geol. Soc. America Special Pap., 62, $237-254$.

Raitt, R. W., Shor, G., Morris, G., and Kirk, H. (1969). Anisotropy of the Pacific Upper Mantle, J. Geophys. Res., 74, 3095 - 3109.

Regan, J. and Anderson, D. L. (1984). Anisotropic models of the upper mantle, Phys. Earth Planet. Int. , 35. $227-263$.

Reyners, M. (1980). A micr(xearthyuake study of the plate boundary, North Island, New Zealand, Geophys. J. R. astr. Soc., 63, $1-22$.

Reyners, M. (1983). The Hawke's Bay, New Zealand, earthquake of 1982 September 02 and deformation in the interior of the subducted Pacific plate, Tectonophysics, 106, 259- 273.

Reyners, M. (1989). New Zealand Seismicity 1964-87: an interpretation, N.Z J. Geol. Geophys., 32, 307 315.

Ringwoxd, A. E. (1976). Phase transformations in descending plates: implications for mantle dynamics and differentiation, in: , The Geophysics of the Pacific Basin and its Margin, Am. Geophys. Union Monograph , 19, Sutton, G.H., Manghnani, M.H.,Moberly, R. and McAfee, E.U., eds., 391 - 398.

Ringwood, A. E. (1982). Phase transformations and differentiation in subducted lithosphere: implications for mantle dynamics, basadt petrogenesis and crustal evolution, J. Geol., 90, 611 - 643.

Robinson, R. (1983). Velocity structure of the Wellington region, New Zealand, from local earthquake data and its implications for subduction tectonics, Geophys. J. R. astr. Soc., 75, 335 - 359.

Robinson, R. (1986). Seismicity, structure and tectonics of the Wellington region, New Zealand, Geophys. J. R. a.str. Soc., 87, 379 - 409).

Robinson, R. (1994 (in press)). Shallow subduction tectonics and fault interaction: the Weber, New Zealand, earthquakes of $1990-192$.. J. Geophys. Res.,

Sandmeier, K. J. and Wenzel, F. (1986). Synthetic seismograms for a complex crustal model, Geophys. Res. Lett. , 13, 22 - 25.

Satake, K. and Hashida, T. (1989). Three-dinnensional altenuation structure beneath North Island, New Ze:dind, Tectnophysics, 159, 181 - 194. 
Sereno, T. J. and Orcutt, J. A. (1985). Synthetic seismogram modelling of the oceanic Pn phase, Nature, $316,246-248$.

Shearer, P. and Orcutt, J. (1985). Anisotropy in the oceanic lithosphere - theory and observations from the Ngendei seismic refraction experiment in the south-west Pacific, Geophys. J. R. astr. Soc, 80, 493 . 526.

Shimamura, H. and Asada. T. (1976). Apparent velocity measurements on an oceanic lithosphere, Phys. Earth. Planet. Int., 13, P15 - P22.

Shimamura, H. and Asada, T. (1983). Velocity anisotropy extending over the entire depth of the oceanic lithosphere,in:, Geodynamics of the Western Pacific-Indonesian region, Geodynamic Series, 11, T. Hilde \& S. Uyeda eds., A.G.U., Washington.

Shimamura, H., Asada, T., Suyehiro, K., Yamada, T., and Inatani, H. (1983). Longshot experiments to study velocity anisotropy in the oceanic lithosphere of the northwestern Pacific, Phys. Earth Plan. Int., 31, $348-362$.

Smith, E., Stern, T., and Reyners, M. (1989). Subduction and back-arc activity at the Hikurangi convergent margin, New Zealand, Pageoph, 129, 203 - 231.

Smith, E. (1990). The 1990 Weber, Southern Hawkes Bay, earthyuakes: implications for the seismic hazard in Hawkes Bay, N.Z.Geophys. Soc. Newsletter, 27, 20 - 28.

Suyehiro, K. (1988). Controlled source seismology, in:, The Ocean Basins and Margins, 7B, Nairn, A.E. M., Stehli, F.G. and Uyeda, S. eds., Plenum Press, New York .

Sverev, S. M. and Yaroshevskay, G. A. (1987). Deep seismic sounding on long range protiles in the Atlantic ocean,in:, Composition. Structure and Dynamics of the Lithosphere-Astenosphere System. Geodynamics Series, 16, A.G.U., Washington, U.S.A..

Uyeda, S. and Miyashiro, A. (1974). Plate tectonics and Japanese islands, Geol. Soc. Am. Bull., 85, 1159 1170.

VanderHilst, R., Engdahl, R., Spakmaun, W., and Nolet, G. (1991). Tomographic imaging of subducted lithosphere below northwest Pacitic island arcs, Nature, 353, 37 - 43.

Verna, R. K. (1960). The velocity of compressional waves in rocks to 10 kilobar, 1, J. Geophys. Res., 65, $1083-1102$.

Walcott, R. I. (1978). Present tectonics and Late Cenozoic evolution of New Zealand, Geophys. J. R. astr. Soc., 52, $137-164$.

Wilker, D. (1977). High-frequency $\mathrm{Pn}$ and Sn phases recorded in the Westem Pacific, J. Geophys. Res., 82. 335()$-3360$.

Watts, A. B., Weissel, J. K., Duncin, R. A., and Larson, R. L. (1988). Origin of the Louisville Ridge and its relationship to the Eltanin Fracture Zone system, J. Geophys. Res., 93, 3051 - 3077.

Weissel, J., Hayes, D., and Herron, E. M. (1977). Plate tectonics synthesis: the displacements between Australia, New Zealand and Antarctica since the Late Cretacesus, Mar. Geol., 25, 231 - 277.

Wenk, H. R., Bennett, K., Canova, G. R., and Molinari, A. (1991). Modelling plastic deformation of peridotite with the self-consistent theory, J. Geophys. Res., 96, 8337 - 8349.

Wenzel, F. and Sandmeier, K. J. (1988). Reflectivity method for dipping layers, J. Geophys. Res., 93. $15046-15056$.

Zhou, H. (1990). Mapping of P-wave slab anomalies heneath the Tonga, Kermadec and New Hebrides arcs, Phys. Eurth. Plan. Int., 61, 260 - 268. 


\section{Appendix A}

TableA. 1 The Weber Data Set

\begin{tabular}{|c|c|c|c|c|c|c|}
\hline $\begin{array}{l}\text { Event } \\
\text { I.D. }\end{array}$ & $\begin{array}{c}\text { Date } \\
\text { yymmdd }\end{array}$ & $\begin{array}{c}\text { Origin Time } \\
\text { b:m:s }\end{array}$ & $\begin{array}{c}\text { Latitude } \\
\mathrm{S}\end{array}$ & $\begin{array}{c}\text { Longitude } \\
\text { E } \\
\end{array}$ & $\begin{array}{l}\text { Depth } \\
(\mathrm{km})\end{array}$ & $\begin{array}{c}\text { Magnitude } \\
M_{L}\end{array}$ \\
\hline 01 & 900513 & $04: 23: 10.00$ & 40.34 & 176.27 & 19.0 & 6.3 \\
\hline 02 & 900513 & $04: 58: 59.67$ & 40.37 & 176.39 & 19.4 & 4.0 \\
\hline 03 & 900513 & $05: 02: 06.54$ & 40.36 & 176.40 & 30.0 & 4.0 \\
\hline 04 & 900513 & $05: 10: 51.91$ & 40.41 & 176.31 & 29.4 & 3.7 \\
\hline 05 & 900513 & $05: 22: 38.47$ & 40.41 & 176.34 & 24.1 & 3.6 \\
\hline 06 & 900513 & $05: 25: 16.30$ & 40.41 & 176.36 & 27.4 & 4.0 \\
\hline 07 & 900513 & $05: 34: 39.40$ & 40.37 & 176.36 & 20.6 & 3.5 \\
\hline 08 & 900513 & $05: 41: 34.13$ & 40.39 & 176.42 & 26.6 & 4.5 \\
\hline 09 & 900513 & $05: 50: 38.33$ & 40.43 & 176.47 & 31.0 & 3.5 \\
\hline 10 & 900513 & $05: 59: 13.09$ & 40.39 & 176.30 & 21.4 & 4.1 \\
\hline 11 & 900513 & $06: 08: 29.98$ & 40.52 & 176.32 & 17.7 & 3.7 \\
\hline 12 & 900513 & $06: 30: 30.80$ & 40.36 & 176.46 & 31.6 & 3.7 \\
\hline 13 & 900513 & $06: 37: 40.77$ & 40.37 & 176.41 & 34.1 & 3.9 \\
\hline 14 & 900513 & $06: 40: 31.08$ & 40.35 & 176.44 & 31.5 & 3.3 \\
\hline 25 & 900513 & $06: 55: 45.55$ & 40.40 & 176.52 & 31.0 & 3.5 \\
\hline 33 & 900513 & $06: 59: 33.84$ & 40.39 & 176.39 & 24.1 & 3.9 \\
\hline 26 & 900513 & $07: 11: 36.50$ & 40.42 & 176.45 & 30.4 & 4.4 \\
\hline 27 & 900513 & $07: 36: 36.54$ & 40.48 & 176.28 & 21.5 & 3.7 \\
\hline 34 & 900513 & 07:39:29.53 & 40.32 & 176.37 & 24.9 & 3.1 \\
\hline 28 & 900513 & 08:01:51.15 & 40.30 & 176.38 & 22.9 & 3.7 \\
\hline 29 & 900513 & $09: 16: 28.46$ & 40.40 & 176.52 & 30.4 & 4.2 \\
\hline 30 & 900513 & $09: 37: 05.17$ & 40.36 & 176.37 & 15.4 & 3.5 \\
\hline 15 & 900513 & $10: 10: 56.60$ & 40.45 & 176.49 & 29.3 & 4.9 \\
\hline 16 & 900513 & 13:00:32.94 & 40.42 & 176.37 & 34.2 & 4.7 \\
\hline 17 & 900513 & $15: 47: 49.20$ & 40.32 & 176.41 & 34.2 & 4.1 \\
\hline 18 & 900513 & $18: 32: 25.96$ & 40.40 & 176.35 & 27.5 & 4.2 \\
\hline 19 & 900513 & $19: 35: 14.45$ & 40.43 & 176.43 & 34.3 & 4.1 \\
\hline 20 & 900513 & $21: 58: 00.92$ & 40.28 & 176.00 & 19.8 & 4.1 \\
\hline 36 & 900514 & $01: 11: 55.07$ & 40.19 & 176.35 & 21.3 & 3.2 \\
\hline 37 & 900514 & $01: 57: 16.04$ & 40.42 & 176.17 & 14.8 & 4.0 \\
\hline 39 & 900514 & $04: 17: 14.21$ & 40.26 & 176.28 & 20.7 & 3.4 \\
\hline 40 & 900514 & $06: 03: 55.42$ & 40.31 & 176.20 & 18.4 & 2.9 \\
\hline 41 & 900514 & $06: 09: 22.94$ & 40.31 & 176.30 & 15.5 & 3.2 \\
\hline 43 & 900514 & $06: 33: 54.02$ & 40.31 & 176.26 & 19.3 & 3.2 \\
\hline 44 & 900514 & $07: 51: 32.93$ & 40.25 & 176.35 & 20.1 & 3.2 \\
\hline 36 & 900514 & $08: 02: 16.59$ & 40.23 & 176.33 & 20.7 & 3.5 \\
\hline 37 & 900514 & $12: 31: 11.91$ & 40.33 & 176.24 & 19.2 & 2.9 \\
\hline 38 & 900514 & $12: 37: 51.81$ & 40.34 & 176.33 & 13.1 & 4.2 \\
\hline 39 & 900514 & $14: 11: 39.27$ & 40.28 & 176.31 & 21.8 & 3.5 \\
\hline 40 & 900514 & $16: 14: 05.93$ & 40.26 & 176.34 & 26.5 & 2.9 \\
\hline 41 & 900514 & $18: 58: 13.84$ & 40.19 & 176.34 & 15.8 & 2.5 \\
\hline 43 & 900515 & $04: 59: 11.92$ & 40.24 & 176.32 & 21.2 & 3.2 \\
\hline 44 & 900515 & $12: 39: 14.78$ & 40.32 & 176.39 & 19.2 & 4.0 \\
\hline 45 & 900515 & $19: 24: 02.63$ & 40.37 & 176.29 & 14.8 & 3.1 \\
\hline 21 & 900515 & $04: 21: 50.09$ & 40.34 & 176.29 & 17.9 & 4.3 \\
\hline 22 & 900516 & $14: 26: 41.38$ & 40.35 & 176.31 & 18.9 & 3.9 \\
\hline 49 & 900516 & $17: 20: 09.03$ & 40.24 & 176.27 & 22.3 & 3.2 \\
\hline 23 & 900517 & $00: 57: 38.68$ & 40.24 & 176.31 & 22.1 & 4.5 \\
\hline
\end{tabular}




\begin{tabular}{|ccccccc|}
\hline $\begin{array}{c}\text { Event } \\
\text { I.D. }\end{array}$ & $\begin{array}{c}\text { Date } \\
\text { yymmdd }\end{array}$ & $\begin{array}{c}\text { Origin Time } \\
\text { h:m:s }\end{array}$ & $\begin{array}{c}\text { Latitude } \\
\text { S }\end{array}$ & $\begin{array}{c}\text { Longitude } \\
\text { E }\end{array}$ & $\begin{array}{c}\text { Depth } \\
\text { (km) }\end{array}$ & $\begin{array}{c}\text { Magnitude } \\
M_{L}\end{array}$ \\
\hline 50 & 900517 & $01: 02: 41.58$ & 40.22 & 176.31 & 21.5 & 3.3 \\
52 & 900517 & $05: 01: 23.90$ & 40.22 & 176.33 & 22.5 & 3.5 \\
53 & 900517 & $09: 54: 42.99$ & 40.28 & 176.28 & 21.1 & 3.8 \\
54 & 900517 & $17: 09: 52.16$ & 40.28 & 176.38 & 21.8 & 3.5 \\
55 & 900518 & $00: 43: 11.97$ & 40.38 & 176.31 & 20.0 & 3.5 \\
35 & 900519 & $22: 57: 31.68$ & 40.47 & 176.34 & 33.4 & 4.5 \\
\hline
\end{tabular}

TableA. 2 Some other Central North Island Events recorded on the L-Network

\begin{tabular}{|c|c|c|c|c|c|c|}
\hline $\begin{array}{l}\text { Event } \\
\text { I.D. }\end{array}$ & $\begin{array}{c}\text { Date } \\
\text { yymmdd }\end{array}$ & $\begin{array}{c}\text { Origin Time } \\
\text { h:m:s }\end{array}$ & $\begin{array}{c}\text { Latitude } \\
\mathrm{S}\end{array}$ & $\begin{array}{c}\text { Longitude } \\
\text { E }\end{array}$ & $\begin{array}{l}\text { Depth } \\
(\mathbf{k m})\end{array}$ & $\begin{array}{c}\text { Magnitude } \\
M_{L}\end{array}$ \\
\hline 106 & 900309 & $16: 53: 40.04$ & 40.52 & 176.27 & 38.1 & 3.0 \\
\hline 107 & 900310 & $02: 20: 26.70$ & 40.47 & 176.37 & 41.2 & 3.3 \\
\hline 108 & 900311 & $00: 42: 19.40$ & 39.93 & 177.05 & 32.3 & 3.6 \\
\hline 132 & 900313 & $09: 45: 35.06$ & 40.89 & 175.59 & 44.8 & 3.1 \\
\hline 109 & 900318 & $13: 35: 09.89$ & 40.38 & 176.42 & 40.5 & 3.0 \\
\hline 133 & 900318 & $16: 00: 31.55$ & 41.17 & 175.82 & 26.3 & 2.2 \\
\hline 110. & 900318 & $09: 49: 44.18$ & 40.35 & 176.45 & 39.0 & 2.9 \\
\hline 111 & 900319 & $09: 47: 04.24$ & 40.46 & 176.39 & 40.9 & 3.9 \\
\hline 134 & 900321 & $12: 58: 53.58$ & 41.04 & 175.26 & 23.4 & 2.0 \\
\hline 112 & 900322 & $17: 33: 04.38$ & 40.43 & 176.47 & 40.2 & 3.4 \\
\hline 113 & 900323 & $04: 06: 20.81$ & 40.49 & 175.97 & 22.6 & 3.6 \\
\hline 114 & 900323 & $07: 17: 21.31$ & 39.86 & 176.65 & 61.5 & 3.8 \\
\hline 115 & 900326 & $15: 41: 43.53$ & 40.44 & 176.51 & 40.5 & 3.2 \\
\hline 116 & 900330 & $06: 07: 33.92$ & 40.39 & 176.41 & 41.6 & 4.0 \\
\hline 135 & 900330 & $15: 25: 20.42$ & 40.41 & 176.81 & 31.2 & 3.8 \\
\hline 117 & 900331 & $03: 36: 22.50$ & 40.65 & 176.55 & 28.0 & 3.2 \\
\hline 118 & 900406 & $20: 36: 00.35$ & 40.41 & 176.42 & 41.4 & 3.6 \\
\hline 119 & 900407 & $19: 22: 06.82$ & 40.40 & 176.47 & 39.5 & 3.3 \\
\hline 136 & 900408 & $20: 56: 08.77$ & 40.12 & 176.17 & 66.5 & 4.8 \\
\hline 120 & 900408 & $21: 36: 01.19$ & 40.64 & 175.88 & 33.3 & 4.0 \\
\hline 121 & 900408 & $21: 38: 27.67$ & 40.64 & 175.88 & 33.3 & 3.4 \\
\hline 122 & 900408 & $21: 53: 13.45$ & 40.40 & 176.44 & 39.8 & 3.0 \\
\hline 131 & 900410 & $17: 46: 52.98$ & 40.72 & 175.87 & 32.2 & 4.5 \\
\hline 123 & 900417 & $07: 09: 31.30$ & 40.63 & 175.53 & 31.7 & 2.6 \\
\hline 137 & 900421 & $13: 13: 33.67$ & 40.36 & 176.60 & 23.6 & 3.5 \\
\hline 124 & 900422 & $09: 55: 15.82$ & 40.44 & 176.48 & 40.5 & 3.2 \\
\hline 125 & 900428 & $04: 21: 39.80$ & 40.44 & 176.48 & 40.5 & 3.2 \\
\hline 126 & 900429 & $06: 24: 22.36$ & 40.33 & 176.30 & 23.6 & 3.5 \\
\hline 127 & 900430 & $23: 26: 50.51$ & 40.42 & 176.43 & 42.2 & 3.6 \\
\hline 128 & 900502 & $02: 14: 17.52$ & 40.40 & 176.38 & 33.0 & 2.6 \\
\hline 138 & 900505 & $11: 34: 35.08$ & 40.49 & 176.92 & 41.9 & 3.8 \\
\hline 129 & 900506 & $11: 46: 40.70$ & 40.13 & 176.71 & 61.7 & 3.7 \\
\hline 130 & 900512 & $03: 32: 29.42$ & 40.40 & 176.38 & 29.9 & 4.3 \\
\hline
\end{tabular}


TableA. 3 L. Tennyson Events recorded on the L-Network

\begin{tabular}{|ccccccc|}
\hline $\begin{array}{c}\text { Event } \\
\text { I.D. }\end{array}$ & $\begin{array}{c}\text { Date } \\
\text { yymmdd }\end{array}$ & $\begin{array}{c}\text { Origin Time } \\
\text { b:m:s }\end{array}$ & $\begin{array}{c}\text { Latitude } \\
\text { S }\end{array}$ & $\begin{array}{c}\text { Longitude } \\
\text { E }\end{array}$ & $\begin{array}{c}\text { Depth } \\
\text { (km) }\end{array}$ & $\begin{array}{c}\text { Magnitude } \\
M_{L}\end{array}$ \\
\hline 307 & 900310 & $01: 03: 21.11$ & 42.26 & 172.72 & 17.4 & 3.3 \\
301 & 900310 & $12: 09: 28.74$ & 42.24 & 172.73 & 5.0 & 4.2 \\
303 & 900311 & $13: 05: 00.13$ & 42.24 & 172.73 & 5.0 & 4.2 \\
304 & 900329 & $10: 43: 57.91$ & 42.30 & 172.78 & 5.0 & 3.6 \\
315 & 900329 & $11: 13: 28.77$ & 42.26 & 172.69 & 12.0 & 3.7 \\
318 & 900410 & $00: 32: 41.82$ & 42.27 & 172.70 & 5.0 & 2.8 \\
319 & 900412 & $11: 20: 50.76$ & 42.23 & 172.71 & 11.1 & 3.3 \\
320 & 900412 & $18: 28: 11.87$ & 42.25 & 172.69 & 5.0 & 3.2 \\
321 & 900412 & $19: 10: 25.11$ & 42.24 & 172.70 & 5.0 & 3.4 \\
323 & 900415 & $20: 36: 50.30$ & 42.24 & 172.74 & 5.0 & 3.2 \\
328 & 900425 & $14: 58: 57.90$ & 42.28 & 172.69 & 5.0 & 3.6 \\
329 & 900427 & $07: 03: 10.76$ & 42.27 & 172.67 & 5.0 & 3.2 \\
332 & 900429 & $09: 53: 52.94$ & 42.28 & 172.72 & 5.0 & 3.1 \\
334 & 900506 & $00: 40: 29.72$ & 42.28 & 172.70 & 5.0 & 3.4 \\
335 & 900507 & $02: 10: 59.84$ & 42.29 & 172.69 & 2.8 & 3.1 \\
336 & 900510 & $06: 30: 52.64$ & 42.30 & 172.74 & 0.8 & 3.2 \\
\hline
\end{tabular}


Appendix B

MULTIPLE FILTER ANALYSIS

Fourier Transform Signal $f(t) \rightarrow F(\omega)$

Choose Centre Frequencies

$\omega_{1}, \ldots, \omega_{j}, \ldots, \omega_{n}$

Construct Gaussian Band-Pass Filter

$G_{j}(\omega)= \begin{cases}0 & \omega<\omega_{j}^{l} \\ e^{-\alpha\left(\frac{\left.\omega-\omega_{j}\right)^{2}}{\omega_{j}}\right.} & \omega_{j}^{l} \leq \omega \leq \omega_{j}^{u} \\ 0 & \omega>\omega_{j}^{u}\end{cases}$

where $\omega_{j}^{l}=(1-B A N D) \omega_{j}$ $\omega_{j}^{u}=(1+B A N D) \omega_{j}$

Multiply Spectrum in turn by

$G_{1}(\omega), \ldots, G_{j}(\omega), \ldots, G_{n}(\omega)$

$F(\omega) . G_{j}(\omega)->H_{j}(\omega)$

t.

Construct Quadrature Spectrum

$Q_{j}(\omega)=i H_{j}(\omega)$

FFT $H_{j}(\omega)$ and $Q_{j}(\omega)$ back to time

$H_{j}(\omega) \rightarrow h_{j}(t) ; Q_{j}(\omega) \rightarrow q_{j}(t)$

Envelope Function:

$h_{j}(t)-i q_{j}(t)$

Instantaneous Amplitude:

$A_{j}(t)=\left|h_{j}(t)-i q_{j}(t)\right|$

Output result as a matrix of numbers.

Rows represent time-sampled envelope of the signal, band-pass filtered at the given frequency. 
172 


\section{Appendix C}

\section{C1 Take-off angle within plane dipping slab}

In this section, the expression for $\alpha$, the take-off angle of a refracted ray down a plane dipping slab, as in Section 3.1, is derived.

In Figure 3.1, consider the origin of the $x, y, z$ coordinate system to be temporarily shifted, for simplicity, to point $\mathrm{O}$, the origin. $\mathrm{E}$ is the epicentre. The equation of the dipping plane $\mathrm{ABCD}$ is

$$
x \tan \delta+z=0
$$

Let the equation of the plane of propagation ORLN be

$$
A x+B y+C z+D=0
$$

This plane must contain the unit vector along the normal ON, given by

$$
\mathbf{n}=(\sin \delta, 0, \cos \delta)
$$

and the unit vector along $O R$, given by

$$
\mathrm{OR}=(\sin \alpha \cos \delta, \cos \alpha,-\sin \alpha \sin \delta)
$$

The plane ORLN also contains the point $(0,0,0)$. Substituting these three vectors into equation $\mathrm{C} 1$ we get

$$
x-\frac{\tan \alpha}{\cos \delta} y-z \tan \delta=0
$$

for the equation of plane ORLN.

The equation of the line where this plane intersects the surface is given by putting $z=h$. Thus this line has the equation

$$
x-\frac{\tan \alpha}{\cos \delta} y=h \tan \delta
$$

The plane ORLN must also contain the receiver point $\mathrm{L}$, whose coordinates are $(X, Y, H)$. Therefore

$$
\begin{gathered}
X-\frac{\tan \alpha}{\cos \delta} Y-h \tan \delta=0 \\
\tan \alpha=\cos \delta\left(\frac{X-h \tan \delta}{Y}\right)
\end{gathered}
$$

The term $h \tan \delta$ is equal to the distance NE in Fig.3.1, so that if we choose to work in the 
coordinate system whose origin is at $\mathrm{N}$, as in Chapter 3, then the angle $\alpha$ may be written as

$$
\tan \alpha=\frac{X}{Y} \cos \delta
$$

In the case of an updip travelling wave, the derivation is analogous, and we get

$$
\tan \alpha=\left(\frac{X+h \tan \delta}{Y}\right) \cos \delta
$$

so that, if the origin of the coordinate system is again taken to be at the point where the normal to the dipping slab at the source hits the surface, then we still have

$$
\tan \alpha=\frac{X}{Y} \cos \delta
$$

C2 Travel time of the ray (derivation due to Ansell (pers. comm., 1990))

Consider first the plane of propagation (Figure $\mathrm{C} 1$ ). ORL is the ray. $\gamma$ is the critical angle, given by

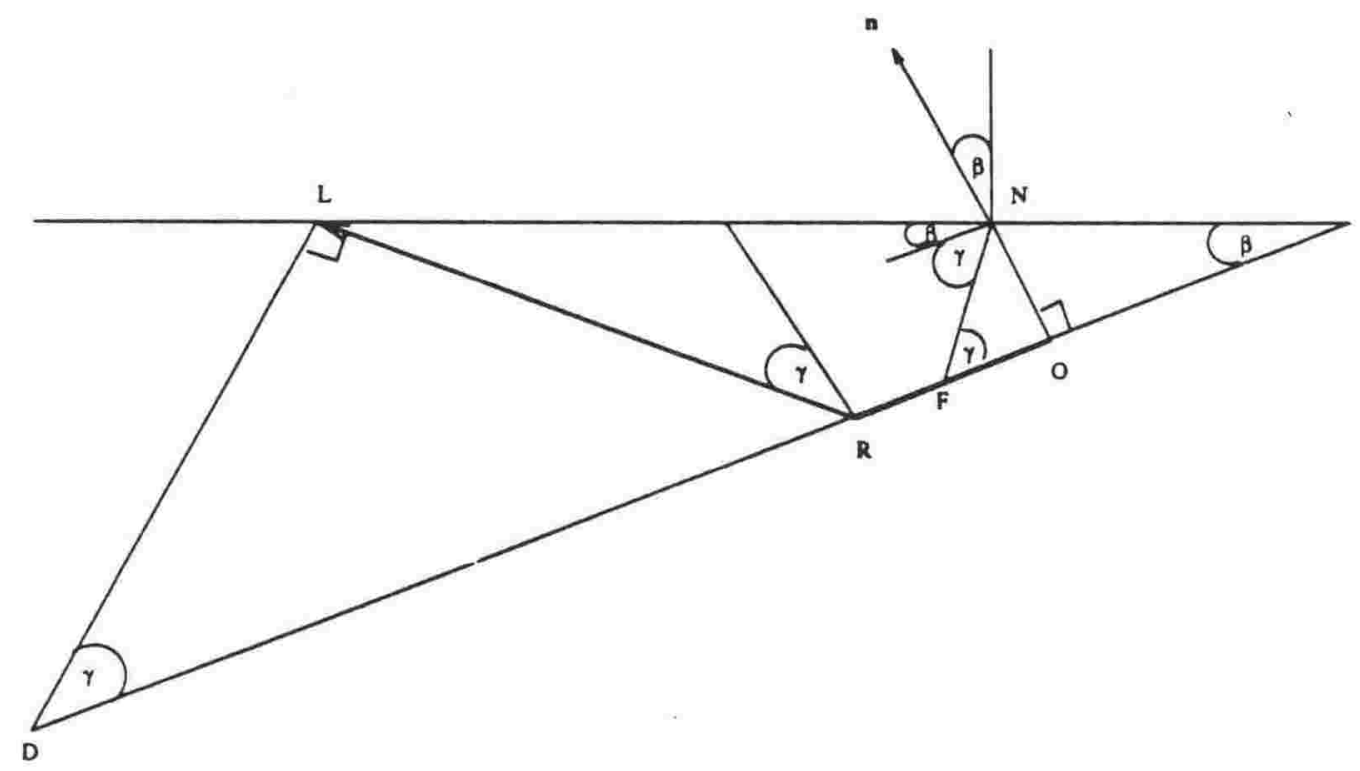

Fig. C1 Plane of propagation of refracted ray $O R L$ 


$$
\sin \gamma=\frac{v_{1}}{v_{2}}
$$

The travel time $\mathrm{T}$ is given by

$$
\begin{gathered}
T=\frac{O R}{v_{2}}+\frac{R L}{v_{1}} \\
T=\frac{O R}{v_{2}}+\frac{R D \sin \gamma}{v_{1}} \\
T=\frac{O R}{v_{2}}+\frac{R D}{v_{2}} \\
T=\frac{O D}{v_{2}}=\frac{O F+F D}{v_{2}}
\end{gathered}
$$

In the parallelogram NLDF, we have

$$
\frac{N L}{\sin \gamma}=\frac{F D}{\sin (\gamma+\beta)}
$$

Therefore

$$
\begin{gathered}
T=\frac{O F+N L \sin (\gamma+\beta) / \sin \gamma}{v_{2}} \\
T=\frac{O N \cot \gamma+N L \sin (\gamma+\beta) / \sin \gamma}{v_{2}} \\
T=\frac{d \cot \gamma}{v_{2}}+\frac{N L \sin (\gamma+\beta)}{v_{2} \sin \gamma}
\end{gathered}
$$

where $\mathrm{ON}=\mathrm{d}$

or:

$$
T=\frac{d \cot \gamma}{v_{2}}+\frac{N L}{v_{2}}(\cos \beta+\cot \gamma \sin \beta)
$$

We also have

$$
\text { NL. } \mathbf{n}=\sin \beta
$$

where $\mathrm{NL}$ is the unit vector along NL.

Now consider the plane in its 3-dimensional setting (Figure 3.1) with the origin of the coordinate system again at $\mathrm{N}$. (X, Y) are the coordinates measured from N.

$$
\mathrm{NL}=\left(\frac{X}{\sqrt{X^{2}+Y^{2}}}, \frac{Y}{\sqrt{X^{2}+Y^{2}}}, 0\right)
$$




$$
\text { NL. } \mathbf{n}=\left(\frac{X}{\sqrt{X^{2}+Y^{2}}}, \frac{Y}{\sqrt{X^{2}+Y^{2}}}, 0\right),(\sin \delta, 0, \cos \delta)
$$

Therefore, using equation $\mathrm{C} 4$

$$
\begin{gathered}
\sin \beta=\frac{X}{\sqrt{X^{2}+Y^{2}}} \sin \delta \\
\cos \beta=\sqrt{1-\sin ^{2} \beta}=\sqrt{1-\frac{X^{2} \sin ^{2} \delta}{X^{2}+Y^{2}}}=\sqrt{\frac{X^{2}+Y^{2}-X^{2} \sin ^{2} \delta}{X^{2}+Y^{2}}} \\
\cos \beta=\sqrt{\frac{X^{2} \cos ^{2} \delta+Y^{2}}{X^{2}+Y^{2}}}
\end{gathered}
$$

Therefore equation $\mathrm{C} 3$ becomes

$$
\begin{gathered}
T=\frac{d \cot \gamma}{v_{2}}+\frac{\sqrt{X^{2}+Y^{2}}}{\nu_{2}}\left(\sqrt{\frac{X^{2} \cos ^{2} \delta+Y^{2}}{X^{2}+Y^{2}}}+\frac{X \cot \gamma \sin \delta}{\sqrt{X^{2}+Y^{2}}}\right) \\
T=\frac{d \cot \gamma}{v_{2}}+\frac{1}{v_{2}}\left(\sqrt{X^{2} \cos ^{2} \delta+Y^{2}}+X \cot \gamma \sin \delta\right)
\end{gathered}
$$

To find the surface wavefront, we must find the surface locus of constant $T, i . e$.

$$
T(x, y)=C(\text { constant })
$$

Thus

$$
\begin{gathered}
\frac{d \cot \gamma}{v_{2}}+\frac{1}{v_{2}}\left(\sqrt{X^{2} \cos ^{2} \delta+Y^{2}}+X \cot \gamma \sin \delta\right)=C \\
v_{2}\left(C-\frac{d \cot \gamma}{v_{2}}\right)=\sqrt{X^{2} \cos ^{2} \delta+Y^{2}}+X \cot \gamma \sin \delta \\
\text { Let } D=v_{2}\left(C-\frac{d \cot \gamma}{v_{2}}\right)=\text { constant }
\end{gathered}
$$

Then

$$
\begin{gathered}
D-X \cot \gamma \sin \delta=\sqrt{X^{2} \cos ^{2} \delta+Y^{2}} \\
D^{2}=X^{2}\left(\cos ^{2} \delta-\cot ^{2} \gamma \sin ^{2} \delta\right)+2 D X \cot \gamma \sin \delta+Y^{2} \\
\frac{D^{2}}{\cos ^{2} \delta-\cot ^{2} \gamma \sin ^{2} \delta}=X^{2}+\frac{2 D \cot \gamma \sin \delta}{\cos ^{2} \delta-\cot ^{2} \gamma \sin ^{2} \delta} X+\frac{Y^{2}}{\cos ^{2} \delta-\cot ^{2} \gamma \sin ^{2} \delta}
\end{gathered}
$$




$$
\frac{D^{2}}{\cos ^{2} \delta-\cot ^{2} \gamma \sin ^{2} \delta}=\left(X+\frac{D \cot \gamma \sin \delta}{\cos ^{2} \delta-\cot ^{2} \gamma \sin ^{2} \delta}\right)^{2}-\frac{D^{2} \cot ^{2} \gamma \sin ^{2} \delta}{\cos ^{2} \delta-\cot ^{2} \gamma \sin ^{2} \delta^{2}}+\frac{Y^{2}}{\cos ^{2} \delta-\cot ^{2} \gamma \sin ^{2} \delta}
$$

Multiplying by $\cos ^{2} \delta-\cot ^{2} \gamma \sin ^{2} \delta$ :

$$
\begin{gathered}
D^{2}=\left(\cos ^{2} \delta-\cot ^{2} \gamma \sin ^{2} \delta\right)(X+E)^{2}-\frac{D^{2} \cot ^{2} \gamma \sin ^{2} \delta}{\cos ^{2} \delta-\cot ^{2} \gamma \sin ^{2} \delta}+Y^{2} \\
D^{2}\left(1+\frac{\cot ^{2} \gamma \sin ^{2} \delta}{\cos ^{2} \delta-\cot ^{2} \gamma \sin ^{2} \delta}\right)=\left(\cos ^{2} \delta-\cot ^{2} \gamma \sin ^{2} \delta\right)(X+E)^{2}+Y^{2}
\end{gathered}
$$

or:

$$
\left(\cos ^{2} \delta-\cot ^{2} \gamma \sin ^{2} \delta\right)(X+E)^{2}+Y^{2}=\frac{D^{2} \cos ^{2} \delta}{\cos ^{2} \delta-\cot ^{2} \gamma \sin ^{2} \delta}
$$

where $E=\frac{D \cot \gamma \sin \delta}{\cos ^{2} \delta-\cot ^{2} \gamma \sin ^{2} \delta}$

The above is the equation of an ellipse with centre (-E, 0). Note that if $\delta=0$ (i.e. a horizontal slab), $\mathrm{E}$ becomes 0 and the equation becomes that of a circle.

\section{C4. Alternative derivation of surface slowness}

The horizontal slowness $\mathbf{p}$ at the surface is given by

$$
\mathrm{p}=\nabla T(X, Y)=\left(\frac{\partial T}{\partial X}, \frac{\partial T}{\partial Y}\right)
$$

Using the equation for $\mathrm{T}$ we get

$$
\mathbf{p}=\frac{1}{v_{2}}\left(\frac{X \cos ^{2} \delta}{\sqrt{X^{2} \cos ^{2} \delta+Y^{2}}}+\cot \gamma \sin \delta, \frac{Y}{\sqrt{X^{2} \cos ^{2} \delta+Y^{2}}}\right)
$$

Using the relation

$$
\tan \alpha=\frac{X \cos \delta}{Y}
$$

obtained in Section C.1, we find 


$$
\begin{gathered}
\mathbf{p}=\left(\frac{\cos ^{2} \delta}{\sqrt{\cos ^{2} \delta+\frac{\cos ^{2} \delta}{\tan ^{2} \alpha}}}+\cot \gamma \sin \delta, \frac{1}{\sqrt{\tan ^{2} \alpha+1}}\right) \\
\mathbf{p}=(\cos \delta \sin \alpha+\cot \gamma \sin \delta, \cos \alpha)
\end{gathered}
$$

This is identical to equation 3.2 . 


\section{Appendix D}

\section{The EARSS Instrument Response}

The frequency response of the EARSS seismograph can be written as the product of the frequency response functions of the seismometer, the coil and the electronic components as follows (Chadwick, 1991):

$$
g(\omega)=\omega r_{1}(\omega) r_{2}(\omega) s(\omega)
$$

where

$\omega$ is the angular frequency in radians;

$g(\omega)$ is the total instrument response;

$r_{1}(\omega)$ is the response of the anti-alias, second-order Butterworth low-pass filter in the EARSS recorder, given by

$$
r_{1}(\omega)=\frac{1}{\frac{1}{\omega}-i}
$$

$r_{2}(\omega)$ is the response of the first-order Butterworth high-pass filter, with half-power at $(1 / 2 \pi) \mathrm{Hz}$, and corner frequency $\omega_{B}$ given by $0.6 f_{N}, f_{N}$ being the Nyquist frequency $(50 \mathrm{~Hz}), r_{2}(\omega)$ is given by

$$
r_{2}=\frac{1}{\left(\frac{\omega}{\omega_{B}}\right)^{2}+\sqrt{2} i \frac{\omega}{\omega_{B}}-1}
$$

and $s(\omega)$ is the seismometer response, given by

$$
s(\omega)=\frac{U \omega^{2}}{\omega^{2}+2 i h \omega \omega_{c}-\omega_{c}^{2}}
$$

in which $U, h$ and $\omega_{c}$ are the gain constant, damping constant and critical angular frequency of the seismometer respectively. Chadwick(1991) measured these constants by a least-squares inversion technique which fits the observed response curve to the theoretical one. Typical values for an EARSS seismograph operating at $100 \mathrm{~Hz}$ sampling frequency are:

$$
\begin{gathered}
U=1.563 \times 10^{10} \\
h=0.587 \\
\omega_{c}=6.58 \text { radians }
\end{gathered}
$$

The factor $\omega$ in equation D1 in effect accounts for the response of the seismometer coil. 


\section{Addendum to Chapters 2/3}

(i) Error in the apparent velocity arising from possible short-wavelength heterogeneities in the medium between refractor and seismograph network

In using the arrival times of the $P_{n}$ phase at stations of the L-network to invert for wavefront speed and azimuth, it has been assumed that the structure between the refractor (along which $P_{n}$ travels) and the L-network is homogeneous, except for surface topography, corrections for which were made to the arrival times at the different stations (Section 2.4). Although the linear dimensions of the L-network are small (approximately $3 \mathrm{~km}$ ), it is possible that there are shortwavelength heterogeneities, especially in the overlying crust, which represents a tectonically deformed region on the Wellington peninsula. Such heterogeneities would introduce differences in the time-terms at the receiver end for the same phase $\left(\mathrm{P}_{\mathrm{n}}\right)$ arriving at different stations of the network. Corrections for these differences are of the same nature as those for station elevations. An evaluation of the uncertainty in the apparent velocity introduced by these time-term differences is here attempted.

Consider, in the simplified case of a plane, horizontal refractor, a wavefront incident on two stations of the network, as shown:

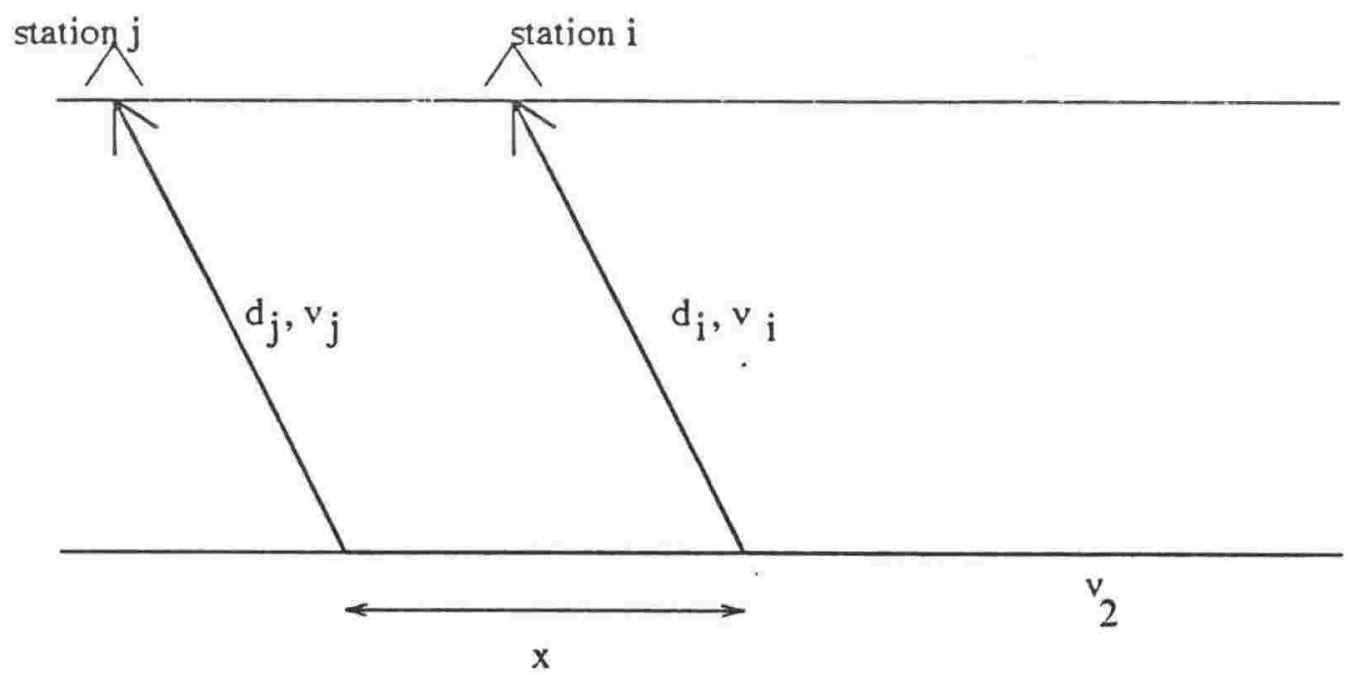

Let $d_{i}, v_{i}$ be the distance and average P-velocity above the refractor applicable to the wave reaching station $i$. If $d_{i}, v_{i}$ are the same for all stations, then the arrival time difference $t_{j}-t_{i}$ yields the velocity $v_{2}$ by $v_{2}=x\left(t_{j}-t_{i}\right)$. But if they are different, this introduces a time-term difference of

$$
\Delta\left(t_{j}-t_{i}\right)=\frac{d_{j}}{v_{j}}-\frac{d_{i}}{v_{i}}
$$

or, if it is assumed that the distances $d_{i}, d_{j}$ are equal, then 


$$
\Delta\left(t_{j}-t_{i}\right)=d\left(\frac{1}{v_{j}}-\frac{1}{v_{i}}\right)
$$

From Figure 5.5 (basic velocity model used in waveform matching), the average crustal Pvelocity is $5.68 \mathrm{~km} / \mathrm{s}$, and the average velocity in the subducted crust and upper mantle above the refractor is $7.30 \mathrm{~km} / \mathrm{s}$. A difference of $1 \%$ in the crustal velocity (where the main small-scale heterogeneity is expected to occur) will yield a change in the average velocity above the refractor of $\pm 0.02 \mathrm{~km} / \mathrm{s}$ in $6.49 \mathrm{~km} / \mathrm{s}$. This produces a time-term difference of the order of $\pm 0.02 \mathrm{~s}$.

Since the apparent velocity was measured by a least-squares inversion of the arrival times at 6 network stations, it was decided to estimate the effect of the above uncertainty by adding timeterm differences arbitrarily to the observed arrival times, and re-doing the inversion several times to observe the variations in $c$ and $\phi$. This gave a measure of the stability of the inversion with respect to such fluctuations. The arrival times from event number 01 were chosen as a test data set. This event yielded $c=8.53 \mathrm{~km} / \mathrm{s}$ and $\phi=53.7^{\circ}$.

Clearly, the effect on the best-fit result is sensitive to which stations are involved. As expected, the maximum variation in $c$ was observed when adding (or subtracting) time-term differences to the end-stations PDD and NDE.

Adding a time-term correction of $+1-0.02 \mathrm{~s}$ to single stations SAL, RKK, KSE and SEF respectively caused the solution for $c$ to swing between $8.40 \mathrm{~km} / \mathrm{s}$ and $8.61 \mathrm{~km} / \mathrm{s}$, an approximate error of $\pm 0.1 \mathrm{~km} / \mathrm{s}$.

Adding a time-term correction of $\pm 0.02 \mathrm{~s}$ to stations PDD and NDE respectively caused the solution for $c$ to swing between $8.73 \mathrm{~km} / \mathrm{s}$ and $8.42 \mathrm{~km} / \mathrm{s}$, an uncertainty of $\pm 0.2 \mathrm{~km} / \mathrm{s}$. This is approximately equivalent to having a $1 \%$ difference in crustal P-velocity between the ends of the array - a distance of around $4.3 \mathrm{~km}$.

Adding random time-term corrections to all stations in the array produced variations in $c$ within $\pm 0.2 \mathrm{~km} / \mathrm{s}$.

The extreme situation af adding a correction of $0.02 \mathrm{~s}$ to PDD and $-0.02 \mathrm{~s}$ to NDE, and vice - versa (representing an approximately $2 \%$ change in the crustal velocity) caused variations in $c$ of $\pm 0.4 \mathrm{~km} / \mathrm{s}$.

In all the above cases, the swing in the slowness azimuth, $\phi$, was not greater than $\pm 3^{\circ}$.

In conclusion, the apparent velocity obtained by inverting the array arrival times is considerably sensitive to lateral heterogeneity in the crust. In the absence of a proper leastsquares analysis to estimate the magnitude of the time-term corrections at the 6 stations, the assessment of the error in the wavefront speed due to lateral heterogeneity would be that a $1 \%$ change in the crustal P-velocity between the ends of the seismic network produces an uncertainty of $\pm 0.2 \mathrm{~km} / \mathrm{s}$ in $c$ and $\pm 3^{\circ}$ in $\phi$. Added to the observational errors (due primarily to uncertainty in pick times) of $\pm 0.2 \mathrm{~km} / \mathrm{s}$, this means that the apparent velocity should be quoted as $8.70 \pm 0.4$ $\mathrm{km} / \mathrm{s}$. This is a fairly large uncertainty, however it is important to note that the presence of a high- 
velocity layer (exceeding $8.6 \mathrm{~km} / \mathrm{s}$ ) within the subducted Pacific plate is revealed through other means, such as travel time curves (Section 2.4), waveform matching of the first few seconds of the signal (Chapter 5), which could not be achieved with a normal upper mantle structure, and the Lake Tennyson data set (Section 2.5).

\section{(ii) Final error in the quoted true velocity}

As explained in Chapter 3, when converting from the apparent velocity to the true P-velocity below the refractor, it was found that an analytical method could not be used, since the form of curvature of the slab appeared to influence quite highly the apparent velocity. Of necessity, therefore, a method of forward modelling (3-d ray tracing) had to be applied. This involved uncertainties of a completely different nature to the ones involved in estimating the apparent velocity from the observations. These uncertainties arose mainly because of the non-unique nature of the geometry/velocity maodel, and trade-offs between certain features of the model. The forward modelling may be thought of more suitably as an exercise in demonstrating, as clearly as possible, that the influence of the curvature of the slab is such that a single "conversion" from apparent to true P-velocity is not strictly justified, and that such conversions which assume a plane subducted slab in this region would yield misleadingly high results for the true velocity. Nevertheless, it is possible to state resonable bounds for the P-velocity below the refractor, as follows:

The uncertainty in the modelling arises mainly because (a) the precise nature of the slab surface curvature is not known, and (b) the P-velocity in the layer immediatel overlying the subducted slab surface, which has a significant effect on the apparent velocity, cannot be ascertained by deductive means or form results of other studies. From the modelling outlined in Section 3.4 (see particularly Figures 3.7 and 3.9), if we discard the model of a regular cylindrical curvature (Ansell and Bannister, 1991), a given apparent velocity could be modelled by a true velocity that is between 0.05 and $0.15 \mathrm{~km} / \mathrm{s}$ higher. This value is obtained promarily from the effect of the P-velocity directly above the slab surface, but also includes the effect of small departures of the slab curvature from that of Figure 3.8/Table 3.3. It means that an apparent velocity of $8.70 \mathrm{~km} / \mathrm{s}$ would correspond to a true velocity of $8.80 \pm 0.05 \mathrm{~km} / \mathrm{s}$.

Since the total error in the apparent velocity may be as large as $\pm 0.4 \mathrm{~km} / \mathrm{s}$, then the true velocity should really be quoted with an uncertainty of $\pm 0.45 \mathrm{~km} / \mathrm{s}$. 


\section{Errata}

p19 last para, 13: $\quad 44.40^{\circ} \mathrm{S}$ to read $40.40^{\circ} \mathrm{S}$

p28 para 1 to continue: $\quad$...first portion, at station NDE is again notable.

p37 12 of Section 2.5.1: $\quad 8.7 \mathrm{~m} / \mathrm{s}$ to read $8.7 \mathrm{~km} / \mathrm{s}$

p53 Section 3.2.2: The slowness vector to read:

$$
\mathbf{s}=\frac{1}{v_{1}}\left(\begin{array}{c}
-\cos \delta \sin \gamma \sin \alpha+\sin \delta \cos \gamma \\
-\sin \gamma \cos \alpha \\
\sin \delta \sin \gamma \sin \alpha+\cos \delta \cos \gamma
\end{array}\right)
$$

p54 The equation for $c$ to read

$$
c=v_{2}\left(\cos ^{2} \alpha+\cot ^{2} \gamma \sin ^{2} \delta-\sin 2 \delta \sin \alpha \cot \gamma+\cos ^{2} \delta \sin ^{2} \alpha\right)^{-\frac{1}{2}}
$$

pl11 114: $\quad$ "P - SV" to read "SV - P"

116/17: $\quad$ "SV to P" to read "P to SV"

p161 Reference to Boudier, F. and Nicholas, A. : 1986 to read 1985

p173 1-3: $\quad(\mathrm{X}, \mathrm{Y}, \mathrm{H})$ to read $(\mathrm{X}, \mathrm{Y}, \mathrm{h})$ 


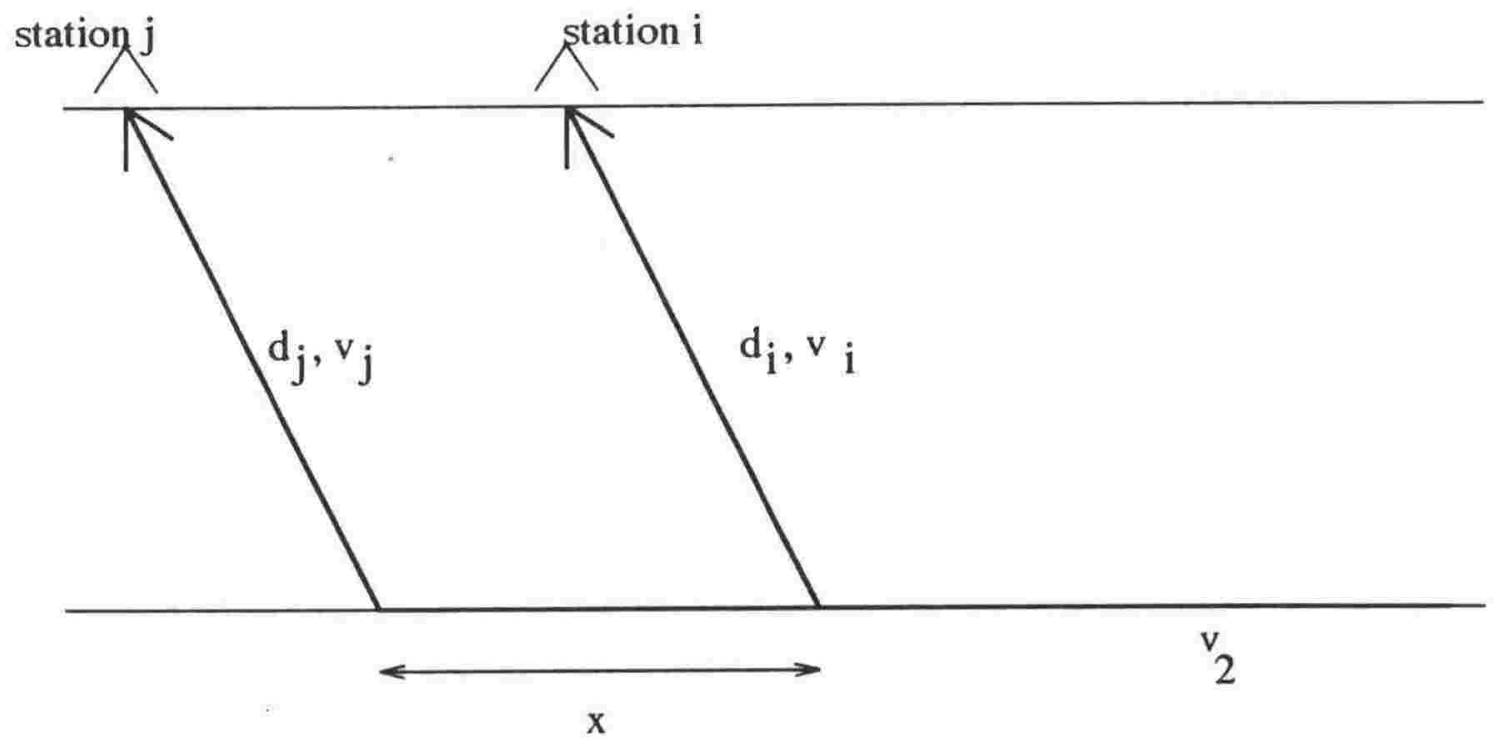

